\title{
Neuromuscular electrical stimulation in dyspneic COPD patients: a new training modality
}

Citation for published version (APA):

Sillen, M. J. H. (2014). Neuromuscular electrical stimulation in dyspneic COPD patients: a new training modality. [Doctoral Thesis, Maastricht University]. Datawyse / Universitaire Pers Maastricht. https://doi.org/10.26481/dis.20140410ms

Document status and date:

Published: 01/01/2014

DOI:

10.26481/dis.20140410ms

Document Version:

Publisher's PDF, also known as Version of record

\section{Please check the document version of this publication:}

- A submitted manuscript is the version of the article upon submission and before peer-review. There can be important differences between the submitted version and the official published version of record.

People interested in the research are advised to contact the author for the final version of the publication, or visit the DOI to the publisher's website.

- The final author version and the galley proof are versions of the publication after peer review.

- The final published version features the final layout of the paper including the volume, issue and page numbers.

Link to publication

\footnotetext{
General rights rights.

- You may freely distribute the URL identifying the publication in the public portal. please follow below link for the End User Agreement:

www.umlib.nl/taverne-license

Take down policy

If you believe that this document breaches copyright please contact us at:

repository@maastrichtuniversity.nl

providing details and we will investigate your claim.
}

Copyright and moral rights for the publications made accessible in the public portal are retained by the authors and/or other copyright owners and it is a condition of accessing publications that users recognise and abide by the legal requirements associated with these

- Users may download and print one copy of any publication from the public portal for the purpose of private study or research.

- You may not further distribute the material or use it for any profit-making activity or commercial gain

If the publication is distributed under the terms of Article $25 \mathrm{fa}$ of the Dutch Copyright Act, indicated by the "Taverne" license above, 
Neuromuscular electrical stimulation in dyspneic COPD patients: a new training modality

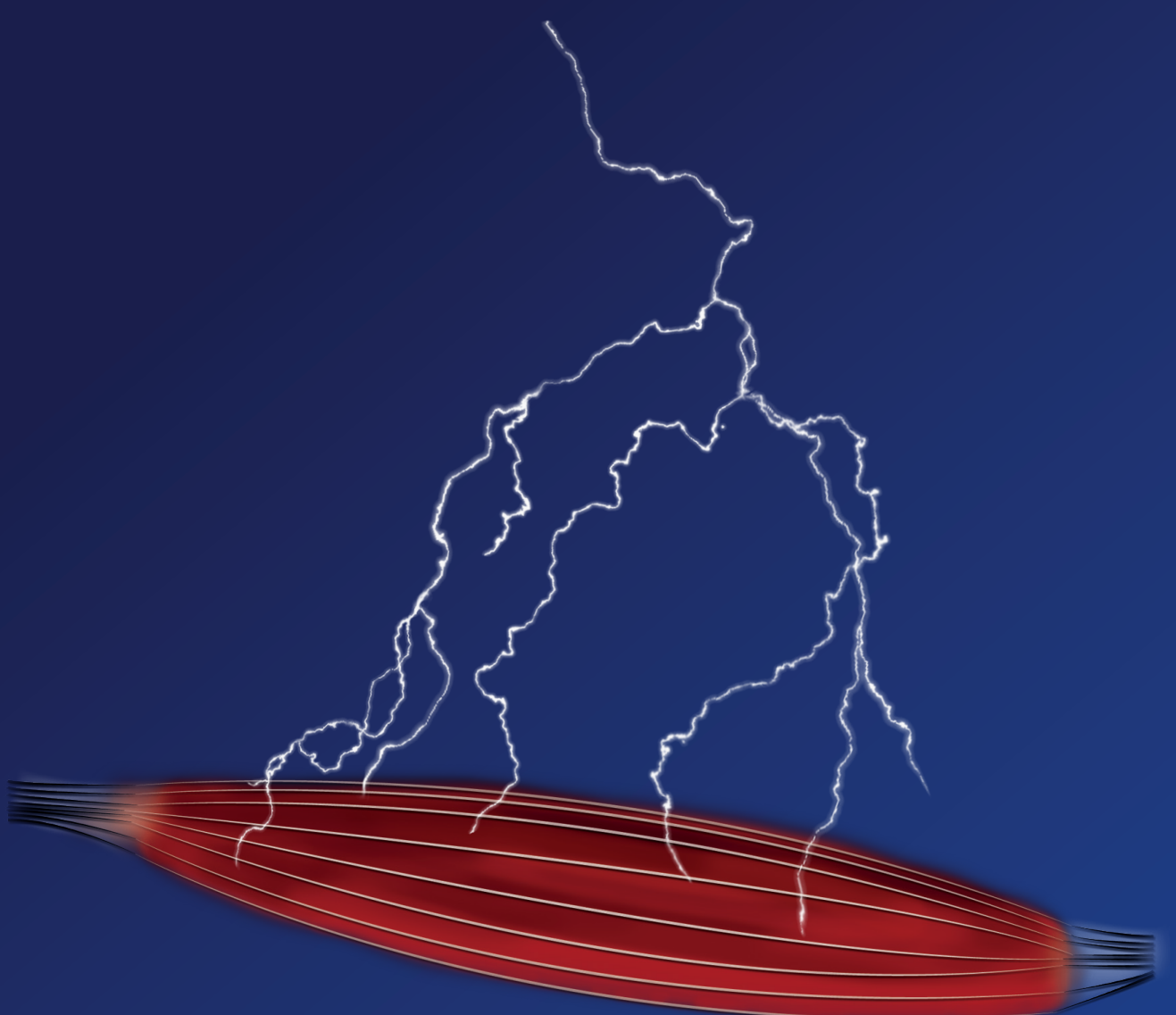

Maurice J.H. Sillen 

Neuromuscular electrical stimulation in dyspneic COPD patients:

a new training modality 
Copyright @ Maurice Sillen, Maastricht 2014

ISBN 9789461593177

Lay out: Tiny Wouters

Cover design: Kevin Derks

Production: Datawyse | Universitaire Pers Maastricht

The studies presented in this thesis were performed at $\mathrm{CIRO}^{+}$, center of expertise for chronic organ failure, Horn, the Netherlands.

This thesis was financially supported by $\mathrm{CIRO}^{+}$, center of expertise for chronic organ failure, the Netherlands; the Lung Foundation, Amersfoort, the Netherlands, Grant 3.4.09.024 and the Weijerhorst Foundation, Maastricht, the Netherlands.

Printing and distribution of this thesis was financially supported by $\mathrm{CIRO}^{+}$, center of expertise for chronic organ failure, Horn, the Netherlands; DJO Global Inc, Herentals, Belgium; GlaxoSmithKline, Zeist, the Netherlands, the Lung Foundation, Amersfoort, the Netherlands and Stichting Astma Bestrijding, Amsterdam, the Netherlands. 


\title{
Neuromuscular electrical stimulation in dyspneic COPD patients: a new training modality
}

\author{
PROEFSCHRIFT \\ ter verkrijging van de graad van doctor \\ aan de Universiteit Maastricht, \\ op gezag van de Rector Magnificus, Prof. dr. L.L.G. Soete, \\ volgens het besluit van het College van Decanen, \\ in het openbaar te verdedigen op \\ donderdag 10 april 2014, om 14:00 uur \\ door \\ Maurice J.H. Sillen
}

Geboren op 29 november 1967 te Swalmen

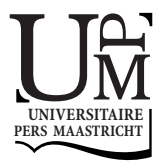




\section{Promotor}

Prof. dr. E.F.M. Wouters

\section{Copromotores}

Dr. M.A. Spruit (CIRO ${ }^{+}$, Horn)

Dr. F.M.E. Franssen (CIRO ${ }^{+}$, Horn)

\section{Beoordelingscommissie}

Prof. dr. H.P. Brunner-La Rocca (voorzitter)

Prof. dr. R. Gosselink (Katholieke Universiteit Leuven, België)

Prof. dr. M.K.C. Hesselink

Prof. dr. R.J.E.M. Smeets

Prof. dr. M. Vanderthommen (Université de Liège, België) 


\section{Table of contents}

$\begin{array}{lll}\text { Chapter } 1 \quad \text { General introduction } & 7\end{array}$

Chapter 2 Effects of neuromuscular electrical stimulation of muscles of 21 ambulation in patients with CHF or COPD: a systematic review of the English-language literature

Chapter 3 Metabolic and structural changes in lower-limb skeletal muscle following neuromuscular electrical stimulation:

a systematic review

Chapter 4 The metabolic response during resistance training and 79 neuromuscular electrical stimulation (NMES) in patients with COPD, a pilot study

Chapter 5 Oxygen uptake, ventilation, and symptoms during low-frequency versus high-frequency NMES in COPD, a pilot study

Chapter 6 Heterogeneity in clinical characteristics and co-morbidities in 101 dyspneic individuals with COPD GOLD D: Findings of the DICES trial

Chapter 7 Efficacy of lower-limb muscle training modalities in dyspneic individuals with chronic obstructive pulmonary disease:

results from the DICES trial

Chapter $8 \quad$ Metabolic load during strength training or NMES in individuals 149 with COPD: results from the DICES trial

Chapter 9 General discussion

Summary

Samenvatting

Dankwoord

Curriculum vitae

List of publications 



\section{CHAPTER 1}

General introduction 
Chapter 1 


\section{CHRONIC OBSTRUCTIVE PULMONARY DISEASE (COPD)}

COPD is defined as a preventable and treatable disease, characterized by persistent airflow limitation that is usually progressive and associated with an enhanced chronic inflammatory response in the airways and the lung to noxious particles or gases (1). The most important factor for the development and progression of COPD is cigarette smoking (2). The chronic airflow limitation characteristic of COPD is caused by a mixture of small airways disease (obstructive bronchiolitis) and parenchymal destruction (emphysema) (1). Clinically, COPD is characterized by chronic symptoms of progressive dyspnea, cough and sputum production. In symptomatic subjects, the diagnosis of COPD is confirmed by the presence of post-bronchodilator forced expiratory volume in the first second $\left(\mathrm{FEV}_{1}\right) /$ forced vital capacity $\left.(\mathrm{FVC})<0.70\right)(1)$. Traditionally, the Global Initiative for Chronic Obstructive Lung Disease (GOLD) graded severity of COPD according to the degree of airflow limitation into mild ( $\mathrm{FEV}_{1} \geq 80 \%$ predicted), moderate $\left(50 \% \leq \mathrm{FEV}_{1}<80 \%\right.$ predicted), severe $\left(30 \% \leq \mathrm{FEV}_{1}<50 \%\right.$ predicted) and very severe (FEV $1<30 \%$ predicted) (1). However, in the last decennium it has become clear that the degree of airflow limitation is a poor predictor of morbidity and mortality in individuals with COPD. Exacerbations and comorbidities contribute to the overall severity in individual patients (1). Therefore, the assessment of COPD was recently updated (1). In the updated GOLD strategy document, this assessment of disease severity is based on the combination of spirometric classification, symptoms and frequency of exacerbations. The predictive and prognostic properties of this new classification remain to be established (1).

COPD has been a major public health problem with sharply risen costs in the last twenty years (3). The current prevalence of moderate to very severe COPD is worldwide about $10 \%$ in people aged 40 years and older (4) and varies from country to country (5). Data of a large cohort study in the Netherlands show that the risk to be diagnosed with COPD in the coming 40 years is $12.7 \%$ for a 40 -year-old male and $8.3 \%$ for a 40 -year-old female (6). In 2002 COPD was reported as the fifth leading cause of death worldwide and it is estimated to become the fourth in 2030 (7). Similarly, from its ranking as the $12^{\text {th }}$-leading cause of disability-adjusted life years in 1990 , it is projected to become the fifth by 2020 (8).

In the last decades, it became clear that COPD is more than a respiratory disease characterized by the presence of chronic airflow limitation. Extra-pulmonary features, including skeletal muscle dysfunction and weight loss, and co-occurring diseases, such as cardiovascular disease, psychological conditions and metabolic abnormalities are common in these patients. Importantly, it has been shown that these systemic features of COPD contribute to morbidity and mortality. 


\section{SKELETAL MUSCLE DYSFUNCTION AND PHYSICAL INACTIVITY IN COPD}

Peripheral muscle strength $(9,10)$, endurance $(11,12)$ and fatigability $(13)$ are reduced in patients with COPD compared with healthy matched controls. Skeletal muscle weakness is present in one-third of patients with COPD (14), while even higher prevalences were reported in patients with very severe airflow limitation and patients with severe dyspnea. Skeletal muscle dysfunction is directly related to exercise intolerance (15), increased health care utilization (16) and mortality in patients with COPD (17).

A decreased peripheral muscle strength in COPD can largely be explained by a reduction in quadriceps muscle mass and cross-sectional area (CSA) (18-20). The loss in muscle mass has predominantly been observed in type Ila/IIx and type IIx fibers and associated with a disturbed metabolic capacity (21). A decreased muscle endurance is related to the loss of fatigue resistant type I fibers $(22,23)$. Although there are several factors potentially contributing to alterations in the skeletal muscle mass and function in COPD, including chronic or intermittent hypoxemia, hypercapnia, medications (e.g. corticosteroids), semi-starvation, hormonal imbalance, systemic or local inflammation, oxidative stress and genetic susceptibility, physical inactivity is perhaps the most obvious factor (24). Patients with COPD often adopt a sedentary lifestyle due to dyspnea and this can lead to deconditioning and muscle dysfunction, resulting in aggravated exercise-induced dyspnea (25). Anaerobic muscle metabolism results in early lactate release and increased carbon dioxide production, which stimulates ventilation and worsens breathlessness $(25,26)$. During domestic activities of daily life, COPD patients with high dyspnea grades used a significantly higher proportion of their peak aerobic capacity and ventilation accompanied with increased dyspnea scores (27). Physical inactivity is in patients with COPD significantly correlated with a decreased quadriceps muscle strength (28) and a decreased muscle endurance (11). Furthermore, physical activity is protective against hospital admissions (29). In a German cohort of 170 patients, it was demonstrated that objectively measured physical activity is the best predictor of all-cause mortality in patients with COPD (30). Given the impact of physical inactivity on symptoms, skeletal muscle dysfunction and exercise performance, increasing daily physical activity levels has become an important goal in the pharmacological and non-pharmacological management of patients with COPD. Pulmonary rehabilitation is considered as the most important non-pharmacological treatment strategy for disabled patients (31). 


\section{PULMONARY REHABILITATION}

Pulmonary rehabilitation is a comprehensive intervention based on a thorough patient assessment followed by patient-tailored therapies which include, but are not limited to, exercise training, education and behavior change, designed to improve the physical and psychological condition of people with chronic respiratory disease and to promote the long-term adherence of health-enhancing behaviors (31). Pulmonary rehabilitation programs involve initial and ongoing assessments (including disease severity, complexity and comorbidities), exercise training, education, nutritional intervention and psychosocial support and should be individualized to the unique needs of the patient (31,32).

Exercise-based rehabilitation programs are able to improve lower-limb muscle strength and endurance, functional exercise performance, physical activity levels and health status in patients with COPD (33-35). Exercise training has been shown to be the cornerstone of pulmonary rehabilitation programs (36) and typically consist of wholebody endurance or interval training (37). The preferred exercise prescription for endurance exercise training to maximize physiologic benefits (i.e., exercise tolerance, muscle function and bioenergetics) is an intensity of $>60 \%$ of the peak work load with a frequency of 3-5 times per week $(31,38)$. Interval training is a modification of endurance training where high-intensity exercise is interspersed regularly with periods of lower intensity exercise (31). Both training modalities resulted in significant improvements in exercise capacity and health status in patients with COPD without significant differences between both modalities (37). These improvements were accompanied with improvements in quadriceps fatigability (39) and significantly improved cross-sectional areas of type I and type lla fibers in the quadriceps muscle (40) after both types of training. Unfortunately, patients are not always able to perform high intensity endurance exercises due to exercise-induced dyspnea and fatigue (41). A possible explanation is that aerobic exercise training causes a relatively high claim on the peak aerobic capacity of patients with COPD (42). Therefore, other interventions should be considered for an active training strategy of severely dyspneic patients in a pulmonary rehabilitation program. Muscle strength training and transcutaneous neuromuscular electrical stimulation (NMES) may be suitable rehabilitative options. Ventilatory responses have been shown to be acceptable low during muscle strength training compared to aerobic exercise training in patients with COPD (42) and during NMES in healthy subjects (43). Moreover, COPD patients with increased dyspnea scores are less likely to complete a pulmonary rehabilitation program (44). Therefore, for dyspneic patients with COPD with muscle weakness, muscle strength training or NMES may be effective alternative rehabilitative modalities (45). 


\section{MUSCLE STRENGTH TRAINING}

Muscle strength training is exercise that causes muscles to work or hold against an applied force or weight (46). General principles of progression in muscle strength training are progressive overload, specificity and variation (47). Important program parameters are intensity, volume, exercise selection and order, rest periods between sets and frequency (47). Force generation is positively related to muscle fiber CSA (48) and further dependent on motor unit activation (49). Motor units are recruited according to the size principle, which is the recruitment threshold that typically involves the activation of the slower (lower force-producing) motor units before the faster (higher force-producing) units (49). The magnitude of strength enhancement is largely dependent on enhanced neural function (e.g., greater recruitment, rate of discharge) (50), increased muscle CSA (51-53), changes in muscle architecture (54) and possible adaptations to increased metabolites (55).

Muscle strength training has been shown to result in significant improvements in lowerlimb muscle function, exercise capacity and health status in individuals with (severely) disabled COPD (56). Progressive muscle strength training can also improve performance of tasks such as stair climbing and rising from sitting in patients with COPD (56). Training frequencies of 2 to 3 days per week, with 1 to 3 sets of 8-10 repetitions using loads progressing from $50-85 \%$ of the current 1 repetition maximum have been demonstrated efficacy, revealing substantial improvements in muscle strength and size with no untoward effects in patients with mild to moderate COPD (57-59). Compared to endurance training, muscle strength training led to similar improvements in functional exercise capacity $(60,61)$ and to similar $(60)$ or larger $(62,63)$ improvements in skeletal muscle strength in patients with COPD. Moreover, progressive muscle strength training protocols appeared feasible and safe for people with COPD when performed under supervision in an outpatient clinic, with few reported adverse events (56).

\section{NEUROMUSCULAR ELECTRICAL STIMULATION (NMES)}

NMES involves the application of a series of intermittent stimuli (stimulation-rest cycles) with the aid of percutaneous electrodes over superficial skeletal muscles, with the main objective to trigger visible muscle contractions due to the activation of the intramuscular nerve branches (64-66). For a number of years NMES has been used by physical therapists in research and clinical settings as a rehabilitation/training method to limit the atrophy that occurs with immobilization $(65,66)$. Mainly, the quadriceps femoris muscle has been stimulated with improvements in strength in normal and impaired muscles compared with doing no exercise in healthy subjects $(67,68)$ and sportsmen (69). NMES has also been proven as a successful training strategy for the preservation or recovery of muscle mass and function during prolonged periods of 
disuse or immobilization $(70,71)$ and for the improvement of muscle function in different patient populations, such as orthopedic (72) or neurological patients (73).

An interesting aspect of NMES is the activation order of motor units, which is quite different from the physiological recruitment pattern according to the size principle (49). Contradictory findings on motor unit recruitment order have been found (65). Some studies suggest preferential or selective activation of fast motor units with NMES $(74,75)$, whereas others suggest that motor unit recruitment during NMES reflects a non-selective, spatially fixed, and temporally synchronous pattern rather than in a reversal of the physiological voluntary recruitment order (76). These diverse results could have been related to differences in protocols and stimulated muscles (66). Another difference with voluntary contractions is that NMES activates the muscle to a greater extent under identical technical conditions (77). At identical levels of workload ( $10 \%$ of the quadriceps maximum isometric voluntary torque), the muscle reaches higher values in blood flow and oxygen consumption during NMES compared with voluntary contractions (77). NMES training sessions generally last 10-30 minutes during a 4- to 5-week period that involves 20-25 sessions to increase peripheral muscle function (66). Two types of NMES frequencies can be distinguished: high-frequency NMES (HF-NMES, $\geq 50$ Hertz); and low-frequency NMES (LF-NMES, <50 Hertz) $(65,66$, $78,79)$. Frequencies of 50 Hertz and above induce a fused tetanus $(80,81)$ and generate higher torques than low frequencies (82).

Although several trials had studied the functional and structural effects of NMES in COPD, the literature had not been systematically reviewed. To date, a number of studies have been published about the effects of NMES on gains in muscle performance, activation of motor units and/or muscle energetics (65). However, effects of NMES on intramuscular changes have not been systematically reviewed yet. NMES is used in research and clinical settings as an intervention for the preservation or recovery of muscle mass and improvement of muscle function. Nonetheless, a head-to-head comparison between strength training and HF-NMES has not been done in individuals with severe COPD. Also, effects of LF-NMES at 15 Hertz, which increased exercise capacity in individuals with chronic heart failure (83), have not been studied in individuals with COPD. Even though most pulmonary rehabilitation studies describe the aimed exercise training load, the actual training load course has been described scarcely in individuals with COPD. Furthermore, the metabolic load, expressed as oxygen consumption and ventilation, has never been assessed during NMES in patients with COPD. 


\section{AIMS OF THE THESIS}

The general aim of this thesis was to study the effects of NMES in severely dyspneic patients with COPD, compared with muscle strength training, on functional outcome measures and ventilatory responses.

Chapter 2 aimed to present a systematic review on the effects of NMES on the muscles of ambulation in patients with congestive heart failure or COPD, paying particular attention to the effects on peripheral muscle strength, exercise capacity and diseasespecific health status.

In order to increase understanding of the mechanisms of functional improvements following NMES, the aim of chapter $\mathbf{3}$ was to systematically review changes in enzyme activity, muscle fiber type composition and muscle fiber size in human lower-limb skeletal muscles following only NMES.

Because there is substantial interest in training modalities that do not evoke dyspnea in severely dyspneic patients with COPD, the aim of chapter $\mathbf{4}$ and $\mathbf{5}$ was to assess the metabolic load during one session of muscle strength training and one session of NMES. The metabolic load, expressed as oxygen consumption and ventilation, and symptom scores for dyspnea and fatigue were measured during these training modalities.

The aim of chapter 6 was to assess the clinical heterogeneity of a well-defined subgroup of very severe COPD patients with high symptoms (GOLD D) and a clinical indication for NMES, including possible gender differences.

Chapter 7 aimed to study the effects of HF-NMES, LF-NMES and muscle strength training as part of a comprehensive interdisciplinary pulmonary rehabilitation program in patients with COPD cardinal individual disease characteristics such as debilitating dyspnea and quadriceps weakness.

The aim of chapter 8 was to study the metabolic load over time during HF-NMES, LFNMES and strength training in individuals with COPD.

In chapter 9 the aim was to put the changes in skeletal muscle strength and exercise capacity following HF-NMES, LF-NMES and strength training into perspective, including the metabolic load during NMES and strength training, and to discuss future directions. 


\section{OUTLINE OF THE THESIS}

In chapter 2 effects of NMES of the lower-limb muscles on strength, exercise capacity and disease-specific health status in patients with CHF or COPD were systematically reviewed.

Chapter 3 presents a systematic review on the changes in enzyme activity, muscle fiber type composition and muscle fiber size in human lower-limb skeletal muscles following NMES.

Chapter 4 is a pilot study on the metabolic response (expressed as oxygen uptake and ventilation) during one session of muscle strength training and one session of HFNMES.

In chapter 5 oxygen uptake, ventilation and symptoms of dyspnea and fatigue during one session of HF-NMES and one session of LF-NMES were studied.

Chapter 6 focused on the clinical heterogeneity in a well-defined subgroup of individuals with COPD GOLD D, including gender differences.

Chapter 7 presents a prospective randomized controlled trial, which was designed to compare effects of HF-NMES, LF-NMES and high-intensity muscle strength training in dyspneic COPD patients with lower limb muscle dysfunction entering a comprehensive interdisciplinary pulmonary rehabilitation program.

In chapter 8 the metabolic load, expressed as oxygen uptake and ventilation, during HF-NMES, LF-NMES and high-intensity muscle strength training was measured.

In chapter 9 changes in skeletal muscle strength and exercise capacity following HFNMES, LF-NMES and strength training and the metabolic load during these interventions are discussed. 


\section{REFERENCES}

1. Vestbo J, Hurd SS, Agusti AG, Jones PW, Vogelmeier C, Anzueto A, et al. Global strategy for the diagnosis, management, and prevention of chronic obstructive pulmonary disease: GOLD executive summary. Am J Respir Crit Care Med. 2013;187(4):347-65.

2. Forey BA, Thornton AJ, Lee PN. Systematic review with meta-analysis of the epidemiological evidence relating smoking to COPD, chronic bronchitis and emphysema. BMC Pulm Med. 2011;11:36.

3. Blanchette CM, Dalal AA, Mapel D. Changes in COPD demographics and costs over 20 years. Journal of medical economics. 2012;15(6):1176-82.

4. Buist AS, McBurnie MA, Vollmer WM, Gillespie S, Burney P, Mannino DM, et al. International variation in the prevalence of COPD (the BOLD Study): a population-based prevalence study. Lancet. 2007;370(9589):741-50.

5. Atsou K, Chouaid C, Hejblum G. Variability of the chronic obstructive pulmonary disease key epidemiological data in Europe: systematic review. BMC medicine. 2011;9:7.

6. Afonso AS, Verhamme KM, Sturkenboom MC, Brusselle GG. COPD in the general population: prevalence, incidence and survival. Respir Med. 2011;105(12):1872-84.

7. Mathers CD, Loncar D. Projections of global mortality and burden of disease from 2002 to 2030. PLoS Med. 2006;3(11):e442.

8. Lopez AD, Shibuya K, Rao C, Mathers CD, Hansell AL, Held LS, et al. Chronic obstructive pulmonary disease: current burden and future projections. Eur Respir J. 2006;27(2):397-412.

9. Bernard S, LeBlanc P, Whittom F, Carrier G, Jobin J, Belleau R, et al. Peripheral muscle weakness in patients with chronic obstructive pulmonary disease. Am J Respir Crit Care Med. 1998;158(2):629-34.

10. Man WD, Soliman MG, Nikoletou D, Harris ML, Rafferty GF, Mustfa N, et al. Non-volitional assessment of skeletal muscle strength in patients with chronic obstructive pulmonary disease. Thorax. 2003;58(8):665-9.

11. Serres I, Gautier V, Varray A, Prefaut C. Impaired skeletal muscle endurance related to physical inactivity and altered lung function in COPD patients. Chest. 1998;113(4):900-5.

12. Allaire J, Maltais F, Doyon JF, Noel M, LeBlanc P, Carrier G, et al. Peripheral muscle endurance and the oxidative profile of the quadriceps in patients with COPD. Thorax. 2004;59(8):673-8.

13. Mador MJ, Bozkanat E, Kufel TJ. Quadriceps fatigue after cycle exercise in patients with COPD compared with healthy control subjects. Chest. 2003;123(4):1104-11.

14. Seymour JM, Spruit MA, Hopkinson NS, Natanek SA, Man WD, Jackson A, et al. The prevalence of quadriceps weakness in COPD and the relationship with disease severity. Eur Respir J. 2010;36(1):81-8.

15. Gosselink R, Troosters T, Decramer M. Peripheral muscle weakness contributes to exercise limitation in COPD. Am J Respir Crit Care Med. 1996;153(3):976-80.

16. Decramer M, Gosselink R, Troosters T, Verschueren M, Evers G. Muscle weakness is related to utilization of health care resources in COPD patients. Eur Respir J. 1997;10(2):417-23.

17. Swallow EB, Reyes D, Hopkinson NS, Man WD, Porcher R, Cetti EJ, et al. Quadriceps strength predicts mortality in patients with moderate to severe chronic obstructive pulmonary disease. Thorax. 2007;62(2):115-20.

18. Mathur S, Takai KP, Macintyre DL, Reid D. Estimation of thigh muscle mass with magnetic resonance imaging in older adults and people with chronic obstructive pulmonary disease. Phys Ther. 2008;88(2):219-30.

19. Seymour JM, Ward K, Sidhu PS, Puthucheary Z, Steier J, Jolley CJ, et al. Ultrasound measurement of rectus femoris cross-sectional area and the relationship with quadriceps strength in COPD. Thorax. 2009;64(5):418-23.

20. Marquis K, Debigare R, Lacasse $Y$, LeBlanc P, Jobin J, Carrier G, et al. Midthigh muscle cross-sectional area is a better predictor of mortality than body mass index in patients with chronic obstructive pulmonary disease. Am J Respir Crit Care Med. 2002;166(6):809-13.

21. Gosker HR, Engelen MP, van Mameren H, van Dijk PJ, van der Vusse GJ, Wouters EF, et al. Muscle fiber type IIX atrophy is involved in the loss of fat-free mass in chronic obstructive pulmonary disease. Am J Clin Nutr. 2002;76(1):113-9. 
22. Gosker HR, Zeegers MP, Wouters EF, Schols AM. Muscle fibre type shifting in the vastus lateralis of patients with COPD is associated with disease severity: a systematic review and meta-analysis. Thorax. 2007;62(11):944-9.

23. Whittom F, Jobin J, Simard PM, Leblanc P, Simard C, Bernard S, et al. Histochemical and morphological characteristics of the vastus lateralis muscle in patients with chronic obstructive pulmonary disease. Med Sci Sports Exerc. 1998;30(10):1467-74.

24. Man WD, Kemp P, Moxham J, Polkey MI. Skeletal muscle dysfunction in COPD: clinical and laboratory observations. Clin Sci (Lond). 2009;117(7):251-64.

25. Polkey MI, Moxham J. Attacking the disease spiral in chronic obstructive pulmonary disease: an update. Clin Med. 2011;11(5):461-4.

26. Donaldson AV, Maddocks M, Martolini D, Polkey MI, Man WD. Muscle function in COPD: a complex interplay. Int J Chron Obstruct Pulmon Dis. 2012;7:523-35.

27. Vaes AW, Wouters EF, Franssen FM, Uszko-Lencer NH, Stakenborg KH, Westra M, et al. Task-related oxygen uptake during domestic activities of daily life in patients with COPD and healthy elderly subjects. Chest. 2011;140(4):970-9.

28. Pitta F, Troosters T, Spruit MA, Probst VS, Decramer M, Gosselink R. Characteristics of physical activities in daily life in chronic obstructive pulmonary disease. Am J Respir Crit Care Med. 2005;171(9):972-7.

29. Garcia-Aymerich J, Farrero E, Felez MA, Izquierdo J, Marrades RM, Anto JM. Risk factors of readmission to hospital for a COPD exacerbation: a prospective study. Thorax. 2003;58(2):100-5.

30. Waschki B, Kirsten A, Holz O, Muller KC, Meyer T, Watz H, et al. Physical activity is the strongest predictor of all-cause mortality in patients with COPD: a prospective cohort study. Chest. 2011;140(2):331-42.

31. Spruit MA, Singh SJ, Garvey C, ZuWallack R, Nici L, Rochester C, et al. An Official American Thoracic Society / European Respiratory Society Statement: Key concepts and advances in pulmonary rehabilitation. Am J Respir Crit Care Med. 2013;in press.

32. Nici L, Donner C, Wouters E, Zuwallack R, Ambrosino N, Bourbeau J, et al. American Thoracic Society/European Respiratory Society statement on pulmonary rehabilitation. Am J Respir Crit Care Med. 2006;173(12):1390-413.

33. Lacasse Y, Goldstein R, Lasserson TJ, Martin S. Pulmonary rehabilitation for chronic obstructive pulmonary disease. Cochrane Database Syst Rev. 2006(4):CD003793.

34. Pitta F, Troosters T, Probst VS, Langer D, Decramer M, Gosselink R. Are patients with COPD more active after pulmonary rehabilitation? Chest. 2008;134(2):273-80.

35. Walker PP, Burnett A, Flavahan PW, Calverley PM. Lower limb activity and its determinants in COPD. Thorax. 2008;63(8):683-9.

36. Spruit MA, Troosters T, Trappenburg JC, Decramer M, Gosselink R. Exercise training during rehabilitation of patients with COPD: a current perspective. Patient Educ Couns. 2004;52(3):243-8.

37. Beauchamp MK, Nonoyama M, Goldstein RS, Hill K, Dolmage TE, Mathur S, et al. Interval versus continuous training in individuals with chronic obstructive pulmonary disease--a systematic review. Thorax. 2010;65(2):157-64.

38. Garber CE, Blissmer B, Deschenes MR, Franklin BA, Lamonte MJ, Lee IM, et al. American College of Sports Medicine position stand. Quantity and quality of exercise for developing and maintaining cardiorespiratory, musculoskeletal, and neuromotor fitness in apparently healthy adults: guidance for prescribing exercise. Med Sci Sports Exerc. 2011;43(7):1334-59.

39. Mador MJ, Krawza M, Alhajhusian A, Khan Al, Shaffer M, Kufel TJ. Interval training versus continuous training in patients with chronic obstructive pulmonary disease. J Cardiopulm Rehabil Prev. 2009;29(2):126-32.

40. Vogiatzis I, Terzis G, Nanas S, Stratakos G, Simoes DC, Georgiadou O, et al. Skeletal muscle adaptations to interval training in patients with advanced COPD. Chest. 2005;128(6):3838-45.

41. Maltais F, LeBlanc P, Jobin J, Berube C, Bruneau J, Carrier L, et al. Intensity of training and physiologic adaptation in patients with chronic obstructive pulmonary disease. Am J Respir Crit Care Med. 1997;155(2):555-61.

42. Probst VS, Troosters T, Pitta F, Decramer M, Gosselink R. Cardiopulmonary stress during exercise training in patients with COPD. Eur Respir J. 2006;27(6):1110-8. 
43. Theurel J, Lepers R, Pardon L, Maffiuletti NA. Differences in cardiorespiratory and neuromuscular responses between voluntary and stimulated contractions of the quadriceps femoris muscle. Respiratory physiology \& neurobiology. 2007;157(2-3):341-7.

44. Hogg L, Garrod R, Thornton H, McDonnell L, Bellas H, White P. Effectiveness, attendance, and completion of an integrated, system-wide pulmonary rehabilitation service for COPD: prospective observational study. COPD. 2012;9(5):546-54.

45. Spruit MA, Wouters EF. New modalities of pulmonary rehabilitation in patients with chronic obstructive pulmonary disease. Sports Med. 2007;37(6):501-18.

46. American College of Sports Medicine Position Stand. Exercise and physical activity for older adults. Med Sci Sports Exerc. 1998;30(6):992-1008.

47. Kraemer WJ, Ratamess NA. Fundamentals of resistance training: progression and exercise prescription. Med Sci Sports Exerc. 2004;36(4):674-88.

48. Finer JT, Simmons RM, Spudich JA. Single myosin molecule mechanics: piconewton forces and nanometre steps. Nature. 1994;368(6467):113-9.

49. Henneman E, Somjen G, Carpenter DO. Functional Significance of Cell Size in Spinal Motoneurons. J Neurophysiol. 1965;28:560-80.

50. Sakamoto A, Sinclair PJ. Effect of movement velocity on the relationship between training load and the number of repetitions of bench press. J Strength Cond Res. 2006;20(3):523-7.

51. Alway SE, Grumbt WH, Gonyea WJ, Stray-Gundersen J. Contrasts in muscle and myofibers of elite male and female bodybuilders. J Appl Physiol. 1989;67(1):24-31.

52. McCall GE, Byrnes WC, Dickinson A, Pattany PM, Fleck SJ. Muscle fiber hypertrophy, hyperplasia, and capillary density in college men after resistance training. J Appl Physiol. 1996;81(5):2004-12.

53. Staron RS, Karapondo DL, Kraemer WJ, Fry AC, Gordon SE, Falkel JE, et al. Skeletal muscle adaptations during early phase of heavy-resistance training in men and women. J Appl Physiol. 1994;76(3):1247-55.

54. Kawakami Y, Abe T, Fukunaga T. Muscle-fiber pennation angles are greater in hypertrophied than in normal muscles. J Appl Physiol. 1993;74(6):2740-4.

55. Shinohara M, Kouzaki M, Yoshihisa T, Fukunaga T. Efficacy of tourniquet ischemia for strength training with low resistance. Eur J Appl Physiol Occup Physiol. 1998;77(1-2):189-91.

56. O'Shea SD, Taylor NF, Paratz JD. Progressive resistance exercise improves muscle strength and may improve elements of performance of daily activities for people with COPD: a systematic review. Chest. 2009;136(5):1269-83.

57. Bernard S, Whittom F, Leblanc P, Jobin J, Belleau R, Berube C, et al. Aerobic and strength training in patients with chronic obstructive pulmonary disease. Am J Respir Crit Care Med. 1999;159(3):896-901.

58. Clark CJ, Cochrane LM, Mackay E, Paton B. Skeletal muscle strength and endurance in patients with mild COPD and the effects of weight training. Eur Respir J. 2000;15(1):92-7.

59. Simpson K, Killian K, McCartney N, Stubbing DG, Jones NL. Randomised controlled trial of weightlifting exercise in patients with chronic airflow limitation. Thorax. 1992;47(2):70-5.

60. Spruit MA, Gosselink R, Troosters T, De Paepe K, Decramer M. Resistance versus endurance training in patients with COPD and peripheral muscle weakness. Eur Respir J. 2002;19(6):1072-8.

61. Wurtemberger G, Bastian K. [Functional effects of different training in patients with COPD]. Pneumologie. 2001;55(12):553-62. Funktionelle Effekte unterschiedlicher Trainingsformen bei Patienten mit COPD.

62. Normandin EA, McCusker C, Connors M, Vale F, Gerardi D, ZuWallack RL. An evaluation of two approaches to exercise conditioning in pulmonary rehabilitation. Chest. 2002;121(4):1085-91.

63. Ortega F, Toral J, Cejudo P, Villagomez R, Sanchez H, Castillo J, et al. Comparison of effects of strength and endurance training in patients with chronic obstructive pulmonary disease. Am J Respir Crit Care Med. 2002;166(5):669-74.

64. Hultman E, Sjoholm H, Jaderholm-Ek I, Krynicki J. Evaluation of methods for electrical stimulation of human skeletal muscle in situ. Pflugers Arch. 1983;398(2):139-41.

65. Maffiuletti NA. Physiological and methodological considerations for the use of neuromuscular electrical stimulation. Eur J Appl Physiol. 2010;110(2):223-34.

66. Vanderthommen $M$, Duchateau J. Electrical stimulation as a modality to improve performance of the neuromuscular system. Exerc Sport Sci Rev. 2007;35(4):180-5. 
67. Bax L, Staes F, Verhagen A. Does neuromuscular electrical stimulation strengthen the quadriceps femoris? A systematic review of randomised controlled trials. Sports Med. 2005;35(3):191-212.

68. Filipovic A, Kleinoder H, Dormann U, Mester J. Electromyostimulation--a systematic review of the influence of training regimens and stimulation parameters on effectiveness in electromyostimulation training of selected strength parameters. J Strength Cond Res. 2011;25(11):3218-38.

69. Maffiuletti NA. The use of electrostimulation exercise in competitive sport. International journal of sports physiology and performance. 2006;1(4):406-7.

70. Gibson JN, Smith K, Rennie MJ. Prevention of disuse muscle atrophy by means of electrical stimulation: maintenance of protein synthesis. Lancet. 1988;2(8614):767-70.

71. Snyder-Mackler L, Delitto A, Bailey SL, Stralka SW. Strength of the quadriceps femoris muscle and functional recovery after reconstruction of the anterior cruciate ligament. A prospective, randomized clinical trial of electrical stimulation. J Bone Joint Surg Am. 1995;77(8):1166-73.

72. Kim KM, Croy T, Hertel J, Saliba S. Effects of neuromuscular electrical stimulation after anterior cruciate ligament reconstruction on quadriceps strength, function, and patient-oriented outcomes: a systematic review. J Orthop Sports Phys Ther. 2010;40(7):383-91.

73. Chae J, Sheffler L, Knutson J. Neuromuscular electrical stimulation for motor restoration in hemiplegia. Topics in stroke rehabilitation. 2008;15(5):412-26.

74. Cabric M, Appell HJ, Resic A. Fine structural changes in electrostimulated human skeletal muscle. Evidence for predominant effects on fast muscle fibres. Eur J Appl Physiol Occup Physiol. 1988;57(1): 1-5.

75. Trimble $\mathrm{MH}$, Enoka RM. Mechanisms underlying the training effects associated with neuromuscular electrical stimulation. Phys Ther. 1991;71(4):273-80; discussion 80-2.

76. Bickel CS, Gregory CM, Dean JC. Motor unit recruitment during neuromuscular electrical stimulation: a critical appraisal. Eur J Appl Physiol. 2011;111(10):2399-407.

77. Vanderthommen M, Duteil S, Wary C, Raynaud JS, Leroy-Willig A, Crielaard JM, et al. A comparison of voluntary and electrically induced contractions by interleaved $1 \mathrm{H}$ - and 31P-NMRS in humans. J Appl Physiol. 2003;94(3):1012-24.

78. Vivodtzev I, Lacasse Y, Maltais F. Neuromuscular electrical stimulation of the lower limbs in patients with chronic obstructive pulmonary disease. J Cardiopulm Rehabil Prev. 2008;28(2):79-91.

79. Grimby L, Hannerz J. Firing rate and recruitment order of toe extensor motor units in different modes of voluntary conraction. J Physiol. 1977;264(3):865-79.

80. Taylor A, Stephens JA. Study of human motor unit contractions by controlled intramuscular microstimulation. Brain research. 1976;117(2):331-5.

81. Edwards RH, Young A, Hosking GP, Jones DA. Human skeletal muscle function: description of tests and normal values. Clinical science and molecular medicine. 1977;52(3):283-90.

82. Kramer JF. Effect of electrical stimulation current frequencies on isometric knee extension torque. Phys Ther. 1987;67(1):31-8.

83. Nuhr MJ, Pette D, Berger R, Quittan M, Crevenna R, Huelsman M, et al. Beneficial effects of chronic low-frequency stimulation of thigh muscles in patients with advanced chronic heart failure. Eur Heart J. 2004;25(2):136-43. 



\section{CHAPTER 2}

Effects of neuromuscular electrical stimulation of

muscles of ambulation in patients with CHF or COPD:

a systematic review of the English-language literature

Maurice J.H. Sillen, Caroline M. Speksnijder, Rose-Miek A. Eterman, Paul P. Janssen, Scott S. Wagers, Emiel F.M. Wouters, Nicole H.M.K. Uszko-Lencer, Martijn A. Spruit

Chest 2009;13:44-61 


\section{ABSTRACT}

Despite optimal drug treatment, many patients with congestive heart failure (CHF) or COPD still experience disabling dyspnea, fatigue, and exercise intolerance. They also exhibit significant changes in body composition. Attempts to rehabilitate these patients are often futile because conventional exercise training modalities are limited by the severity of exertional dyspnea. Therefore, there is substantial interest in new training modalities that do not evoke dyspnea, such as transcutaneous neuromuscular electrical stimulation (NMES).

In this article, we systematically review the literature that addresses the effects of NMES applied to the muscles of ambulation. We focused on the effects of NMES on strength, exercise capacity, and disease-specific health status in patients with CHF or COPD. We also address the methodological quality of the reported studies as well as the safety of NMES.

Manuscripts published prior to December 2007 were identified by searching the Medline / PubMed, Embase, Cochrane Controlled Trials Register, CINAHL, and The Physical Therapy Evidence Database (PEDro) databases.

Fourteen trials were identified (nine trials that examined NMES in CHF patients, and five in COPD patients). PEDro scores for methodological quality of the trials were generally moderate to good. Many of the studies reported significant improvements in muscle strength, exercise capacity, and/or health status.

Nonetheless, the limited number of studies, the disparity in patient populations, and the variability in NMES methodology prohibit the use of meta-analysis. Yet, from the viewpoint of a systematic review NMES looks promising as a means of rehabilitating patients with COPD and CHF. There is at least sufficient evidence to warrant more large prospective randomized controlled trials. 


\section{INTRODUCTION}

Congestive heart failure (CHF) and chronic obstructive pulmonary disease (COPD) are major causes of morbidity and mortality worldwide (1-3). CHF is a syndrome in which the patient are typically characterized by breathlessness or fatigue, either at rest or during exertion; ankle swelling; and objective evidence of cardiac dysfunction at rest (4). COPD is a disease state characterized by airflow limitation that is not fully reversible. The airflow limitation is usually both progressive and associated with an abnormal inflammatory response of the lungs to noxious particles or gases (5). The overall prevalence of $\mathrm{CHF}$ is estimated at 3 to 20 per 1,000 population, although this exceeds 100 per 1,000 in those persons $>65$ years of age (6). The prevalence of COPD is estimated at 66 to 69 per 1,000 population (7). Both patients with CHF and those with COPD experience varying degrees of dyspnea and fatigue on exertion, and at rest (8). They are both progressive conditions with intermittent acute exacerbations that often necessitate a change in drug regimen and in some cases hospitalization $(9,10)$. Consequently, the burden on society is substantial (1).

Despite optimal drug treatment, many patients with clinically stable CHF or COPD still experience disabling dyspnea, fatigue, and exercise intolerance $(11,12)$. They also exhibit significant changes in body composition $(11,12)$. To date, the systemic features of CHF and COPD may, at least in part, contribute to the disease severity in individual patients. Consequently, they have taken a prominent position in the clinical phenotyping of patients with CHF or COPD (13). One of the most prominent changes is skeletal muscle atrophy $(14,15)$. Irrespective of the degree of primary organ failure, skeletal muscle atrophy is associated with skeletal muscle weakness, exercise intolerance, decreases in disease-specific health status and a poorer prognosis (16-23). Physical inactivity has been shown to be an important underlying factor which may at least in part contribute to skeletal muscular impairment in both CHF and COPD (24-26). For these reasons there is a rationale to include exercise training in any program of integrated care for $\operatorname{COPD}$ and $\operatorname{CHF}(27,28)$. Indeed, the benefit of exercise training in these patients has been demonstrated for all severities of disease $(27,29)$.

Conventional exercise training modalities, like endurance training and interval training in severely disabled patients with CHF or COPD are limited by the severity of exertional dyspnea they evoke (30). Therefore, there is substantial interest in new training modalities that do not evoke dyspnea, such as transcutaneous neuromuscular electrical stimulation (NMES) (31). NMES involves the application of an electrical current through electrodes placed on the skin over the targeted muscles, thereby depolarizing motor neurons and, in turn, inducing skeletal muscle contractions $(32,33)$ (Figure 2.1).

Although there have been a number of studies (34-36) on the functional and structural effects of NMES as well as its metabolic response (37), a systematic review has not to date been published. It has even been shown that NMES can relieve the perception of dyspnea (38). A narrative review of the use of NMES in COPD patients has been 
published (33), but the methodological quality of the included trials was not assessed. Here, we present a systematic review of the effect of NMES on muscles of ambulation in patients with CHF or COPD, paying particular attention to the effects on strength, exercise capacity and disease-specific health status. Additionally, the methodological quality will be assessed and the safety of NMES will be described.

Figure 2.1 Transcutaneous neuromuscular electrical stimulation of the $\mathrm{mm}$. quadriceps femoris in a male patient with chronic obstructive pulmonary disease (GOLD stage IV).

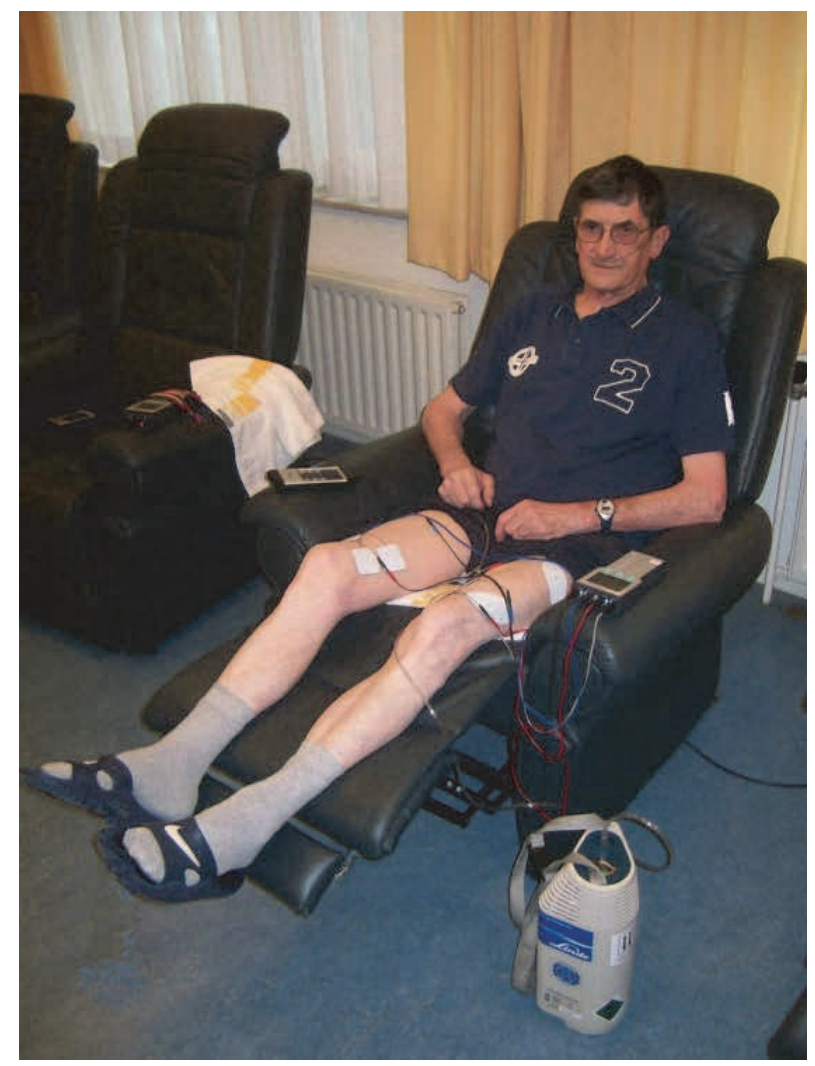

\section{MATERIALS AND METHODS}

\section{DATA SOURCES AND SEARCHES}

A computerized literature search was performed to identify relevant trials reported in the English language. The following several databases were used: Medline/PubMed 
(from 1966 to 2007); Embase (from 1974 to 2007); Cochrane Controlled Trials Register (from 1800 to 2007); CINAHL (from 1982 to 2007); and The Physical Therapy Evidence Database (PEDro) (from 1982 to 2007). Trials were collected up to December 2007. The complete search strategy can be found in Appendix 2.1. In addition, reference lists and citations of original articles were searched to identify additional articles that may contain information on the topic of interest.

\section{Article selection}

Only randomized controlled trials (RCTs) and controlled clinical trials (CCTS) were included that enrolled CHF (4) or COPD (39) patients and compared the effects of NMES on the muscles of ambulation alone or in combination with other exercise modalities. NMES is a method of augmenting muscle performance and can be used in the prevention and treatment of muscle atrophy (34-36). This electrical stimulation is composed of stimulation-rest cycles and is performed with the aid of percutaneous electrodes over skeletal muscles, situated in regard to muscle motor points (32). Trials had to compare NMES to placebo interventions (i.e. sham stimulation), to a usual rehabilitation program (e.g., ergometry cycling or treadmill walking), or to usual CHF/COPD care. A priori, editorials, reviews, case reports and congress abstracts were not included. Indeed, congress abstracts do not undergo a very stringent peer-review process. We reviewed the following outcome parameters: skeletal muscle function, exercise performance, and disease-specific health status.

\section{Data extraction}

A pre-designed data abstraction form was used to obtain data on trial design and relevant results. For each study Mr. Sillen noted characteristics of the patient group being studied, that is, the presence of CHF or COPD, age, body mass index (BMI), left ventricular ejection fraction (LVEF) or forced expiratory volume in the first second $\left(F_{1}\right.$ ), New York Heart Association (NYHA) classification, and aetiology of CHF (40), Global Initiative for Chronic Obstructive Lung Disease (GOLD) classification for COPD patients (39), experimental and control/placebo intervention(s) (i.e., type of intervention, pulse duration, pulse frequency, duty cycle and intensity of the used current, training intensity, session time, and duration in weeks), and the methods of outcome parameters and their results. Ms. Eterman and Dr. Spruit checked all data. Disagreements were resolved by consensus.

\section{Assessment OF METHOdological QUALITY}

The methodological quality of the identified trials was scored independently using the PEDro scale (Appendix 2.2). The PEDro scale is based on the Delphi list and "expert consensus" (41). The PEDro scale consists of 11 items/criteria (shown in Appendix 2.2) which can help the users of the scale to estimate the internal validity (criteria 2 to 9 ) and to identify whether the trial has sufficient statistical information or not to make the results interpretable (criteria 10 and 11). An additional criterion (criterion 1 ["Eligibility 
criteria"]) has been retained from the Delphi list and relates to the external validity. However, criterion 1 is not used in the calculation of the PEDro score. Each item receives either a "yes" or a "no" answer. All "yes" scores were summed, resulting in a maximum score of 10 points (41). Points were only awarded when a criterion was clearly satisfied. In cases where information was lacking or insufficient, the criterion was rated "no". A к coefficient was used to measure the level of interobserver agreement, using a method recommended for comparing level of agreement with categorical data along with their respective 95\% confidence intervals (42). Consensus was sought in case of disagreement. Previously, trials with a PEDro score of $\geq 6$ points were classified as "high-quality trials"; trials with a PEDro score $\leq 5$ points were classified as "low-quality trials" (43).

\section{Statistical analyses}

The use of meta-analytic techniques for data-analysis was not possible, because of the heterogeneity in NMES features (e.g., frequency, pulse duration, session time, total number of sessions), control group intervention (e.g., usual care, sham-stimulation, bicycle exercise training) and outcome parameters (e.g., peak workload, exercise duration, muscle strength, peak oxygen uptake). Therefore, the present authors were only able to systematically review the available peer-reviewed literature and to critically appraise the methodological quality and the overall findings. This was done in two separate meetings with Mr. Sillen, Ms. Eterman, Mr. Janssen, Dr. Wouters, Ms. Uszko-Lencer, and Dr. Spruit.

\section{RESULTS}

\section{SeARCH AND SELECTION}

Initially, 208 potentially relevant studies were identified by screening electronic databases. Of these trials, 195 were excluded due to the study design (no RCT or CCT), the studied population (no CHF or COPD as primary diagnosis) and/or the intervention (no NMES of muscles of ambulation). One trial was included by screening the reference lists. Finally, 14 trials were reviewed in detail (44-57). Nine RCTs studied the effects of NMES (alone or in combination with other exercise modalities) in CHF and five RCTs studied the effects of NMES (alone or in combination with other exercise modalities) in COPD (Figure 2.2). No CCTs were identified during the screening and selection process.

\section{Methodological quality of the trials}

The level of the interobserver agreement between the reviewers (Mr. Sillen and Ms. Eterman) was 0.89 (95\% Cl: 0.82-0.97; $\mathrm{p}<0.0001)$. PEDro scores ranged from 4 to 8 points in the CHF trials (median, 6 points). Three trials (33.3\%) (47-49) scored $<6$ points 
on the PEDro scale. In the COPD trials PEDro scores ranged from 6 to 8 points (median, 6 points). Failure to conceal allocation, and blinding of the patients, therapists and outcome assessors were the most prevalent methodological shortcomings (Table 2.1). There is substantial heterogeneity in the studied patient populations, NMES features, outcome parameters and the nature of the control groups (Tables 2.2, 2.3). For example, Vivodtzev and colleagues studied the effects of NMES and usual rehabilitation in COPD patients with an abnormal low BMI (56), whereas the mean BMI, if reported, was $>21 \mathrm{~kg} / \mathrm{m}^{2}$ in the other trials (Tables 2.2, 2.3). Additionally, patients were not always in a clinically stable condition during (52) and even at inclusion of the trial (57).

\section{Figure 2.2 Screening and selection process of trials.}

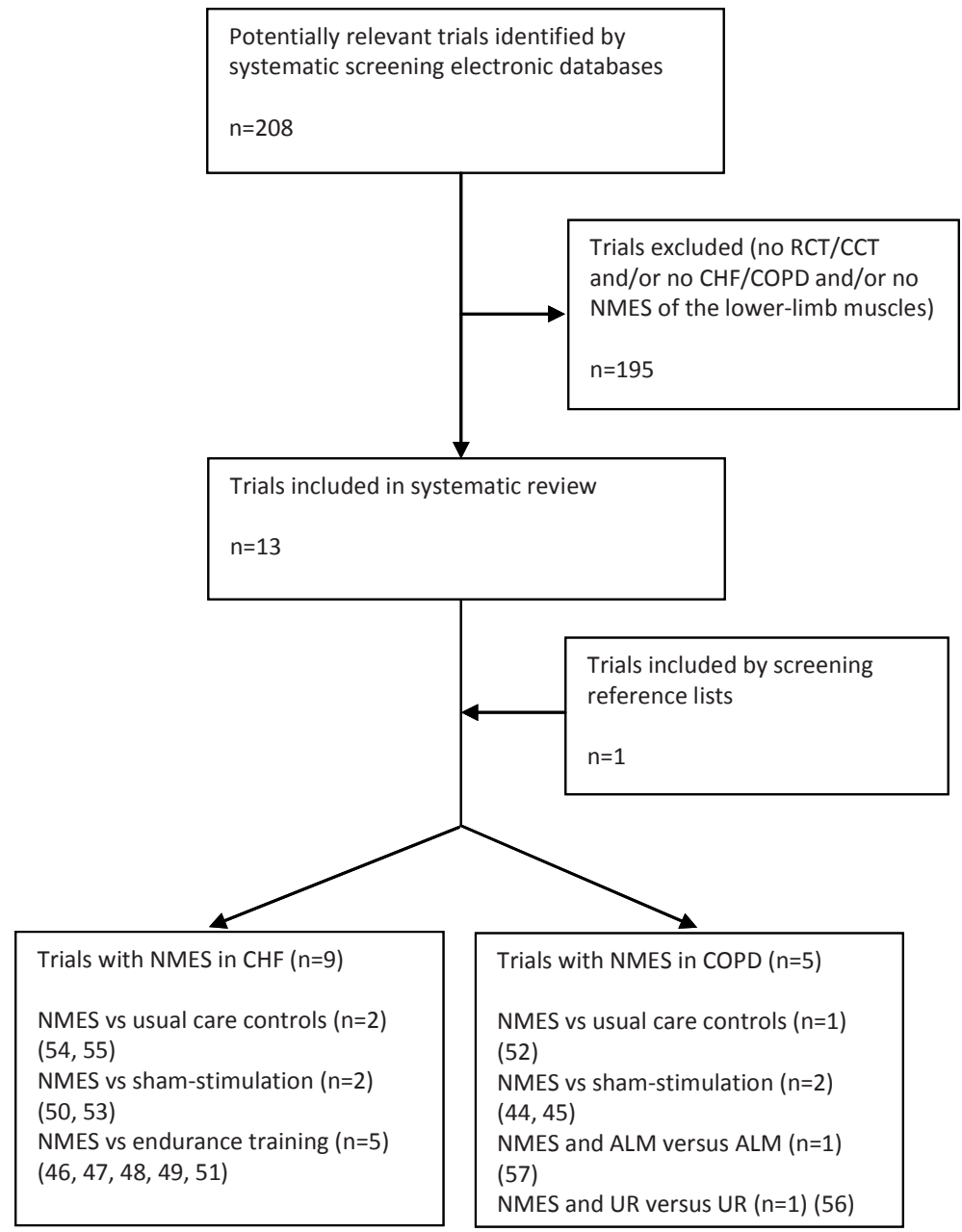

Abbreviations: (ALM=active limb mobilization; $C H F=$ chronic heart failure; $C O P D=c h r o n i c$ obstructive pulmonary disease; UR='usual' rehabilitation). 


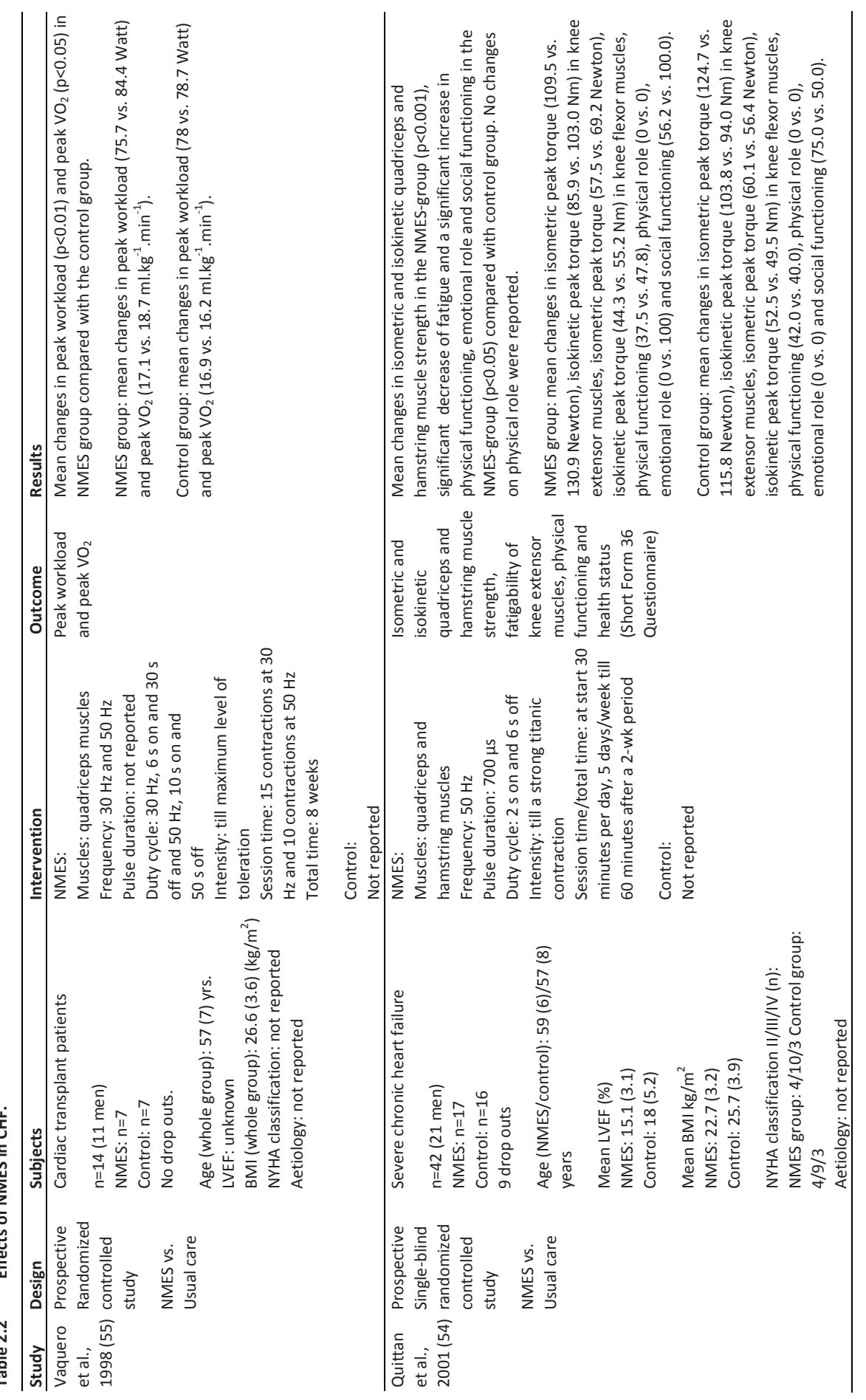




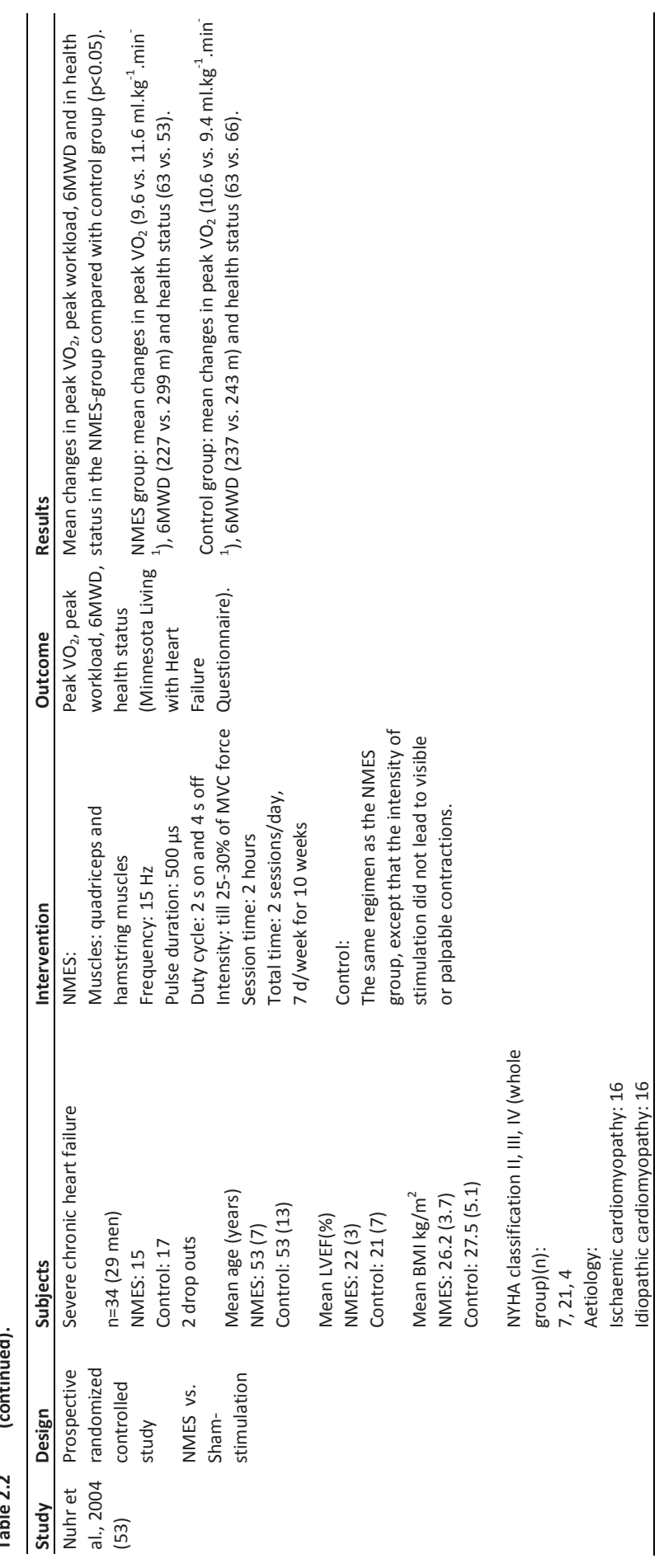




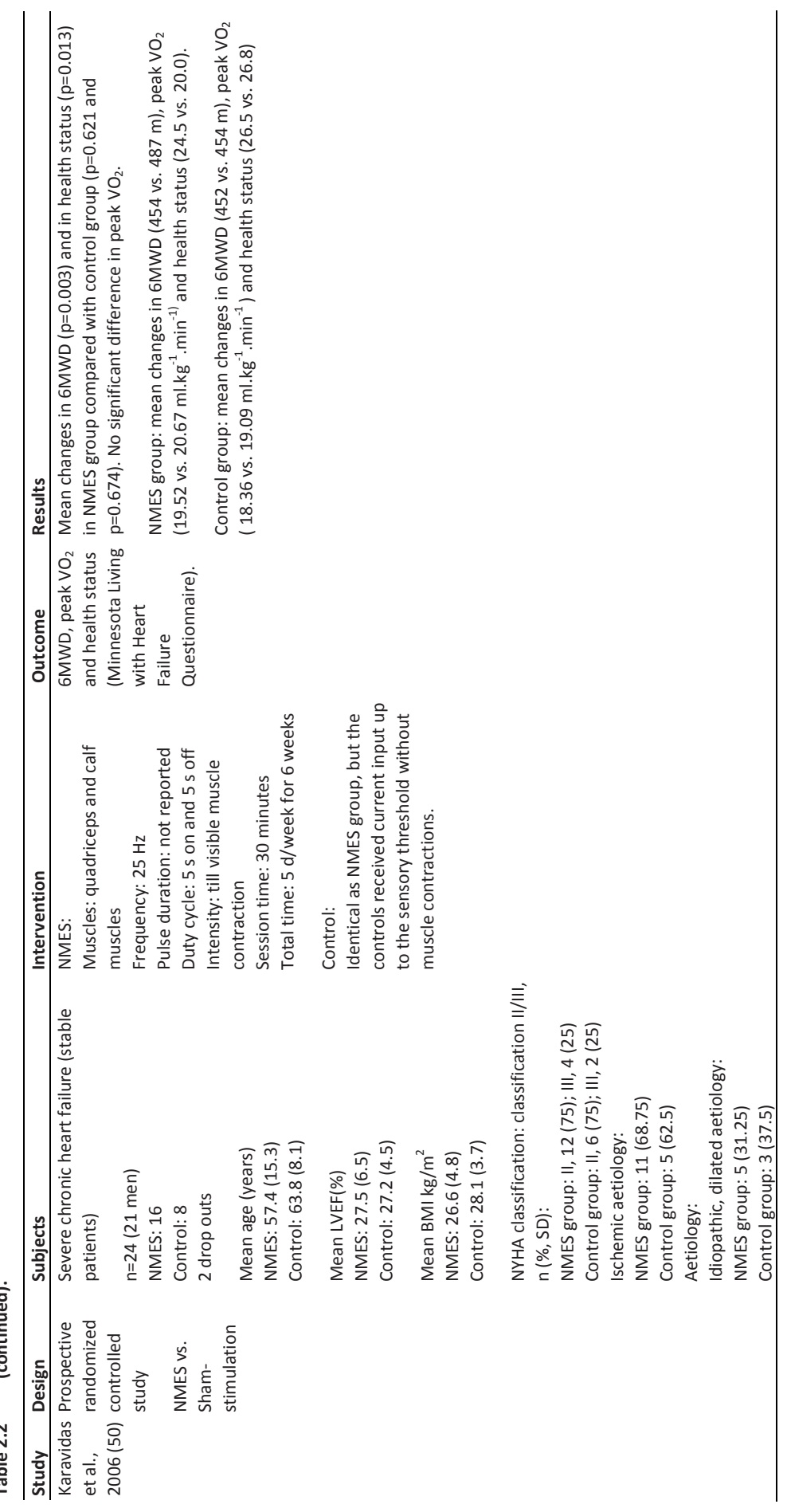




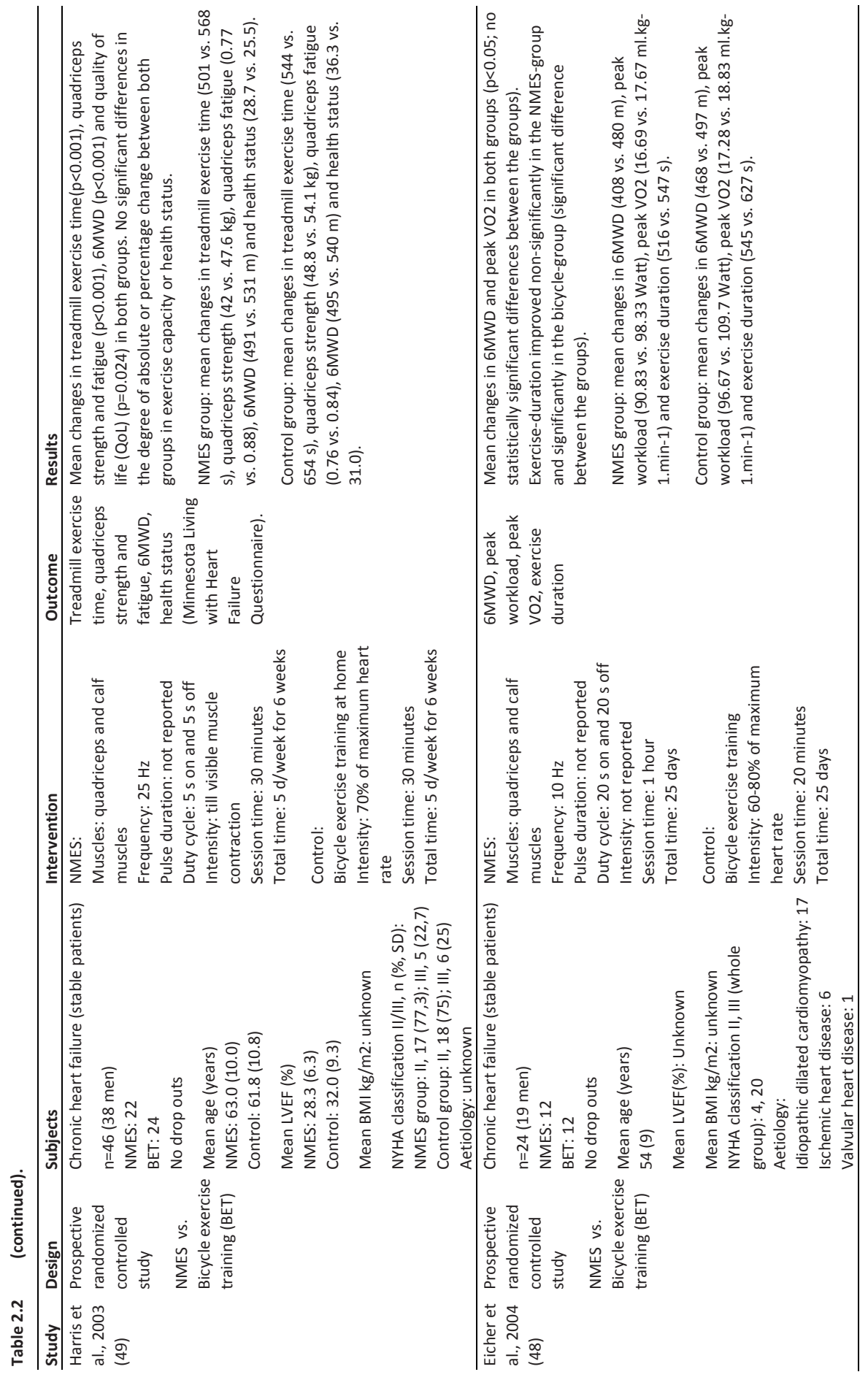




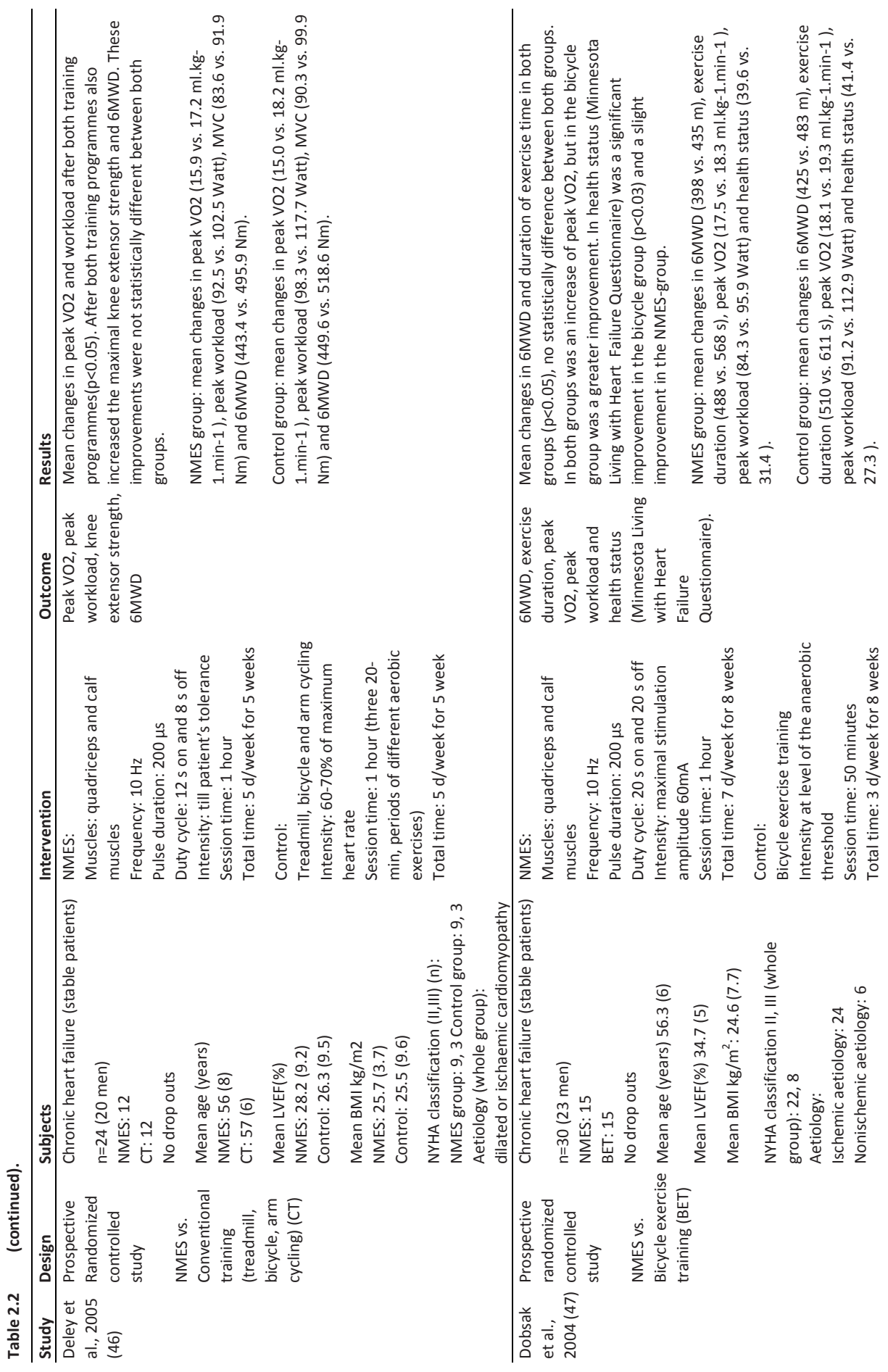




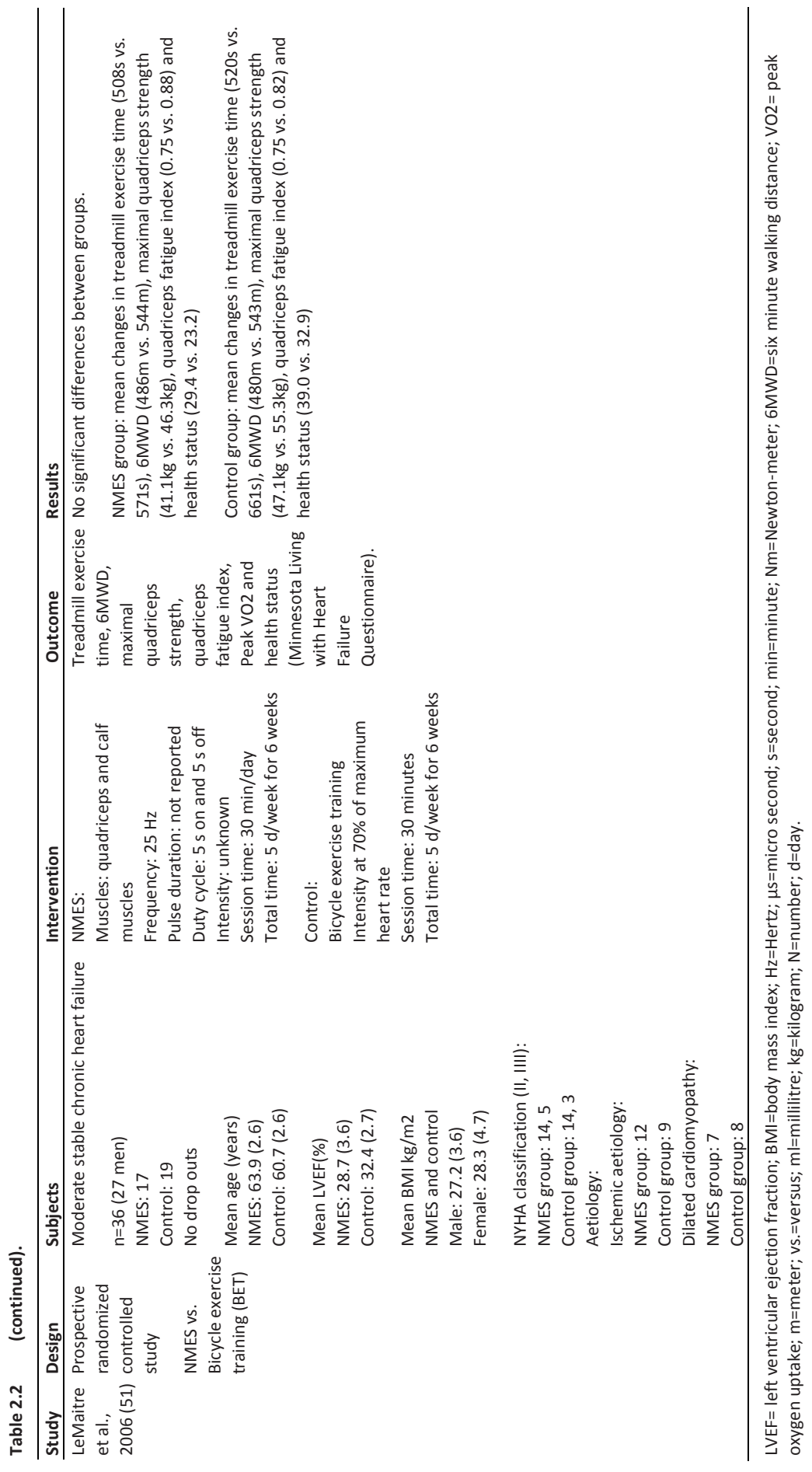




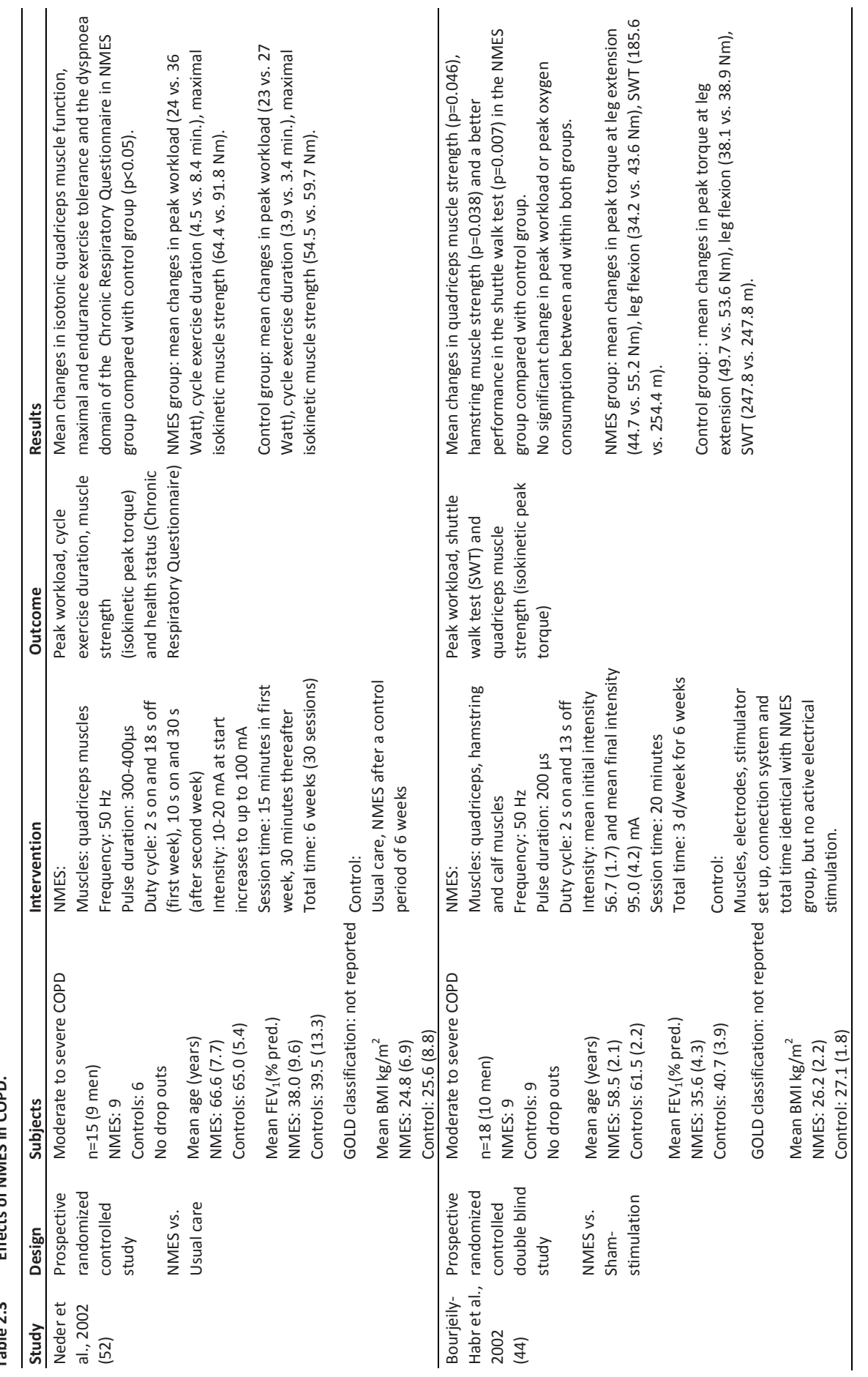




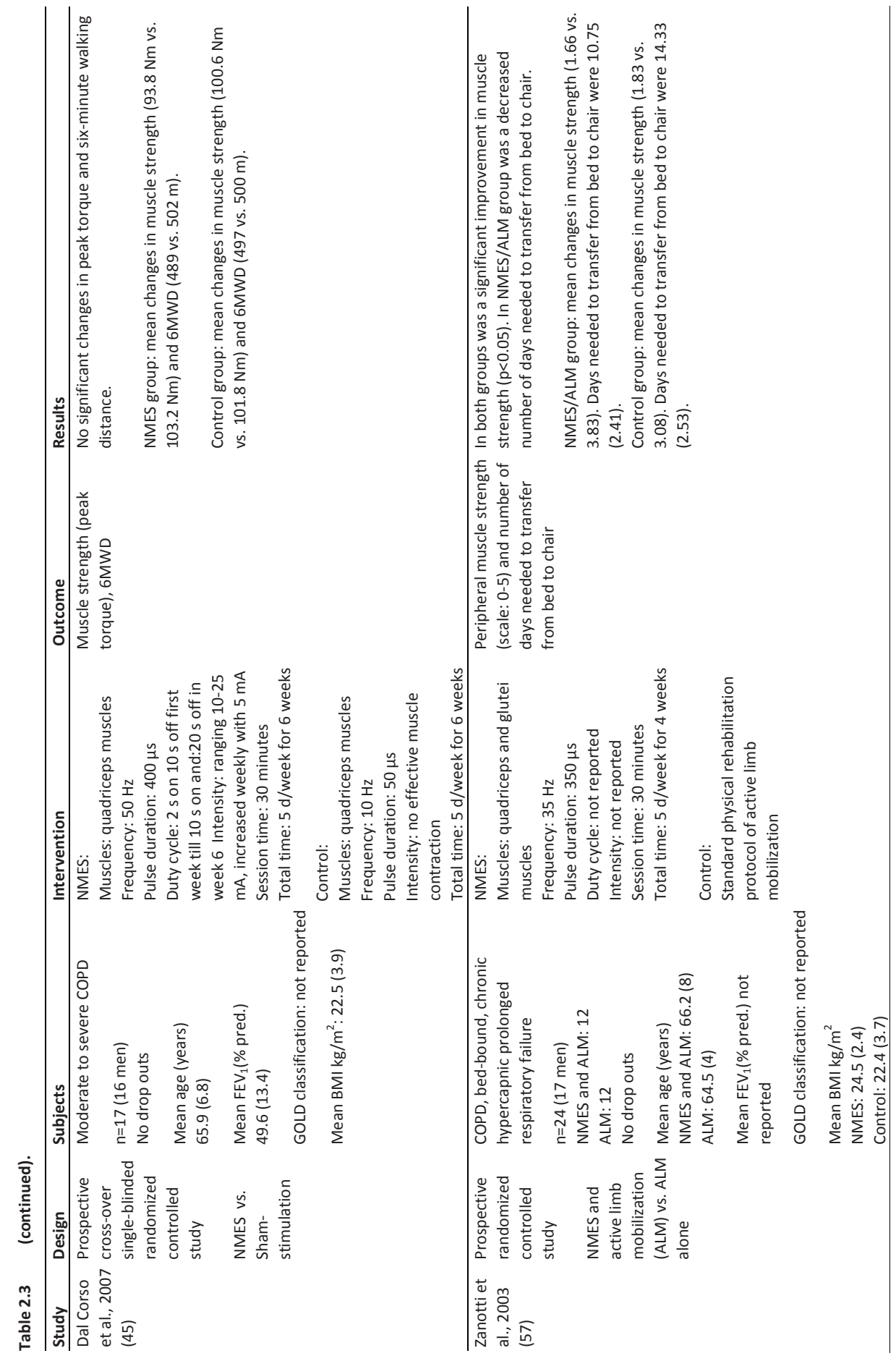




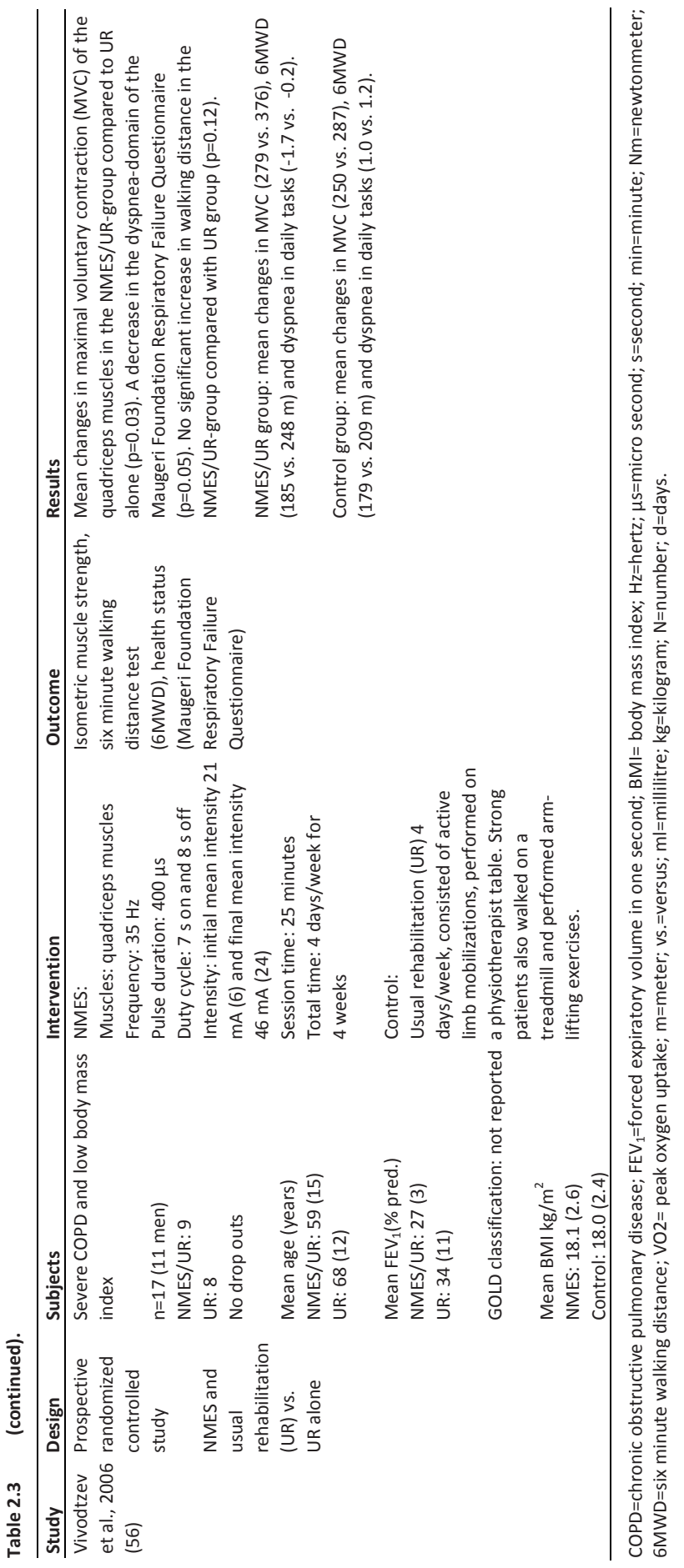




\section{NMES PROTOCOLS}

Different muscles of the lower extremities were stimulated in the identified trials: quadriceps femoris muscles $(44,45,55,56)$, quadriceps femoris muscles and hamstrings $(53,54)$, quadriceps femoris muscles and calf muscles (46-51), quadriceps femoris muscles and gluteus muscles (57), and quadriceps femoris muscles, hamstrings, and calf muscles (44).

All trials used biphasic impulse current forms ranging from 10 to 50 Hertz. Pulse duration, if reported, ranged between 200 and $700 \mu \mathrm{s}$. Duty cycle, if reported ranged between 2 seconds on, 4 seconds off and 10 seconds on, 50 seconds off. The intensity was increased until a visible strong muscle contraction or to the maximum level of toleration. Session time varied between 20 and 120 minutes, 1 to 2 times a day, for 3 to 7 days a week. The total number of sessions were at least 24 and maximum 70 (Tables 2.2, 2.3).

\section{EFFECTS OF NMES IN CHF}

\section{NMES versus usual care controls (Two Studies)}

Vaquero and colleagues reported significant increases in peak workload and peak $\mathrm{VO}_{2}$ in the NMES group (55). Quittan and colleagues (54) reported significant improvements in muscle strength, decrease of fatigue and a significant increase in health status on the Short Form 36 questionnaire, except on 'physical role'.

\section{NMES versus sham-stimulation (Two Studies)}

In both of these studies a significant improvement in health status was seen $(50,53)$. Also significant improvements in peak workload and peak aerobic capacity $\left(\mathrm{VO}_{2}\right)$ were reported (53), whereas the study by Karavidas and colleagues reported a significant improvement in six-minute walking distance (6MWD) (50).

\section{NMES versus endurance training (Five Studies)}

In all five of these studies non-significant improvements in $6 \mathrm{MWD}$, peak $\mathrm{VO}_{2}$, peak workload, exercise duration, muscle strength, and health status were reported between NMES and endurance training $(46-49,51)$. However, three studies scored $<6$ points on the PEDro scale (47-49).

\section{EFFECTS OF NMES IN COPD}

\section{NMES versus usual care controls (One Study)}

In this one study, (52) NMES resulted in significant improvements in quadriceps muscle function, exercise tolerance and health status in the NMES group (Table 2.3). 


\section{NMES versus sham stimulation (Two Studies)}

In these two studies the results are conflicting $(44,45)$. In the study by Dal Corso and colleagues no significant changes was found between NMES and sham stimulation (45). On the other hand Zanotti and colleagues reported significant improvements in quadriceps muscle strength and better performance in the shuttle walk test in the NMES group (57). Both studies scored 6 points on the PEDro scale.

NMES combined with active limb mobilization versus active limb mobilization alone (One Study)

In this study a significant improvement in muscle strength was seen in both treatment groups. The combined group needed a decreased number of days to transfer from bed to chair (57).

\section{NMES combined with usual rehabilitation versus usual rehabilitation alone (One Study)}

Significant improvement in maximal voluntary contraction of the quadriceps muscles along with a decrease in the dyspnea domain of the Maugeri Foundation Respiratory Failure Questionnaire was found when NMES was combined with rehabilitation compared to rehabilitation alone. However, there was no significant increase in walking distance in the combined group (56).

\section{SAFETY}

Safety was not a predefined primary outcome in the identified trials. Nevertheless, none of the trials reported (serious) adverse events, like injuries, hospital admission or death. In three of the nine CHF trials two to nine patients did not complete the trial $(50,53,54)$, but all patients with COPD did. In one CHF trial (55) and in one COPD trial (44), patients complained of mild self-limited muscle cramps or muscle soreness, which were related to NMES of the muscles of ambulation. Additionally, Neder and colleagues reported that severely disabled patients with COPD were able to continue home-based NMES during an acute exacerbation of the disease (52). In fact, COPD patients who were on a mechanical ventilator for $>30$ days benefited from NMES (57).

\section{DISCUSSION}

Although the limited number of studies and the heterogeneity in study design prohibit the use of meta-analysis, we can draw some conclusions from this systematic review. First, on balance most of the studies reported positive effects on skeletal muscle function, exercise capacity, and disease-specific health status in patients with CHF or 
COPD compared to a non-exercising control group. These findings are in line with a previous systematic review on the use of NMES for sports injuries (34). The one study that did not demonstrate significant improvements in muscle strength in COPD patients by Dal Corso and colleagues (45), did demonstrate changes in fiber types; a finding that is consistent with previously reported morphological changes in spinal cord injured patients (35). Second, in comparison with whole-body endurance training, NMES seems to be as effective in patients with CHF. Third, NMES of the muscles of ambulation appears to be safe in patients with moderate-to-very severe CHF or COPD. Indeed, none of authors reported serious adverse events. Nevertheless, physicians and physiotherapists have to be aware of the fact that none of the CHF patients were reported to have a cardiac pacemaker or an implantable cardioverter defibrillator. Nevertheless, it may be speculated that NMES may also be possible in these patients. Indeed, pilot studies have been shown that NMES of the muscles of ambulation is a safe treatment modality in patients with a cardiac pacemaker (58) or an implantable cardioverter defibrillator (59). NMES even appears to be possible during acute COPD exacerbations, in the patients' home environment (52) and in hypercapnic COPD patients who are prolonged bed-bound during mechanical ventilation (57). In fact, NMES has recently been proposed as a rehabilitation tool in highly-deconditioned patients with CHF (60).

Only full articles that were written in the English language were eligible for the present systematic review. The exclusion of congress abstracts from systematic analyses may have resulted in an overestimation of the effects of NMES in patients with CHF or COPD (61). Then again, there is no clear evidence that language restricted systematic analyses lead to biased estimates of intervention effectiveness (62).

\section{EFFECTS OF NMES IN CHF PATIENTS}

On average, in CHF positive statistically significant effects in muscle strength, exercise capacity, and disease-specific health status were found in the NMES groups compared with the control groups who underwent usual care (Table 2.2). Similar findings were reported after comparison with a sham-stimulation. This rules out an attention-placebo effect, and, in turn, points towards changes based on physiological adaptations in the skeletal muscles. Indeed, the mean improvement in 6MWD following NMES (+52 m) may even be of clinical relevance (63). Unfortunately, NMES trials in which patients underwent muscle biopsies are currently lacking in CHF patients. However, in patients with CHF, NMES improves endothelial function and also has anti-inflammatory effects (50).

Interestingly, in the trials in which the control group underwent aerobic exercise training $(46,49,51)$, non-significant differences in the mean change in exercise capacity were found between groups. These findings are in line with a systematic review concerning the effects of NMES in athletes following knee immobilization after ligament reconstruction surgery or injury (34). 


\section{EFFECTS OF NMES IN COPD PATIENTS}

On average, in patients with COPD positive, statistically significant effects in muscle strength, exercise capacity, and health status were also noted after NMES application. Three studies in COPD patients showed a significant difference in the mean change in muscle strength between NMES and control groups $(44,52,56)$. In one study, nonsignificant differences in the mean change in muscle strength between the groups was reported, but there was an improvement within the NMES group (57). One study showed no statistically significant positive effects on muscle strength (45). However, this study showed an increase in type II muscle fibers and a decrease in type I muscle fibers. These changes were not translated into an increased volitional strength. This is in line with the study of Martin et al (35), showing an increase in the oxidative capacity and endurance properties in spinal cord injuries.

The improvements following NMES seem to be clinically relevant. Indeed, the mean difference in change in incremental shuttle walk test distance between NMES and sham-stimulation was $68.8 \mathrm{~m}$, which clearly exceeds the minimal clinical important difference of $47 \mathrm{~m}$ (64). Moreover, the mean difference in change in the Chronic Repiratory Dyspnea Questionnaire domain "dyspnea” of 1.2 points per question also exceeded the minimal clinical important difference of 0.5 points per question (65). Finally, mechanically ventilated COPD patients who were bed bound for a prolonged duration were able to make an earlier transfer from bed to chair following NMES in combination with limb mobilization compared to limb mobilization only (57).

\section{NMES PROTOCOLS}

NMES protocols varied tremendously between the included trials. Pulse duration, if reported, differs between 200 and $700 \mu \mathrm{s}$. A pulse duration of 300-400 $\mu \mathrm{s}$ is recommended for large muscle groups, such as the quadriceps muscles and calf muscles (66). Overall, the CHF trials applied on average a lower frequency than the COPD trials. Variation frequency can affect the response to NMES as changes in skeletal muscle phenotype are dependent on frequency of stimulation. High stimulation frequencies ( $\geq 50 \mathrm{Hertz}$ ) seem to improve skeletal muscle strength $(32,67)$, whereas both low $(<50$ Hertz) and high stimulation frequencies improve oxidative capacity $(67,68)$. This difference is, however, not clear-cut because Perez and colleagues found in healthy young men an improvement in oxygen uptake kinetics and work efficiency with 6 weeks of stimulation with a frequency of 45-60 Hertz (69). They also showed that oxidative capacity and capillarization of type II fibers specifically improved. They used a range of duty cycles between 1:1 to $1: 5$. However, it is not known which duty cycle is optimal for effective treatment while avoiding excessive fatigue. In a study of wrist extensor muscle stimulation in patients with hemiparesis, duty-cycle ratios of $1: 1,1: 3$, and $1: 5$ were tested while monitoring muscle tension on repeat stimulation as a measure of fatigue (70). The 1:5 ratio was shown to produce the least amount of fatigue (70). While throughout the literature there are a wide variety of protocols used, there seems to be at least some agreement on the use of biphasic 
symmetrical pulses that last between 0.1 and $0.5 \mathrm{~ms}$ and are delivered at a pulse rate of 10 to 100 Hertz. Such pulses are widely accepted as being well tolerated.

This lack of protocol uniformity is likely a reflection of the mechanistic complexity of muscle contraction and strength gain. For example, normal daily life muscle contractions are different from muscle contractions induced by NMES. In natural movements, different motor units are stimulated in a hierarchical order to activate more motor units to make the contractions stronger. In contrast, during NMES this hierarchy is disrupted and all motor units are stimulated simultaneously (71). Furthermore, an electrical current that is applied externally will take the pathway of least resistance and, in turn, will recruit more type II fibers, which have a larger diameter, than type I fibers, which are constituted of smaller motoneurons with a higher resistance (71). The higher metabolic demand observed during NMES, as evidenced by reduced $\mathrm{pH}$ and more depletion of phosphocreatin, is in accordance with a preferential recruitment of type II fibers (72). This may be of particular interest for patients with CHF and COPD, who are known to have an atrophy of type II skeletal muscle fibers $(73,74)$.

Based on the results of the present systematic review, it seems appropriate to recommend that future trials be large RCTs of at least 6 weeks duration using concealed allocation, blinded therapists, and blinded outcome assessors. Efforts should be made to quantify the short-term and long-term effects of daily NMES of muscles of ambulation in patients with CHF or COPD. Additionally, inclusion criteria have to be well defined and the studies should focus on patients with CHF or COPD who experience overt dyspnea during conventional exercise training modalities (e.g., NYHA III/IV for CHF patients (75) and grade 4 to 5 on the Medical Research Council dyspnea scale for COPD patients (11). Remarkably, very few CHF patients with NYHA IV have been studied to date (table 2). It may also be of interest to look at patients who have an explicit loss of lean muscle mass $(13,76)$, although for these patients NMES may have to be combined with nutritional modulation (77). Further attention also needs to be given to optimal NMES protocols, because there is little consistency from study to study (Tables $2.2,2.3)$. RCTs on the effects of low-frequency versus high-frequency NMES in CHF and COPD patients who underwent concurrent muscle biopsies are also needed. It may also be worthwhile to study other large lower extremity muscle groups as to date only NMES on the quadriceps femoris has been shown to be effective in CHF and COPD $(44,55,56)$. Finally, all future studies should report changes in NMES pulse intensity over time in order to facilitate comparison between studies and to facilitate further development of NMES.

In conclusion, NMES holds out promise as a useful training modality within cardiopulmonary rehabilitative medicine that can be particularly useful for severely disabled patients with CHF or COPD. Indeed, it may even be feasible for home use and in patients who are not clinically stable. Nonetheless, larger well-designed trials are needed in order to improve our understanding of NMES and to clarify how NMES is to be optimally utilized. 


\section{REFERENCES}

1. Lopez AD, Mathers CD, Ezzati M, Jamison DT, Murray CJ. Global and regional burden of disease and risk factors, 2001: systematic analysis of population health data. Lancet. 2006;367(9524):1747-57.

2. Mathers CD, Loncar D. Projections of global mortality and burden of disease from 2002 to 2030. PLoS Med. 2006;3(11):e442.

3. Murray CJ, Lopez AD. Mortality by cause for eight regions of the world: Global Burden of Disease Study. Lancet. 1997;349(9061):1269-76.

4. Swedberg K, Cleland J, Dargie H, Drexler H, Follath F, Komajda M, et al. Guidelines for the diagnosis and treatment of chronic heart failure: executive summary (update 2005): The Task Force for the Diagnosis and Treatment of Chronic Heart Failure of the European Society of Cardiology. Eur Heart J. 2005;26(11):1115-40.

5. Pauwels RA, Buist AS, Calverley PM, Jenkins CR, Hurd SS. Global strategy for the diagnosis, management, and prevention of chronic obstructive pulmonary disease. NHLBI/WHO Global Initiative for Chronic Obstructive Lung Disease (GOLD) Workshop summary. Am J Respir Crit Care Med. 2001;163(5):1256-76.

6. Davis RC, Hobbs FD, Lip GY. ABC of heart failure. History and epidemiology. BMJ. 2000;320(7226):39-42.

7. Celli BR, MacNee W. Standards for the diagnosis and treatment of patients with COPD: a summary of the ATS/ERS position paper. Eur Respir J. 2004;23(6):932-46.

8. Janssen DJ, Spruit MA, Wouters EF, Schols JM. Daily symptom burden in end-stage chronic organ failure: a systematic review. Palliat Med. 2008;22(8):938-48.

9. Connors AF, Jr., Dawson NV, Thomas C, Harrell FE, Jr., Desbiens N, Fulkerson WJ, et al. Outcomes following acute exacerbation of severe chronic obstructive lung disease. The SUPPORT investigators (Study to Understand Prognoses and Preferences for Outcomes and Risks of Treatments). Am J Respir Crit Care Med. 1996;154(4 Pt 1):959-67.

10. Lee DS, Austin PC, Rouleau JL, Liu PP, Naimark D, Tu JV. Predicting mortality among patients hospitalized for heart failure: derivation and validation of a clinical model. JAMA. 2003;290(19):2581-7.

11. Spruit MA, Pennings HJ, Janssen PP, Does JD, Scroyen S, Akkermans MA, et al. Extra-pulmonary features in COPD patients entering rehabilitation after stratification for MRC dyspnea grade. Respir Med. 2007;101(12):2454-63.

12. Walke LM, Byers AL, Tinetti ME, Dubin JA, McCorkle R, Fried TR. Range and severity of symptoms over time among older adults with chronic obstructive pulmonary disease and heart failure. Arch Intern Med. 2007;167(22):2503-8.

13. Gosker HR, Lencer NH, Franssen FM, van der Vusse GJ, Wouters EF, Schols AM. Striking similarities in systemic factors contributing to decreased exercise capacity in patients with severe chronic heart failure or COPD. Chest. 2003;123(5):1416-24.

14. Anker SD, Ponikowski PP, Clark AL, Leyva F, Rauchhaus M, Kemp M, et al. Cytokines and neurohormones relating to body composition alterations in the wasting syndrome of chronic heart failure. Eur Heart J. 1999;20(9):683-93.

15. Schols AM, Buurman WA, Staal van den Brekel AJ, Dentener MA, Wouters EF. Evidence for a relation between metabolic derangements and increased levels of inflammatory mediators in a subgroup of patients with chronic obstructive pulmonary disease. Thorax. 1996;51(8):819-24.

16. Anker SD, Swan JW, Volterrani M, Chua TP, Clark AL, Poole-Wilson PA, et al. The influence of muscle mass, strength, fatigability and blood flow on exercise capacity in cachectic and non-cachectic patients with chronic heart failure. Eur Heart J. 1997;18(2):259-69.

17. Bernard S, LeBlanc P, Whittom F, Carrier G, Jobin J, Belleau R, et al. Peripheral muscle weakness in patients with chronic obstructive pulmonary disease. Am J Respir Crit Care Med. 1998;158(2):629-34.

18. Broekhuizen R, Grimble RF, Howell WM, Shale DJ, Creutzberg EC, Wouters EF, et al. Pulmonary cachexia, systemic inflammatory profile, and the interleukin 1 beta -511 single nucleotide polymorphism. Am J Clin Nutr. 2005;82(5):1059-64.

19. Clark A, Rafferty D, Arbuthnott K. Relationship between isokinetic muscle strength and exercise capacity in chronic heart failure. Int J Cardiol. 1997;59(2):145-8. 
20. Gosselink R, Troosters T, Decramer M. Peripheral muscle weakness contributes to exercise limitation in COPD. Am J Respir Crit Care Med. 1996;153(3):976-80.

21. Hulsmann M, Quittan M, Berger R, Crevenna R, Springer C, Nuhr M, et al. Muscle strength as a predictor of long-term survival in severe congestive heart failure. Eur J Heart Fail. 2004;6(1):101-7.

22. Mostert R, Goris A, Weling-Scheepers C, Wouters EF, Schols AM. Tissue depletion and health related quality of life in patients with chronic obstructive pulmonary disease. Respir Med. 2000;94(9):859-67.

23. Rutten EP, Franssen FM, Engelen MP, Wouters EF, Deutz NE, Schols AM. Greater whole-body myofibrillar protein breakdown in cachectic patients with chronic obstructive pulmonary disease. Am J Clin Nutr. 2006;83(4):829-34.

24. Pitta F, Troosters T, Spruit MA, Probst VS, Decramer M, Gosselink R. Characteristics of physical activities in daily life in chronic obstructive pulmonary disease. Am J Respir Crit Care Med. 2005;171(9):972-7.

25. van den Berg-Emons RJ, Bussmann JB, Balk AH, Stam HJ. Factors associated with the level of movement-related everyday activity and quality of life in people with chronic heart failure. Phys Ther. 2005;85(12):1340-8.

26. Pitta F, Troosters T, Probst VS, Lucas S, Decramer M, Gosselink R. Potential consequences for stable chronic obstructive pulmonary disease patients who do not get the recommended minimum daily amount of physical activity. J Bras Pneumol. 2006;32(4):301-8.

27. Rees K, Taylor RS, Singh S, Coats AJ, Ebrahim S. Exercise based rehabilitation for heart failure. Cochrane Database Syst Rev. 2004(3):CD003331.

28. Spruit MA, Vanderhoven-Augustin I, Janssen PP, Wouters EF. Integration of pulmonary rehabilitation in COPD. Lancet. 2008;371(9606):12-3.

29. Lacasse Y, Goldstein R, Lasserson TJ, Martin S. Pulmonary rehabilitation for chronic obstructive pulmonary disease. Cochrane Database Syst Rev. 2006(4):CD003793.

30. Maltais F, LeBlanc P, Jobin J, Berube C, Bruneau J, Carrier L, et al. Intensity of training and physiologic adaptation in patients with chronic obstructive pulmonary disease. Am J Respir Crit Care Med. 1997;155(2):555-61.

31. Spruit MA, Wouters EF. New modalities of pulmonary rehabilitation in patients with chronic obstructive pulmonary disease. Sports Med. 2007;37(6):501-18.

32. Vanderthommen M, Crielaard JM. [Muscle electric stimulation in sports medicine]. Rev Med Liege. 2001;56(5):391-5. Epub 2001/07/31. Electromyostimulation en medecine du sport.

33. Vivodtzev I, Lacasse $Y$, Maltais F. Neuromuscular electrical stimulation of the lower limbs in patients with chronic obstructive pulmonary disease. J Cardiopulm Rehabil Prev. 2008;28(2):79-91.

34. Lake DA. Neuromuscular electrical stimulation. An overview and its application in the treatment of sports injuries. Sports Med. 1992;13(5):320-36.

35. Martin TP, Stein RB, Hoeppner PH, Reid DC. Influence of electrical stimulation on the morphological and metabolic properties of paralyzed muscle. J Appl Physiol. 1992;72(4):1401-6.

36. Theriault R, Boulay MR, Theriault G, Simoneau JA. Electrical stimulation-induced changes in performance and fiber type proportion of human knee extensor muscles. Eur J Appl Physiol Occup Physiol. 1996;74(4):311-7.

37. Sillen MJ, Janssen PP, Akkermans MA, Wouters EF, Spruit MA. The metabolic response during resistance training and neuromuscular electrical stimulation (NMES) in patients with COPD, a pilot study. Respir Med. 2008;102(5):786-9.

38. Bausewein C, Booth S, Gysels M, Higginson I. Non-pharmacological interventions for breathlessness in advanced stages of malignant and non-malignant diseases. Cochrane Database Syst Rev. 2008(2):CD005623.

39. Rabe KF, Hurd S, Anzueto A, Barnes PJ, Buist SA, Calverley P, et al. Global strategy for the diagnosis, management, and prevention of chronic obstructive pulmonary disease: GOLD executive summary. Am J Respir Crit Care Med. 2007;176(6):532-55.

40. Criteria Committee of the New York Heart Association. Nomenclature and criteria for diagnosis of diseases of the heart and great vessels. 9th ed Boston, MA: Little, Brown. 1994:253-6.

41. Sherrington C, Herbert RD, Maher CG, Moseley AM. PEDro. A database of randomized trials and systematic reviews in physiotherapy. Man Ther. 2000;5(4):223-6.

42. Brennan P, Silman A. Statistical methods for assessing observer variability in clinical measures. BMJ. 1992;304(6840):1491-4. 
43. Foley NC, Bhogal SK, Teasell RW, Bureau Y, Speechley MR. Estimates of quality and reliability with the physiotherapy evidence-based database scale to assess the methodology of randomized controlled trials of pharmacological and nonpharmacological interventions. Phys Ther. 2006;86(6):817-24.

44. Bourjeily-Habr G, Rochester CL, Palermo F, Snyder P, Mohsenin V. Randomised controlled trial of transcutaneous electrical muscle stimulation of the lower extremities in patients with chronic obstructive pulmonary disease. Thorax. 2002;57(12):1045-9.

45. Dal Corso S, Napolis L, Malaguti C, Gimenes AC, Albuquerque A, Nogueira CR, et al. Skeletal muscle structure and function in response to electrical stimulation in moderately impaired COPD patients. Respir Med. 2007;101(6):1236-43.

46. Deley G, Kervio G, Verges B, Hannequin A, Petitdant MF, Salmi-Belmihoub S, et al. Comparison of lowfrequency electrical myostimulation and conventional aerobic exercise training in patients with chronic heart failure. Eur J Cardiovasc Prev Rehabil. 2005;12(3):226-33.

47. Dobsak P, Novakova M, Fiser B, Siegelova J, Balcarkova P, Spinarova L, et al. Electrical stimulation of skeletal muscles. An alternative to aerobic exercise training in patients with chronic heart failure? Int Heart J. 2006;47(3):441-53.

48. Eicher JC, Dobsak P, Berteau O, Walker P, Verges B, Maillefert JF, et al. Rehabilitation in chronic congestive heart failure: comparison of bicycle training and muscle electrical stimulation. Scr Med (Brno). 2004;77:261-70.

49. Harris S, LeMaitre JP, Mackenzie G, Fox KA, Denvir MA. A randomised study of home-based electrical stimulation of the legs and conventional bicycle exercise training for patients with chronic heart failure. Eur Heart J. 2003;24(9):871-8.

50. Karavidas Al, Raisakis KG, Parissis JT, Tsekoura DK, Adamopoulos S, Korres DA, et al. Functional electrical stimulation improves endothelial function and reduces peripheral immune responses in patients with chronic heart failure. Eur J Cardiovasc Prev Rehabil. 2006;13(4):592-7.

51. LeMaitre JP, Harris S, Hannan J, Fox KA, Denvir MA. Maximum oxygen uptake corrected for skeletal muscle mass accurately predicts functional improvements following exercise training in chronic heart failure. Eur J Heart Fail. 2006;8(3):243-8.

52. Neder JA, Sword D, Ward SA, Mackay E, Cochrane LM, Clark CJ. Home based neuromuscular electrical stimulation as a new rehabilitative strategy for severely disabled patients with chronic obstructive pulmonary disease (COPD). Thorax. 2002;57(4):333-7.

53. Nuhr MJ, Pette D, Berger R, Quittan M, Crevenna R, Huelsman M, et al. Beneficial effects of chronic low-frequency stimulation of thigh muscles in patients with advanced chronic heart failure. Eur Heart J. 2004;25(2):136-43.

54. Quittan M, Wiesinger GF, Sturm B, Puig S, Mayr W, Sochor A, et al. Improvement of thigh muscles by neuromuscular electrical stimulation in patients with refractory heart failure: a single-blind, randomized, controlled trial. Am J Phys Med Rehabil. 2001;80(3):206-14; quiz 15-6, 24.

55. Vaquero AF, Chicharro JL, Gil L, Ruiz MP, Sanchez V, Lucia A, et al. Effects of muscle electrical stimulation on peak VO2 in cardiac transplant patients. Int J Sports Med. 1998;19(5):317-22.

56. Vivodtzev I, Pepin JL, Vottero G, Mayer V, Porsin B, Levy P, et al. Improvement in quadriceps strength and dyspnea in daily tasks after 1 month of electrical stimulation in severely deconditioned and malnourished COPD. Chest. 2006;129(6):1540-8.

57. Zanotti E, Felicetti G, Maini M, Fracchia C. Peripheral muscle strength training in bed-bound patients with COPD receiving mechanical ventilation: effect of electrical stimulation. Chest. 2003;124(1):292-6.

58. Crevenna R, Mayr W, Keilani M, Pleiner J, Nuhr M, Quittan M, et al. Safety of a combined strength and endurance training using neuromuscular electrical stimulation of thigh muscles in patients with heart failure and bipolar sensing cardiac pacemakers. Wien Klin Wochenschr. 2003;115(19-20):710-4.

59. Crevenna R, Wolzt M, Fialka-Moser V, Keilani M, Nuhr M, Paternostro-Sluga T, et al. Long-term transcutaneous neuromuscular electrical stimulation in patients with bipolar sensing implantable cardioverter defibrillators: a pilot safety study. Artif Organs. 2004;28(1):99-102.

60. Deley G, Kervio G, Verges B, Hannequin A, Petitdant MF, Grassi B, et al. Neuromuscular adaptations to low-frequency stimulation training in a patient with chronic heart failure. Am J Phys Med Rehabil. 2008;87(6):502-9.

61. McAuley L, Pham B, Tugwell P, Moher D. Does the inclusion of grey literature influence estimates of intervention effectiveness reported in meta-analyses? Lancet. 2000;356(9237):1228-31. 
62. Moher D, Pham B, Klassen TP, Schulz KF, Berlin JA, Jadad AR, et al. What contributions do languages other than English make on the results of meta-analyses? J Clin Epidemiol. 2000;53(9):964-72.

63. Puhan MA, Mador MJ, Held U, Goldstein R, Guyatt GH, Schunemann HJ. Interpretation of treatment changes in 6-minute walk distance in patients with COPD. Eur Respir J. 2008;32(3):637-43.

64. Singh SJ, Jones PW, Evans R, Morgan MD. Minimum clinically important improvement for the incremental shuttle walking test. Thorax. 2008;63(9):775-7.

65. Jaeschke R, Singer J, Guyatt GH. Measurement of health status. Ascertaining the minimal clinically important difference. Control Clin Trials. 1989;10(4):407-15.

66. Bowman BR, Baker LL. Effects of waveform parameters on comfort during transcutaneous neuromuscular electrical stimulation. Ann Biomed Eng. 1985;13(1):59-74.

67. Perez M, Lucia A, Rivero JL, Serrano AL, Calbet JA, Delgado MA, et al. Effects of transcutaneous shortterm electrical stimulation on $\mathrm{M}$. vastus lateralis characteristics of healthy young men. Pflugers Arch. 2002;443(5-6):866-74.

68. Cabric M, Appell HJ. Effect of electrical stimulation of high and low frequency on maximum isometric force and some morphological characteristics in men. Int J Sports Med. 1987;8(4):256-60.

69. Perez M, Lucia A, Santalla A, Chicharro JL. Effects of electrical stimulation on VO2 kinetics and delta efficiency in healthy young men. Br J Sports Med. 2003;37(2):140-3.

70. Packman-Braun R. Relationship between functional electrical stimulation duty cycle and fatigue in wrist extensor muscles of patients with hemiparesis. Phys Ther. 1988;68(1):51-6.

71. Henneman E, Clamann HP, Gillies JD, Skinner RD. Rank order of motoneurons within a pool: law of combination. J Neurophysiol. 1974;37(6):1338-49.

72. Eriksson E, Haggmark T. Comparison of isometric muscle training and electrical stimulation supplementing isometric muscle training in the recovery after major knee ligament surgery. A preliminary report. Am J Sports Med. 1979;7(3):169-71.

73. Gosker HR, Engelen MP, van Mameren H, van Dijk PJ, van der Vusse GJ, Wouters EF, et al. Muscle fiber type IIX atrophy is involved in the loss of fat-free mass in chronic obstructive pulmonary disease. Am J Clin Nutr. 2002;76(1):113-9.

74. Larsen Al, Lindal S, Aukrust P, Toft I, Aarsland T, Dickstein K. Effect of exercise training on skeletal muscle fibre characteristics in men with chronic heart failure. Correlation between skeletal muscle alterations, cytokines and exercise capacity. Int J Cardiol. 2002;83(1):25-32.

75. Raphael C, Briscoe C, Davies J, Ian Whinnett Z, Manisty C, Sutton R, et al. Limitations of the New York Heart Association functional classification system and self-reported walking distances in chronic heart failure. Heart. 2007;93(4):476-82.

76. Uszko-Lencer NH, Bothmer F, van Pol PE, Schols AM. Measuring body composition in chronic heart failure: a comparison of methods. Eur J Heart Fail. 2006;8(2):208-14.

77. Anker SD, John M, Pedersen PU, Raguso C, Cicoira M, Dardai E, et al. ESPEN Guidelines on Enteral Nutrition: Cardiology and pulmonology. Clin Nutr. 2006;25(2):311-8. 


\section{APPENDIX 2.1}

\section{SeARCH STRATEgY (JANUARY 12, 2007)}

\section{Medline (1966 to 2007)}

\# 1 ("Pulmonary Disease, Chronic Obstructive" [mh] OR "COPD") AND ("Electric Stimulation" [mh] OR "Electric Stimulation Therapy" [mh] OR electric* [tw] AND neuromuscular [tw] AND electric* [tw] AND stimulat* [tw] OR functional[tw] AND electric* [tw] AND stimulat*[tw]) AND (randomized controlled trial [pt] OR controlled clinical trial [pt] OR randomized controlled trials [mh] OR random allocation [mh] OR double-blind method [mh] OR single-blind method [mh] OR clinical trial [pt] OR clinical trials [mh] OR "clinical trial" [tw] OR ((singl* [tw] OR doubl* [tw] OR trebl* [tw] OR tripl* [tw]) AND (mask* [tw] OR blind* [tw])) OR "latin square" [tw] OR placebos [mh] OR placebo* [tw] OR random* [tw] OR research design [mh:noexp] OR comparative study [mh] OR evaluation studies [mh] OR follow-up studies [mh] OR prospective studies [mh] OR cross-over studies [mh] OR control* [tw] OR prospectiv* [tw] OR volunteer* [tw]) NOT (animal [mh] NOT human [mh])

\#2 (Heart Failure, Congestive [mh] OR cardiac [tw] AND failure [tw] OR heart [tw] AND transplant [tw] OR cardiac [tw] AND transplant [tw]) AND (“Electric Stimulation" [mh] OR "Electric Stimulation Therapy" [mh] OR electric* [tw] AND neuromuscular [tw] AND electric* [tw] AND stimulat* [tw] OR functional[tw] AND electric* [tw] AND stimulat*[tw]) AND (randomized controlled trial [pt] OR controlled clinical trial [pt] OR randomized controlled trials [mh] OR random allocation [mh] OR double-blind method [mh] OR single-blind method [mh] OR clinical trial [pt] OR clinical trials [mh] OR "clinical trial" [tw] OR ((singl* [tw] OR doubl* [tw] OR trebl* [tw] OR tripl* [tw]) AND (mask* [tw] OR blind* [tw])) OR "latin square" [tw] OR placebos [mh] OR placebo* [tw] OR random* [tw] OR research design [mh:noexp] OR comparative study [mh] OR evaluation studies [mh] OR follow-up studies [mh] OR prospective studies [mh] OR cross-over studies [mh] OR control* [tw] OR prospectiv* [tw] OR volunteer* [tw]) NOT (animal [mh] NOT human [mh])

\section{Embase (1974 to 2007)}

\#1 (“Pulmonary Disease, Chronic Obstructive" [mh] OR “COPD”) AND (“Electric Stimulation" [mh] OR "Electric Stimulation Therapy" [mh] OR electric* [tw] AND neuromuscular [tw] AND electric* [tw] AND stimulat* [tw] OR functional[tw] AND electric* [tw] AND stimulat*[tw]) AND ([controlled clinical trial]/lim OR [randomized controlled trial]/lim AND [1966-2007]/py

\#2 (Heart Failure, Congestive [mh] OR cardiac [tw] AND failure [tw] OR heart [tw] AND transplant [tw] OR cardiac [tw] AND transplant [tw]) AND ("Electric 
Stimulation"[mh] OR "Electric Stimulation Therapy" [mh] OR electric* [tw] AND neuromuscular [tw] AND electric* [tw] AND stimulat* [tw] OR functional[tw] AND electric* [tw] AND stimulat*[tw]) AND ([controlled clinical trial]/lim OR [randomized controlled trial]/lim AND [1966-2007]/py

\section{Cochrane (1800 to -2007), CINAHL (1982 to 2007), and PEDro (1982 to 2007)}

\#1 pulmonary disease chronic obstructive AND electric* stimulat*

\#2 pulmonary disease chronic obstructive AND electric* stimulat* therapy

\#3 pulmonary disease chronic obstructive AND neuromuscular electric* stimulat*

\#4 pulmonary disease chronic obstructive AND functional electric* stimulat*

\#5 COPD AND electric* stimulat*

\#6 COPD AND electric* stimulat* therapy

\#7 COPD AND neuromuscular electric* stimulat*

\#8 COPD AND functional electric stimulat*

\#9 heart failure AND electric* stimulat*

\#10 cardiac failure AND electric* stimulat*

\#11 heart transplant AND electric* stimulat*

\#12 cardiac transplant AND electric* stimulat*

\#13 heart failure AND neuromuscular electric* stimulat*

\#14 cardiac failure AND neuromuscular electric* stimulat*

\#15 heart transplant AND neuromuscular electric* stimulat*

\#16 cardiac transplant AND neuromuscular electric* stimulat*

\#17 heart failure AND functional electric* stimulat*

\#18 cardiac failure AND functional electric* stimulat*

\#19 heart transplant AND functional electric* stimulat*

\#20 cardiac transplant AND functional electric* stimulat* 


\section{APPENDIX 2.2}

\section{PEDRo sCALE}

Each satisfied item (except the first item) contributes one point to the total PEDro score (range, 0 to 10 points).

1. Eligibility criteria were specified: yes/no.

2. Subjects were randomly allocated to groups (in a crossover study, subjects were randomly allocated an order in which treatments were received): yes/no.

3. Allocation was concealed: yes/no.

4. The groups were similar at baseline regarding the most important prognostic indicators: yes/no.

5. There was blinding of all subjects: yes/no.

6. There was blinding of all therapists who administered the therapy: yes/no.

7. There was blinding of all assessors who measured at least one key outcome: yes/no.

8. Measures of at least one key outcome were obtained from more than $85 \%$ of the subjects initially allocated to groups: yes/no

9. all subjects for whom outcome measures were available received the treatment or control condition as allocated or, where this was not the case, data for at least one key outcome was analysed by "intention to treat": yes/no.

10. The results of between-group statistical comparisons are reported for at least one key outcome: yes/no.

11. The study provides both point measures and measures of variability for at least one key outcome: yes/no. 


\section{CHAPTER 3}

Metabolic and structural changes in lower-limb skeletal muscle following neuromuscular electrical stimulation:

a systematic review

Maurice JH Sillen, Frits ME Franssen, Harry R Gosker, Emiel FM Wouters, Martijn A Spruit

PLoS One 2013;8:e69391 


\section{ABSTRACT}

\section{Background}

Transcutaneous neuromuscular electrical stimulation (NMES) can be applied as a complementary intervention to regular exercise training programs. A distinction can be made between high-frequency (HF) NMES and low-frequency (LF) NMES. In order to increase understanding of the mechanisms of functional improvements following NMES, the purpose of this study was to systematically review changes in enzyme activity, muscle fiber type composition and muscle fiber size in human lower-limb skeletal muscles following only NMES.

\section{Methods}

Trials were collected up to March 2012 and were identified by searching the Medline/PubMed, EMBASE, Cochrane Central Register of Controlled Trials, CINAHL and The Physical Therapy Evidence Database (PEDro) databases and reference lists. 18 trials were reviewed in detail: 8 trials studied changes in enzyme activities, 7 trials studied changes in muscle fiber type composition and 14 trials studied changes in muscle fiber size following NMES.

\section{Results}

The methodological quality generally was poor, and the heterogeneity in study design, study population, NMES features and outcome parameters prohibited the use of metaanalysis.

Most of the LF-NMES studies reported significant increases in oxidative enzyme activity, while the results concerning changes in muscle fiber composition and muscle size were conflicting. HF-NMES significantly increased muscle size in $55 \%$ of the studies.

\section{Conclusion}

NMES seems to be a training modality resulting in changes in oxidative enzyme activity, skeletal muscle fiber type and skeletal muscle fiber size. However, considering the small sample sizes, the variance in study populations, the non-randomized controlled study designs, the variance in primary outcomes, and the large heterogeneity in NMES protocols, it is difficult to draw definitive conclusions about the effects of stimulation frequencies on muscular changes. 


\section{INTRODUCTION}

Regular exercise training programs consist of a combination of aerobic and strengthening exercises for developing and maintaining muscular endurance and strength, respectively (1). Indeed, combined training modalities result in improvements in body composition and cardiorespiratory fitness. These improvements can partially be explained by intramuscular changes, such as an increased enzyme activity and an increased muscle fiber size $(2,3)$.

These intramuscular changes are dependent on the type of exercise training. Generally, aerobic exercise training results in increased levels of oxidative enzymes (4) and only a marginal increase in percentage type I fibers (5); whereas resistance training results in increased levels of glycolytic enzymes (6) and an increase in percentage and size of type II fibers (7-10).

Neuromuscular electrical stimulation (NMES) can be applied as a complement intervention to voluntary exercise training (11). NMES involves the application of an electric current through electrodes placed on the skin over the targeted muscles, thereby depolarizing motor endplates via the motor nerve and, in turn, inducing skeletal muscle contractions $(12,13)$. NMES is composed of stimulation-rest cycles situated in regard to muscle motor points (14). In contrast to voluntary muscle actions, NMES activates the muscle to a greater extent under identical technical conditions (15). At identical levels of workload (10\% of the quadriceps maximum isometric voluntary torque), the muscle reaches higher values in blood flow and oxygen consumption during NMES compared with voluntary contractions (15). Moreover, a single session of NMES is sufficient to stimulate molecular-level responses, which are indicative of the initiation of myogenic processes in skeletal muscle, while an additional NMES session (a total of 14 minutes spread over 2 days), was sufficient to induce an increase in the concentration of total ribonucleic acid (RNA) (16), most likely representing an increase in muscle protein synthesis. There is sufficient evidence that NMES induced contractions differ physiologically compared to voluntary contractions (17). In human studies contradictory findings on motor unit recruitment order have been found (18). Some studies suggest preferential or selective activation of fast motor units with NMES $(19,20)$, whereas others suggest that motor unit recruitment during NMES reflects a non-selective, spatially fixed, and temporally synchronous pattern rather than in a reversal of the physiological voluntary recruitment order (17). These diverse results could have been related to differences in protocols and stimulated muscles (14).

In daily clinical practice, lower-limb NMES improves skeletal muscle mass and function, exercise capacity and health status (21-23), particularly in subjects who are unable to perform or complete volitional exercise training programs. Therefore, NMES may be valuable in dyspneic and deconditioned patients with chronic organ failure due to the low metabolic load on the impaired cardio-respiratory system $(23,24)$. 
NMES training sessions generally last 10-30 minutes during a 4- to 5-week period that involves 20-25 sessions to increase peripheral muscle function (14). 2 types of NMES frequencies can be distinguished: high-frequency NMES (HF-NMES, $\geq 50$ Hertz); and low-frequency NMES (LF-NMES, $\leq 20$ Hertz) (13,14,25-27). Frequencies of 50 Hertz and above induce a fused tetanus $(28,29)$ and generate higher torques than low frequencies (30). The mechanisms by which NMES results in increased muscle strength or endurance are poorly understood. In isolated muscles in rats HF-NMES induces anabolic processes similar to resistance training (e.g. increased PKB-TSC2-mTor and protein synthesis) and LF-NMES similar to endurance training (AMPK-PG C1 $\alpha$ activation) (31). In humans, it is unknown which stimulation frequency is involved in the specific physiological and biochemical processes (13).

To date, narrative reviews have been published about the effects of NMES on gains in muscle performance, activation of motor units and/or muscle energetics $(14,26,32,33)$. However, there is a broad diversity in NMES programs, populations and outcomes which makes it difficult to interpret the conclusions. The effects of NMES on intramuscular changes have not been systematically reviewed yet. The purpose of this study is to systematically review changes in enzyme activity, muscle fiber type composition and muscle fiber size in human lower-limbs following a NMES program. A distinction will be made between HF-NMES and LF-NMES, as well as in healthy volunteers, patients with chronic organ failure or orthopedic problems. Our hypothesis is that LF-NMES $(\leq 20 \mathrm{~Hz})$ will primarily induce endurance training-like adaptations such as increased oxidative enzyme capacity and fiber type I proportion, whereas HF-NMES ( $\geq 50 \mathrm{~Hz}$ ) will primarily induce adaptations comparable to resistance training such as an increased glycolytic capacity, fiber type II proportion and muscle fiber size. Safety and the methodological quality of the trials will also be assessed.

\section{METHODS}

\section{DATA SOURCES AND SEARCHES}

We followed the procedures described in the PRISMA statement for reporting systematic reviews (34). A broad computerized literature search was performed to identify relevant trials reported in the English language. We used the following databases: Medline/PubMed (from 1966), EMBASE (from 1974), Cochrane Central Register of Controlled Trials (from 1898), CINAHL (from 1982), and The Physical Therapy Evidence Database (PEDro) (from 1982). Trials were collected up to March 2012. Search terms were combinations of keywords related to neuromuscular electrical stimulation, lower-limb muscles, muscle mass and muscle metabolic profile. The exact search algorithm for Medline/PubMed can be found in Appendix 3.1. Similar search algorithms 
were used for the other databases. In addition, reference lists and citations of original articles were also scanned to identify additional articles that may contain information on the topic of interest.

\section{Data extraction}

A pre-designed data abstraction form was used to obtain data on trial design and relevant results. For each article, characteristics of the study subjects were noted: a) the condition of the study population (healthy or primary diagnosis), gender and age; $b$ ) study design and NMES features (i.e., pulse duration, pulse frequency, duty cycle and pulse amplitude of the used current, training intensity, session time and duration in weeks); c) outcome measures, such as muscle enzyme activity (i.e., changes in oxidative and glycolytic enzymes), changes in muscle fiber type, changes in muscle fiber size and d) safety.

\section{Article selection}

Articles were used for further analyses when they met the following eligibility criteria:

\section{Types of studies}

Randomized controlled trials (RCTs), controlled clinical trials (CCTs) and clinical trials were included. A priori, congress abstracts, reviews, editorials and case reports were considered ineligible.

\section{Study subjects}

Included were trials in which human lower-limb muscles were electrically stimulated. Reasons for exclusion were studies with subjects suffering from neurological disorders (e.g. hemiplegia or lesion of the spine) or smooth muscle problems (e.g. period of bladder dysfunction)

\section{Types of interventions}

Included were trials in which the muscles were stimulated transcutaneously at the muscle motor points with a stimulation frequency of $\geq 20$ Hertz (LF-NMES) or $\geq 50$ Hertz (HF-NMES), a minimum total session time of 120 minutes, a minimum of 3 sessions per week in a minimum of 4 weeks $(13,14,18)$. Trials were not excluded based on pulse duration, pulse amplitude or training intensity.

\section{Types of outcome measures}

In the reviewed publications the outcome measures were muscular activities of enzymes involved in oxidative or glycolytic energy metabolism, changes in fiber type composition and/or muscle fiber size following NMES. 


\section{AsSeSSMENT OF METHOdological QUALITY}

The methodological quality of the identified trials was scored using the PEDro scale and is based on the Delphi list and "expert consensus" (35). The PEDro scale consists of 11 criteria which receives either a "yes" or a "no". Criterion 1 ('Eligibility criteria') is not used in the calculation of the PEDro score. All "yes" scores were summed resulting in a maximum score of 10 points (35). A k coefficient was used to measure the level of interrater reliability, using a method for comparing the level of reliability with categorical data along with their respective $95 \%$ confidence intervals (36). Consensus was sought in case of disagreement. Trials with a PEDro score of $\geq 6$ points were classified as "high-quality trials", while trials with a PEDro score $\leq 5$ points were classified as "low-quality trials" (37).

\section{Data analysis}

The use of meta-analytic techniques for data-pooling was not possible, because of the heterogeneity in study types, study populations, wide diversity in NMES protocols (e.g. frequency, pulse duration, session time, total number of sessions) and/or outcome parameters (e.g. activity of different enzymes). Also, the technique for measuring muscle fiber size differed among the included studies. Therefore, the present authors were only able to systematically review the available peer-reviewed literature and to critically appraise the methodological quality and the overall findings.

\section{RESULTS}

\section{SeArCh AND Selection}

After removing duplicates, 1,230 potentially relevant studies were identified by screening electronic databases. No trials were additionally identified by scanning reference lists. Of these trials, 1,171 were excluded based on title and/or abstract. Of the remaining 59 trials, 41 trials were excluded after reading the full text based on type of intervention, outcome parameters and/or publication type. Finally, 18 (38-55) trials were included. 8 trials $(39,40,46-48,52-54)$ studied changes in enzyme activity, 7 trials $(38,40,46-48,52,54)$ studied changes in muscle fiber type composition, and 14 trials $(38,40-45,48-52,54,55)$ studied changes in muscle fiber size following NMES (Figure 3.1).

A non-controlled experimental design was used in 6 studies, a controlled clinical trial was used in 3 studies and a randomized controlled design was used in 9 studies. 
Figure 3.1 Screening and selection process of trials.

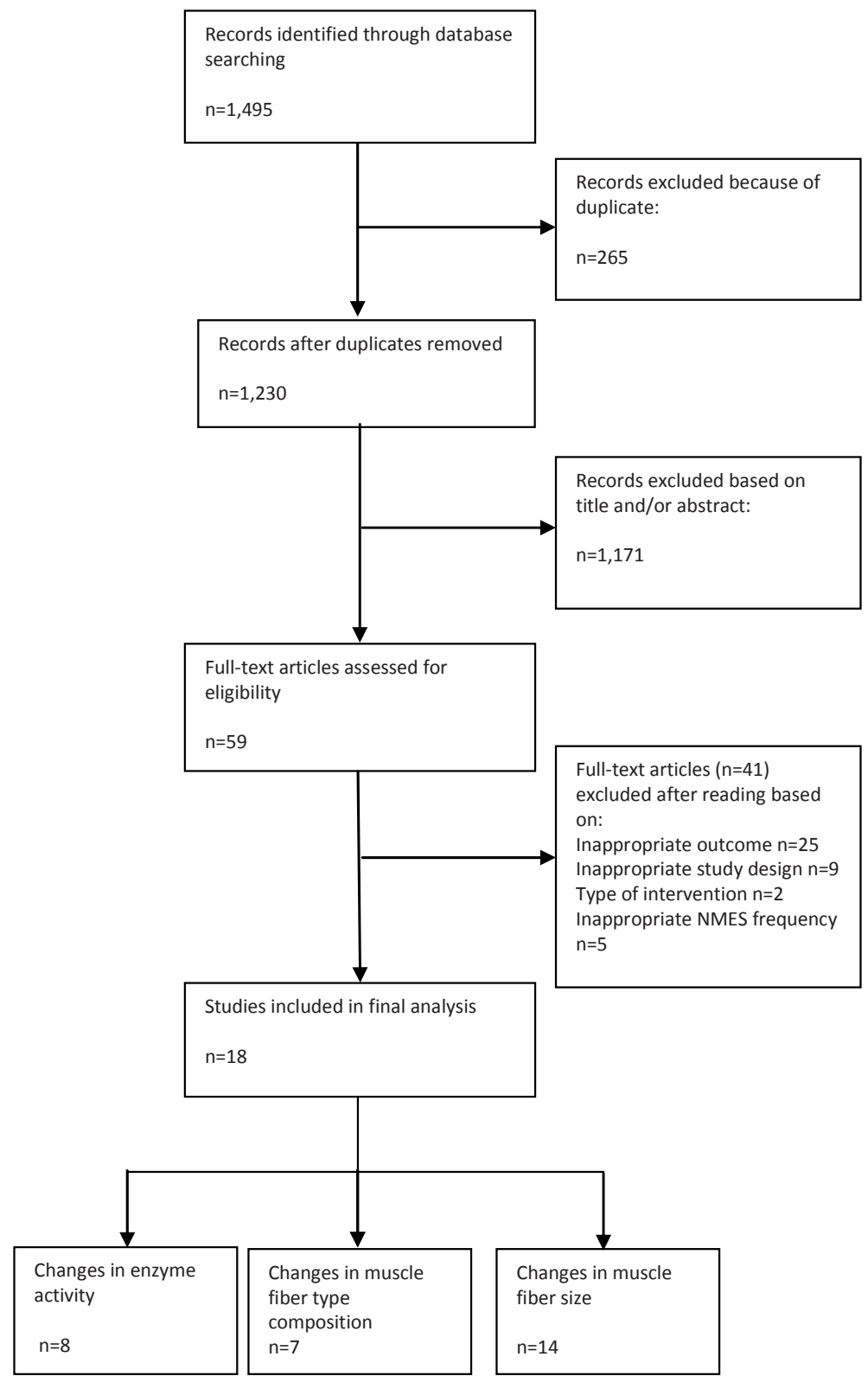




\section{Methodological quality of the tRials (TABle 3.1)}

The interrater reliability for the reviewers (MJHS and FMEF) was 0.74 ( $95 \%$ confidence interval, 0.68 to $0.80 ; p<0.001)$.

PEDro scores ranged from 2 to 9 points in the trials concerning changes in enzyme activity (median: 5.5 points). 4 trials $(50 \%)(46-48,54)$ scored $>6$ points on the PEDro scale. Of the trials studying muscle fiber type composition, PEDro scores ranged from 2 to 9 points (median: 6 points). 5 trials $(71 \%)(38,46-48,54)$ scored $>6$ points on the PEDro scale. In the trials concerning changes in muscle fiber size, PEDro scores ranged from 2 to 9 points (median: 4 points). 5 trials (36\%) $(38,48,49,54,55)$ scored $>6$ points on the PEDro scale.

Overall, eligibility criteria $(41,42,45,48,53)$, failure to conceal allocation, and blinding of the participants, therapists and outcome assessors were the most prevalent methodological shortcomings (Table 3.1).

\section{NMES PROTOCOLS (TABLE 3.2)}

There was a substantial heterogeneity in the studied populations and NMES features (Table 3.2). For example, 6 studies enrolled patients with chronic organ failure, divided in 2 studies $(38,54)$ including patients with chronic obstructive pulmonary disease (COPD) and 4 studies $(43,44,47,49)$ including patients with chronic heart failure (CHF). In 3 studies $(50,51,55)$ the subjects had orthopedic problems of the lower limbs and in 9 studies $(39-42,45,46,48,52,53)$ healthy volunteers were enrolled.

Different lower-limb muscles were stimulated in the identified trials: quadriceps femoris muscles $(38-42,48,50-53,55)$, calf muscles (45), quadriceps femoris muscles combined with calf muscles $(43,44,54)$, or quadriceps femoris muscles combined with hamstrings $(46,47,49)$.

All trials used biphasic impulse current forms ranging from 8 to 20 and 50 to 120 Hertz. Pulse duration, not reported in 1 study (53), ranged between 200 and $700 \mu$ s. Duty cycle, not reported in 1 study (53), ranged between 3 seconds on, 30 seconds off to 55 seconds on, 2 seconds off. Pulse amplitude, not reported in 1 study (39), varied between $10 \mathrm{~mA}$ until the individual's maximum level of toleration. Session time varied between 10 minutes and 8 hours, 1 to 2 times a day. The total number of sessions varied between 12 and 140 (Table 3.2) between 4 and 10 weeks. The total session time ranged from 2 to 384 hours. 
Changes in lower-limb skeletal muscle following neuromuscular electrical stimulation

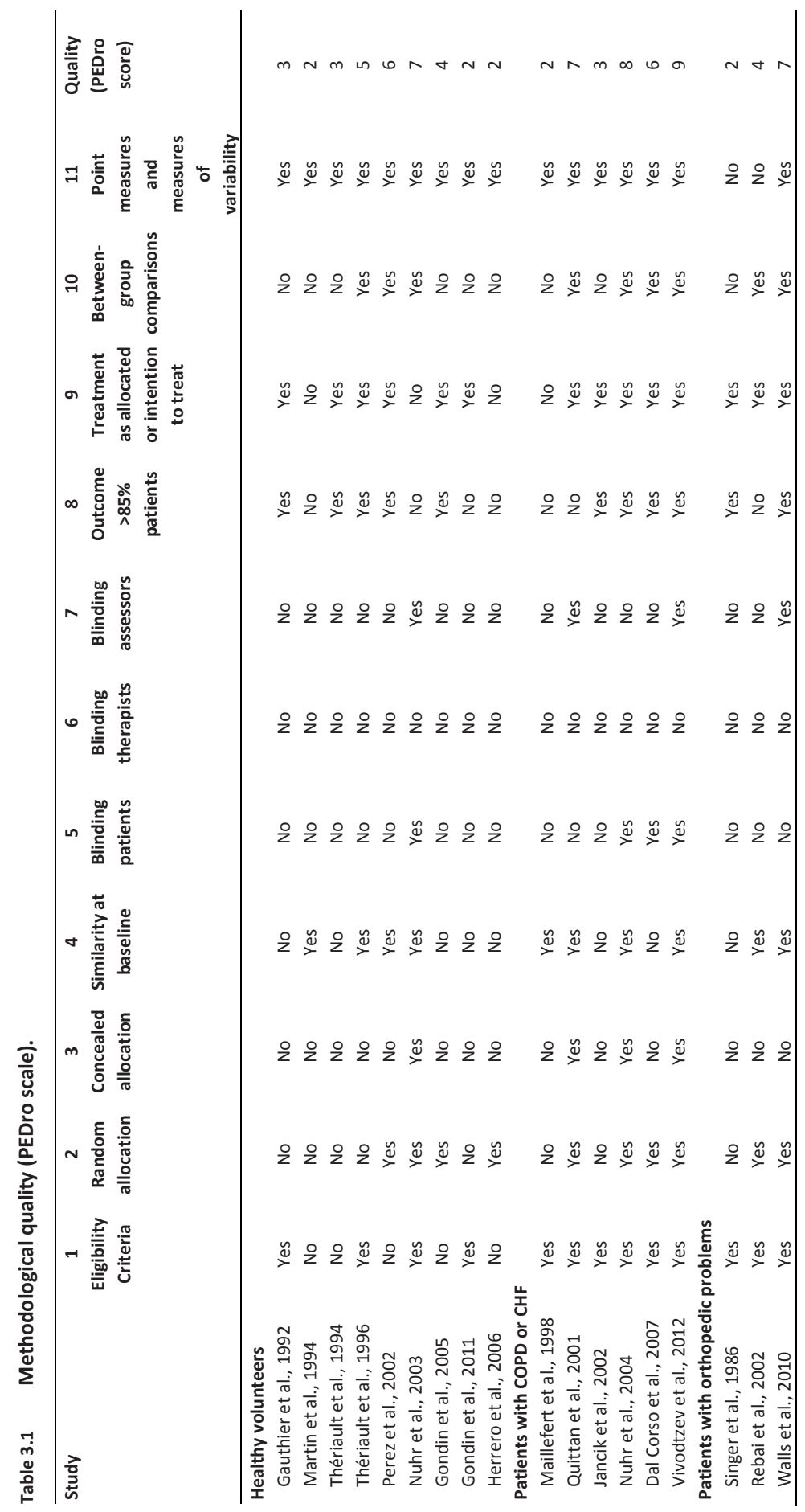




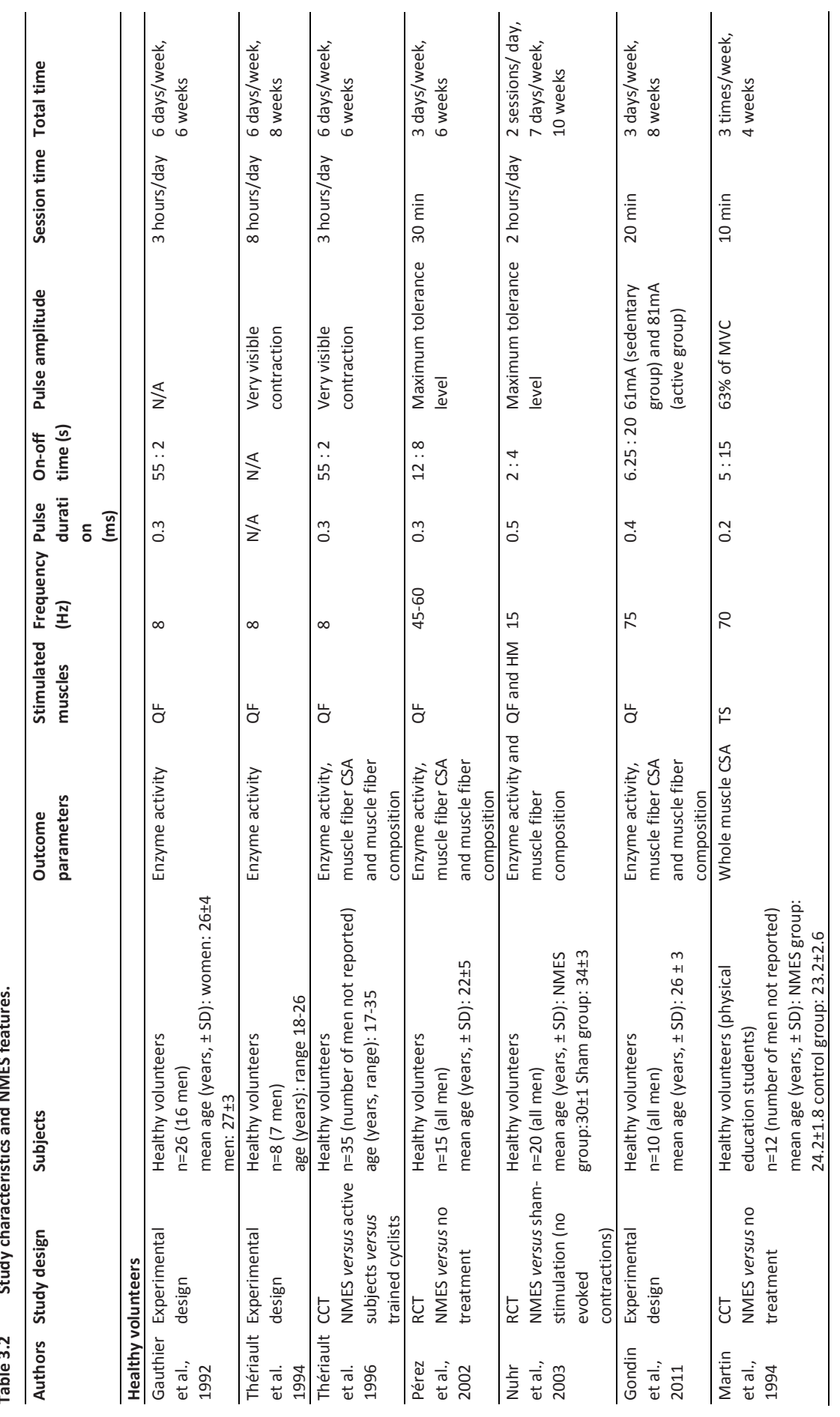




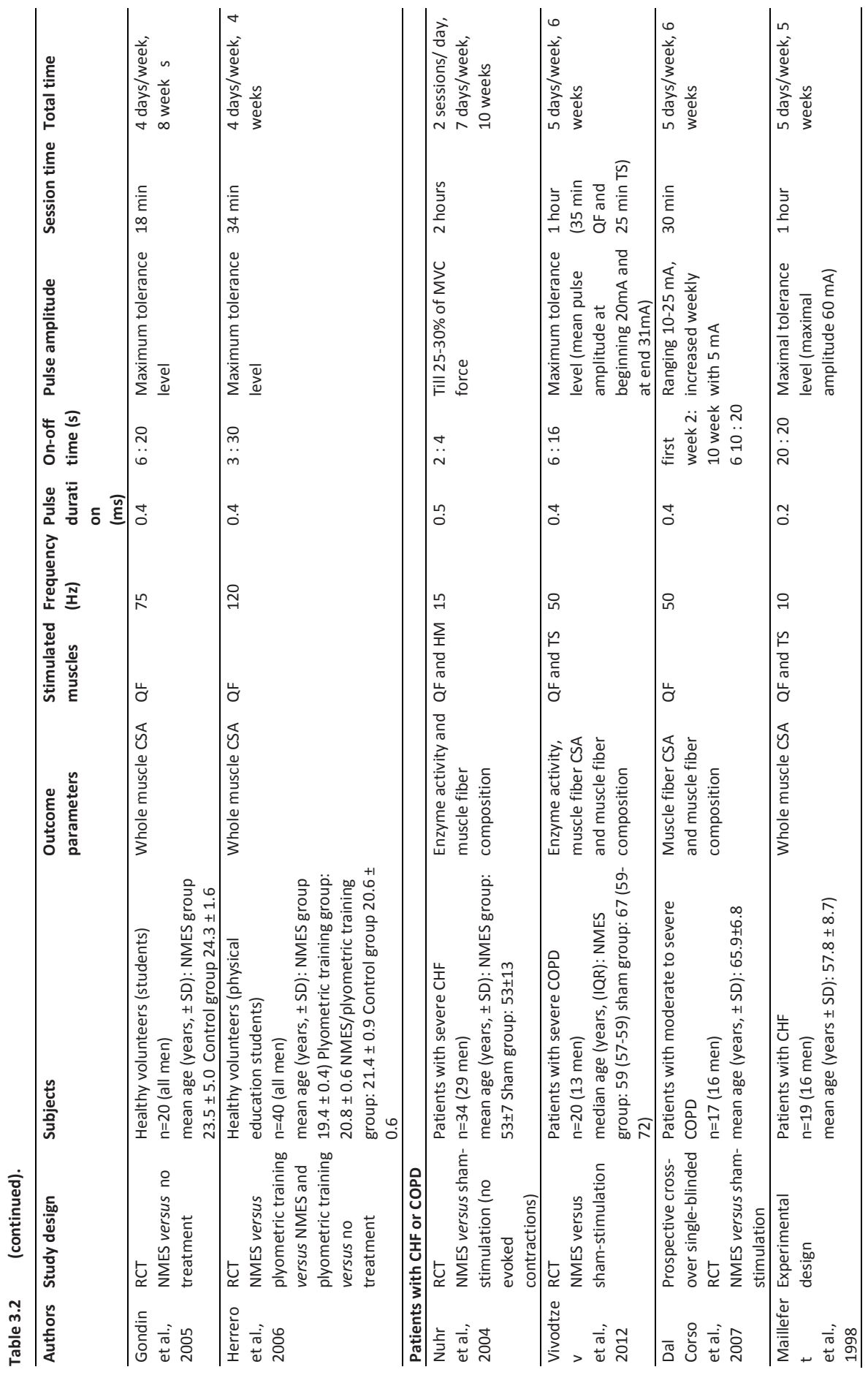




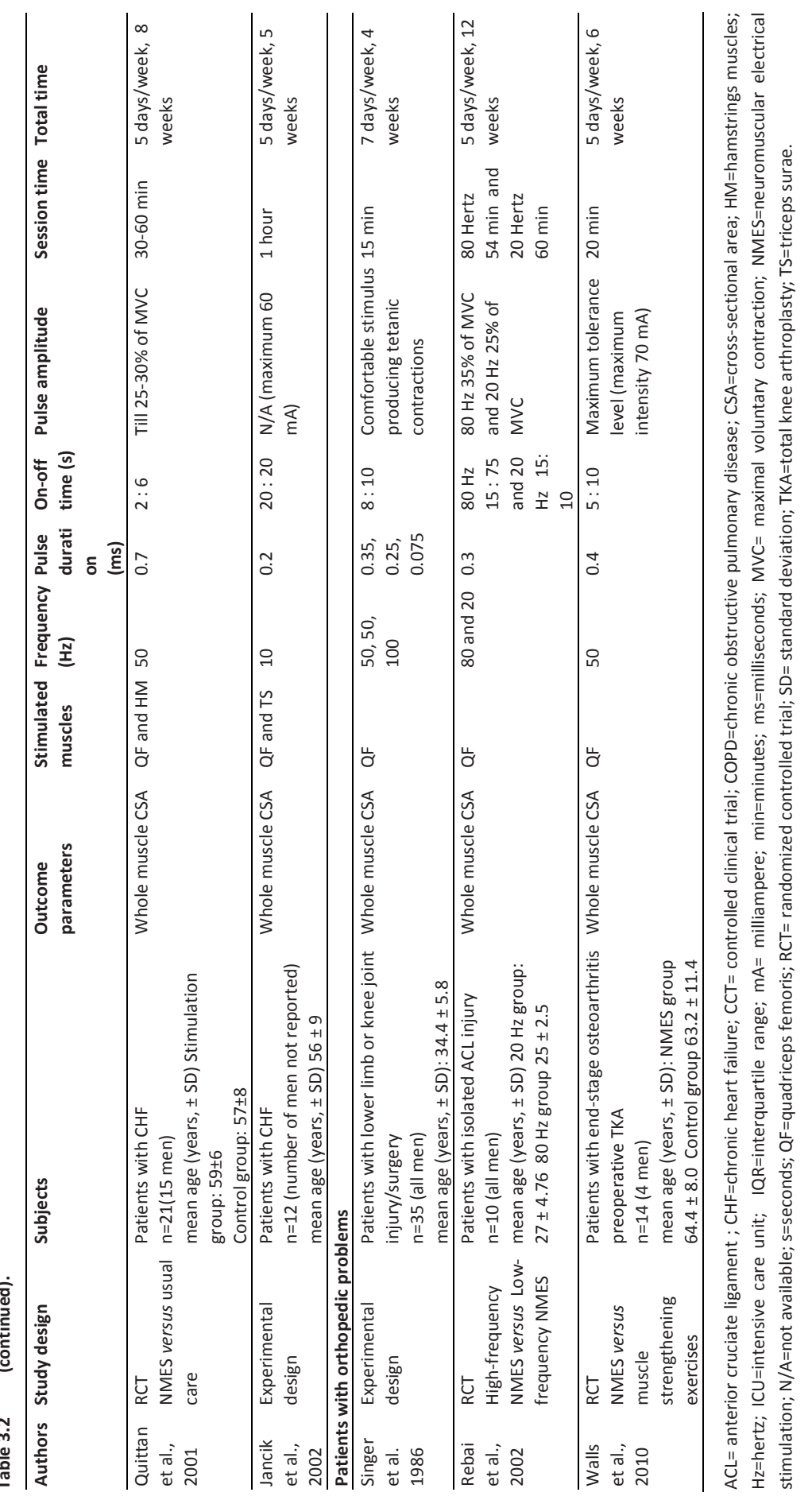




\section{SAFETY}

Safety was not reported in 13 trials. In 3 trials $(38,47,49)$ no relevant side effects or adverse events were reported. Only once (49) a delayed onset muscle soreness was reported and one trial explicitly reported the absence of serious discomfort in the stimulated subjects (41). Finally, in 1 trial (54) 1 study subject withdrew because of discomfort during NMES.

\section{Changes in enzyme activity following nMes}

Five trials studied changes in enzyme activity following LF-NMES $(39,46,47,52,53)$ and 3 trials following HF-NMES $(40,48,54)$ (Tables 3.3 and 3.4$)$. The study subjects consisted of healthy volunteers $(39,40,46,48,52,53)$, patients with severe CHF (47) or severe COPD (54). Enzyme activity was determined using muscle biopsies in the vastus lateralis of the quadriceps muscle in all studies.

\section{Changes in oxidative enzymes in healthy volunteers}

Levels of oxidative enzymes generally increased following LF-NMES (Table 3.3) and following HF-NMES (Table 3.4).

\section{Citrate synthase}

Citrate synthase (CS), a marker enzyme for the tricarboxylic acid cycle (Krebs cycle), was an outcome parameter in 4 LF-NMES trials $(39,46,52,53)$. In 3 trials $(39,52,53)$ CS increased compared to baseline ( 9 to $31 \%$ ) and in 1 trial (46) CS increased compared to sham-stimulation.

Isocitrate dehydrogenase

Isocitrate dehydrogenase, another enzyme that participates in the tricarboxylic acid cycle, increased significantly following HF-NMES compared to baseline (40).

\section{3-Hydroxylacyl-CoA dehydrogenase (HADH)}

$\mathrm{HADH}$, a key enzyme of ß-oxidation of fatty acids, increased significantly following LFNMES compared to baseline in 2 trials in healthy volunteers $(7-30 \%)(39,53)$. Contradictionary, in a HF-NMES trial (40) HADH decreased.

\section{Enoyl CoA hydratase}

Enoyl CoA hydratase, an enzyme that participates in the ß-oxidation of fatty acids, increased significantly following HF-NMES compared to baseline (40).

\section{NADH-ubiquinone oxidoreductase}

$\mathrm{NADH}$-ubiquinone oxidoreductase, complex I of the electron transport chain, increased significantly following HF-NMES compared to baseline (40). 


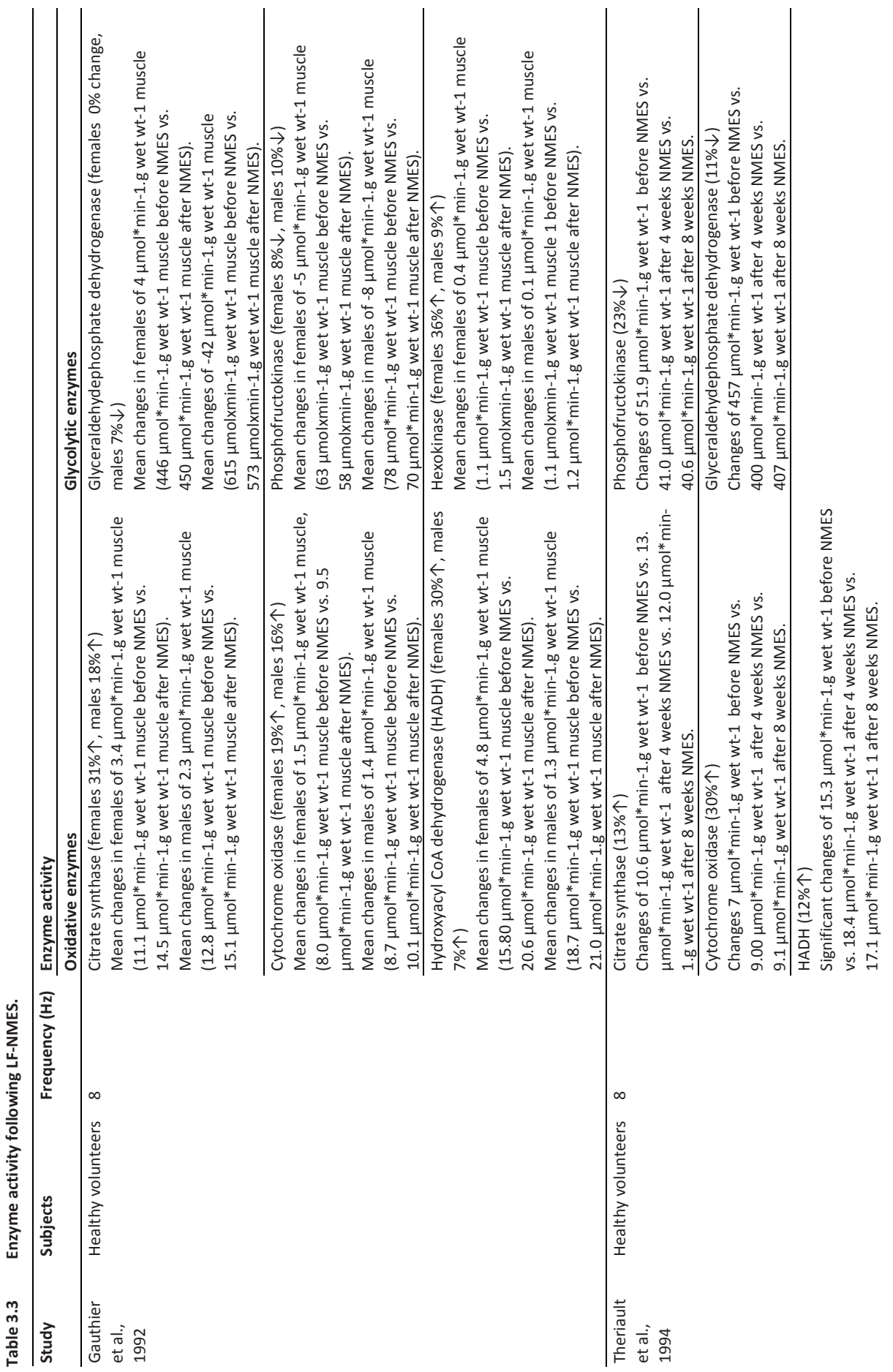




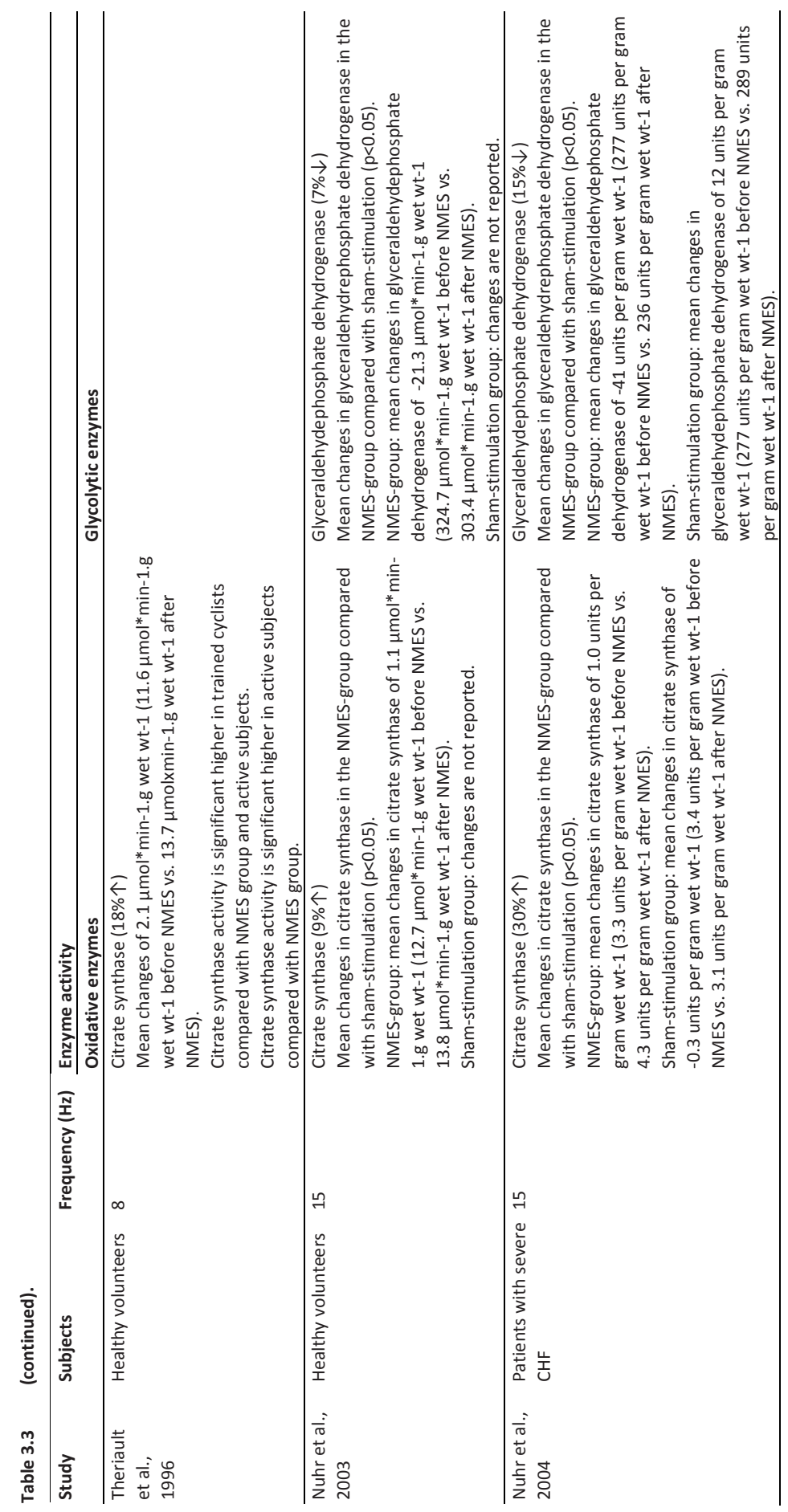




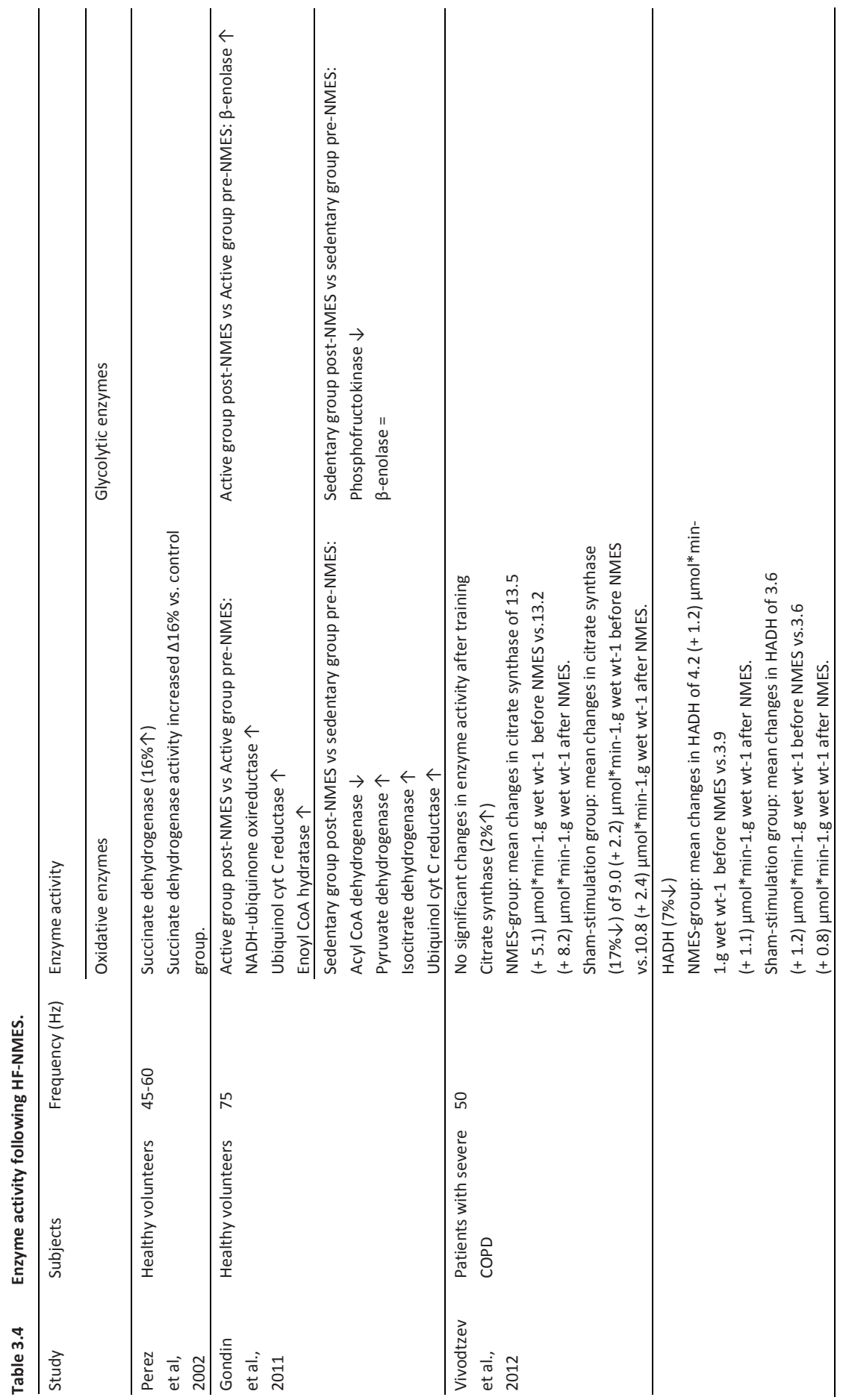




\section{Succinate dehydrogenase}

Succinate dehydrogenase, an enzyme that participates in both the tricarboxylic acid cycle and in complex II of the electron transport chain, increased significantly following HF-NMES compared to baseline and increased $16 \%$ compared to controls (48).

Ubiquinol-cytochrome c reductase

Ubiquinol cyt C reductase, complex III of the electron transport chain, increased significantly following HF-NMES compared to baseline (40).

\section{Cytochrome c oxidase}

Cytochrome c oxidase, complex IV of the electron-transfer chain metabolism, increased significantly following LF-NMES compared to baseline (16 to $19 \%)(39,53)$.

Pyruvate dehydrogenase.

Pyruvate dehydrogenase, an enzyme which contributes to linking the glycolysis metabolic pathway to the citric acid cycle and releasing energy via $\mathrm{NADH}$, increased significantly following HF-NMES compared to baseline (40).

\section{Changes in oxidative enzymes in patients with CHF or COPD}

Levels of CS increased following LF-NMES (15 Hertz) compared to sham-stimulation in patients with severe CHF (47) and did not change following HF-NMES (50 Hertz) in patients with severe COPD (54).

Levels of HADH did not change following HF-NMES (50 Hertz) in patients with severe COPD (54).

\section{Changes in glycolytic enzymes in healthy volunteers}

Levels of glycolytic enzymes generally did not change or decreased following LF-NMES or HF-NMES respectively (Tables 3.3 and 3.4).

\section{Phosphofructokinase (PFK)}

Levels of PFK, a glycolytic enzyme that catalyses the phosphorylation of fructose phosphate, decreased or did not change compared to baseline following LF-NMES or HF-NMES (variation from baseline was between -11 and $0 \%)(39,40,53)$.

Glyceraldehyde 3-phosphate dehydrogenase (GAPDH)

Concentrations of GAPDH, a marker enzyme of anaerobic energy metabolism by catalysing the sixth step of glycolysis, decreased significantly in 1 LF-NMES trial (46) compared with sham-stimulation (variation from baseline was $-15 \%$ ). Levels of GAPDH did not change in 2 LF-NMES trials $(39,53)$ compared to baseline. 


\section{Hexokinase}

Hexokinase, a key glycolytic enzyme, increased significantly in females (36\%) and did not change in males following LF-NMES compared to baseline (39).

\section{B-enolase}

B-enolase, which catalyses the glycolysis of 2-phosphoglycerate to phosphorenolpyruvate, did not change in a sedentary group of healthy young men following HF-NMES compared to baseline, but increased in an active group of healthy young men following HF-NMES compared to baseline (40).

\section{Changes in glycolytic enzymes in patients with CHF}

In patients with severe CHF levels of GAPDH decreased significantly in 1 LF-NMES trial (47) compared with sham-stimulation (variation from baseline was -15\%).

\section{SKELETAL MUSCLE FIBER TYPE COMPOSITION FOLLOWING NMES}

Three trials $(46,47,52)$ studied skeletal muscle fiber type composition following LFNMES and 4 trials $(38,40,48,54)$ following HF-NMES (Table 3.5$)$. The study subjects consisted of healthy volunteers $(40,46,48,52)$, patients with severe CHF (47) and patients with severe COPD $(38,54)$.

Table 3.5 Skeletal muscle fiber type composition following NMES.

\begin{tabular}{|c|c|c|c|c|c|c|}
\hline \multirow[t]{2}{*}{ Study } & \multirow[t]{2}{*}{ Subjects } & \multirow[t]{2}{*}{ Frequency } & \multicolumn{4}{|c|}{ Changes in muscle fiber type composition } \\
\hline & & & Type I fibers & Type II fibers & Type lla fibers & Type IIb/x fibers \\
\hline $\begin{array}{l}\text { Theriault } \\
\text { et al., } 1996\end{array}$ & $\begin{array}{l}\text { Healthy } \\
\text { volunteers }\end{array}$ & 8 & $=$ & & $19 \% \uparrow$ & $32 \% \downarrow$ \\
\hline $\begin{array}{l}\text { Nuhr } \\
\text { et al., } 2003\end{array}$ & $\begin{array}{l}\text { Healthy } \\
\text { volunteers }\end{array}$ & 15 & $15 \% \uparrow$ & & $=$ & $22 \% \downarrow$ \\
\hline $\begin{array}{l}\text { Perez } \\
\text { et al, } 2002\end{array}$ & $\begin{array}{l}\text { Healthy } \\
\text { volunteers }\end{array}$ & $45-60$ & $15 \% \downarrow$ & & $63 \% \uparrow$ & $88 \% \downarrow$ \\
\hline $\begin{array}{l}\text { Gondin } \\
\text { et al., } 2011\end{array}$ & $\begin{array}{l}\text { Healthy } \\
\text { volunteers }\end{array}$ & 75 & $\begin{array}{c}\text { Active group } \\
20 \% \uparrow \\
\text { Sedentary group } \\
96 \% \uparrow\end{array}$ & & $\begin{array}{c}\text { Active group } \\
9 \% \downarrow \\
\text { Sedentary } \\
\text { group } 42 \% \uparrow\end{array}$ & $\begin{array}{c}\text { Sedentary group } \\
79 \% \downarrow\end{array}$ \\
\hline $\begin{array}{l}\text { Nuhr } \\
\text { et al., } 2004\end{array}$ & $\begin{array}{l}\text { Patients with } \\
\text { CHF }\end{array}$ & 15 & $19 \% \uparrow$ & & $=$ & $19 \% \downarrow$ \\
\hline $\begin{array}{l}\text { Dal Corso et } \\
\text { al., } 2007\end{array}$ & $\begin{array}{l}\text { Patients with } \\
\text { moderate to } \\
\text { severe COPD }\end{array}$ & 50 & $4 \% \downarrow$ & $=$ & & \\
\hline $\begin{array}{l}\text { Vivodtzev et } \\
\text { al., } 2012\end{array}$ & $\begin{array}{l}\text { Patients with } \\
\text { severe COPD }\end{array}$ & 50 & $21 \% \downarrow$ & & $=$ & $=$ \\
\hline
\end{tabular}

Data are shown as variation from baseline. 


\section{Healthy volunteers}

Type I fibers

Proportion of type I fibers increased in 1 LF-NMES trial (15\%) (46) and 1 HF-NMES trial (active group 20\% and sedentary group 96\%) (40), and did not change in 1 LF-NMES trial (52). This fiber type decreased in 1 trial following HF-NMES (-15\%) (48).

\section{Type II fibers}

Type Ila fibers proportions increased following LF-NMES (19\%) (52) and HF-NMES (63\%) (48). In another HF-NMES trial this fiber type increased in the sedentary group (42\%) and decreased in the active group (9\%) (40).

Type IIx fibers proportions decreased in 2 LF-NMES trials $(22 \%$ and $32 \%)(46,52)$ and 2 HF-NMES trials (79\% and $88 \%)(40,48)$.

\section{Patients with CHF or COPD}

Proportion of type I fibers increased (19\%) following LF-NMES (47) and decreased (4\% and $21 \%$ ) following HF-NMES $(38,54)$, type II proportions did not change following HFNMES (38).

Type Ila proportions did not change in patients with CHF following LF-NMES (47) and these fiber type proportions remained unchanged compared to controls in patients with COPD following HF-NMES (54). Type IIx fibers decreased following LF-NMES (19\%) (47) and did not change following HF-NMES compared to a control group (54).

\section{Changes in Muscle size following nMes}

Different techniques were used to determine changes in whole muscle cross-sectional area (CSA) or muscle fiber CSA following NMES (Tables 3.6, 3.7 and 3.8). Muscle fiber CSA was measured by percutaneous needle biopsy of the vastus lateralis muscle $(38,40,48,52,54)$. Whole muscle CSA was measured by computed tomography $(45,49,51)$, magnetic resonance imaging (MRI) $(43,44,50,55)$, ultrasonography $(41)$ or circumference and skinfold measurements (42). Maillefert and colleagues determined the total volume of the soleus muscles and gastrocnemius muscles by calculated muscle volume from serial CSAs measured by MRI (44).

Three trials used LF-NMES $(43,44,52)$ and 11 trials used HF-NMES $(38,40-42,45$, 48-51,54,55).

\section{Healthy people}

Muscle fiber CSA did not change following 1 LF-NMES trial (52) and 1 HF-NMES trial (48). Following another HF-NMES trial muscle fiber CSA increased, in both type I and type II fibers (40) (Table 3.6). Whole muscle CSA was studied in 3 HF-NMES trials $(41,42,45)$ and did not change in 1 trial $(45)$ and increased in 2 trials $(41,42)$. 


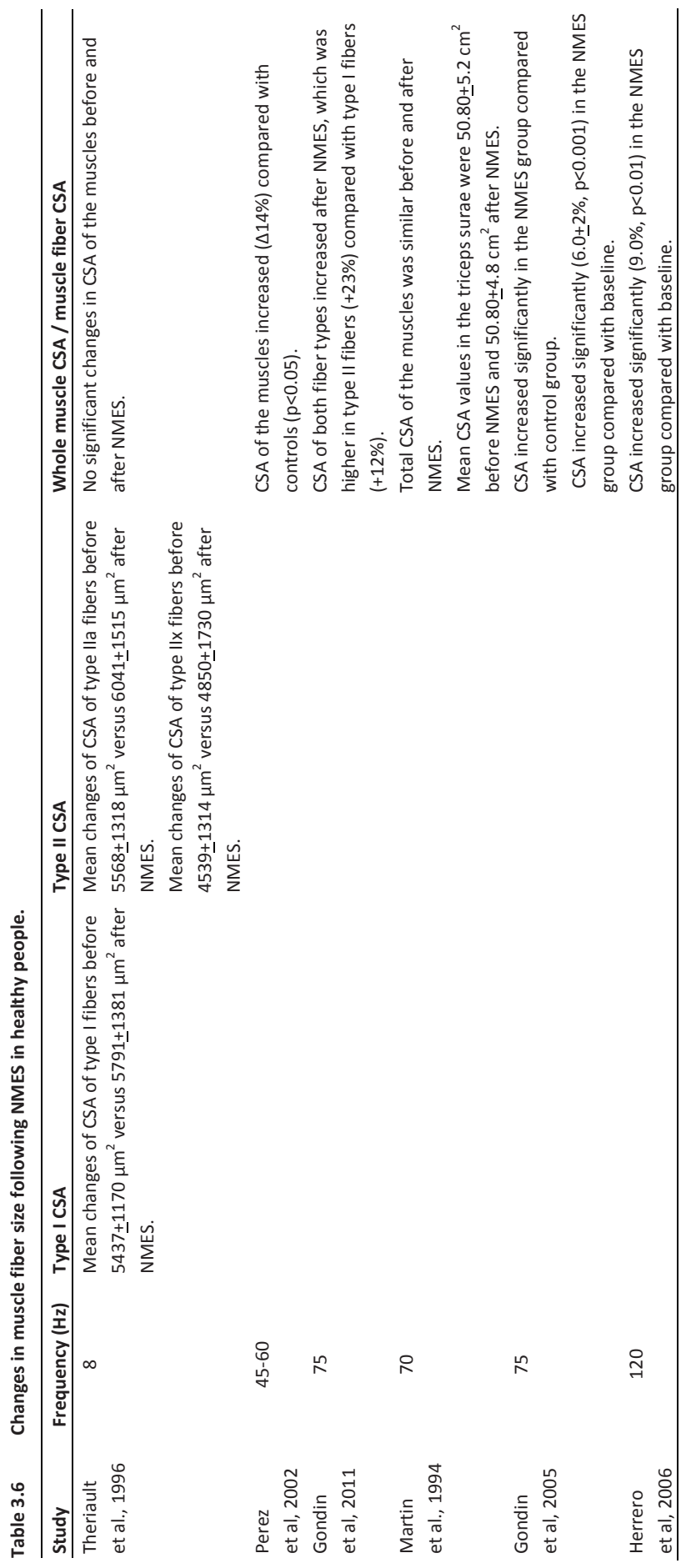




\section{Patients with CHF or COPD}

Following HF-NMES muscle fiber CSA did not change in one trial (38) and increased compared to sham stimulation in another trial (54) (Table 3.7). Whole muscle CSA increased following LF-NMES $(43,44)$ and HF-NMES (49) (Table 3.7).

\section{Patients with orthopedic problems}

Whole muscle CSA increased significantly in 1 HF-NMES trial (55) and did not change in two other HF-NMES trials $(50,51)$ (Table 3.8$)$.

\section{DISCUSSION}

This is the first systematic review on the effects of lower-limb NMES on intramuscular changes in the human lower-limb muscles. Most of the studies reported a significant increase in oxidative enzymes following LF-NMES. There are obvious changes in skeletal muscle fiber type composition following NMES. Indeed, LF-NMES seems to increase percentage of type I and Ila fibers, whereas fiber type composition following HF-NMES shows conflicting results. Both NMES protocols showed conflicting results in changes in muscle fiber size and total muscle volume. Heterogeneity in study design, study population, NMES features and outcome parameters prohibits the use of meta-analysis.

\section{Methodological considerations}

Overall, the methodological quality of the included trials was poor (median score 4 points). None of the 18 included trials had a perfect score on the PEDro scale (Table 3.2). In fact, only 7 trials (39\%) were of high-quality. Eligibility criteria were not specified in 4 trials (22\%) and a control group was lacking in 6 trials (33\%). Other methodological considerations were the limited number of study subjects ( $n=8$ to $\mathrm{n}=40$ ), the low mean age (38 years) and the fact that most subjects studied were men. Elderly subjects may respond differently on anabolic training stimulus compared to younger subjects (56). Moreover, gender-differences exist in fiber type distribution and mean CSA (57). So, the internal and external validity of the findings of the reviewed trials were limited.

\section{NMES PROTOCOLS}

NMES protocols varied tremendously among the included trials. Pulse duration, if reported, ranged between 200 and $700 \mu$ s. Pulse duration of 300-400 $\mu$ s is recommended for large muscle groups, such as the quadriceps muscles and calf muscles (58). It remains currently unknown which duty cycle is optimal for effective treatment. 
While throughout the literature a wide variety of protocols are used, there seems to be at least some agreement on the use of biphasic symmetrical pulses that last between 100 and $500 \mu$ s and are delivered at a pulse rate of $10-100 \mathrm{~Hz}$. Pulse rates between 10-50 $\mathrm{Hz}$ are used in patients with CHF and in patients with COPD with positive improvements in exercise capacity and health status $(23,59)$ whereas pulse rates between $50-100 \mathrm{~Hz}$ are mostly recommended for gains in muscle performance $(14,18)$. Such pulses are widely accepted as being well tolerated.

The stimulus intensity varied among the included trials, from a comfortable stimulus till maximum tolerance level. These diverse results could have been related to differences in protocols and stimulated muscles. It is strongly recommended that pulses are delivered at the highest tolerable pulse amplitude (22). Another common procedure is to quantify isometric maximal voluntary contraction (MVC) force at the beginning of a NMES session, and subsequently express the level of each electrically elicited contraction as a percentage of the MVC force (18).

The duration of the NMES programmes varied between 10 minutes to 8 hours/day, 1 to 2 sessions/day, 3 to 7 days/week for 4 to 10 weeks. The minimum total duration of the NMES in the included studies are in line with the studies which show significant improvements in peripheral muscle function $(13,14)$.

\section{Changes in Enzyme Activity following nMes}

Activity of oxidative enzymes generally increased significantly following 6 weeks of LF-NMES. The increase in oxidative enzyme activity was accompanied with an improved resistance to fatigue $(52,53)$ and an improvement in functional exercise capacity $(47)$. Compared with a minimum of 6 weeks of endurance cycling training $(60,61)$, the absolute and relative improvement in CS activity after NMES is lower.

Levels of glycolytic enzymes did not change or decreased following LF-NMES. These results are comparable with endurance training in healthy young men (62) and in patients with COPD (63). In healthy volunteers levels of oxidative enzymes increased following HF-NMES $(40,48)$. These adaptations are more endurance-specific. Collins and colleagues recently showed that the use of a wide pulse $(1 \mathrm{~ms})$, high frequency (80-100 Hz) and a low intensity might favour the recruitment of fatigue-resistant motor units (according to the Henneman's size principle) (64). This combination of stimulation parameters could also be relevant for increasing oxidative capacity. However, they used a wide pulse and low stimulation intensity whereas the included trials in the present review $(40,48)$ used narrow pulses and intensities at the maximum toleration level. As the consequence, the corresponding increase of oxidative enzymes is likely due to the non-selective recruitment of both type IIx and type I fibers during HF-NMES (17). Another study limitation is that only one study (in healthy volunteers) with a very small sample size (40) studied glycolytic enzymes following HF-NMES. In the sedentary group the downregulation of glycolytic enzymes is highly consistent with the fast-to-slow MHC isoform shift as slow fibers mostly have an oxidative metabolism and type Ilx fibers 
mostly have a glycolytic metabolism. Additionally, glycolytic enzyme content is known to increase in the order of slow oxidative, fast oxidative, glycolytic, fast glycolytic fibers (65). However, the small sample size is too limited $(n=10)$ to provide an answer on the hypothesis that HF-NMES increases glycolytic capacity.

\section{Changes in Skeletal muscle fiber type composition following nMes}

Changes in type I and Ila fiber proportion were variable following HF-NMES. Resistance training resulted in no changes in type I fibers and an increase of type II fibers $(7,66)$. However, based on the results of the present systematic review changes in type lla and type Ilx fibers following regular resistance training programs (67) cannot be compared with HF-NMES. Following LF-NMES type I and Ila fibers increased. Endurance training programs also resulted in an increase of type I and type lla fibers and a decrease of type Ilx fibers $(4,5,68)$. However, considering the small number of studies and heterogeneity in NMES protocols and study populations it is difficult to draw relations between LF-NMES and endurance training.

\section{Changes in MUSCle size following nMes}

In $55 \%$ of the HF-NMES studies muscle fiber size increased significantly. The increased CSA was accompanied with an increased muscle strength $(40-42,49)$. Changes in total muscle fiber size following LF-NMES are conflicting. Differences in the LF-NMES studies which could possible explain the conflicting results are the study population (e.g. age, healthy volunteers versus patients with chronic organ failure), measurement of muscle fiber size or intensity of NMES.

These results are in line with previous studies concluding that muscle fiber size increased less in subjects who performed endurance training than in strength training $(69,70)$. Conflicting results for the changes in muscle fiber size in HF-NMES could be related to the intensity of the training. In two trials $(38,51)$ reporting no changes in muscle fiber size, the stimulus intensity varied from "a comfortable stimulus" (51) to $25 \mathrm{~mA}$ (38) compared with a stimulus at the maximum tolerable level in the trials with an increased muscle fiber size (40-42,48). Moreover, Vivodtzev and colleagues showed that gains in muscle strength were proportional to the increase in pulse amplitude during the training program and to the final pulse amplitude of training (54). The impact of NMES is also dependent on the training duration. It is well known that long training duration is needed to induce muscle hypertrophy (71), however the total duration time in the HF-NMES trials ranged from 2 (45) to 54 hours (50).

\section{RECOMMENDATIONS}

Overall, LF-NMES seems to improve oxidative phenotype (oxidative enzyme capacities, type I/Ila fibers). However, some of the results of the included trials are difficult to 
compare and cannot be generalized. Besides the heterogeneity in NMES protocols and study designs, the number of study populations is limited and varies among the trials, from well-trained healthy volunteers $(40)$ to patients with severe chronic organ failure $(38,54)$ or severe orthopedic problems $(50,55)$. Stimulation variables (i.e., pulse amplitude, session time and number of sessions) might have influenced the number of muscle fibers recruited during NMES, the motor unit recruitment order and the types of the recruited muscle fibers $(18,72)$.

Therefore, future trials are needed to determine the optimal settings of NMES, such as stimulation frequency (HF-NMES or LF-NMES), session time, pulse amplitude and electrodes (number, size and location) in healthy (i.e. athletes) and in diseased people (i.e. COPD, CHF, orthopedic problems). These trials should not only study the effects of NMES versus volitional training, but also study the superimposed effects of NMES on volitional training.

Based on the results of the present systematic review, randomized controlled trials using concealed allocation, blinded therapists, blinded participants and blinded outcome assessors are recommended. Additionally, studies should focus on larger study populations, including both genders and a broad range in age. These studies should not include only healthy people but also people who are unable to perform or complete volitional exercise training programs. Finally, safety should be added to new randomized controlled trials as secondary outcome.

\section{CONCLUSION}

NMES seems to be a training modality resulting in changes in oxidative enzyme activity, skeletal muscle fiber type and skeletal muscle fiber size. A more formal meta-analysis would be a more rigorous way to analyze the current data, but is not possible at this time. Indeed, the small sample sizes, the variance in study populations, the nonrandomized controlled study designs, the variance in primary outcomes and the large heterogeneity in NMES protocols are major methodological limitations which may limit the external validity of the current findings. Therefore, it is difficult to draw definitive conclusions about the effects of stimulation frequencies on muscular changes. This systematic review, however, will help generate discussion in the field that would lead to a consensus in study design that would permit a meta-analysis in the future.

A better understanding of metabolic and structural changes following NMES is of particular clinical interest as it will increase its applicability in specific populations who are not able to perform regular exercise training. Therefore, future well-designed, randomized controlled trials with larger study samples are needed to determine the optimal NMES settings (i.e. electrode placement, stimulation frequency and pulse amplitude) to achieve endurance or resistance training-like adaptations. The actual stimulation parameters, session time, total time and changes in NMES pulse intensity over time should be reported to enable comparisons between studies and to facilitate the further development and implementation of NMES. 


\section{REFERENCES}

1. American College of Sports Medicine, Position Stand. The recommended quantity and quality of exercise for developing and maintaining cardiorespiratory and muscular fitness, and flexibility in healthy adults. Med Sci Sports Exerc. 1998;30(6):975-91.

2. Bell GJ, Syrotuik D, Martin TP, Burnham R, Quinney HA. Effect of concurrent strength and endurance training on skeletal muscle properties and hormone concentrations in humans. Eur J Appl Physiol. 2000;81(5):418-27.

3. Hakkinen K, Alen M, Kraemer WJ, Gorostiaga E, Izquierdo M, Rusko H, et al. Neuromuscular adaptations during concurrent strength and endurance training versus strength training. Eur J Appl Physiol. 2003;89(1):42-52.

4. Carter SL, Rennie CD, Hamilton SJ, Tarnopolsky. Changes in skeletal muscle in males and females following endurance training. Can J Physiol Pharmacol. 2001;79(5):386-92.

5. Trappe SW, Costill DL, Fink WJ, Pearson DR. Skeletal muscle characteristics among distance runners: a 20-yr follow-up study. J Appl Physiol. 1995;78(3):823-9.

6. Tesch PA, Thorsson A, Essen-Gustavsson B. Enzyme activities of FT and ST muscle fibers in heavyresistance trained athletes. J Appl Physiol. 1989;67(1):83-7.

7. Kryger Al, Andersen JL. Resistance training in the oldest old: consequences for muscle strength, fiber types, fiber size, and MHC isoforms. Scand J Med Sci Sports. 2007;17(4):422-30.

8. Andersen JL, Aagaard P. Myosin heavy chain IIX overshoot in human skeletal muscle. Muscle Nerve. 2000;23(7):1095-104.

9. Farup J, Kjolhede T, Sorensen H, Dalgas U, Moller AB, Vestergaard PF, et al. Muscle morphological and strength adaptations to endurance vs. resistance training. J Strength Cond Res. 2012;26(2):398-407.

10. Verdijk LB, Gleeson BG, Jonkers RA, Meijer K, Savelberg HH, Dendale P, et al. Skeletal muscle hypertrophy following resistance training is accompanied by a fiber type-specific increase in satellite cell content in elderly men. J Gerontol A Biol Sci Med Sci. 2009;64(3):332-9.

11. Hainaut K, Duchateau J. Neuromuscular electrical stimulation and voluntary exercise. Sports Med. 1992;14(2):100-13.

12. Vanderthommen M, Crielaard JM. [Muscle electric stimulation in sports medicine]. Rev Med Liege. 2001;56(5):391-5. Electromyostimulation en medecine du sport.

13. Vivodtzev I, Lacasse $Y$, Maltais F. Neuromuscular electrical stimulation of the lower limbs in patients with chronic obstructive pulmonary disease. J Cardiopulm Rehabil Prev. 2008;28(2):79-91.

14. Vanderthommen $M$, Duchateau J. Electrical stimulation as a modality to improve performance of the neuromuscular system. Exerc Sport Sci Rev. 2007;35(4):180-5.

15. Vanderthommen M, Duteil S, Wary C, Raynaud JS, Leroy-Willig A, Crielaard JM, et al. A comparison of voluntary and electrically induced contractions by interleaved $1 \mathrm{H}$ - and 31P-NMRS in humans. J Appl Physiol. 2003;94(3):1012-24.

16. Bickel CS, Slade J, Mahoney E, Haddad F, Dudley GA, Adams GR. Time course of molecular responses of human skeletal muscle to acute bouts of resistance exercise. J Appl Physiol. 2005;98(2):482-8.

17. Bickel CS, Gregory CM, Dean JC. Motor unit recruitment during neuromuscular electrical stimulation: a critical appraisal. Eur J Appl Physiol. 2011;111(10):2399-407.

18. Maffiuletti NA. Physiological and methodological considerations for the use of neuromuscular electrical stimulation. Eur J Appl Physiol. 2010;110(2):223-34.

19. Cabric M, Appell HJ, Resic A. Fine structural changes in electrostimulated human skeletal muscle. Evidence for predominant effects on fast muscle fibres. Eur J Appl Physiol Occup Physiol. 1988;57(1): 1-5.

20. Trimble $\mathrm{MH}$, Enoka RM. Mechanisms underlying the training effects associated with neuromuscular electrical stimulation. Phys Ther. 1991;71(4):273-80; discussion 80-2.

21. Gerovasili V, Stefanidis K, Vitzilaios K, Karatzanos E, Politis P, Koroneos A, et al. Electrical muscle stimulation preserves the muscle mass of critically ill patients: a randomized study. Crit Care. 2009;13(5):R161.

22. Lake DA. Neuromuscular electrical stimulation. An overview and its application in the treatment of sports injuries. Sports Med. 1992;13(5):320-36. 
23. Sillen MJ, Speksnijder CM, Eterman RM, Janssen PP, Wagers SS, Wouters EF, et al. Effects of neuromuscular electrical stimulation of muscles of ambulation in patients with chronic heart failure or COPD: a systematic review of the English-language literature. Chest. 2009;136(1):44-61.

24. Sillen MJ, Janssen PP, Akkermans MA, Wouters EF, Spruit MA. The metabolic response during resistance training and neuromuscular electrical stimulation (NMES) in patients with COPD, a pilot study. Respir Med. 2008;102(5):786-9.

25. Chan Kwan Kit-Lan P. Contemporary trends in electrical stimulation: "The frequency-specificity theory". Hong-Kong Physiother J. 1991/1992;13:23-7.

26. Maffiuletti NA. Physiological and methodological considerations for the use of neuromuscular electrical stimulation. Eur J Appl Physiol. 2010.

27. Grimby L, Hannerz J. Firing rate and recruitment order of toe extensor motor units in different modes of voluntary conraction. J Physiol. 1977;264(3):865-79.

28. Taylor A, Stephens JA. Study of human motor unit contractions by controlled intramuscular microstimulation. Brain research. 1976;117(2):331-5.

29. Edwards RH, Young A, Hosking GP, Jones DA. Human skeletal muscle function: description of tests and normal values. Clinical science and molecular medicine. 1977;52(3):283-90.

30. Kramer JF. Effect of electrical stimulation current frequencies on isometric knee extension torque. Phys Ther. 1987;67(1):31-8.

31. Atherton PJ, Babraj J, Smith K, Singh J, Rennie MJ, Wackerhage H. Selective activation of AMPK-PGC1alpha or PKB-TSC2-mTOR signaling can explain specific adaptive responses to endurance or resistance training-like electrical muscle stimulation. FASEB journal : official publication of the Federation of American Societies for Experimental Biology. 2005;19(7):786-8.

32. Gregory $\mathrm{CM}$, Bickel CS. Recruitment patterns in human skeletal muscle during electrical stimulation. Phys Ther. 2005;85(4):358-64.

33. Gondin J, Cozzone PJ, Bendahan D. Is high-frequency neuromuscular electrical stimulation a suitable tool for muscle performance improvement in both healthy humans and athletes? Eur J Appl Physiol. 2011;111(10):2473-87.

34. Liberati A, Altman DG, Tetzlaff J, Mulrow C, Gotzsche PC, loannidis JP, et al. The PRISMA statement for reporting systematic reviews and meta-analyses of studies that evaluate healthcare interventions: explanation and elaboration. BMJ. 2009;339:b2700.

35. Sherrington C, Herbert RD, Maher CG, Moseley AM. PEDro. A database of randomized trials and systematic reviews in physiotherapy. Man Ther. 2000;5(4):223-6.

36. Brennan $P$, Silman A. Statistical methods for assessing observer variability in clinical measures. BMJ. 1992;304(6840):1491-4.

37. Foley NC, Bhogal SK, Teasell RW, Bureau Y, Speechley MR. Estimates of quality and reliability with the physiotherapy evidence-based database scale to assess the methodology of randomized controlled trials of pharmacological and nonpharmacological interventions. Phys Ther. 2006;86(6):817-24.

38. Dal Corso S, Napolis L, Malaguti C, Gimenes AC, Albuquerque A, Nogueira CR, et al. Skeletal muscle structure and function in response to electrical stimulation in moderately impaired COPD patients. Respir Med. 2007;101(6):1236-43.

39. Gauthier JM, Theriault R, Theriault G, Gelinas Y, Simoneau JA. Electrical stimulation-induced changes in skeletal muscle enzymes of men and women. Med Sci Sports Exerc. 1992;24(11):1252-6.

40. Gondin J, Brocca L, Bellinzona E, D'Antona G, Maffiuletti NA, Miotti D, et al. Neuromuscular electrical stimulation training induces atypical adaptations of the human skeletal muscle phenotype: a functional and proteomic analysis. J Appl Physiol. 2011;110(2):433-50.

41. Gondin J, Guette M, Ballay Y, Martin A. Electromyostimulation training effects on neural drive and muscle architecture. Med Sci Sports Exerc. 2005;37(8):1291-9.

42. Herrero JA, Izquierdo M, Maffiuletti NA, Garcia-Lopez J. Electromyostimulation and plyometric training effects on jumping and sprint time. Int J Sports Med. 2006;27(7):533-9.

43. Jancik J, Siegelova J, Homolka P, Svacinova H, Placheta P, Varnayova L, et al. Low-frequency electrical stimulation of skeletal muscles in patients with chronic heart failure. . Scripta Medica Facultatis Medicae Universitatis Brunensis Masarykianae. 2002;75(4):203-8. 
44. Maillefert JF, Eicher JC, Walker P, Dulieu V, Rouhier-Marcer I, Branly F, et al. Effects of low-frequency electrical stimulation of quadriceps and calf muscles in patients with chronic heart failure. J Cardiopulm Rehabil. 1998;18(4):277-82.

45. Martin L, Cometti G, Pousson M, Morlon B. The influence of electrostimulation on mechanical and morphological characteristics of the triceps surae. J Sports Sci. 1994;12(4):377-81.

46. Nuhr M, Crevenna R, Gohlsch B, Bittner C, Pleiner J, Wiesinger G, et al. Functional and biochemical properties of chronically stimulated human skeletal muscle. Eur J Appl Physiol. 2003;89(2):202-8.

47. Nuhr MJ, Pette D, Berger R, Quittan M, Crevenna R, Huelsman M, et al. Beneficial effects of chronic low-frequency stimulation of thigh muscles in patients with advanced chronic heart failure. Eur Heart J. 2004;25(2):136-43.

48. Perez M, Lucia A, Rivero JL, Serrano AL, Calbet JA, Delgado MA, et al. Effects of transcutaneous shortterm electrical stimulation on $\mathrm{M}$. vastus lateralis characteristics of healthy young men. Pflugers Arch. 2002;443(5-6):866-74.

49. Quittan M, Wiesinger GF, Sturm B, Puig S, Mayr W, Sochor A, et al. Improvement of thigh muscles by neuromuscular electrical stimulation in patients with refractory heart failure: a single-blind, randomized, controlled trial. Am J Phys Med Rehabil. 2001;80(3):206-14; quiz 15-6, 24.

50. Rebai H, Barra V, Laborde A, Bonny JM, Poumarat G, Coudert J. Effects of two electrical stimulation frequencies in thigh muscle after knee surgery. Int J Sports Med. 2002;23(8):604-9.

51. Singer K. The influence of unilataral electrical stimulation on motor unit activity patterns in atrophic human quadriceps. The Australian Journal of Physiotherapy. 1986;32:31-7.

52. Theriault R, Boulay MR, Theriault G, Simoneau JA. Electrical stimulation-induced changes in performance and fiber type proportion of human knee extensor muscles. Eur J Appl Physiol Occup Physiol. 1996;74(4):311-7.

53. Theriault R, Theriault G, Simoneau JA. Human skeletal muscle adaptation in response to chronic lowfrequency electrical stimulation. J Appl Physiol. 1994;77(4):1885-9.

54. Vivodtzev I, Debigare R, Gagnon P, Mainguy V, Saey D, Dube A, et al. Functional and Muscular Effects of Neuromuscular Electrical Stimulation in Patients With Severe COPD: A Randomized Clinical Trial. Chest. 2012;141(3):716-25.

55. Walls RJ, McHugh G, O'Gorman DJ, Moyna NM, O'Byrne JM. Effects of preoperative neuromuscular electrical stimulation on quadriceps strength and functional recovery in total knee arthroplasty. A pilot study. BMC Musculoskelet Disord. 2010;11:119.

56. Canepari M, Rossi R, Pellegrino MA, Orrell RW, Cobbold M, Harridge S, et al. Effects of resistance training on myosin function studied by the in vitro motility assay in young and older men. J Appl Physiol. 2005;98(6):2390-5.

57. Simoneau JA, Bouchard C. Human variation in skeletal muscle fiber-type proportion and enzyme activities. Am J Physiol. 1989;257(4 Pt 1):E567-72.

58. Bowman BR, Baker LL. Effects of waveform parameters on comfort during transcutaneous neuromuscular electrical stimulation. Ann Biomed Eng. 1985;13(1):59-74.

59. Smart NA, Dieberg G, Giallauria F. Functional electrical stimulation for chronic heart failure: A metaanalysis. Int J Cardiol. 2012.

60. Fernstrom M, Tonkonogi M, Sahlin K. Effects of acute and chronic endurance exercise on mitochondrial uncoupling in human skeletal muscle. J Physiol. 2004;554(Pt 3):755-63.

61. Howarth KR, LeBlanc PJ, Heigenhauser GJ, Gibala MJ. Effect of endurance training on muscle TCA cycle metabolism during exercise in humans. J Appl Physiol. 2004;97(2):579-84.

62. Tremblay A, Simoneau JA, Bouchard C. Impact of exercise intensity on body fatness and skeletal muscle metabolism. Metabolism: clinical and experimental. 1994;43(7):814-8.

63. Maltais F, LeBlanc P, Simard C, Jobin J, Berube C, Bruneau J, et al. Skeletal muscle adaptation to endurance training in patients with chronic obstructive pulmonary disease. Am J Respir Crit Care Med. 1996;154(2 Pt 1):442-7.

64. Collins DF, Burke D, Gandevia SC. Sustained contractions produced by plateau-like behaviour in human motoneurones. J Physiol. 2002;538(Pt 1):289-301.

65. Takekura H, Yoshioka T. Determination of metabolic profiles on single muscle fibres of different types. J Muscle Res Cell Motil. 1987;8(4):342-8. 
66. Hakkinen K, Newton RU, Gordon SE, McCormick M, Volek JS, Nindl BC, et al. Changes in muscle morphology, electromyographic activity, and force production characteristics during progressive strength training in young and older men. J Gerontol A Biol Sci Med Sci. 1998;53(6):B415-23.

67. Holm L, Reitelseder S, Pedersen TG, Doessing S, Petersen SG, Flyvbjerg A, et al. Changes in muscle size and $\mathrm{MHC}$ composition in response to resistance exercise with heavy and light loading intensity. J Appl Physiol. 2008;105(5):1454-61.

68. Short KR, Vittone JL, Bigelow ML, Proctor DN, Coenen-Schimke JM, Rys P, et al. Changes in myosin heavy chain mRNA and protein expression in human skeletal muscle with age and endurance exercise training. J Appl Physiol. 2005;99(1):95-102.

69. Hakkinen K, Keskinen KL. Muscle cross-sectional area and voluntary force production characteristics in elite strength- and endurance-trained athletes and sprinters. Eur J Appl Physiol Occup Physiol. 1989;59(3):215-20.

70. Putman CT, Xu X, Gillies E, MacLean IM, Bell GJ. Effects of strength, endurance and combined training on myosin heavy chain content and fibre-type distribution in humans. Eur J Appl Physiol. 2004;92(45):376-84.

71. American College of Sports Medicine position stand. Progression models in resistance training for healthy adults. Med Sci Sports Exerc. 2009;41(3):687-708.

72. Bergquist AJ, Clair JM, Lagerquist O, Mang CS, Okuma Y, Collins DF. Neuromuscular electrical stimulation: implications of the electrically evoked sensory volley. Eur J Appl Physiol. 2011;111(10):2409-26. 


\section{APPENDIX 3.1}

SEARCHSTRING Medline/PubMed 02-03-2012:

("neuromuscular electrical stimulation" OR "functional electrical stimulation" OR "muscle stimulation" OR "transcutaneous electrical stimulation" OR electrophysiology OR electrotherapy OR neurostimulation OR "electric stimulation" OR "electrical stimulation" OR elektromyostimulation OR electromyostimulation OR electrostimulation OR "muscle stimulation" OR NMES OR FES OR TENS OR EMS) AND (quadriceps OR vastus OR "knee extensor" OR "knee flexor" OR "triceps surae" OR gastrocnemius OR calf OR tibialis OR soleus OR hamstring) AND (muscle AND ("Muscle Fibers, Fast-Twitch" OR "Muscle Fibers, Slow-Twitch" OR biopsy OR biopsies OR "Enzymes" OR enzymes OR fibers OR fibres OR "muscle mass" OR volume OR "cross sectional area" OR CSA)) AND ("randomized controlled trial" OR "controlled clinical trial" OR OR "clinical trial" OR trial OR RCT OR CCT OR weeks OR wk OR week OR month OR training OR rehabilitation) NOT (review [pt] OR letter [pt] OR animal OR rat OR mouse OR mice OR equine OR horse) 



\section{CHAPTER 4}

The metabolic response during resistance training

and neuromuscular electrical stimulation (NMES) in patients with COPD, a pilot study

Maurice JH Sillen, Paul P Janssen, Marco A Akkermans, Emiel FM Wouters, Martijn A Spruit

Respiratory Medicine 2008;102:786-789 


\section{ABSTRACT}

Resistance training and transcutaneous neuromuscular electrical stimulation (NMES) are new modalities in rehabilitation of severely disabled patients with chronic obstructive pulmonary disease (COPD). The purpose of this study was to compare the metabolic response during resistance training and during NMES of the quadriceps femoris muscles in patients with COPD entering pulmonary rehabilitation.

Pulmonary function, body composition, peak aerobic capacity, the Medical Research Council dyspnea grade, and the one-repetition maximum strength assessment were evaluated in 13 COPD patients. Additionally, peak oxygen uptake, peak minute ventilation, and Borg symptom scores were assessed during a resistance training session and a NMES session.

The median peak oxygen uptake and median peak minute ventilation during the resistance training session were significantly higher compared to the NMES session. In addition, these higher metabolic responses were accompanied by higher symptom Borg scores for dyspnea and leg fatigue.

To conclude, the metabolic response was significantly lower during a NMES session compared to a resistance exercise training session in patients with COPD. Nevertheless, both modalities seem to result in an acceptable metabolic response accompanied by a clinically acceptable sensation of dyspnea and leg fatigue. 


\section{INTRODUCTION}

Patients with chronic obstructive pulmonary disease (COPD) experience exercise intolerance, dyspnea and a decreased health status, in spite of an optimal pharmacological treatment (1).

Endurance training alone has been shown to improve health status, exercise tolerance, and peripheral muscle function in $\operatorname{COPD}(2,3)$. Unfortunately, not every COPD patient is able to complete high-intensity endurance training, such as ergometry leg cycling and treadmill walking, due to exercise-induced dyspnea and/or muscle fatigue $(4,5)$. Actually, a decreased peripheral muscle force and depletion of muscle mass have been shown to be related to exercise intolerance in patients with COPD, irrespective of the degree of airflow limitation $(6,7)$. It therefore seems reasonable to add new treatment modalities to pulmonary rehabilitation to complement endurance training (8). Resistance training and transcutaneous neuromuscular electrical stimulation (NMES) are new modalities, which have been shown to have positive effects on skeletal muscle function, exercise tolerance, and disease-specific health status in patients with severe to very severe COPD, and are well tolerated without creating the overt sensation of dyspnea (9-12). This is most probably due to a relatively low load on the (severely) impaired respiratory system by exercising a smaller skeletal muscle mass. In fact, resistance training and NMES have even been applied successfully during acute exacerbations of COPD (13-16).

Recently, Probst and colleagues have demonstrated a significantly higher metabolic response, defined as oxygen uptake and minute ventilation, during high-intensity endurance training (cycling, walking and stair climbing) in comparison with highintensity leg press resistance training in COPD (17). At present, it remains unknown whether and to what extent the metabolic response during NMES is different from the metabolic response during resistance training in COPD. Hence, the purpose of this study was to compare the metabolic response during resistance training and during NMES of the quadriceps femoris muscles in patients with COPD entering pulmonary rehabilitation.

\section{MATERIALS AND METHODS}

This was a cross-sectional, comparative pilot study. A physiotherapist (MJHS) recruited the patients in the beginning of an inpatient pulmonary rehabilitation program at the Centre for Integrated Rehabilitation Organ failure (CIRO) in Horn, the Netherlands as described previously (18). Thirteen elderly patients (seven men) with mild to very severe COPD comprised the study group. All patients were treated with two or more of the following respiratory medications: long-acting $\beta 2$-sympathicomimeticum $(n=3)$, selective leukotriene antagonist $(n=1)$, long-acting parasympathicolyticum $(n=8)$, 
combination of long-acting $\beta 2$-sympathicomimeticum with inhalation corticosteroids $(n=8)$, theofylline $(n=3)$, inhalation corticosteroids $(n=3)$, mucolyticum $(n=6)$, $\beta$-sympathicomimeticum $(n=8)$, a combination of parasympathicolyticum with $\beta 2$-sympathicomimeticum ( $n=1)$, parasympathicolyticum $(n=3)$, and oral glucocorticosteroids ( $n=2$, both $5 \mathrm{mg} /$ day as maintenance therapy).

All procedures were in accordance with the recommendations found in the World Medical Association declaration of Helsinki (19). All patients gave informed consent to take part in the study.

Pulmonary function, body composition (overnight fasting bioelectrical impedance), peak aerobic capacity, peak cycling load, Borg symptom scores for dyspnea and fatigue (symptom-limited incremental cycle ergometer test), the Medical Research Council dyspnea grade, and the one-repetition maximum strength (1-RM) have been determined as described previously $(1,12)$.

In addition, all patients had to undergo one session of resistance training of the quadriceps femoris muscles and one session of NMES of the quadriceps femoris muscles on two separate days, in random order within the same week, after they were familiarized with the resistance training and NMES.

The resistance training consisted of a bilateral leg extension exercise. The patients were asked to perform three sets of repetitions at a load corresponding with $70 \%$ of the predetermined 1-RM (Technogym SpA, Gambettola, Italy). The patients had to rest for two minutes between every session of eight repetitions.

During the NMES, the quadriceps femoris muscles of both legs were electrically stimulated with a portable electrical stimulator (Gymnex 4, Gymna Uniphy N.V., Bilzen, Belgium). A total of eight electrodes were placed on the quadriceps femoris muscles (four on each leg): two on the vastus medialis and two on the rectus femoris muscles and the vastus lateralis muscle. The stimulation protocol consisted of a symmetrical biphasic square pulse at 75 Hertz, a duty cycle of 6 seconds on and 29 seconds off, a pulse time of $410 \mu \mathrm{s}$, intensity adjusted to individual toleration during a session lasting 21 minutes.

The metabolic response during resistance training and during NMES (e.g. peak aerobic capacity, $\mathrm{VO}_{2}$; and peak minute ventilation, $\mathrm{VE}$ ) was determined using an Oxycon Mobile (CareFusion, San Diego, Ca, USA). The methodology used to assess the metabolic response has been described in detail elsewhere (17). In addition, Borg symptom scores for dyspnea and leg fatigue (10 point scale) were obtained before and after both training sessions.

Because the data were not normally distributed, results are presented as median and interquartile range (IQR). For the same reason the Wilcoxon Signed Ranks Test was used to determine differences in $\mathrm{VO}_{2}, \mathrm{VE}$, and Borg symptom scores between resistance training and NMES. A priori, the level of statistical significance was set at $p \leq 0.05$. 


\section{RESULTS}

On average, patients had mild to severe COPD (GOLD II: $n=6$, GOLD III: $n=5$ and GOLD IV: $n=2$ ), an abnormal low body mass index, an abnormal low fat-free mass index, poor peak exercise capacity, and severe sensations of dyspnea during daily life (Table 4.1). None of the patients used supplemental oxygen at rest or during exercise.

The median absolute and relative peak $\mathrm{VO}_{2}$ during the resistance training session were significantly higher compared to the NMES session. In addition, median absolute and relative peak VE were significantly higher during the resistance training session compared to the NMES session. These higher metabolic responses were accompanied by higher symptom Borg scores for dyspnea and leg fatigue (Table 4.2). No serious adverse events occurred during both training sessions.

Table 4.1 Participant characteristics.

\begin{tabular}{|c|c|c|c|}
\hline & & Median & Interquartile range \\
\hline Age & years & 65 & $55-75$ \\
\hline Body weight & $\mathrm{kg}$ & 52.5 & $49-66$ \\
\hline BMI & $\mathrm{kg} / \mathrm{m}^{2}$ & 18.7 & $16.9-23.6$ \\
\hline FFMI & $\mathrm{kg} / \mathrm{m}^{2}$ & 13.7 & $13.3-14.9$ \\
\hline $\mathrm{FEV}_{1}$ & $\%$ pred & 49 & $33-57$ \\
\hline $\mathrm{FEV}_{1} / \mathrm{VC}$ & $\%$ pred & 53 & $40-65$ \\
\hline $\mathrm{DL}_{\mathrm{CO}}$ & $\%$ pred & 47.5 & $36-64$ \\
\hline MRC dyspnea & grade & 4 & $3-5$ \\
\hline Peak load CPET & watts & 49 & $35-74$ \\
\hline Peak load CPET & $\%$ pred & 42 & $31-67$ \\
\hline Peak VO ${ }_{2}$ CPET & $\mathrm{ml} / \mathrm{min}$ & 775 & $586-1112$ \\
\hline Peak VO ${ }_{2}$ CPET & $\%$ pred & 50 & $36-70$ \\
\hline Peak VE CPET & liters & 40 & $28-46$ \\
\hline Peak VE CPET & $\% \mathrm{MVV}$ & 85 & $62-104$ \\
\hline Peak HR CPET & bpm & 127 & $112-133$ \\
\hline Peak HR CPET & $\%$ pred & 77 & $75-85$ \\
\hline Borg dyspnea CPET & points & 7 & $4-8$ \\
\hline Borg fatigue CPET & points & 7 & $3-8$ \\
\hline
\end{tabular}

$\mathrm{BMI}=$ body mass index; $\mathrm{FFMI}=$ fat-free mass index; $\mathrm{FEV}_{1}=$ forced expiratory volume in one second; $\mathrm{VC}=\mathrm{vital}$ capacity; $\mathrm{MRC}=$ medical research council dyspnea scale; peak load=maximum workload; peak $\mathrm{VO}_{2}=$ peak oxygen uptake in $\mathrm{ml} / \mathrm{min}$; ; peak $\mathrm{VE}=$ peak minute ventilation in liters; peak $\mathrm{HR}=$ peak heart rate; bpm=beats per minute; \% pred=percentage predicted value; \% MVV=percentage maximal voluntary ventilation; CPET=cardiopulmonary exercise test. 
Table 4.2 Resistance training (RT) versus NMES.

\begin{tabular}{|c|c|c|c|c|}
\hline & & RT & NMES & p-value \\
\hline Resting $\mathrm{VO}_{2}$ & $\mathrm{ml} / \mathrm{min}$ & $273(229-311)$ & $241(208-283)$ & 0.099 \\
\hline Peak VO & $\mathrm{ml} / \mathrm{min}$ & 497 (443-592) & 311 (238-359) & 0.001 \\
\hline Peak VO & $\%$ peak $\mathrm{VO}_{2} \mathrm{CPET}$ & $57(45-84)$ & $34(30-42)$ & 0.001 \\
\hline Resting VE & liters & $14(12-18.5)$ & $13(11.5-15)$ & 0.023 \\
\hline Peak VE & liters & $28(22.0-32.5)$ & $14(12.5-19.5)$ & 0.001 \\
\hline Peak VE & $\%$ MVV & $58(43-78)$ & $31(25-37)$ & 0.001 \\
\hline Borg dyspnea & points & $3(2-4)$ & $1(1-3)$ & 0.005 \\
\hline Borg fatigue & points & $3(2.5-5)$ & $2(0.8-3.5)$ & 0.031 \\
\hline
\end{tabular}

The data shown (median (IQR)) are the results obtained before and after a session of resistance training (RT) or neuromuscular electrical (NMES) in 13 COPD patients. $\mathrm{VO}_{2}=$ oxygen uptake in $\mathrm{ml} / \mathrm{min}$; $\%$ peak $\mathrm{VO}_{2}$ $\mathrm{CPET}=$ oxygen uptake expressed as a percentage of the peak $\mathrm{VO}_{2}$ obtained at the end of a symptom-limited cardiopulmonary exercise test; peak $\mathrm{VE}=$ peak minute ventilation in liters; \% MVV=percentage maximal voluntary ventilation.

\section{DISCUSSION}

The present pilot study is the first to observe a higher metabolic response, perhaps reflecting higher metabolic demand during a resistance training session than during a NMES session in patients with COPD, accompanied by statistically significant higher Borg symptom scores for dyspnea and leg fatigue (Table 4.2).

Resistance training and NMES are two relatively new exercise modalities used in the rehabilitation of (severely) disabled patients with COPD. For example, 8 to 12 weeks of moderate to high intensity resistance training has been shown to improve skeletal muscle function, exercise capacity, and disease-specific health status in COPD patients with explicit quadriceps femoris muscle weakness $(20,21)$. Moreover, 4 to 6 weeks NMES of the quadriceps femoris muscles resulted in improved muscle function, exercise capacity, and disease-specific quality of life among COPD patients who had an abnormal body composition or who were too dyspneic to leave their home, respectively $(10,21)$. Finally, NMES have even resulted in faster mobilization from bed to chair in bed-bound COPD patients requiring prolonged mechanical ventilation (16). Based on the abovementioned findings, it seems reasonable to conclude that both modalities may be beneficial for COPD patients. Indeed, the findings of this study provide an additional rationale to apply both modalities in COPD patients who are severely disabled by their dyspnea. In the present study, median peak $\mathrm{VO}_{2}$ and median peak VE were low to moderate during a NMES session or a resistance training session, respectively, compared to peak $\mathrm{VO}_{2}$ and peak $\mathrm{VE}$ obtained during a symptom-limited incremental cycle ergometer test (Table 4.2). Probst and colleagues have found comparable results for a resistance training session in COPD patients during outpatient 
rehabilitation (14). Indeed, the metabolic response during endurance exercises has been shown to be significantly higher than resistance training in COPD (17).

Also, both NMES and resistance training appear to be applicable during acute COPD exacerbations, which are known to be associated with decline in functional status $(13,15)$.

Both resistance training and NMES are well tolerated and lead to acceptable levels of dyspnea and fatigue in COPD (Table 4.2). In this study, both training sessions were completed by all volunteering participants and did not result in serious adverse events or complaints of muscle soreness or dyspnea. Indeed, the biphasic current, which has been shown to produce high values for maximally electrically induced strength was well tolerated (22). Despite known benefits of both resistance training and NMES in COPD, the present authors do believe, however, that NMES has several major advantages compared to moderate to high intensity resistance training. First, NMES is feasible in the patients' home environment (10). Second, NMES is also applicable in COPD patients who suffer from long-lasting respiratory failure and who are bed-bound (16). Third, this study is the first to show that quadriceps femoris muscle training using NMES resulted in statistically significantly lower peak $\mathrm{VO}_{2}$ and peak $\mathrm{VE}$, associated with lower sensations of dyspnea and fatigue than a resistance training session. As such, patients with COPD whose ventilatory limitation or dyspnea precludes their participation in endurance or resistance type exercise training may better tolerate NMES.

This study had some methodological limitations and selected patient characteristics which may limit the external validity and broad applicability of the present findings: 1) only patients without long-term oxygen therapy were eligible due to the methodology used (17); and 2) on average patients had an abnormal body composition. Also, this study included a small number of patients with a wide range of COPD severity, and evaluated metabolic responses to NMES and resistance training during only a single session of each. Therefore, it is not possible to draw conclusions as yet regarding the relative metabolic responses over longer periods of training or to identify subpopulations of patients who may benefit particularly from either technique.

In conclusion, peak $\mathrm{VO}_{2}$, peak $\mathrm{VE}$, and symptoms of dyspnea and fatigue were significantly lower during a NMES session compared to a resistance training session among patients with COPD. Nevertheless, both modalities seem to result in an acceptable metabolic response accompanied by a clinically acceptable sensation of dyspnea and leg fatigue. These findings provide an additional rationale to design extra studies to assess the effects of NMES and resistance training in (severely) disabled patients with COPD. 


\section{REFERENCES}

1. Spruit MA, Pennings HJ, Janssen PP, Does JD, Scroyen S, Akkermans MA, et al. Extra-pulmonary features in COPD patients entering rehabilitation after stratification for MRC dyspnea grade. Respir Med. 2007;101(12):2454-63.

2. O'Donnell DE, McGuire M, Samis L, Webb KA. General exercise training improves ventilatory and peripheral muscle strength and endurance in chronic airflow limitation. Am J Respir Crit Care Med. 1998;157(5 Pt 1):1489-97.

3. Spruit MA, Troosters T, Trappenburg JC, Decramer M, Gosselink R. Exercise training during rehabilitation of patients with COPD: a current perspective. Patient Educ Couns. 2004;52(3):243-8.

4. Maltais F, LeBlanc P, Jobin J, Berube C, Bruneau J, Carrier L, et al. Intensity of training and physiologic adaptation in patients with chronic obstructive pulmonary disease. Am J Respir Crit Care Med. 1997; 155(2):555-61.

5. Sabapathy S, Kingsley RA, Schneider DA, Adams L, Morris NR. Continuous and intermittent exercise responses in individuals with chronic obstructive pulmonary disease. Thorax. 2004;59(12):1026-31.

6. Baarends EM, Schols AM, Mostert R, Wouters EF. Peak exercise response in relation to tissue depletion in patients with chronic obstructive pulmonary disease. Eur Respir J. 1997;10(12):2807-13.

7. Gosselink R, Troosters T, Decramer M. Peripheral muscle weakness contributes to exercise limitation in COPD. Am J Respir Crit Care Med. 1996;153(3):976-80.

8. Spruit MA, Wouters EF. New modalities of pulmonary rehabilitation in patients with chronic obstructive pulmonary disease. Sports Med. 2007;37(6):501-18.

9. Bourjeily-Habr G, Rochester CL, Palermo F, Snyder P, Mohsenin V. Randomised controlled trial of transcutaneous electrical muscle stimulation of the lower extremities in patients with chronic obstructive pulmonary disease. Thorax. 2002;57(12):1045-9.

10. Neder JA, Sword D, Ward SA, Mackay E, Cochrane LM, Clark CJ. Home based neuromuscular electrical stimulation as a new rehabilitative strategy for severely disabled patients with chronic obstructive pulmonary disease (COPD). Thorax. 2002;57(4):333-7.

11. Phillips $\mathrm{WT}$, Benton MJ, Wagner $\mathrm{CL}$, Riley $\mathrm{C}$. The effect of single set resistance training on strength and functional fitness in pulmonary rehabilitation patients. J Cardiopulm Rehabil. 2006;26(5):330-7.

12. Spruit MA, Gosselink R, Troosters T, De Paepe K, Decramer M. Resistance versus endurance training in patients with COPD and peripheral muscle weakness. Eur Respir J. 2002;19(6):1072-8.

13. Pitta F, Troosters T, Probst VS, Spruit MA, Decramer M, Gosselink R. Physical activity and hospitalization for exacerbation of COPD. Chest. 2006;129(3):536-44.

14. Probst VS, Troosters T, Celis G, Pitta F, Decramer M, Gosselink R. Effects of resistance exercise training during hospitalisation due to acute exacerbations of COPD-preliminary results. Eur Respir J Suppl. 2005; 26:432s.

15. Spruit MA, Gosselink R, Troosters T, Kasran A, Gayan-Ramirez G, Bogaerts P, et al. Muscle force during an acute exacerbation in hospitalised patients with COPD and its relationship with CXCL8 and IGF-I. Thorax. 2003;58(9):752-6.

16. Zanotti E, Felicetti G, Maini M, Fracchia C. Peripheral muscle strength training in bed-bound patients with COPD receiving mechanical ventilation: effect of electrical stimulation. Chest. 2003;124(1):292-6.

17. Probst VS, Troosters T, Pitta F, Decramer M, Gosselink R. Cardiopulmonary stress during exercise training in patients with COPD. Eur Respir J. 2006;27(6):1110-8.

18. Franssen FM, Broekhuizen R, Janssen PP, Wouters EF, Schols AM. Effects of whole-body exercise training on body composition and functional capacity in normal-weight patients with COPD. Chest. 2004;125(6):2021-8.

19. World Medical Association declaration of Helsinki. Recommendations guiding physicians in biomedical research involving human subjects. J Am Med Assoc. 1997;277(11):925-6.

20. Simpson K, Killian K, McCartney N, Stubbing DG, Jones NL. Randomised controlled trial of weightlifting exercise in patients with chronic airflow limitation. Thorax. 1992;47(2):70-5.

21. Vivodtzev I, Pepin JL, Vottero G, Mayer V, Porsin B, Levy P, et al. Improvement in quadriceps strength and dyspnea in daily tasks after 1 month of electrical stimulation in severely deconditioned and malnourished COPD. Chest. 2006;129(6):1540-8. 
22. Crevenna R, Posch M, Sochor A, Keilani M, Wiesinger G, Nuhr M, et al. [Optimizing electrotherapy--a comparative study of 3 different currents]. Wien Klin Wochenschr. 2002;114(10-11):400-4. Optimierung der Schwellstrombehandlung--eine Vergleichsstudie von drei unterschiedlichen Stromformen. 



\section{CHAPTER 5}

Oxygen uptake, ventilation, and symptoms during

low-frequency versus high-frequency NMES in COPD:

a pilot study

Maurice JH Sillen, Emiel FM Wouters, Frits ME Franssen, Kenneth Meijer,

Koen HP Stakenborg, Martijn A Spruit

Lung 2011;189:21-26 


\section{ABSTRACT}

\section{Background}

Transcutaneous neuromuscular electrical stimulation (NMES) involves the application of an electrical current through electrodes placed on the skin over the targeted muscles. During high-frequency NMES (HF-NMES), oxygen uptake, minute ventilation, and the degree of symptom perception (dyspnea and fatigue) have been shown to be acceptable in chronic obstructive pulmonary disease (COPD). Currently, oxygen uptake and ventilation load have never been assessed during low-frequency NMES (LF-NMES) of the lower-limb muscles. The purpose of this study was to compare prospectively oxygen uptake, ventilation, and symptom perception during a single session of LF-NMES versus a single session of HF-NMES of quadriceps muscles in patients with COPD.

\section{Methods}

In 17 COPD patients (mean $\mathrm{FEV}_{1}$ : $45 \%$ predicted, mean body mass index: $26.2 \mathrm{~kg} / \mathrm{m}^{2}$ ), peak exercise capacity, functional exercise capacity and the Medical Research Council dyspnea grade were evaluated. In addition, oxygen uptake, minute ventilation, heart rate, and Borg symptom scores were assessed during one session of LF-NMES (15 Hertz) and one session of HF-NMES (75 Hertz) and compared with peak values.

\section{Results}

Mean oxygen uptake (LF-NMES: $327 \mathrm{ml} / \mathrm{min}$ versus HF-NMES: $315 \mathrm{ml} / \mathrm{min}$ ), minute ventilation (LF-NMES: $14 \mathrm{~L}$ versus HF-NMES: $15 \mathrm{~L}$ ), and heart rate (LF-NMES: 86 BPM versus HF-NMES: 83 BPM) were similar during both NMES frequencies. Patients used a relatively low proportion of their peak aerobic capacity during both NMES sessions (LF-NMES: $34 \%$ versus HF-NMES: 33\%; $p=0.397)$. In addition, symptom Borg scores for dyspnoea and leg fatigue were also comparable.

\section{Conclusions}

Oxygen uptake, ventilation, and symptoms of dyspnea and fatigue were comparable and tolerable during LF-NMES and HF-NMES in patients with COPD. Therefore, LF-NMES and HF-NMES may both be suitable rehabilitative modalities to be used in severely dyspneic patients with lower-limb muscle dysfunction. 


\section{INTRODUCTION}

Lower-limb skeletal muscle dysfunction (e.g. quadriceps weakness and/or loss of quadriceps muscle endurance) is a common extrapulmonary manifestation of chronic obstructive pulmonary disease (COPD) (1-3) and contributes to a poor exercise performance, increased use of health care and mortality in COPD patients (4-6). Daily physical inactivity partially determines skeletal muscle dysfunction in COPD (7). Therefore, these patients have a clear indication for pulmonary rehabilitation $(8,9)$.

Exercise-based rehabilitation programs are able to improve lower-limb muscle strength and endurance, functional exercise performance, and health status in patients with COPD (10,11). Exercise training programs typically consist of lower-limb endurance/interval training, resistance training or a combination thereof $(12,13)$. Unfortunately, the benefits of these lower-limb endurance exercises (e.g. treadmill walking and/or stationary ergometry cycling) may be limited in COPD patients due to exercise-induced dyspnea and ventilatory limitation during training (14). Indeed, aerobic exercise training can result in high values of oxygen uptake and ventilation, which exact a relatively high claim on the peak aerobic capacity of patients with COPD (14). Therefore, there is great interest in new and effective alternate rehabilitative modalities that do not evoke dyspnea, such as high-intensity resistance training and transcutaneous neuromuscular electrical stimulation (NMES) $(14,15)$.

NMES involves the application of an electrical current through electrodes placed on the skin over the targeted muscles, thereby depolarizing motor endplates and, in turn, inducing skeletal muscle contractions $(16,17)$. In patients with chronic heart failure lowfrequency NMES (LF-NMES, $<50$ Hertz) increased muscle endurance (18), whereas highfrequency NMES (HF-NMES, $\geq 50$ Hertz) increased muscle strength (19). To date, it remains unknown which stimulation frequency leads to better gains in muscle strength and endurance and which stimulation protocol is optimal for patients with COPD. Indeed, the effects of LF-NMES are unknown in COPD. Nevertheless, due to the striking similarities in systemic factors that contribute to exercise intolerance in patients with COPD or chronic heart failure (20), a reduced fiber type I proportion (21), and loss of muscle endurance in COPD $(1,22)$, it seems reasonable to hypothesize that LF-NMES will also have positive effects on quadriceps muscle endurance and functional exercise capacity in COPD.

Recently, oxygen uptake, minute ventilation, and the degree of symptom perception (dyspnea and fatigue) have been shown to be acceptable during a single session of HFNMES in patients with COPD (15). During LF-NMES, the authors expect also a relatively low and acceptable oxygen uptake and ventilation during LF-NMES, which is comparable to HF-NMES (15). Currently, this has never been studied but seems a 
necessary step prior to a randomized controlled trial in which the effects of NMES (LF versus HF) on lower-limb muscle dysfunction will be assessed in COPD.

The purpose of this study was to compare prospectively oxygen uptake, ventilation and symptom perception during a single session of LF-NMES and a single session of HFNMES of the quadriceps muscles in patients with COPD. In addition, safety was assessed by monitoring adverse events.

\section{METHODS}

\section{Study subjects}

Seventeen patients (10 men) with clinically stable COPD (23) were recruited the start of a comprehensive pulmonary rehabilitation program at Ciro in Horn (the Netherlands) (9). Patients were considered ineligible to participate if they had neuromuscular disorders, metal implants in the lower limbs, a cardiac pacemaker, and/or an exacerbation of symptoms in the preceding 4 weeks. Patients with long-term oxygen therapy were excluded because of the methodology that is used to measure oxygen uptake and ventilation (e.g., Oxycon Mobile).

\section{Study Design}

This was a prospective, randomized crossover pilot study. The study protocol was approved by the Medical Ethical Committee of the Maastricht University Medical Centre+ (MEC 09-3-004) and conformed to the principles outlined in the World Medical Association declaration of Helsinki which is revised in Seoul. Details of the trial were registered at http://www.trialregister.nl (NTR1834) before subject enrolment. All patients gave written informed consent to take part in the study.

Pulmonary function, body composition (whole-body dual-energy X-ray absorptiometry), peak cycling load, peak aerobic capacity, functional exercise capacity, quadriceps muscle strength, Borg symptom scores for dyspnea and fatigue, and the Medical Research Council dyspnea grade were determined at baseline, as a routine part of entry into the pulmonary rehabilitation program $(9,24)$. Coexisting morbidities were assessed using the Charlson comorbidity index (CCI) (25). Moreover, all patients underwent a single session of LF-NMES and a single session of HF-NMES on two separate days within the same week, in random order. These sessions occurred during the first week of the rehabilitation program, 0-4 weeks after the baseline testing. Randomization was performed by means of a computer generated randomization list. 
Safety was assessed by monitoring adverse events. Adverse events were defined as changes in health or side-effects, such as pain and/or muscle cramps, that occurred in patients who participated in the study while receiving the treatment.

\section{Methods}

During both NMES sessions, continuous on-line calculations of breath-by-breath oxygen uptake $\left(\mathrm{VO}_{2}\right)$ and minute ventilation (VE) were obtained using the Oxycon Mobile, a portable metabolic system (CareFusion, San Diego, Ca, USA) (Figure 5.1). After calibration the face mask (Combitox, Dräger Safety, Lübeck, Germany) was carefully adjusted to the patient's face and checked for air leaks. Data were collected breath by breath and processed using JLAB version $5.20 \mathrm{~b}$ software (CareFusion).

\section{NMES PROTOCOLS}

During both NMES sessions, bilateral electrical stimulation of the quadriceps muscles was applied using a portable electrical stimulator (Gymnex 4, Gymna Uniphy N.V., Bilzen, Belgium). A total of eight electrodes were placed on the quadriceps femoris muscles (four on each leg): two on the vastus medialis, one on the rectus femoris muscle and one on the vastus lateralis muscle (Figure 5.1). Both stimulation protocols were preset protocols in the devices. The stimulation protocol of LF-NMES consisted of a symmetrical biphasic square pulse at $15 \mathrm{Hertz}$, a duty cycle of 8 seconds on and 2 seconds off, a pulse time of $390 \mu$ s during a session lasting 29 minutes. The stimulation protocol of the HF-NMES consisted of a symmetrical biphasic square pulse at 75 Hertz, a duty cycle of 6 seconds on and 29 seconds off, a pulse time of $410 \mu \mathrm{s}$ during a session lasting 21 minutes. During both sessions, the intensity was increased to maximal individual toleration. The muscle contractions were visible and palpable. In addition, Borg symptom scores for dyspnea and leg fatigue were obtained before and after both NMES sessions (26).

\section{Statistical ANALYSES}

All statistical analyses were performed using SPSS for Windows, Version 17.0.1 (SPSS, Inc., Chicago, II, USA). Continuous data were tested for normality and presented as mean and standard deviation or as median and interquartile range (IQR). In addition, two-tailed paired t-tests or Wilcoxon Signed Ranks Tests were used for within-group comparisons. A priori, the level of statistical significance was set at $p \leq 0.05$. 
Figure 5.1 Measurement of oxygen uptake and minute ventilation during a session of bilateral NMES of the quadriceps muscles in a male patient with COPD.

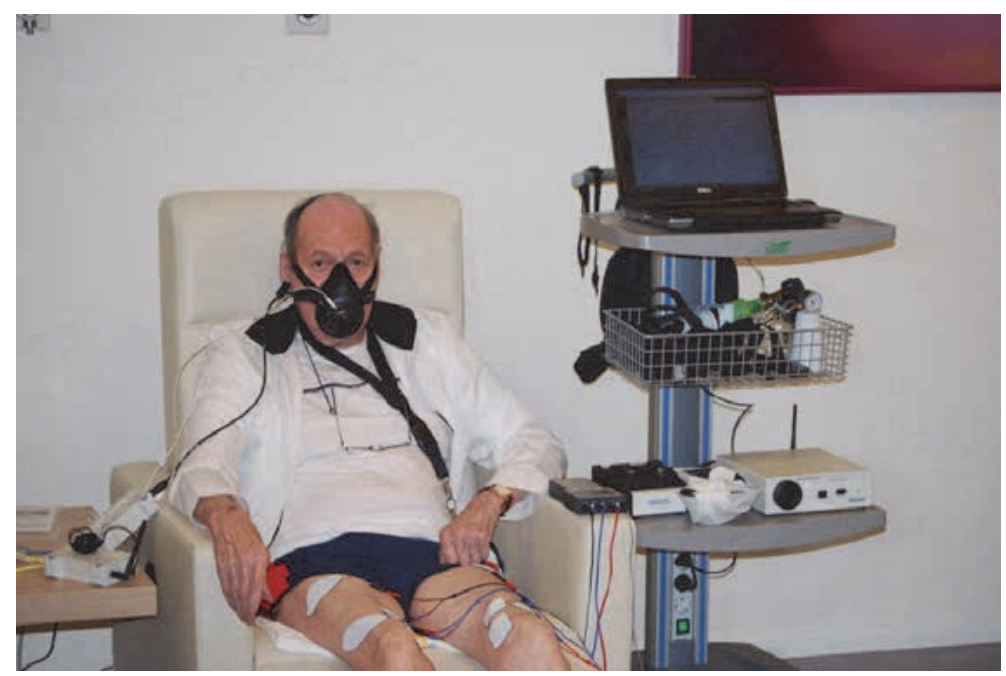

\section{RESULTS}

\section{Characteristics}

The baseline characteristics of the patients are given in Table 5.1. On average, patients had moderate to severe COPD and a poor diffusing capacity of the lung for carbon monoxide (Table 5.1). Most patients were severely dyspneic, reporting on the MRC dyspnea scale that they had to stop for breath after walking 100 yards (91.4 meters) or after a few minutes on the level. Moreover, patients had explicit quadriceps weakness (mean \pm SD: $55 \pm 12 \%$ predicted (217)), as well as poor functional and peak exercise performance, with a mean $\pm S D$ 6-minute walk distance of $380 \pm 98$ meters (218), $\mathrm{VO}_{2}$ max and maximal workload during CPET of $986 \pm 260 \mathrm{ml} / \mathrm{min}$ and $60 \pm 21$ Watts, respectively. On average, patients were ventilatory limited at the end of the cardiopulmonary exercise test accompanied with high Borg scores for dyspnea and fatigue (Table 5.1). 
Table 5.1 Participant characteristics.

\begin{tabular}{|c|c|c|}
\hline Age & years & $67 \pm 9$ \\
\hline Body weight & $\mathrm{kg}$ & $72.7 \pm 14.7$ \\
\hline BMI & $\mathrm{kg} / \mathrm{m}^{2}$ & $26.2 \pm 4.5$ \\
\hline FFMI & $\mathrm{kg} / \mathrm{m}^{2}$ & $17.5 \pm 2.4$ \\
\hline $\mathrm{FEV}_{1}$ & $\%$ predicted & $45 \pm 16$ \\
\hline $\mathrm{FEV}_{1} / \mathrm{VC}$ max & $\%$ & $35 \pm 8$ \\
\hline $\mathrm{DL}_{\mathrm{co}}$ & $\%$ predicted & $57 \pm 22$ \\
\hline MRC dyspnea & Grade & $4 \pm 1$ \\
\hline Peak torque quadriceps muscles & $\%$ predicted & $55 \pm 12$ \\
\hline 6MWD & meters & $380 \pm 98$ \\
\hline 6MWD & $\%$ predicted & $63 \pm 17$ \\
\hline Peak load CPET & Watts & $60 \pm 21$ \\
\hline Peak load CPET & $\%$ predicted & $52 \pm 26$ \\
\hline Peak VO ${ }_{2} \mathrm{CPET}$ & $\mathrm{ml} / \mathrm{min}$ & $986 \pm 260$ \\
\hline Peak VO ${ }_{2} \mathrm{CPET}$ & $\%$ predicted & $66 \pm 30$ \\
\hline Peak VCO ${ }_{2}$ CPET & $\mathrm{ml} / \mathrm{min}$ & $945 \pm 281$ \\
\hline Peak VE CPET & liters & $38 \pm 9$ \\
\hline Peak VE CPET & $\% \mathrm{MVV}$ & $93 \pm 23$ \\
\hline Peak HR CPET & BPM & $119 \pm 15$ \\
\hline Peak HR CPET & $\%$ predicted & $78 \pm 8$ \\
\hline Borg dyspnea CPET & points & $8 \pm 2$ \\
\hline Borg fatigue CPET & points & $6 \pm 2$ \\
\hline
\end{tabular}

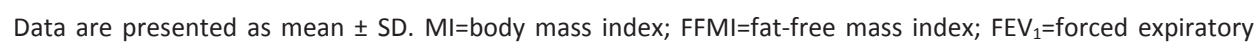
volume in one second; $V C$ max=maximum vital capacity; $\mathrm{DL}_{\mathrm{co}}=$ diffusion capacity of the lung for carbon monoxide; $\mathrm{MRC}=$ medical research council dyspnea scale; $6 \mathrm{MWD}=$ distance achieved by the six-minute walking test; peak load=maximum workload; $\mathrm{CPET}=$ cardiopulmonary exercise test; peak $\mathrm{VO}_{2}=$ peak oxygen uptake in $\mathrm{ml} / \mathrm{min}$.; peak $\mathrm{VE}=$ peak minute ventilation in liters; peak $\mathrm{HR}=$ peak heart rate; $\mathrm{bpm}=$ beats per minute; $\%$ pred=percentage predicted value; $\% \mathrm{MVV}=$ percentage maximal voluntary ventilation

Significant comorbidities were present in 10 out of 17 (59\%) patients with COPD. Indeed, the median score on the Charlson comorbidity index was 2 (IQR: 1-2.5) points. These comorbidities were: coronary heart disease $(n=5)$, diabetes $(n=3)$, non-metastatic solid malignancy $(n=2)$, peripheral artery disease $(n=2)$, chronic heart failure $(n=1)$, transient ischemic attack $(n=1)$, Bechterew's disease $(n=1)$ and/or peptic ulcer $(n=1)$. The following pulmonary maintenance medications were used by 17 COPD patients who volunteered to participate: short-acting $\beta_{2}$-agonists $(n=8)$, short-acting anticholinergics $(n=1)$, short-acting combined bronchodilators $(n=7)$, long-acting $\beta_{2^{-}}$ agonists $(\operatorname{LABA})(n=3)$, long-acting anticholinergics $(n=15)$, inhaled glucocorticosteroids (alone or in combination with LABA) $(n=16)$, systemic glucocorticosteroids $(n=2)$, xanthine derivatives $(n=3)$, antioxidant agents $(n=5)$, and/or leucotriene modifiers $(n=1)$. 


\section{LF-NMES VERSUS HF-NMES}

All patients were able to complete both NMES sessions. No differences in tolerating both frequencies were reported and no adverse events occurred during either type of training session.

The effects of LF-NMES and HF-NMES are compared in Table 5.2. Mean resting and peak oxygen uptake, minute ventilation, and heart rate were not significantly different between LF-NMES and HF-NMES sessions (Table 5.2). Indeed, patients used a relatively low proportion of their peak aerobic capacity measured previously during a CPET, during both NMES sessions (LF-NMES: 34\%; HF-NMES: 33\%; $p=0.386$ ). Also, Borg symptom scores for dyspnea and leg fatigue were comparable for both NMES types (Table 5.2).

Table 5.2 Low-frequency NMES versus high-frequency NMES in COPD.

\begin{tabular}{llccc}
\hline & & $\begin{array}{c}\text { Low-frequency } \\
\text { NMES }\end{array}$ & $\begin{array}{c}\text { High-frequency } \\
\text { NMES }\end{array}$ & p-value \\
\hline Resting VO & ml/min & $258 \pm 65$ & $238 \pm 51$ & 0.178 \\
Peak VO $_{2}$ & $\mathrm{ml} / \mathrm{min}$ & $327 \pm 96$ & $315 \pm 84$ & 0.538 \\
Peak VO & \% peak VO ${ }_{2} \mathrm{CPET}$ & $34 \pm 12$ & $33 \pm 12$ & 0.386 \\
Resting VE & liters & $11 \pm 2$ & $11 \pm 2$ & 0.633 \\
Peak VE & liters & $14 \pm 4$ & $15 \pm 4$ & 0.582 \\
Peak VE & $\% \mathrm{MVV}$ & $36 \pm 13$ & $38 \pm 15$ & 0.249 \\
Peak HR & BPM & $86 \pm 13$ & $83 \pm 11$ & 0.284 \\
Peak HR & $\% \mathrm{CPET}$ & $72 \pm 13$ & $71 \pm 11$ & 0.374 \\
Borg dyspnea & points & $2 \pm 1$ & $2 \pm 1$ & 0.154 \\
Borg fatigue & points & $2 \pm 2$ & $2 \pm 1$ & 0.680 \\
\hline
\end{tabular}

The data are presented as mean \pm SD before and after a single session of low-frequency neuromuscular electrical stimulation (LF-NMES) versus high-frequency neuromuscular electrical stimulation (HF-NMES) in 17 COPD patients. $\mathrm{VO}_{2}=$ oxygen uptake in $\mathrm{ml} / \mathrm{min}$; \% peak $\mathrm{VO}_{2} \mathrm{CPET}=$ oxygen uptake expressed as a proportion of the peak $\mathrm{VO}_{2}$ obtained at the end of a symptom-limited cardiopulmonary exercise test; peak $\mathrm{VE}=$ peak minute ventilation in liters; $\% \mathrm{MVV}=$ percentage maximal voluntary ventilation $\left(\mathrm{MVV}=40 * \mathrm{FEV}_{1}\right)$

\section{DISCUSSION}

The present pilot study is the first to assess oxygen uptake, ventilation, and symptom perception during a session of LF-NMES and a session of HF-NMES. Both NMES frequencies appear to be safe and sustainable in dyspneic COPD patients with comorbidities. Moreover, oxygen uptake, ventilation, and Borg symptom scores for dyspnea and leg fatigue were relatively low and comparable between LF-NMES and HF-NMES. 
HF-NMES is a relatively new exercise modality used in the rehabilitation of (severely) disabled patients with COPD $(17,29,30)$. For example, 4 to 6 weeks of HF-NMES of the quadriceps muscles resulted in improved muscle strength, exercise capacity and disease-specific quality of life in COPD patients who had an abnormal baseline body composition or who were too dyspneic to leave their home for a hospital-based outpatient pulmonary rehabilitation program (31,32). Indeed, HF-NMES has even resulted in faster mobilization from bed to chair in bed-bound COPD patients requiring prolonged mechanical ventilation (33). Therefore, it seems reasonable to conclude that HF-NMES is safe, feasible, and beneficial in patients with COPD (29). Indeed, international guidelines recommend HF-NMES in patients with severe chronic respiratory disease with extreme skeletal muscle weakness or who are bed-bound (8).

To date, the effects of LF-NMES have not been studied yet in COPD. Nevertheless, based on the combination of severe dyspnea at rest and a clear loss of lower-limb muscle endurance (1), it may be worthwhile to assess the effects of LF-NMES of muscle of ambulation in patients with COPD. The present findings provide an additional rationale to assess the effects of LF-NMES in patients with COPD who are severely disabled by their dyspnea. In fact, mean peak oxygen uptake, ventilation, and heart rate were well below maximum values during a session of LF-NMES and comparable to the values during a session of HF-NMES (Table 5.2).

Oxygen uptake, ventilation, and symptoms during a session of HF-NMES in the present study corroborates previous results of our group (15). Yet again, these findings show that COPD patients use a clearly lower proportion of their peak aerobic capacity during a session of NMES compared to a session of high-intensity lower-limb resistance training (15), treadmill walking or stationary cycling (14). Indeed, the biphasic current used during the HF-NMES and LF-NMES was well tolerated by the patients (e.g. no drop out from the protocol and no adverse events) and led to acceptable levels of dyspnea and fatigue in COPD (Table 5.2).

This pilot study had some methodological limitations and selected patient characteristics that may limit the external validity and broad applicability of the present findings. Only patients without long-term oxygen therapy were eligible due to the methodology used (14). This study included a small number of COPD patients with a wide range of disease severity and different levels of physical functioning. Some of the participants may not be the typical patients who receives NMES in daily clinical practice. Indeed, NMES can be particularly useful for severely disabled patients with COPD (29).

Moreover, oxygen uptake, and ventilation were evaluated during only a single session of LF-NMES and HF-NMES. Therefore, it is not possible to draw conclusions as yet regarding the relative values of oxygen uptake and ventilation over longer periods of 
training or to identify subpopulations of patients who may benefit particularly from either technique.

Session time, duty cycle, and pulse duration differed between both NMES protocols. This was due to preset protocols of the commercially available NMES device. Nevertheless, the impact of these NMES features on the primary outcome (i.e. oxygen uptake) is expected to be nil.

Finally, magnetic stimulation can also be used as a non-voluntarily lower-limb muscle training method in patients with COPD (34). However, magnetic stimulation is rather expensive and its clinical applicability in groups of COPD patients in clinical routine seems challenging.

In conclusion, oxygen uptake, ventilation, and symptom perception of dyspnea and leg fatigue were sustainable low and comparable during a single session of LF-NMES and HF-NMES in patients with COPD. These pilot findings provide an additional rationale to design randomized controlled trials to compare the effects of LF-NMES and HF-NMES on lower-limb dysfunction in (severely) disabled patients with COPD. 


\section{REFERENCES}

1. Coronell C, Orozco-Levi M, Mendez R, Ramirez-Sarmiento A, Galdiz JB, Gea J. Relevance of assessing quadriceps endurance in patients with COPD. Eur Respir J. 2004;24(1):129-36.

2. Seymour JM, Spruit MA, Hopkinson NS, Sathyapala SA, Man WD, Jackson A, et al. The prevalence of quadriceps weakness in COPD and the relationship with disease severity. Eur Respir J. 2009.

3. Franssen FM, Broekhuizen R, Janssen PP, Wouters EF, Schols AM. Limb muscle dysfunction in COPD: effects of muscle wasting and exercise training. Med Sci Sports Exerc. 2005;37(1):2-9.

4. Decramer M, Gosselink R, Troosters T, Verschueren M, Evers G. Muscle weakness is related to utilization of health care resources in COPD patients. Eur Respir J. 1997;10(2):417-23.

5. Gosselink R, Troosters T, Decramer M. Peripheral muscle weakness contributes to exercise limitation in COPD. Am J Respir Crit Care Med. 1996;153(3):976-80.

6. Swallow EB, Reyes D, Hopkinson NS, Man WD, Porcher R, Cetti EJ, et al. Quadriceps strength predicts mortality in patients with moderate to severe chronic obstructive pulmonary disease. Thorax. 2007;62(2):115-20.

7. ATS/ERS. Skeletal muscle dysfunction in chronic obstructive pulmonary disease. A statement of the American Thoracic Society and European Respiratory Society. Am J Respir Crit Care Med. 1999;159(4 Pt 2):S1-40.

8. Nici L, Donner C, Wouters E, Zuwallack R, Ambrosino N, Bourbeau J, et al. American Thoracic Society/European Respiratory Society statement on pulmonary rehabilitation. Am J Respir Crit Care Med. 2006;173(12):1390-413.

9. Spruit MA, Vanderhoven-Augustin I, Janssen PP, Wouters EF. Integration of pulmonary rehabilitation in COPD. Lancet. 2008;371(9606):12-3.

10. Lacasse Y, Goldstein R, Lasserson TJ, Martin S. Pulmonary rehabilitation for chronic obstructive pulmonary disease. Cochrane Database Syst Rev. 2006(4):CD003793.

11. Spruit MA, Gosselink R, Troosters T, De Paepe K, Decramer M. Resistance versus endurance training in patients with COPD and peripheral muscle weakness. Eur Respir J. 2002;19(6):1072-8.

12. Beauchamp MK, Dolmage TE, Nonoyama M, Goldstein RS, Hill K, Mathur S, et al. Interval versus continuous training in individuals with COPD - a systematic review. Thorax. 2009.

13. Spruit MA, Wouters EF. New modalities of pulmonary rehabilitation in patients with chronic obstructive pulmonary disease. Sports Med. 2007;37(6):501-18.

14. Probst VS, Troosters T, Pitta F, Decramer M, Gosselink R. Cardiopulmonary stress during exercise training in patients with COPD. Eur Respir J. 2006;27(6):1110-8.

15. Sillen MJ, Janssen PP, Akkermans MA, Wouters EF, Spruit MA. The metabolic response during resistance training and neuromuscular electrical stimulation (NMES) in patients with COPD, a pilot study. Respir Med. 2008;102(5):786-9.

16. Vanderthommen M, Crielaard JM. [Muscle electric stimulation in sports medicine]. Rev Med Liege. 2001;56(5):391-5. Electromyostimulation en medecine du sport.

17. Vivodtzev I, Lacasse $\mathrm{Y}$, Maltais F. Neuromuscular electrical stimulation of the lower limbs in patients with chronic obstructive pulmonary disease. J Cardiopulm Rehabil Prev. 2008;28(2):79-91.

18. Nuhr MJ, Pette D, Berger R, Quittan M, Crevenna R, Huelsman M, et al. Beneficial effects of chronic low-frequency stimulation of thigh muscles in patients with advanced chronic heart failure. Eur Heart J. 2004;25(2):136-43.

19. Quittan M, Wiesinger GF, Sturm B, Puig S, Mayr W, Sochor A, et al. Improvement of thigh muscles by neuromuscular electrical stimulation in patients with refractory heart failure: a single-blind, randomized, controlled trial. Am J Phys Med Rehabil. 2001;80(3):206-14; quiz 15-6, 24.

20. Gosker HR, Lencer NH, Franssen FM, van der Vusse GJ, Wouters EF, Schols AM. Striking similarities in systemic factors contributing to decreased exercise capacity in patients with severe chronic heart failure or COPD. Chest. 2003;123(5):1416-24.

21. Gosker HR, Zeegers MP, Wouters EF, Schols AM. Muscle fibre type shifting in the vastus lateralis of patients with COPD is associated with disease severity: a systematic review and meta-analysis. Thorax. 2007;62(11):944-9. 
22. Van 't Hul AHJ, Gosselink R, Hollander P, Postmus P, Kwakkel G. Quadriceps muscle endurance in patients with chronic obstructive pulmonary disease. Muscle and Nerve 2004;29(2):267-74.

23. Rabe KF, Hurd S, Anzueto A, Barnes PJ, Buist SA, Calverley P, et al. Global strategy for the diagnosis, management, and prevention of chronic obstructive pulmonary disease: GOLD executive summary. Am J Respir Crit Care Med. 2007;176(6):532-55.

24. Spruit MA, Pennings HJ, Janssen PP, Does JD, Scroyen S, Akkermans MA, et al. Extra-pulmonary features in COPD patients entering rehabilitation after stratification for MRC dyspnea grade. Respir Med. 2007;101(12):2454-63.

25. Charlson ME, Pompei P, Ales KL, MacKenzie CR. A new method of classifying prognostic comorbidity in longitudinal studies: development and validation. J Chronic Dis. 1987;40(5):373-83.

26. Borg GA. Psychophysical bases of perceived exertion. Med Sci Sports Exerc. 1982;14(5):377-81.

27. Borges $\mathrm{O}$. Isometric and isokinetic knee extension and flexion torque in men and women aged 20-70. Scand J Rehabil Med. 1989;21(1):45-53.

28. Troosters T, Gosselink R, Decramer M. Six minute walking distance in healthy elderly subjects. Eur Respir J. 1999;14(2):270-4.

29. Sillen MJ, Speksnijder CM, Eterman RM, Janssen PP, Wagers SS, Wouters EF, et al. Effects of neuromuscular electrical stimulation of muscles of ambulation in patients with chronic heart failure or COPD: a systematic review of the English-language literature. Chest. 2009;136(1):44-61. E

30. Roig M, Reid WD. Electrical stimulation and peripheral muscle function in COPD: A systematic review. Respir Med. 2008.

31. Neder JA, Sword D, Ward SA, Mackay E, Cochrane LM, Clark CJ. Home based neuromuscular electrical stimulation as a new rehabilitative strategy for severely disabled patients with chronic obstructive pulmonary disease (COPD). Thorax. 2002;57(4):333-7.

32. Vivodtzev I, Pepin JL, Vottero G, Mayer V, Porsin B, Levy P, et al. Improvement in quadriceps strength and dyspnea in daily tasks after 1 month of electrical stimulation in severely deconditioned and malnourished COPD. Chest. 2006;129(6):1540-8.

33. Zanotti E, Felicetti G, Maini M, Fracchia C. Peripheral muscle strength training in bed-bound patients with COPD receiving mechanical ventilation: effect of electrical stimulation. Chest. 2003;124(1):292-6.

34. Bustamante EL, Gorostiza A, Jimenez U, Galdiz JB. Muscle training with repetitive magnetic stimulation of the quadriceps in severe COPD patients. Respiratory Medicine. 2010;104(2):237-45. 


\section{CHAPTER 6}

\section{Heterogeneity in clinical characteristics and co- \\ morbidities in dyspneic individuals with COPD \\ GOLD D: Findings of the DICES trial}

Maurice JH Sillen, Frits ME Franssen, Jeannet ML Delbressine,

Nicole HMK Uszko-Lencer, Lowie EGW Vanfleteren, Erica PA Rutten, Emiel FM Wouters, Martijn A Spruit

Respiratory Medicine 2013;107:1186-1194 


\section{ABSTRACT}

\section{Introduction}

Chronic obstructive pulmonary disease (COPD) is a complex and heterogeneous respiratory disease with important extra-pulmonary features and comorbidities. The aim of this study was to assess clinical heterogeneity in a well-defined subgroup of individuals with COPD GOLD D, including possible gender differences.

\section{Methods}

Pulmonary function, arterial blood gases, exercise performance, quadriceps muscle function, problematic activities of daily life, dyspnea, health status and comorbidities have been assessed in 117 individuals with an MRC dyspnea grade 4/5 and COPD GOLD D entering pulmonary rehabilitation.

\section{Results}

A broad range of values were found for diffusion capacity, exercise capacity, quadriceps muscle function and health status. Indeed, high coefficients of variation were found for these outcomes. Problematic activities of daily life as well as objectified comorbidities also varied to a great extent. Moreover, significant gender differences were found for exercise performance, lower-limb muscle function and various comorbidities.

\section{Conclusion}

The current findings emphasize that COPD is a heterogeneous disease whose clinical presentation varies significantly, even in individuals with very severe COPD with the same degree of dyspnea and all classified as GOLD D. 


\section{INTRODUCTION}

Chronic obstructive pulmonary disease (COPD) is an important cause of morbidity, disability-adjusted life years and mortality worldwide (1). Generally, a significant heterogeneity in symptoms, clinical characteristics and comorbidities exist in patients with COPD (2-7). Besides the degree of airflow limitation, symptoms, exacerbations and comorbidities contribute to the overall severity in patients with COPD (7-9). The latest GOLD document stratifies patients into four groups (i.e., A to D) based on a combined assessment, including the degree of airflow limitation, future exacerbation risk and the burden of disease (9).

The Medical Research Council (MRC) scale is a simple, readily available office tool to grade dyspnea (10). In the latest GOLD document, the modified MRC has been used to determine the burden of disease (9). Generally, clinical characteristics and extrapulmonary features of COPD are different after stratification for MRC dyspnea grade (11-14). For example, COPD patients with MRC dyspnea grades $4 / 5$ have an increased risk for ischemic electrocardiographic changes (14), worse exercise performance, higher scores for anxiety and depression and worse disease-specific health status (12) as compared to those with lower MRC dyspnea grades. Moreover, during domestic activities of daily life COPD patients with MRC dyspnea grades 4 or 5 use a significantly higher proportion of their peak aerobic capacity and ventilation accompanied with higher task-related dyspnea scores (13). So, MRC dyspnea grades $4 / 5$ provide a rough indication of the clinical status of patients with COPD. However, anecdotal clinical experience suggests that COPD is a complex, heterogeneous disease whose clinical presentation varies significantly despite having the same degree of dyspnea. Moreover, multiple studies have found that women with COPD report higher (=worse) scores on the MRC dyspnea scale than men with COPD for the same degree of airflow limitation (15-17). This suggests that individuals with COPD GOLD D will have a heterogeneous clinical presentation, despite belonging to the same GOLD group.

Therefore, we sought to characterize the heterogeneity of individuals with COPD with GOLD D, including possible gender differences.

\section{METHODS}

\section{Patients AND setting}

Individuals with COPD who were referred by their chest physician for an interdisciplinary pulmonary rehabilitation program at CIRO+ were recruited (18). Individuals with COPD as the primary diagnosis (8) and an MRC dyspnea grade 4 or 5 were eligible (19). As these are the baseline findings of the DICES trial (Dyspneic 
Individuals with COPD: Electrical stimulation or Strength training), participants also needed to have quadriceps weakness, defined as a peak torque $\leq 80 \%$ of the predicted value (20). Individuals with known neuromuscular diseases; hip, leg and/or knee disorders including metal implants; cardiac pacemaker and/or internal cardiac defibrillator were ineligible. The Medical Ethical Committee of the Maastricht University Medical Centre+ (MEC 09-3-072) approved this study, which conformed to the principles outlined in the World Medical Association declaration of Helsinki which was revised in Seoul (21). Details of the trial were registered at http://www.trialregister.nl (NTR2322) before subject enrolment. All patients gave written informed consent to participate.

\section{AsSESSMENTS}

\section{Medical history}

During the medical history, the number of patient-reported COPD exacerbations in the 12 months prior to assessment, the usage of long-term oxygen therapy (LTOT) and all respiratory and non-respiratory medications prescribed by secondary care respiratory physicians were routinely recorded (22).

\section{Pulmonary function and arterial blood gases}

Post-bronchodilator forced expiratory volume in one second $\left(\mathrm{FEV}_{1}\right)$ and forced vital capacity (FVC) were determined using spirometry (MasterScreen ${ }^{\circledR}$ Body, Carefusion, Houten, the Netherlands). Diffusing capacity for carbon monoxide ( $\left.D L_{c o}\right)$ was determined using the single breath method. All values were related to reference values (23). Arterial oxygen tension $\left(\mathrm{PaO}_{2}\right)$, arterial carbon dioxide tension $\left(\mathrm{PaCO}_{2}\right)$ were measured with a blood gas analyzer (GEM4000, Instrumentation Laboratory, Peachtree City, USA).

\section{Exercise performance}

A supervised symptom-limited cardiopulmonary incremental cycle test on an electrically braked cycle ergometer (Carefusion, Houten, the Netherlands) was conducted, as described before (24). Peak aerobic capacity (only in subjects without oxygen supplement, $n=55$ ) and peak work rate were determined. A constant work-rate cycling endurance test was performed at $75 \%$ of the peak work rate (25). Functional exercise capacity was measured using the six-minute walk test, which was performed twice (26). The best 6-minute walk distance (6MWD) was used for further analyses.

\section{Quadriceps muscle function}

Quadriceps muscle function (peak strength and endurance) was measured using a Biodex (Biodex System 4 Pro, Biodex Medical Systems, Inc., New York, USA) (24). The reliability of this method in patients with COPD has been demonstrated previously (27). 
Peak muscle strength (Newtonmeter; Nm) and muscle endurance (Total work; TW) were measured isokinetically. The participants performed thirty volitional maximal contractions at an angular velocity of $90^{\circ}$ per second. The measurement was performed twice, and best values were used for further analyses.

\section{Problematic activities of daily life}

The COPM, a semi-structured interview, was used to assess problematic activities of daily life (ADLs) (28). Problematic ADLs were categorized into four domains: self-care, productivity, leisure and mobility (11).

\section{Dyspnea and health status}

The Medical Research Council (MRC) dyspnea scale was used to assess symptoms of dyspnea (19). Health status was measured with the St. George's Respiratory Questionnaire (SGRQ) (29). Scores can range from 0 (optimal) to 100 points (worst).

\section{Comorbidities}

The degree of self-reported comorbidities was measured using the Charlson comorbidity index (30). Moreover, the following comorbidities were objectified, as described before (7):

Body composition abnormalities

Body mass index (BMI, defined as body weight divided by squared height) and fat-free mass index (FFMI), defined as fat free mass divided by squared height) were determined, and classified as obesity $\left(\mathrm{BMI}>30 \mathrm{~kg} / \mathrm{m}^{2}\right)$, underweight $\left(\mathrm{BMl}<21 \mathrm{~kg} / \mathrm{m}^{2}\right)$, and/or muscle wasting (FFMI $<14.62 \mathrm{~kg} / \mathrm{m}^{2}$ in women and $\mathrm{FFMl}<17.05 \mathrm{~kg} / \mathrm{m}^{2}$ in men) (31). In addition, bone mineral density (BMD of the hip, lumbar spine and whole body region, expressed as T-scores) were determined using dual-energy $\mathrm{x}$-ray absorptiometry (32). If the lowest of the three T-scores was <-2.5, the subject was defined as osteoporotic (33).

\section{Symptoms of anxiety and depression}

Symptoms of anxiety and depression were measured using the Hospital Anxiety and Depression Scale (HADS) (34). Scores can range from 0 (optimal) to 21 points (worst). A score of 10 points or more was defined as increased symptoms of anxiety and/or depression (3,34).

Hyperglycemia, anemia, dyslipidemia and systemic inflammation Routinely, a post-absorptive venous blood sample was collected from the patients in the fasted state to analyze glucose, hemoglobin, triglycerides, high density lipoprotein (HDL) and creatinine. 
A fasting glucose level $>5.6 \mathrm{mmol} / \mathrm{l}$ was defined as hyperglycemia (35); anemia was defined as a hemoglobin level $<13 \mathrm{~g} / \mathrm{dl}(8.1 \mathrm{mmol} / \mathrm{l}$, men) or $<12 \mathrm{~g} / \mathrm{dl}(7.5 \mathrm{mmol} / \mathrm{l}$, women) (36); dyslipidemia was defined as a triglyceride level above $1.7 \mathrm{mmol} / \mathrm{I}$ or a HDL cholesterol level below $1.03 \mathrm{mmol} / \mathrm{I}$ (men) or below $1.29 \mathrm{mmol} / \mathrm{l}$ (women) (37).

\section{Renal impairment}

Renal function was established by the estimated glomerular filtration rate (eGFR), using the Cockroft-Gault formula (38). Chronic kidney disease was defined as eGFR<60 $\mathrm{ml} / \mathrm{min}$, corresponding with stage 3 chronic kidney disease according to the the National Kidney Foundation Kidney Disease Outcome Quality Initiative (NKF KDOQI) guidelines (39).

\section{Cardiovascular abnormalities}

Peripheral blood pressure was measured three times with interval of 5 minutes, after 15 minutes of supine rest in early morning time. Mean values were calculated. Hypertension grade 1 or higher was based on cut-off values of $>140 \mathrm{mmHg}$ for systolic blood pressure and $>90 \mathrm{mmHg}$ for diastolic blood pressure (40).

A resting ECG was obtained and the Cardiac Infarction Injury Score (CIIS) was scored by a cardiologist (NHMKU-L) blinded for medical history and outcome measures. CIIS is an ECG classification system that was developed as a diagnostic tool to determine the presence of myocardial infarctions. It is based on the power of certain electrocardiographic characteristics to discriminate between myocardial infarction patients and healthy individuals. These characteristics are weighted and combined into a single score (41). Myocardial infarction was defined as a CIIS $\geq 20$ (41).

\section{Statistical analyses}

All statistical analyses were performed using SPSS for Windows, Version 17.0.1 (SPSS, Inc., Chicago, II, USA) and GraphPad Prism Version 4.03 (GraphPad Software, Inc., La Jolla, USA). Data were presented as mean \pm standard deviation, as median and interquartile range, or as frequencies, as appropriate. Gender differences were assessed using the Student's t-test, $\chi^{2}$ test or Mann-Whitney $U$ test. We additionally used the coefficient of variation (the ratio of the standard deviation to the mean) to present the degree of variability. A priori, the level of significance was set at $\leq 0.05$. 


\section{RESULTS}

\section{Charateristics of Whole sample}

In total, 120 individuals with COPD fulfilled all inclusion criteria and consented to participate in the DICES trial. Nevertheless, three individuals with COPD GOLD B were excluded from the current analysis to focus specifically on individuals with GOLD D.

The remaining 117 individuals with GOLD D had severe to very severe COPD and an impaired diffusion capacity (Table 6.1). 52\% of the patients used long-term oxygen therapy and $75 \%$ patients reported 1 or more exacerbations in the previous 12 months (Figure 6.1). Various types of respiratory and non-respiratory medications were used (Supplemental Table S6.1). On average, participants had a decreased exercise capacity (Table 6.2), lower-limb muscle dysfunction (Table 6.3), and a decreased health status (SGRQ total score: $63.8 \pm 13.2$ points). Even though all participants reported an MRC dyspnea grade of $4 / 5$ and GOLD D, the clinical characteristics showed a broad range of values (Figures 6.2A to 6.2E). Indeed, peak work rate ranged from 4 to 68 watts; the 6min walk distance ranged from 22 to 544 meters; health status ranged from 22 to 88 points; anxiety scores ranged from 1 to 19 points; and depression scores ranged from 1 to 18 points. Moreover, the coefficient of variation ranged from 0.52 for bone mineral density to 0.49 for cycle endurance time and HADS anxiety score. Moreover, 42 problematic activities of daily were reported, with walking, showering, and household activities as the most prevalent (see Supplement Tables S6.2 and S6.3 for all details). Finally, $87 \%$ of the participants scored $\geq 1$ objectified comorbidities (Charlson comorbidity index score: $1.57 \pm 0.82$ points). Hyperglycaemia, low muscle mass, and symptoms of anxiety were most prevalent (Table 6.4; Figure 6.3).

Table 6.1 General characteristics.

\begin{tabular}{|c|c|c|c|c|c|}
\hline & & $\begin{array}{c}\text { Total sample } \\
\mathrm{n}=117\end{array}$ & $\begin{array}{l}\text { Men } \\
n=61\end{array}$ & $\begin{array}{c}\text { Women } \\
n=56\end{array}$ & P-value \\
\hline Age & years & $64.7 \pm 8.1(0.13)$ & $66.7 \pm 8.3$ & $62.6 \pm 7.5$ & 0.005 \\
\hline $\mathrm{FEV}_{1}$ & liters & $0.84 \pm 0.34(0.40)$ & $0.94 \pm 0.38$ & $0.72 \pm 0.26$ & $<0.000$ \\
\hline $\mathrm{FEV}_{1}$ & $\%$ predicted & $33 \pm 13(0.39)$ & $31 \pm 13$ & $34 \pm 13$ & 0.254 \\
\hline $\mathrm{FEV}_{1} / \mathrm{VC} \max$ & $\%$ & $31 \pm 11(0.35)$ & $30 \pm 12$ & $33 \pm 9$ & 0.139 \\
\hline $\mathrm{DL}_{\mathrm{CO}}$ & $\%$ predicted & $41 \pm 15(0.37)$ & $41 \pm 16$ & $41 \pm 14$ & 0.997 \\
\hline RV & $\%$ & $201 \pm 53(0.26)$ & $198 \pm 58$ & $204 \pm 47$ & 0.537 \\
\hline $\mathrm{PaO}_{2}$ & $\mathrm{kPa}$ & $9.7 \pm 1.6(0.16)$ & $9.9 \pm 1.7$ & $9.4 \pm 1.5$ & 0.079 \\
\hline $\mathrm{PaCO}_{2}$ & $\mathrm{kPa}$ & $5.7 \pm 1.2(0.21)$ & $5.5 \pm 1.2$ & $5.9 \pm 1.2$ & 0.108 \\
\hline $\mathrm{SaO}_{2}$ & $\%$ & $95 \pm 2(0.02)$ & $96 \pm 2$ & $95 \pm 3$ & 0.111 \\
\hline LTOT & $\%$ & 52 & 48 & 57 & 0.301 \\
\hline
\end{tabular}

Values expressed as mean \pm SD (coefficient of variation) or percentages. GOLD=Global Initiative on Obstructive Lung Diseases; LTOT=long-term oxygen therapy; $\mathrm{FEV}_{1}=$ forced expiratory volume in one second; VC max=maximum vital capacity; $\mathrm{DL}_{\mathrm{co}}=$ diffusion capacity of the lung for carbon monoxide; RV=residual volume; $\mathrm{PaO}_{2}=$ resting arterial oxygen tension; $\mathrm{PaCO}_{2}=$ resting arterial carbon dioxide tension; $\mathrm{SaO}_{2}=$ resting arterial oxygen tension; $\mathrm{kPa}=$ kilopascal. 
Figure 6.1 Number of exacerbations in the past 12 months.

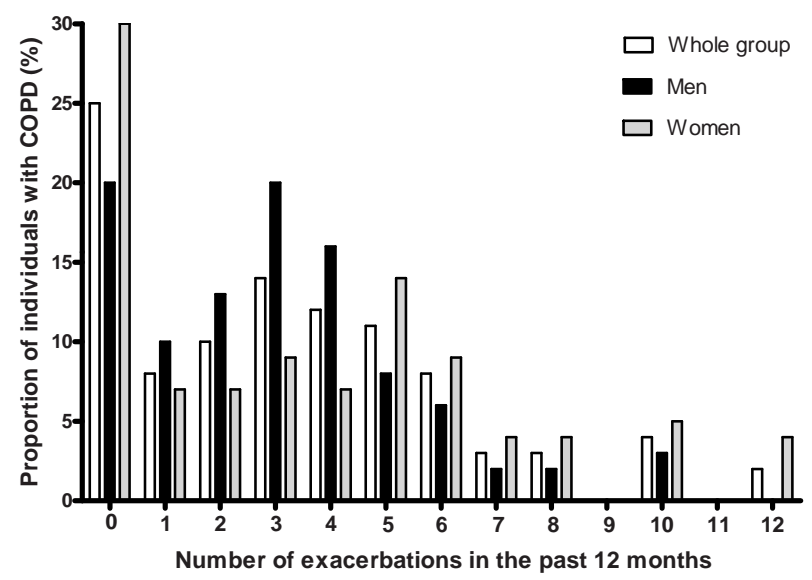

Table 6.2 Exercise performance.

\begin{tabular}{llcccc}
\hline & & Total & Men & Women & P-value \\
\hline Cardiopulmonary exercise test & watts & $\mathrm{n}=101$ & $\mathrm{n}=53$ & $\mathrm{n}=48$ & \\
Peak load & Watts/kg & $0.96 \pm 0.28(0.29)$ & $0.90 \pm 0.21$ & $1.03 \pm 0.33$ & 0.024 \\
Peak load/FFM & $\mathrm{ml} / \mathrm{min}$ & $820 \pm 159(0.19)$ & $834 \pm 153$ & $802 \pm 168$ & 0.467 \\
Peak VO & $\mathrm{ml} / \mathrm{min} / \mathrm{kg}$ & $17.8+4.0(0.22)$ & $16.0 \pm 2.8$ & $20.3 \pm 4.0$ & $<0.001$ \\
Peak VO & /FFM & $\mathrm{n}=93$ & $\mathrm{n}=51$ & $\mathrm{n}=42$ & \\
Constant work rate test & & $192 \pm 95(0.49)$ & $199 \pm 108$ & $184 \pm 76$ & 0.468 \\
Cycle time & seconds & $\mathrm{n}=117$ & $\mathrm{n}=61$ & $\mathrm{n}=56$ & \\
6 minute walk test & & $322 \pm 92(0.29)$ & $314 \pm 87$ & $331 \pm 97$ & 0.321 \\
6MWD & meters & 56 & 62 & 48 & 0.127 \\
6MWD $<350$ meters & $\%$ & $52 \pm 16(0.31)$ & $47 \pm 14$ & $57 \pm 17$ & 0.002 \\
6MWD & $\%$ predicted & & & & \\
\hline
\end{tabular}

Values expressed as mean $\pm \mathrm{SD}$ (coefficient of variation) or percentages. Peak $\mathrm{VO}_{2}=$ peak oxygen uptake in $\mathrm{ml} / \mathrm{min}$.; peak $\mathrm{VE}=$ peak minute ventilation in liters; peak $\mathrm{HR}=$ peak heart rate; bpm=beats per minute; \% $\mathrm{MVV}=$ percentage maximal voluntary ventilation; $\mathrm{tSaO}_{2}=$ transcutaneous oxygen saturation; $6 \mathrm{MWD}=6$-minute walk distance; $\mathrm{kg}=$ kilogram; $\mathrm{min}=$ minute; $\mathrm{ml}=$ milliliter.

\section{Men Versus WOMEN}

The degree of airflow limitation (\% predicted), $\mathrm{DL}_{\mathrm{CO}}$ and arterial blood gases were similar between men and women (Table 6.1). Peak work load and peak $\mathrm{VO}_{2}$ (after correction for lower-limb muscle mass) and 6MWD expressed ( $\%$ predicted) were significantly higher in women compared to men (all $p<0.05$; Table 6.2). The performance and satisfaction scores for self-reported problematic ADLs on the COPM were similar between men and women ( $3.5 \pm 1.7$ versus $3.5 \pm 1.9$ points; and $3.0 \pm 1.8$ versus $3.1 \pm 2.1$ points, respectively). Women reported walking, household activities and showering to be to the most important problematic ADLs, while men reported walking, showering and stair climbing. Disease-specific health status (SGRQ total score: 
$65.0 \pm 12.1$ versus $62.6 \pm 14.3$ points, respectively), BMI and bone mineral density were not different between men and women (Table 6.3). Women had significantly higher symptom scores of anxiety $(p=0.024)$, and a higher proportion of obesity, dyslipidemia and renal impairment (Figure 6.3).

Figure 6.1 Heterogeneity in clinical characteristics.
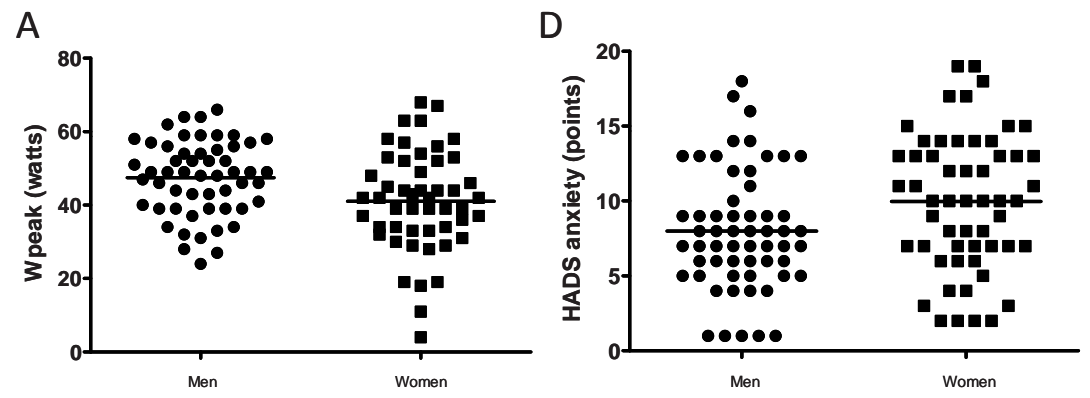

B

$E$
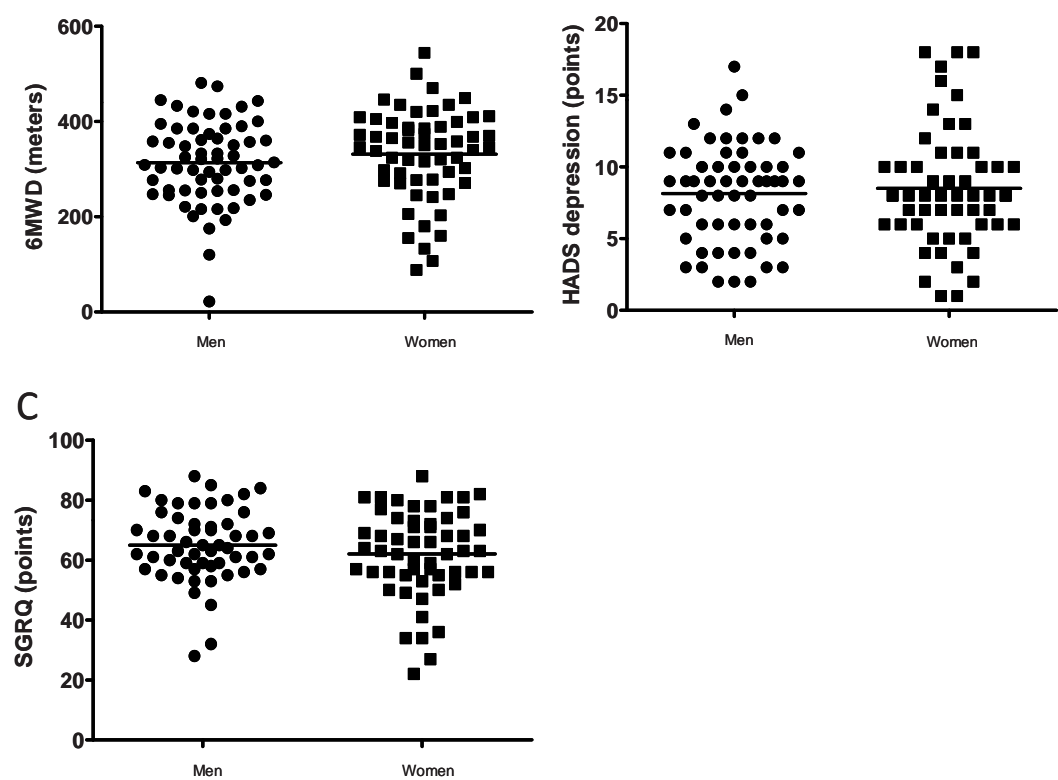

The distribution is shown for men (circles) and women (squares). The median is shown with a black line. A: Wpeak (peak work rate); B: 6MWD (6-minute walk test); C: SGRQ (health status); D: HADS anxiety; E: HADS depression. 
Table 6.3 Body composition and muscle function.

\begin{tabular}{lccccc}
\hline & & Total & Men & Women & P-value \\
\hline Body composition & & $\mathrm{n}=117$ & $\mathrm{n}=61$ & $\mathrm{n}=56$ & \\
$\mathrm{BMI}$ & $\mathrm{kg} / \mathrm{m}^{2}$ & $24.7 \pm 5.0(0.20)$ & $24.1 \pm 4.7$ & $25.3 \pm 5.2$ & 0.217 \\
FFMI & $\mathrm{kg} / \mathrm{m}^{2}$ & $16.5 \pm 2.0(0.12)$ & $17.4 \pm 1.9$ & $15.5 \pm 1.6$ & $<0.001$ \\
Bone mineral density & $\mathrm{T}-\mathrm{score}$ & $-2.1 \pm 1.1(0.52)$ & $-2.0 \pm 1.3$ & $-2.1 \pm 0.9$ & 0.656 \\
Isokinetic quadriceps muscle function & $\mathrm{n}=117$ & $\mathrm{n}=61$ & $\mathrm{n}=56$ & \\
Peak torque & $\mathrm{Nm}$ & $76.0 \pm 26.4(0.35)$ & $86.6 \pm 27.7$ & $64.5 \pm 19.3$ & $<0.001$ \\
Peak torque & $\%$ predicted & $54 \pm 16(0.30)$ & $51 \pm 16$ & $57 \pm 16$ & 0.075 \\
Peak torque/FFM & Nm/kg & $1.6 \pm 0.5(0.31)$ & $1.6 \pm 0.5$ & $1.6 \pm 0.5$ & 0.810 \\
Total work & Joules & $1175 \pm 480(0.41)$ & $1308 \pm 542$ & $1029 \pm 353$ & 0.001 \\
Total work/FFM & Joules/kg & $25.2 \pm 8.9(0.35)$ & $24.7 \pm 9.4$ & $25.9 \pm 8.3$ & 0.468 \\
\hline
\end{tabular}

Values expressed as mean \pm SD (coefficient of variation). $B M I=$ body mass index; FFM=fat-free mass; FFMI=fatfree mass index; $\mathrm{kg}=$ kilogram; $\mathrm{m}^{2}=$ squared meter; $\mathrm{Nm}=$ newtonmeter.

Figure 6.3 Prevalence of objectified comorbidities.

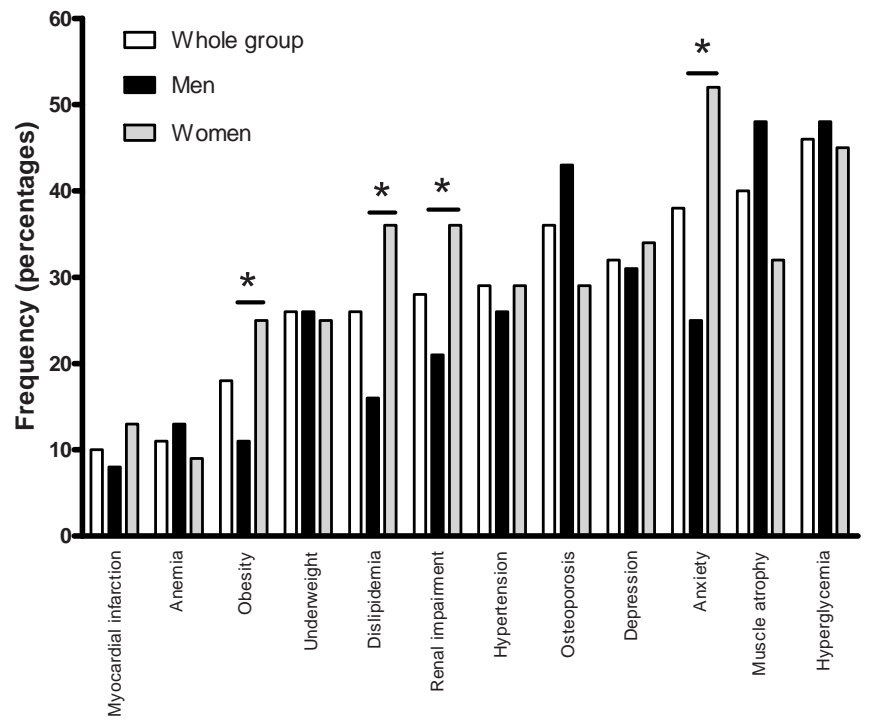


Table 6.4 Mood and health status.

\begin{tabular}{llcccc}
\hline & & Total & Men & Women & P-value \\
\hline HADS & & $\mathrm{n}=110$ & $\mathrm{n}=57$ & $\mathrm{n}=53$ & \\
Anxiety & points & $8.9 \pm 4.4(0.49)$ & $8.0 \pm 4.0$ & $9.9 \pm 4.6$ & 0.024 \\
Anxiety $\geq 10$ points & $\%$ & 38 & 25 & 52 & 0.003 \\
Depression & points & $8.4 \pm 3.8(0.45)$ & $8.1 \pm 3.5$ & $8.6 \pm 4.2$ & 0.497 \\
Depression $\geq 10$ points & $\%$ & 32 & 31 & 34 & 0.749 \\
SGRQ & & $\mathrm{n}=109$ & $\mathrm{n}=55$ & $\mathrm{n}=54$ & \\
Symptoms & points & $66.3 \pm 16.9(0.25)$ & $66.5 \pm 16.3$ & $66.2 \pm 17.7$ & 0.939 \\
Activity & points & $81.7 \pm 16.4(0.20)$ & $83.3 \pm 16.4$ & $80.1 \pm 16.4$ & 0.321 \\
Impact & points & $53.1 \pm 17.5(0.33)$ & $54.3 \pm 15.8$ & $51.9 \pm 19.2$ & 0.477 \\
Total score & points & $63.9 \pm 13.2(0.21)$ & $65.0 \pm 12.0$ & $62.8 \pm 14.3$ & 0.388 \\
\hline
\end{tabular}

Values expressed as mean \pm SD (coefficient of variation) or percentages. HADS=Hospital Anxiety and Depression Scale; SGRQ=St. George's Respiratory Questionnaire.

\section{DISCUSSION}

The current findings emphasize that COPD is a heterogeneous disease whose clinical presentation varies significantly, even in individuals with very severe COPD with the same degree of dyspnea and all classified as GOLD D. Moreover, significant gender differences were found for exercise performance, lower-limb muscle function and various comorbidities.

Previously, heterogeneity in clinical outcomes has been found after stratification for GOLD stage II to IV, suggesting that the degree of airflow limitation does not capture the clinical heterogeneity of $\operatorname{COPD}(5,42)$. The current study shows a broad heterogeneity in exercise capacity, lower-limb muscle function, mood status, health status, exacerbations and comorbidities in individuals with COPD GOLD D and MRC dyspnea grade $4 / 5$. The coefficient of variation for various clinical characteristics (i.e. $\mathrm{FEV}_{1}$ : 0.33 versus 0.39 ; $6 \mathrm{MWD}$ : 0.33 versus 0.31 ; and $\mathrm{BMI}: 0.22$ versus 0.20 (5)) seems comparable between the 2007 GOLD classification (based solely on degree of airflow limitation) (8) and the 2013 GOLD classification (43). This suggests that the MRC dyspnea scale as well as the new GOLD classification may provide clinicians with a global impression of the clinical status of patients with COPD. However, patients with COPD need to be assessed individually to be able to provide a patient-tailored comprehensive COPD management program. Therefore, it seems reasonable to state that the new GOLD classification (9) cannot be the basis for personalized COPD management. The variability in clinical characteristics and comorbidities in this group of individuals with COPD GOLD D may partially explain the odds of survival in this subgroup $(44,45)$. Obviously, this needs to be corroborated prospectively. 
Multi-morbidity occurs frequently in the general population (46). Comorbidities occur also frequently in patients with COPD entering pulmonary rehabilitation $(7,47)$. Comparable frequencies of comorbidities have been found in the current study (Figure 6.3). This emphasizes again the complexity of patients with COPD entering pulmonary rehabilitation. For example, patients with COPD with increased symptoms of anxiety and/or depression have worse prognosis $(48,49)$. Moreover, individuals with COPD who also had osteoporosis at baseline had worst response on the 6-minute walk test following pulmonary rehabilitation (50).

Even though the degree of dyspnea, the degree of airflow limitation and the new GOLD group were similar between women and men in the present study, still significant gender differences were found. The female patients had a better exercise performance compared to men, after adjustment for FFM (Table 6.2). Even though the women were significantly younger (mean difference: 3 years), this does not seem to explain the mean difference in peak aerobic capacity $(4.3 \mathrm{ml} / \mathrm{min} / \mathrm{kg} \mathrm{FFM} ; 27 \%)$. Whether and to what extent this difference is due to changes in muscle structure and function, neurological function (51), cardiovascular function (52), hormonal exposures, health behaviors (53) or a combination thereof remains to be determined.

Women had a higher prevalence of obesity, anxiety, dyslipidemia and renal impairment. This suggests that gender-specific patterns of comorbidities may exist in COPD. Whether and to what extent gender-differences in comorbidities may contribute to the overall severity of COPD patients remains to be determined. At first sight, health status does not seem to be influenced by these differences as mean SGRQ scores were similar between women and men in the present study.

Obviously, the inclusion criteria of the DICES trial limits the external validity of the present findings. Nevertheless, previous studies have already shown a large heterogeneity in clinical outcomes, like 6MWD, after stratification for MRC dyspnea grade or GOLD stage $(5,12)$. Therefore, the authors believe that the heterogeneous clinical presentation of COPD is not limited to individuals with MRC 4/5 and COPD GOLD D.

To conclude, COPD patients with severe dyspnea and classified as high risk with more symptoms according to the new GOLD classification have a highly variable clinical presentation. Moreover, this heterogeneity remains after stratification for sex. Finally, clinically relevant gender-differences exist, although the degree of dyspnea and the degree of airflow limitation were comparable between women and men. These findings emphasize that patients with COPD entering a pulmonary rehabilitation program need to be assessed interdisciplinary and individually to be able to provide a patient-tailored program. Moreover, the new GOLD classification seems inappropriate to capture the clinical heterogeneity of patients with COPD. 


\section{REFERENCES}

1. Lopez AD, Mathers CD, Ezzati M, Jamison DT, Murray CJ. Global and regional burden of disease and risk factors, 2001: systematic analysis of population health data. Lancet. 2006;367(9524):1747-57.

2. Han MK, Agusti A, Calverley PM, Celli BR, Criner G, Curtis JL, et al. Chronic obstructive pulmonary disease phenotypes: the future of COPD. Am J Respir Crit Care Med. 2010;182(5):598-604.

3. Janssen DJ, Spruit MA, Uszko-Lencer NH, Schols JM, Wouters EF. Symptoms, comorbidities, and health care in advanced chronic obstructive pulmonary disease or chronic heart failure. Journal of palliative medicine. 2011;14(6):735-43.

4. Rabinovich RA, MacNee W. Chronic obstructive pulmonary disease and its comorbidities. Br J Hosp Med (Lond). 2011;72(3):137-45.

5. Agusti A, Calverley PM, Celli B, Coxson HO, Edwards LD, Lomas DA, et al. Characterisation of COPD heterogeneity in the ECLIPSE cohort. Respiratory research. 2010;11:122.

6. Spruit MA, Watkins ML, Edwards LD, Vestbo J, Calverley PM, Pinto-Plata V, et al. Determinants of poor 6-min walking distance in patients with COPD: the ECLIPSE cohort. Respir Med. 2010;104(6):849-57.

7. Vanfleteren LE, Spruit MA, Groenen M, Gaffron S, van Empel VP, Bruijnzeel PL, et al. Clusters of comorbidities based on validated objective measurements and systemic inflammation in patients with chronic obstructive pulmonary disease. Am J Respir Crit Care Med. 2013;187(7):728-35.

8. Rabe KF, Hurd S, Anzueto A, Barnes PJ, Buist SA, Calverley P, et al. Global strategy for the diagnosis, management, and prevention of chronic obstructive pulmonary disease: GOLD executive summary. Am J Respir Crit Care Med. 2007;176(6):532-55.

9. Vestbo J, Hurd SS, Agusti AG, Jones PW, Vogelmeier C, Anzueto A, et al. Global Strategy for the Diagnosis, Management and Prevention of Chronic Obstructive Pulmonary Disease, GOLD Executive Summary. Am J Respir Crit Care Med. 2012.

10. Bestall JC, Paul EA, Garrod R, Garnham R, Jones PW, Wedzicha JA. Usefulness of the Medical Research Council (MRC) dyspnoea scale as a measure of disability in patients with chronic obstructive pulmonary disease. Thorax. 1999;54(7):581-6.

11. Annegarn J, Meijer K, Passos VL, Stute K, Wiechert J, Savelberg HH, et al. Problematic activities of daily life are weakly associated with clinical characteristics in COPD. J Am Med Dir Assoc. 2012;13(3):284-90.

12. Spruit MA, Pennings HJ, Janssen PP, Does JD, Scroyen S, Akkermans MA, et al. Extra-pulmonary features in COPD patients entering rehabilitation after stratification for MRC dyspnea grade. Respir Med. 2007;101(12):2454-63.

13. Vaes AW, Wouters EF, Franssen FM, Uszko-Lencer NH, Stakenborg KH, Westra M, et al. Task-related oxygen uptake during domestic activities of daily life in patients with COPD and healthy elderly subjects. Chest. 2011;140(4):970-9.

14. Vanfleteren LE, Franssen FM, Uszko-Lencer NH, Spruit MA, Celis M, Gorgels AP, et al. Frequency and relevance of ischemic electrocardiographic findings in patients with chronic obstructive pulmonary disease. The American journal of cardiology. 2011;108(11):1669-74.

15. Lopez Varela MV, Montes de Oca M, Halbert RJ, Muino A, Perez-Padilla R, Talamo C, et al. Sex-related differences in COPD in five Latin American cities: the PLATINO study. Eur Respir J. 2010;36(5):1034-41.

16. de Torres JP, Casanova C, Hernandez C, Abreu J, Aguirre-Jaime A, Celli BR. Gender and COPD in patients attending a pulmonary clinic. Chest. 2005;128(4):2012-6.

17. Celli B, Vestbo J, Jenkins CR, Jones PW, Ferguson GT, Calverley PM, et al. Gender Differences in Mortality and Clinical Expressions of Patients with COPD: The TORCH Experience. Am J Respir Crit Care Med. 2010.

18. Spruit MA, Vanderhoven-Augustin I, Janssen PP, Wouters EF. Integration of pulmonary rehabilitation in COPD. Lancet. 2008;371(9606):12-3.

19. Mahler DA, Wells CK. Evaluation of clinical methods for rating dyspnea. Chest. 1988;93(3):580-6.

20. Borges $\mathrm{O}$. Isometric and isokinetic knee extension and flexion torque in men and women aged 20-70. Scand J Rehabil Med. 1989;21(1):45-53.

21. Wegmann H. The "new" Declaration of Helsinki. Jouirnal of International Biotechnology Law. 2009;6(4):173-6. 
22. Franssen FM, Spruit MA, Wouters EF. Determinants of polypharmacy and compliance with GOLD guidelines in patients with chronic obstructive pulmonary disease. Int J Chron Obstruct Pulmon Dis. 2011;6:493-501.

23. Quanjer PH, Tammeling GJ, Cotes JE, Pedersen OF, Peslin R, Yernault JC. Lung volumes and forced ventilatory flows. Report Working Party Standardization of Lung Function Tests, European Community for Steel and Coal. Official Statement of the European Respiratory Society. Eur Respir J Suppl. 1993;16:5-40.

24. Franssen FM, Broekhuizen R, Janssen PP, Wouters EF, Schols AM. Limb muscle dysfunction in COPD: effects of muscle wasting and exercise training. Med Sci Sports Exerc. 2005;37(1):2-9.

25. Hul van 't A, Gosselink R, Kwakkel G. Constant-load cycle endurance performance: test-retest reliability and validity in patients with COPD. J Cardiopulm Rehabil. 2003;23(2):143-50.

26. Hernandes NA, Wouters EF, Meijer K, Annegarn J, Pitta F, Spruit MA. Reproducibility of 6-minute walking test in patients with COPD. Eur Respir J. 2011;38(2):261-7.

27. Mathur S, Makrides L, Hernandez P. Test-retest reliability of isometric and isokinetic torque in patients with chronic obstructive pulmonary disease. Physiother Can. 2004;56:94-101.

28. Law M, Baptiste S, McColl M, Opzoomer A, Polatajko H, Pollock N. The Canadian occupational performance measure: an outcome measure for occupational therapy. Can J Occup Ther. 1990; 57(2):82-7.

29. Jones PW, Quirk FH, Baveystock CM. The St George's Respiratory Questionnaire. Respir Med. 1991;85 Suppl B:25-31; discussion 3-7.

30. Charlson ME, Pompei P, Ales KL, MacKenzie CR. A new method of classifying prognostic comorbidity in longitudinal studies: development and validation. J Chronic Dis. 1987;40(5):373-83.

31. Vestbo J, Prescott E, Almdal T, Dahl M, Nordestgaard BG, Andersen T, et al. Body mass, fat-free body mass, and prognosis in patients with chronic obstructive pulmonary disease from a random population sample: findings from the Copenhagen City Heart Study. Am J Respir Crit Care Med. 2006;173(1):79-83.

32. Graat-Verboom L, Smeenk FW, van den Borne BE, Spruit MA, Jansen FH, van Enschot JW, et al. Progression of osteoporosis in patients with COPD: a 3-year follow up study. Respir Med. 2012; 106(6):861-70.

33. Graat-Verboom L, Wouters EF, Smeenk FW, van den Borne BE, Lunde R, Spruit MA. Current status of research on osteoporosis in COPD: a systematic review. Eur Respir J. 2009;34(1):209-18.

34. Zigmond AS, Snaith RP. The hospital anxiety and depression scale. Acta Psychiatr Scand. 1983; 67(6):361-70.

35. Diagnosis and classification of diabetes mellitus. Diabetes care. 2010;33 Suppl 1:S62-9.

36. Nutritional anaemias. Report of a WHO scientific group. World Health Organization technical report series. 1968;405:5-37.

37. Alberti KG, Zimmet P, Shaw J. The metabolic syndrome--a new worldwide definition. Lancet. 2005; 366(9491):1059-62.

38. Cockcroft DW, Gault MH. Prediction of creatinine clearance from serum creatinine. Nephron. 1976; 16(1):31-41.

39. KDOQI Clinical Practice Guidelines and Clinical Practice Recommendations for Diabetes and Chronic Kidney Disease. American journal of kidney diseases : the official journal of the National Kidney Foundation. 2007;49(2 Suppl 2):S12-154.

40. 1999 World Health Organization-International Society of Hypertension Guidelines for the Management of Hypertension. Guidelines Subcommittee. Journal of hypertension. 1999;17(2):151-83.

41. Rautaharju PM, Warren JW, Jain U, Wolf HK, Nielsen CL. Cardiac infarction injury score: an electrocardiographic coding scheme for ischemic heart disease. Circulation. 1981;64(2):249-56.

42. Huijsmans RJ, de Haan A, ten Hacken NN, Straver RV, van't Hul AJ. The clinical utility of the GOLD classification of COPD disease severity in pulmonary rehabilitation. Respir Med. 2008;102(1):162-71.

43. Vestbo J, Hurd SS, Agusti AG, Jones PW, Vogelmeier C, Anzueto A, et al. Global strategy for the diagnosis, management, and prevention of chronic obstructive pulmonary disease: GOLD executive summary. Am J Respir Crit Care Med. 2013;187(4):347-65.

44. Lange P, Marott JL, Vestbo J, Olsen KR, Ingebrigtsen TS, Dahl M, et al. Prediction of the clinical course of chronic obstructive pulmonary disease, using the new GOLD classification: a study of the general population. Am J Respir Crit Care Med. 2012;186(10):975-81. 
45. Spruit MA, Polkey MI, Celli B, Edwards LD, Watkins ML, Pinto-Plata V, et al. Predicting outcomes from 6minute walk distance in chronic obstructive pulmonary disease. J Am Med Dir Assoc. 2012;13(3):291-7.

46. Barnett K, Mercer SW, Norbury M, Watt G, Wyke S, Guthrie B. Epidemiology of multimorbidity and implications for health care, research, and medical education: a cross-sectional study. Lancet. 2012;380(9836):37-43.

47. Janssen DJ, Spruit MA, Leue C, Gijsen C, Hameleers H, Schols JM, et al. Symptoms of anxiety and depression in COPD patients entering pulmonary rehabilitation. Chron Respir Dis. 2010;7(3):147-57.

48. Laurin C, Moullec G, Bacon SL, Lavoie KL. Impact of anxiety and depression on chronic obstructive pulmonary disease exacerbation risk. Am J Respir Crit Care Med. 2012;185(9):918-23.

49. de Voogd JN, Wempe JB, Koeter GH, Postema K, van Sonderen E, Ranchor AV, et al. Depressive symptoms as predictors of mortality in patients with COPD. Chest. 2009;135(3):619-25.

50. Crisafulli E, Gorgone P, Vagaggini B, Pagani M, Rossi G, Costa F, et al. Efficacy of standard rehabilitation in COPD outpatients with comorbidities. Eur Respir J. 2010;36(5):1042-8.

51. Clark BC, Manini TM. Sarcopenia =/=dynapenia. J Gerontol A Biol Sci Med Sci. 2008;63(8):829-34.

52. Cunningham DA, Rechnitzer PA, Pearce ME, Donner AP. Determinants of self-selected walking pace across ages 19 to 66. Journal of gerontology. 1982;37(5):560-4.

53. Hughes VA, Frontera WR, Wood M, Evans WJ, Dallal GE, Roubenoff R, et al. Longitudinal muscle strength changes in older adults: influence of muscle mass, physical activity, and health. J Gerontol A Biol Sci Med Sci. 2001;56(5):B209-17. 


\section{SUPPLEMENTAL TABLES}

Table S6.1 Numbers of patients using various categories of medications.

\begin{tabular}{|c|c|c|}
\hline \multicolumn{2}{|c|}{ Medication } & \multirow{2}{*}{$\frac{\mathbf{N}}{62}$} \\
\hline 1 & SABA Short acting $\beta 2$-agonists & \\
\hline 2 & SAMA Short-acting anticholinergics (SAAC) & 13 \\
\hline 3 & SABA + SAMA Short-acting combinations (COMBI) & 47 \\
\hline 4 & LABA Long-acting $\beta 2$-agonists & 26 \\
\hline 5 & LAMA Long-acting anticholinergics & 95 \\
\hline 6 & ICS Inhaled corticosteroids alone & 26 \\
\hline 7 & ICS + LABA Inhaled corticosteroids in combination with LABA & 86 \\
\hline 8 & THEOLAIR & 21 \\
\hline 9 & ORAL CORTICOSTEROIDS & 58 \\
\hline 10 & ANTI-LEUKOTRIENES & 3 \\
\hline 11 & ANTIHISTAMINICUM & 8 \\
\hline 12 & NASAL CORTICOSTEROIDS & 1 \\
\hline 13 & ACE OR ARB & 32 \\
\hline 14 & BETA BLOCKERS & 17 \\
\hline 15 & CALCIUM BLOCKERS & 24 \\
\hline 16 & ANTI ARRYTHMICA & 8 \\
\hline 17 & NITRATES & 15 \\
\hline 18 & DIURETICS & 41 \\
\hline 19 & ANTILIPAEMICA & 39 \\
\hline 20 & ANTIAGGREGATES & 36 \\
\hline 21 & COUMARINES & 14 \\
\hline 22 & ORAL ANTIDIABETICA / INSULIN & 11 \\
\hline 23 & CALCIUM SUPPLETION and/or VITAMIN D & 34 \\
\hline 24 & BISFOSFONATES & 39 \\
\hline 25 & ANTIDEPRESSIVES & 24 \\
\hline 26 & ANXIOLYTICS and SLEEP MEDICATION & 39 \\
\hline $27 a$ & PARACETAMOL & 14 \\
\hline $27 b$ & NSAIDs & 12 \\
\hline $27 c$ & MORPHINE & 7 \\
\hline $27 d$ & CODEINE & 10 \\
\hline $27 e$ & OTHER PAINKILLERS & 0 \\
\hline 28 & PPI/ANTACIDA & 73 \\
\hline 29 & ANTIBIOTICS & 21 \\
\hline 30 & ACETYLCYSTEIN & 32 \\
\hline 31 & OTHER MEDICATION & 52 \\
\hline 32 & H1N1 vaccination - influvac - pneumovax & 0 \\
\hline
\end{tabular}


Table S6.2 Canadian Occupational Performance Measure.

\begin{tabular}{|c|c|c|c|c|c|}
\hline & & $\begin{array}{c}\text { Total } \\
\mathrm{n}=117\end{array}$ & $\begin{array}{c}\text { Males } \\
n=61\end{array}$ & $\begin{array}{c}\text { Females } \\
n=56\end{array}$ & P-value \\
\hline \multicolumn{6}{|l|}{ Domain } \\
\hline \multirow[t]{2}{*}{ Self-care } & $\mathrm{P}$ & $4.2 \pm 1.7(0.40)$ & $4.2 \pm 1.6$ & $4.1 \pm 1.8$ & 0.881 \\
\hline & $\mathrm{S}$ & $3.5 \pm 2.0(0.57)$ & $3.5 \pm 1.8$ & $3.6 \pm 2.3$ & 0.762 \\
\hline \multirow[t]{2}{*}{ Mobility } & $\mathrm{P}$ & $3.2 \pm 1.7(0.05)$ & $3.4 \pm 1.6$ & $3.2 \pm 1.9$ & 0.348 \\
\hline & $\mathrm{S}$ & $2.8 \pm 1.8(0.64)$ & $2.8 \pm 1.6$ & $2.8 \pm 2.1$ & 0.823 \\
\hline \multirow[t]{2}{*}{ Productivity } & $\mathrm{P}$ & $3.2 \pm 1.8(0.56)$ & $3.3 \pm 1.7$ & $3.2 \pm 1.8$ & 0.939 \\
\hline & $\mathrm{S}$ & $2.9 \pm 2.1(0.72)$ & $2.7 \pm 1.9$ & $3.0 \pm 2.2$ & 0.450 \\
\hline \multirow[t]{2}{*}{ Leisure } & $\mathrm{P}$ & $3.5 \pm 2.3(0.35)$ & $3.3 \pm 2.1$ & $3.7 \pm 2.6$ & 0.473 \\
\hline & $\mathrm{S}$ & $3.0 \pm 2.1(0.70)$ & $2.8 \pm 2.0$ & $3.3 \pm 2.3$ & 0.397 \\
\hline
\end{tabular}

Values expressed as mean \pm SD (coefficient of variation). $\mathrm{P}=$ performance (points); $\mathrm{S}=$ satisfaction (points).

Table S6.3 TOP 10 problematic ADLs.

\begin{tabular}{|c|c|c|c|}
\hline & Total & Males & Females \\
\hline & $n=117$ & $n=61$ & $n=56$ \\
\hline \multirow[t]{2}{*}{ Rank } & Activity & Activity & Activity \\
\hline & $\%$ & $\%$ & $\%$ \\
\hline \multirow[t]{2}{*}{1.} & Walking & Walking & Walking \\
\hline & 90.8 & 95.2 & 86.2 \\
\hline \multirow[t]{2}{*}{2.} & Showering & Showering & Household activities* \\
\hline & 50.0 & 53.2 & 70.7 \\
\hline \multirow[t]{2}{*}{3.} & Household activities* & Stair climbing & Showering \\
\hline & 49.1 & 40.3 & 46.6 \\
\hline \multirow[t]{2}{*}{4.} & Stair climbing & Dress/undress & Stair climbing \\
\hline & 39.2 & 37.1 & 37.9 \\
\hline \multirow[t]{2}{*}{5.} & Dress/undress & Household activities* & Go shopping \\
\hline & 32.5 & 29.0 & 29.3 \\
\hline \multirow[t]{2}{*}{6.} & Go shopping & Gardening & Dress/undress \\
\hline & 20.8 & 16.1 & 27.6 \\
\hline \multirow[t]{2}{*}{7.} & Social activities & Go shopping & Social activities \\
\hline & 16.7 & Transfers $^{\ddagger}$ & 25.9 \\
\hline \multirow[t]{2}{*}{8.} & Selfcare & Cycling & Selfcare \\
\hline & 15.8 & 12.9 & 20.7 \\
\hline \multirow[t]{2}{*}{9.} & Preparing food & & Preparing food \\
\hline & 13.3 & & 17.2 \\
\hline \multirow[t]{3}{*}{10.} & Cycling & Preparing food & Cycling \\
\hline & Gardening & 9.7 & Activities with grandchildren \\
\hline & 12.5 & & 12.1 \\
\hline
\end{tabular}

* Household activities such as making the bed, vacuuming, cleaning the bathroom etc.; ${ }^{\ddagger}$ Transfers such as to step in and out of the car, bed and/or/bath. 



\section{CHAPTER 7}

Efficacy of lower-limb muscle training modalities in

severely dyspneic individuals with COPD and

quadriceps muscle weakness: results from

the DICES trial

Maurice JH Sillen, Frits ME Franssen, Jeannet ML Delbressine, Anouk W Vaes,

Emiel FM Wouters, Martijn A Spruit

Thorax, in press 


\section{ABSTRACT}

\section{Rationale}

Strength training and neuromuscular electrical stimulation (NMES) improve lower-limb muscle function in dyspneic individuals with chronic obstructive pulmonary disease (COPD). However, high-frequency NMES (HF-NMES) and strength training have never been compared head-to-head; and effects of low-frequency NMES (LF-NMES) have never been studied in COPD. Therefore, the optimal training modality to improve lower-limb muscle function, exercise performance and other patient-related outcomes in individuals with severe COPD remains unknown.

\section{Objectives}

The aim was to study prospectively the efficacy of HF-NMES $(75 \mathrm{~Hz})$, LF-NMES $(15 \mathrm{~Hz})$ or strength training in severely dyspneic individuals with COPD with quadriceps muscle weakness at baseline.

\section{Methods}

120 individuals with COPD (FEV 1 : $33 \pm 1 \%$ predicted, men: $52 \%$, age: $64.8 \pm 0.8$ years) were randomized to HF-NMES, LF-NMES or strength training as part of a comprehensive inpatient pulmonary rehabilitation program. No treadmill walking or stationary cycling was provided.

\section{Measurements and main results}

Groups were comparable at baseline. Quadriceps muscle strength increased following HF-NMES (+10.8 Newton-meter, $(\mathrm{Nm})$ ) or strength training (+6.1 Nm; both $\mathrm{p}<0.01)$, but not following LF-NMES $(+1.4 \mathrm{Nm} ; \mathrm{p}=0.43)$. Quadriceps muscle endurance, exercise performance, exercise-induced symptoms of dyspnea and fatigue improved significantly compared with baseline following HF-NMES, LF-NMES or strength training. The increase in quadriceps muscle strength and muscle endurance was greater after HF-NMES than after LF-NMES.

\section{Conclusions}

HF-NMES is equally effective as strength training in severely dyspneic individuals with COPD and muscle weakness in strengthening the quadriceps muscles and thus may be a good alternative in this particular group of patients. HF-NMES, LF-NMES and strength training were effective in improving exercise performance in severely dyspneic individuals with COPD and quadriceps weakness. 


\section{INTRODUCTION}

Lower-limb muscle dysfunction is a prominent extra-pulmonary feature in individuals with moderate-to-very severe chronic obstructive pulmonary disease (COPD) (1). It is related to exercise intolerance, increased health care use and mortality (1). Physical inactivity is most probably its main underlying cause (1). Therefore, exercise-based pulmonary rehabilitation should be part of the integrated care of individuals with COPD (2).

Lower-limb muscle dysfunction occurs frequently in severely dyspneic individuals with COPD (3). Therefore, there is great interest in effective rehabilitative modalities which do not evoke severe dyspnea, such as strength training or transcutaneous neuromuscular electrical stimulation (NMES) (2). Indeed, the metabolic load on the impaired respiratory system is relatively low during strength training or NMES in individuals with COPD $(4,5)$.

Strength training or high-frequency NMES (HF-NMES; stimulation frequencies $\geq 50$ Hertz $(\mathrm{Hz})$ ) increases quadriceps muscle function, exercise capacity and health status as compared with a non-exercise control group or sham NMES in individuals with COPD, respectively $(6,7)$. To date, a head-to-head comparison of strength training and HF-NMES has not been done in severely dyspneic individuals with COPD. Therefore, it remains unknown whether, and to what extent, these training modalities may have similar effects in individuals with COPD. A priori, no differences were expected between the groups.

Low-frequency NMES (LF-NMES, at $15 \mathrm{~Hz}$ ) of lower-limb muscles increased exercise capacity in individuals with chronic heart failure (8). To date, the effects of LF-NMES at $15 \mathrm{~Hz}$ have not been studied in individuals with COPD. However, it seems reasonable to hypothesize that quadriceps muscle endurance will improve to a greater extent after LF-NMES $(8,9)$, while quadriceps muscle strength will improve to a greater extent after HF-NMES (10).

The aim of the DICES (Dyspneic Individuals with COPD: Electrical stimulation or Strength training) trial was to study the efficacy of HF-NMES, LF-NMES or strength training in severely dyspneic individuals with COPD with quadriceps muscle weakness at baseline.

\section{METHODS}

Please see supplemental material for all details. 


\section{Participants}

Individuals with COPD referred for a pulmonary rehabilitation program at $\mathrm{CIRO}+$ were screened for eligibility (11). Inclusion criteria were primary diagnosis of COPD; baseline modified MRC dyspnea grade 3 or 4 ; and quadriceps weakness ( $\leq 80 \%$ predicted) (12). Exclusion criteria were neuromuscular diseases; joint disorders in hip, leg and/or knee; metal implants in hip, leg and/or knee; cardiac pacemaker or internal cardiac defibrillator; and/or outpatient pulmonary rehabilitation program.

\section{DESIGN AND PROCEDURES}

A prospective, single-blind, randomized controlled trial was designed which was approved by the Medical Ethical Committee of the Maastricht University Medical Centre+ (MEC 09-3-072) and conformed to the principles outlined in the World Medical Association declaration of Helsinki which is revised in Seoul. The DICES trial was registered at http://www.trialregister.nl (NTR2322) before enrolment of the first subject. All participants gave written informed consent to participate. Some baseline findings of the DICES trial have been published (13).

\section{INTERVENTIONS}

Participants were randomly assigned to HF-NMES, LF-NMES, or strength training ( 8 weeks, twice per day, five times per week). All sessions were supervised by a physiotherapist. Symptom scores for dyspnea, fatigue and muscle pain were assessed before and directly after each session (14). Dyspnea and fatigue were assessed using the 10-point Borg scale, where " 0 " means no dyspnea/fatigue at all and " 10 " is the worst dyspnea/fatigue one can imagine (14). The DICES trial was part of a regular eightweek inpatient pulmonary rehabilitation program, including also non-exercising components like occupational therapy, exacerbation management strategies, educational sessions, and psychosocial counselling (2). The interdisciplinary treatment was comparable among the groups. None of the participants underwent treadmill walking or stationary ergometry cycling.

\section{NMES PROTOCOLS}

Quadriceps and calf muscles of both legs were stimulated electrically with a portable battery-operated electrical stimulator, using eight carbon-rubber electrodes (Tensmed S84, Enraf-Nonius, Rotterdam, the Netherlands) (Supplemental Figure S7.1) (5). After a continuous 3-minute warm-up at $5 \mathrm{~Hz}$, intensity was adjusted to individual toleration during each 18-minute session. The frequencies used were $75 \mathrm{~Hz}$ (HF-NMES) or $15 \mathrm{~Hz}$ (LF-NMES) (5). 


\section{StRENGTH TRAINING}

Strength training consisted of bilateral leg extension and bilateral leg press exercises (Technogym SpA, Gambettola, Italy) (15). Both exercises started at $70 \%$ of onerepetition maximum (1RM), four sets of eight repetitions per exercise with at least 2 minutes of recovery between each set. The training load was set to increase with $5 \%$ every two weeks (15).

\section{Outcomes}

\section{Primary outcome}

The primary outcome was the change in isokinetic quadriceps muscle function (i.e. peak muscle strength and endurance), using a Biodex (Biodex System 4 Pro, Biodex Medical Systems, Inc., New York, USA) (16). To minimize learning effects, the measurement was performed twice at the initial assessment. Best values were used for analyses.

\section{Secondary outcomes}

Functional exercise performance was measured with the 6-minute walk test (6MWT), including a practice walk at initial assessment (17). The best value was used for further analyses. The constant work-rate cycling endurance test (CWRT, expressed in seconds) was performed at $75 \%$ of the measured peak cycling work rate (18). Dyspnea and fatigue were assessed before and after exercise tests, and at isotime during the postintervention CWRT. Anxiety and depression were assessed using the Hospital Anxiety Depression Scale (HADS) (19). Disease-specific health status was measured using the St. George's Respiratory Questionnaire (SGRQ) (20). The Canadian Occupational Performance Measure (COPM), a semi-structured interview performed by an occupational therapist, was used to assess problematic activities of daily life (ADLs) on a 10-point scale (21), and has shown to be reliable in individuals with COPD (22). Wholebody dual-energy x-ray absorptiometry scan (DEXA scan) was used to assess body mass index (BMI) and fat-free mass index (FFMI) (23). A modified MRC dyspnea scale was used to assess dyspnea (24). In addition, age, sex, height, weight, comorbidities (25), pulmonary function parameters and arterial blood gases were collected at the initial assessment (13).

\section{SAMPLE SIZE CALCULATION, RANDOMIZATION, BLINDING, AND STATISTICS}

The DICES trial was powered to detect a significant difference in the change in maximal quadriceps strength between HF-NMES and LF-NMES of $9.2 \mathrm{~kg}$ on average (26). Each intervention group needed to have 36 individuals with COPD. Adjusting for drop out and withdrawals, a minimum of 40 individuals per intervention group was aimed for. A randomization schedule was generated by the computer for participants with and without the use of long-term oxygen therapy; and with or without a hospitalization for 
a COPD exacerbation <3 months of enrolment. The sequence was concealed. Analyses were performed using SPSS for Windows, Version 17.0.1 (SPSS, Inc., Chicago, II, USA). Differences within groups were analyzed using paired T-tests or Wilcoxon signed rank test. Groups were compared using an analysis of variance (one-way ANOVA), $\chi^{2}$ test, Fisher's exact test or Kruskal-Wallis one-way analysis of variance, as appropriate. The Bonferroni T-test was used as Post-Hoc test. Correlation analyses were done using Pearson's or Spearman's correlations. The level of significance was set at $\leq 0.05$.

\section{RESULTS}

\section{Participants' flow}

Between September 2010 and November 2012, 120 individuals with COPD were included in the DICES trial (HF-NMES: $n=41$; LF-NMES: $n=39$; strength training: $n=40$ ). Ninety-one individuals (75.8\%) were followed up at 8 weeks. The main reasons for dropping out were severe health problems, including hospital admission. Dropout rates were similar amongst intervention groups (Figure 7.1).

\section{BASELINE CHARACTERISTICS}

Participants generally had severe to very severe COPD, a poor diffusing capacity, explicit quadriceps muscle weakness, very severe dyspnea, a poor functional and peak exercise performance, and a poor health status (Table 7.1 and Supplemental Tables S7.3 and S7.4). Participants used $10 \pm 1$ types of medications (Supplemental Table S7.1), and had 3.2 \pm 0.1 objectified comorbidities (Supplemental Figure 7.2). The 29 individuals who dropped out had similar baseline characteristics as the 91 participants who completed the 8-week intervention (Supplemental Table 7.2).

\section{COURSE OF THE 8-WEEK NMES OR STRENGTH TRAINING}

The 91 participants who completed the program attended $76 \pm 3$ HF-NMES sessions, $74 \pm 1$ LF-NMES sessions, or $68 \pm 2$ strength training sessions (both NMES interventions versus strength training, $p<0.001)$. Exacerbations requiring antibiotics and/or corticosteroid treatment occurred frequently during all three interventions: $58 \%$ of the HF-NMES participants; $41 \%$ of the LF-NMES participants; and $41 \%$ of the strength training participants $(p=0.254)$. During exacerbations of COPD which did not require hospital admission, NMES sessions (HF and LF) could be continued. Strength training sessions were not always possible during exacerbations. The mean numbers of training sessions did not differ between the patients with and without $\geq 1$ exacerbations $(p=0.564)$. 
Table 7.1 General characteristics.

\begin{tabular}{|c|c|c|c|c|c|}
\hline & $\begin{array}{c}\text { Total group } \\
n=120\end{array}$ & $\begin{array}{c}\text { HF-NMES } \\
n=41\end{array}$ & $\begin{array}{c}\text { LF-NMES } \\
n=39\end{array}$ & $\begin{array}{l}\text { Strength training } \\
n=40\end{array}$ & P-value \\
\hline Sex (M/F) & $62 / 58$ & $24 / 17$ & $19 / 20$ & $19 / 21$ & 0.555 \\
\hline Age (years) & $64.8 \pm 0.8$ & $64.4 \pm 1.3$ & $66.2 \pm 1.3$ & $64.0 \pm 1.3$ & 0.440 \\
\hline \multicolumn{6}{|l|}{ Pulmonary function } \\
\hline $\mathrm{FEV}_{1}$ (liters) & $0.85 \pm 0.03$ & $0.87 \pm 0.04$ & $0.87 \pm 0.07$ & $0.80 \pm 0.05$ & 0.578 \\
\hline $\mathrm{FEV}_{1}(\%$ predicted) & $33 \pm 1$ & $33 \pm 2$ & $35 \pm 2$ & $33 \pm 2$ & 0.645 \\
\hline $\mathrm{FEV}_{1} / \mathrm{VC} \max (\%)$ & $32 \pm 1$ & $31 \pm 1$ & $31 \pm 2$ & $33 \pm 2$ & 0.545 \\
\hline $\mathrm{DL}_{\mathrm{co}}(\%$ predicted $)$ & $41 \pm 1$ & $39 \pm 2$ & $43 \pm 2$ & $42 \pm 3$ & 0.558 \\
\hline LTOT (\%) & $5 \overline{1}$ & $5 \overline{6}$ & $5 \overline{4}$ & $4 \overline{3}$ & 0.429 \\
\hline \multicolumn{6}{|l|}{ (I/II/III/IV) } \\
\hline \multicolumn{6}{|l|}{$(A / B / C / D)$} \\
\hline BMI $\left(\mathrm{kg} / \mathrm{m}^{2}\right)$ & $24.8 \pm 0.5$ & $24.1 \pm 0.8$ & $25.5 \pm 0.8$ & $24.9 \pm 0.8$ & 0.441 \\
\hline FFMI $\left(\mathrm{kg} / \mathrm{m}^{2}\right)$ & $16.5 \pm 0.2$ & $16.3 \pm 0.3$ & $16.6 \pm 0.3$ & $16.6 \pm 0.4$ & 0.688 \\
\hline $\begin{array}{l}\text { Isokinetic quadriceps muscle } \\
\text { function }\end{array}$ & $n=\overline{120}$ & $\mathrm{n}=\overline{4} 1$ & $n=\overline{3} 9$ & $n=\overline{4} 0$ & \\
\hline Peak torque (Nm) & $76.2 \pm 2.4$ & $78.7 \pm 4.4$ & $76.1 \pm 4.1$ & $73.4 \pm 4.1$ & 0.682 \\
\hline Peak torque (\% predicted) & $54 \pm 1$ & $54 \pm 3$ & $55 \pm 2$ & $53 \pm 3$ & 0.812 \\
\hline Total work (joules) & $1175 \pm 44$ & $1189 \pm 87$ & $1164 \pm 67$ & $1175 \pm 76$ & 0.975 \\
\hline 6-minute walk test & $n=120$ & $n=\overline{41}$ & $n=39$ & $\mathrm{n}=\overline{40}$ & \\
\hline 6MWD (meters) & $322 \pm 8$ & $311 \pm 16$ & $315 \pm 14$ & $337 \pm 14$ & 0.412 \\
\hline 6MWD (\% predicted) & $52 \pm 1$ & $48 \pm 3$ & $52 \pm 3$ & $54 \pm 3$ & 0.204 \\
\hline Cardiopulmonary exercise test & $\mathrm{n}=\overline{104}$ & $\mathrm{n}=\overline{3} 5$ & $n=\overline{3} 3$ & $n=\overline{3} 6$ & \\
\hline Peak load (watts) & $44 \pm 1$ & $45 \pm 2$ & $45 \pm 2$ & $44 \pm 2$ & 0.984 \\
\hline Peak load (\% predicted) & $40 \pm 2$ & $33 \pm 3$ & $44 \pm 3$ & $44 \pm 5$ & 0.083 \\
\hline Peak $\mathrm{VO}_{2}(\mathrm{ml} / \mathrm{min})$ & $820 \pm 21$ & $831 \pm 37$ & $829 \pm 43$ & $806 \pm 30$ & 0.858 \\
\hline Peak $\mathrm{VO}_{2}(\%$ predicted $)$ & $58 \pm 5$ & $46 \pm 5$ & $57 \pm 6$ & $68 \pm 11$ & 0.179 \\
\hline Peak VE (\%MVV) & $94 \pm 4$ & $91 \pm 6$ & $93 \pm 6$ & $97 \pm 7$ & 0.790 \\
\hline Peak HR (\% predicted) & $75 \pm 1$ & $74 \pm 1$ & $75 \pm 3$ & $75 \pm 1$ & 0.830 \\
\hline $\begin{array}{l}\text { Constant work-rate cycling } \\
\text { endurance test }\end{array}$ & $n=\overline{9} 6$ & $n=\overline{3} 3$ & $n=30$ & $n=33$ & \\
\hline Cycle time (seconds) & $191 \pm 10$ & $199 \pm 20$ & $188 \pm 15$ & $185 \pm 14$ & 0.836 \\
\hline Hospital Anxiety and Depression & $\mathrm{n}=\overline{112}$ & $n=\overline{3} 9$ & $n=\overline{3} 7$ & $n=\overline{3} 6$ & \\
\hline \multicolumn{6}{|l|}{ Scale } \\
\hline Anxiety (points) & $8.9 \pm 0.4$ & $7.3 \pm 0.7$ & $9.7 \pm 0.7$ & $9.8 \pm 0.6$ & 0.018 \\
\hline Depression (points) & $8.4 \pm 0.4$ & $8.1 \pm 0.5$ & $8.0 \pm 0.7$ & $9.1 \pm 0.7$ & 0.436 \\
\hline St. George's Respiratory & $n=\overline{1} 09$ & $n=38$ & $n=36$ & $n=35$ & \\
\hline \multicolumn{6}{|l|}{ Questionnaire } \\
\hline Symptoms (points) & $66.3 \pm 1.6$ & $66.9 \pm 2.5$ & $67.0 \pm 2.9$ & $65.0 \pm 3.1$ & 0.850 \\
\hline Activity (points) & $81.7 \pm 1.6$ & $84.4 \pm 2.2$ & $80.9 \pm 3.0$ & $79.6 \pm 2.9$ & 0.431 \\
\hline Impact (points) & $53.1 \pm 1.7$ & $50.8 \pm 2.4$ & $52.8 \pm 3.4$ & $55.9 \pm 3.0$ & 0.462 \\
\hline Total score (points) & $63.9 \pm 1.3$ & $63.6 \pm 1.7$ & $63.7 \pm 2.6$ & $64.6 \pm 2.3$ & 0.932 \\
\hline
\end{tabular}

Values expressed as mean \pm SEM. Cycle tests have not been performed by all subjects with as major reasons unstable blood gases or severe disabled condition. The major reason for not performing questionnaires are technical problems. Abbreviations: HF-NMES=High-frequency transcutaneous neuromuscular electrical stimulation; LF-NMES=Low-frequency transcutaneous neuromuscular electrical stimulation; $M=m a l e s ;$ $\mathrm{F}=$ females; $\mathrm{FEV}_{1}=$ forced expiratory volume in one second; $\mathrm{VC}$ max=maximum vital capacity; $\mathrm{DL}_{\mathrm{co}}=$ diffusion capacity of the lung for carbon monoxide; LTOT=long-term oxygen therapy; GOLD=Global Initiative for chronic Obstructive Lung Disease; $B M I=$ body mass index; $F F M I=f a t$ free mass index; $\mathrm{kg} / \mathrm{m}^{2}=\mathrm{kilogram}$ per square meter; $\mathrm{FFM}=\mathrm{fat}$ free mass; $6 \mathrm{MWD}=6$-minute walk distance; $\mathrm{VO}_{2}=$ oxygen uptake; $\mathrm{tSaO}_{2}=$ transcutaneous oxygen saturation; $\mathrm{Nm}=$ newton meter; $\mathrm{ml} / \mathrm{min}=$ milliliters per minute; \% $\mathrm{MVV}=$ percentage maximal voluntary ventilation; $\mathrm{bpm}=$ beats per minute. Please see supplemental Tables S7.3 and S7.4 for more details 
Figure 7.1 Flow diagram of DICES trial.

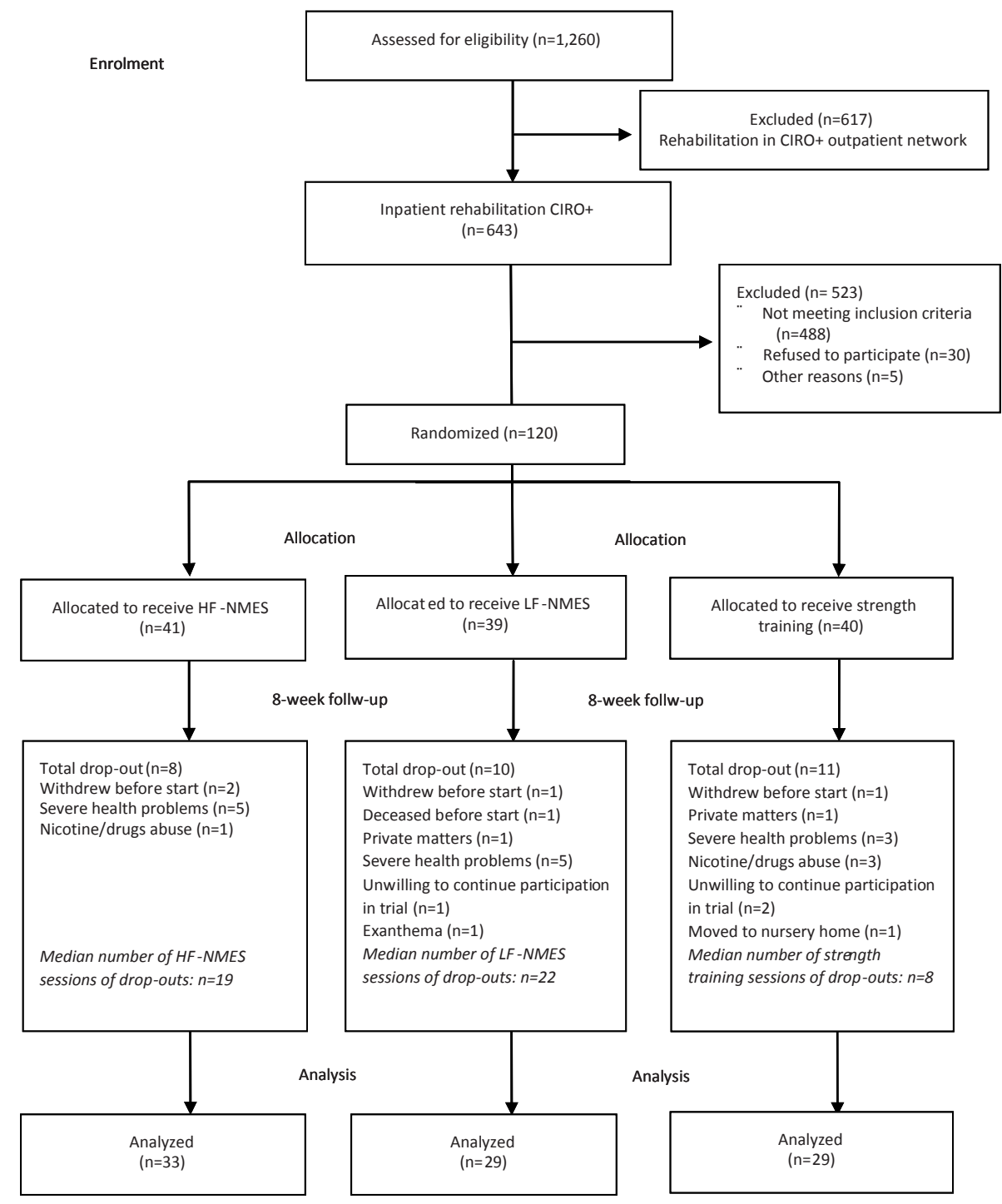

HF/LF-NMES current intensities and strength training loads increased significantly over time (Figure 7.2). All training modalities were safe. No side effects, such as acute dyspnea, or muscle pain, were reported (see Supplemental Figure S7.3). 
Figure 7.2 Change in quadriceps muscle strength and endurance.
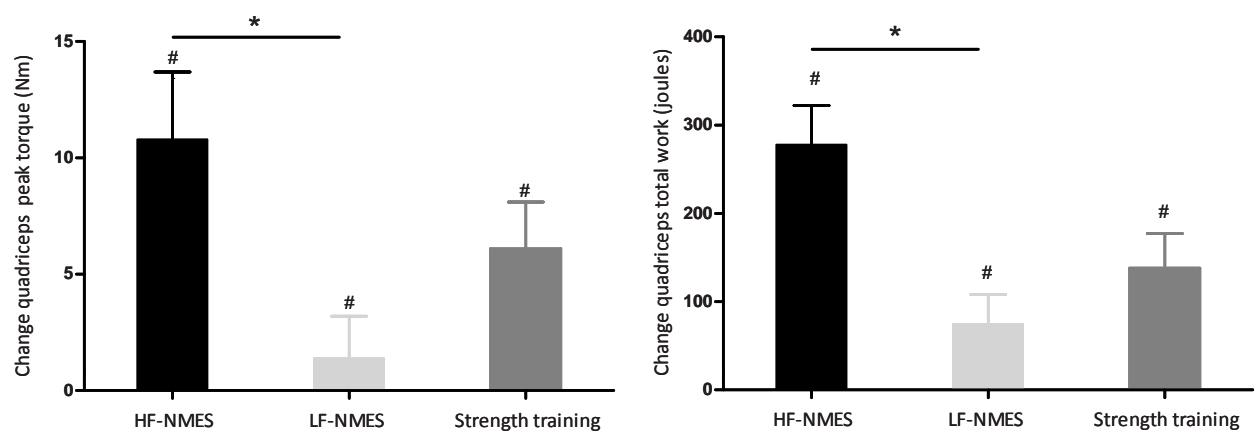

Data shown as mean \pm SEM. HF-NMES=high-frequency transcutaneous neuromuscular electrical stimulation; LF-NMES=low-frequency transcutaneous neuromuscular electrical stimulation. \# $\mathrm{p} \leq 0.05$ compared with baseline- ${ }^{*} \mathrm{p} \leq 0.05$.

\section{Efficacy}

\section{Quadriceps muscle function}

Isokinetic quadriceps peak torque increased significantly after HF-NMES $(10.8 \pm 2.9$ Newton-meter; $p<0.01)$ or strength training $(6.1 \pm 2.0$ Newton-meter; $p<0.01)$, but not after LF-NMES (1.4 \pm 1.8 Newton-meter; $\mathrm{p}=0.43)$. Improvement in isokinetic quadriceps peak torque was significantly higher after HF-NMES compared with LF-NMES $(p=0.01$; Figure 7.2A). Isokinetic total work increased significantly in all groups (HF-NMES: $285 \pm 51$ Joules; LF-NMES: $101 \pm 45$ Joules; strength training: $192 \pm 51$ Joules; all $p<0.03$ ). Again, the improvement after HF-NMES was significantly higher than with LF-NMES $(p=0.03$; Figure 7.2B). Gains in peak muscle strength correlated with the increase in muscle endurance (HF-NMES: $r=0.689$; LF-NMES: $r=0.581$ and strength training: $r=0.689$, all $p<0.001$ ).

\section{Six-minute walk test}

Six-minute walk distance improved in all groups (HF-NMES: $66 \pm 14$ meter; LF-NMES: 51 \pm 15 meter; strength training: $29 \pm 12$ meter; all $p<0.03$; no differences between groups). Interestingly, symptoms at the end of the 6MWT were significantly lower compared than baseline after HF-NMES (dyspnea: $6.9 \pm 0.4$ versus $5.7 \pm 0.4$ points; fatigue: $5.2 \pm 0.5$ versus $3.4 \pm 0.4$ points; both $p<0.014$ ) or LF-NMES (dyspnea: $6.4 \pm 0.4$ versus $5.4 \pm 0.5$ points; fatigue: $4.9 \pm 0.5$ versus $2.8 \pm 0.5$ points; both $p<0.032$ ). Symptoms at the end of the 6-min walk test remained unchanged after strength training (dyspnea: $5.6 \pm 0.4$ versus $5.2 \pm 0.4$ points; fatigue: $4.1 \pm 0.5$ versus $3.3 \pm 0.5$ points; both $p>0.11$ ). 


\section{Constant work-rate test}

Endurance time during the CWRT improved in all groups (HF-NMES: $171 \pm 58$ seconds; LF-NMES: $167 \pm 46$ seconds; strength training: $69 \pm 30$ seconds; all $p<0.03$; no differences between groups). At isotime, symptoms of dyspnea were lower after both NMES protocols, and fatigue was lower after all three interventions compared with the initial constant work-rate test (HF-NMES: $\Delta$ dyspnea isotime: $-1.8 \pm 0.6$ points $(p=0.005)$, $\Delta$ fatigue isotime: $-2.6 \pm 0.6$ points $(p<0.001)$; LF-NMES: $-1.9 \pm 0.6$ points $(p=0.005)$, and $-1.4 \pm 0.5$ points $(p=0.01)$ and strength training: $0.4 \pm 0.4$ points $(p=0.361)$ and $-1.7 \pm 0.5$ points, $(p=0.003)$, respectively. Moreover, symptoms of fatigue at the end of the CWRT were significantly lower compared with baseline after HF-NMES $(6.3 \pm 0.5$ versus $4.7 \pm 0.6$ points), LF-NMES ( $5.8 \pm 0.5$ versus $5.0 \pm 0.6$ points) or strength training $(6.1 \pm 0.5$ versus $4.8 \pm 0.5$ points; all $p<0.05$ ). Symptoms of dyspnea at the end of the cycle endurance test did not change (data not shown).

\section{Body composition}

$\mathrm{BMI}$ and FFMI did not change significantly compared to baseline in any of the groups. Lower-limb FFM increased in all groups (HF-NMES: $0.58 \pm 0.18 \mathrm{~kg}$; LF-NMES: $0.44 \pm 0.18 \mathrm{~kg}$; strength training: $0.37 \pm 0.13 \mathrm{~kg}$; all $\mathrm{p}<0.03$; no differences between groups).

\section{Dyspnea}

Modified MRC dyspnea scores improved in all groups (HF-NMES: $-0.9 \pm 0.2$ points; LF-NMES: $-0.7 \pm 0.2$ points; strength training: $-0.8 \pm 0.2$ points; all $p<0.005$; no differences between groups).

\section{Mood status}

Symptoms of depression improved in all groups (HF-NMES: $-1.8 \pm 0.6$ points; LF-NMES: $-2.2 \pm 0.5$ points; strength training: $-1.6 \pm 0.7$ points; all $p<0.04)$, while symptoms of anxiety only improved following LF-NMES $(-1.8 \pm 0.6$ points; $p=0.004)$. Changes in symptoms of anxiety or depression were similar between groups $(p>0.46)$.

\section{Health status}

Total SGRQ scores improved in all groups compared to baseline (HF-NMES: $-10.2 \pm 2.4$ points; LF-NMES: $-10.5 \pm 3.0$ points; strength training: $-11.4 \pm 2.5$ points; all $p<0.003$ ). Changes in health status scores were similar between groups $(p=0.948)$. See Supplemental Table S7.5 for the significant changes in the SGRQ domain scores.

\section{Problematic activities of daily life}

COPM total scores for problematic activities of daily life improved in all groups compared with baseline (HF-NMES: performance: $2.7 \pm 0.2$ points, satisfaction: $3.5 \pm 0.2$ points; LF-NMES: performance $2.5 \pm 0.2$ points, satisfaction $2.9 \pm 0.3$ points; strength training: performance $2.3 \pm 0.2$ points, satisfaction $2.8 \pm 0.3$ points; $p<0.05$ ), with no 
significant differences between groups. See supplemental Tables S7.6 and S7.7 for details.

\section{DISCUSSION}

As far we know, this is the first trial comparing the effects of HF-NMES, LF-NMES and strength training as the sole supervised muscle training modality during an 8-week pulmonary rehabilitation program in severely dyspneic individuals with COPD and quadriceps weakness at baseline. Lower-limb muscle strength increased in the HFNMES group and the strength training group compared to baseline. Exercise performance, exercise-induced symptoms of dyspnea and fatigue improved significantly compared with baseline in all three lower-limb training modalities. Only the increase in isokinetic quadriceps muscle strength and muscle endurance were higher after HF-NMES than after LF-NMES. Moreover, the DICES trial also showed that symptoms of depression, health status and problematic activities of daily living improved after an interdisciplinary pulmonary rehabilitation program without treadmill walking or stationary cycling.

\section{LOWER-LIMB MUSCLE FUNCTION}

Isokinetic quadriceps muscle strength improved after HF-NMES or strength training. This is in line with previous studies $(6,7)$. Interestingly, no significant differences were found between HF-NMES and strength training. Therefore, HF-NMES may be a preferential muscle training modality in COPD individuals with severe dyspnea and muscle weakness, as the metabolic load on the impaired cardiorespiratory system is significantly lower compared to strength training (4). Moreover, HF-NMES recruits motor units in a non-selective, spatially fixed and temporally synchronous pattern (27) in contrary to the activation order according to the size principle involving the activation of the slower (lower force-producing) motor units before the faster (higher force-producing) units (28). In COPD, atrophy of fast twitch muscle fibers is consistently reported (29). It can be hypothesized that at least some of these fibers can be trained by HF-NMES, whereas these fibers might otherwise only be activated using high-force voluntary efforts (30).

As expected from LF-NMES studies in healthy subjects (31), quadriceps muscle strength did not improve after LF-NMES. Indeed, the change in isokinetic quadriceps muscle strength was significantly higher in HF-NMES group than in the LF-NMES group. Comparable results were found in a study comparing HF-NMES $(50 \mathrm{~Hz})$ with LF-NMES $(15 \mathrm{~Hz})$ in healthy volunteers and in individuals with chronic heart failure (10). The abovementioned results might be obtained because the increase in muscle strength is 
proportional to the stimulation frequency: higher frequencies produce higher torques, which probably result in a greater increase in muscle strength (32). Besides the peripheral pathway, HF-NMES can also produce muscle contractions by central recruitment through the electrically evoked sensory volley in contrast to LF-NMES (33). During HF-NMES, the recruitment of motor units through central pathways can augment contractions generated through peripheral pathways, leading to the development of greater torques (34). While HF-NMES and LF-NMES had differential effects on muscle function in this study, the observed increase in lower-limb muscle mass was comparable, indicating that muscle dysfunction in COPD is not only related to the bulk of muscle.

\section{EXERCISE PERFORMANCE}

This trial shows that lower-limb muscle training modalities improve exercise performance significantly, while it is known that the load on the cardiorespiratory system is rather low $(4,5)$. Indeed, the mean improvement in 6-min walk distance exceeded the minimal important differences of 25 meters in all three intervention groups (35). Even though quadriceps muscle strength and endurance improved to a greater extent after HF-NMES than with LF-NMES, improvements in exercise performance were similar. This might be due to the fact that patients were still limited owing to their dyspnea at the end of the exercise tests, while fatigue symptom scores were clearly lower at the end of the 6MWT or CWRT compared with baseline tests.

\section{DYSPNEA, MOOD STATUS, HEALTH STATUS AND PROBLEMATIC ADL}

Both strength training and HF-NMES have a positive impact on health status in individuals with COPD (7). Unfortunately, the design of our study does not allow us to distinguish between the impact of the muscle training modalities and the nonexercising parts of the pulmonary rehabilitation program. The significant improvements in mood status, health status, and problematic ADLs, however, were very encouraging as clinically relevant thresholds were exceeded, which is also true for walking and/or cycling-based pulmonary rehabilitation programs (2).

\section{StRengthS AND Methodological CONSIDERATIONS}

The DICES trial has several strengths. This is the largest randomized controlled trial studying the efficacy of HF-NMES, LF-NMES or strength training in severely dyspneic individuals with COPD. The number of participants provided enough statistical power to detect possible differences in changes in isokinetic quadriceps muscle function between HF-NMES, LF-NMES and strength training. However, the DICES trial is probably underpowered to detect statistical significant differences between HF/LF-NMES and strength training for changes in exercise performance, which did exceed minimal 
important differences. Isokinetic quadriceps muscle function was assessed twice at the initial assessment to minimize a learning effect. Outcome assessors were blinded for group allocation. They were not (in)directly involved in the delivery of the interventions. This places greater credence in the results (36). Individuals randomly assigned to HF-NMES or LF-NMES were also blinded for the stimulation frequency applied. This is the first trial studying the efficacy of LF-NMES in individuals with COPD. All participants with outcome assessment were analyzed, irrespective of the number of completed sessions or exacerbation treatment with antibiotics and/or corticosteroids during the intervention period.

The DICES trial also had some methodological limitations. The trial design did not include a control group, as strength training or HF-NMES had been proven to be beneficial compared to a non-exercise control group or sham NMES in individuals with COPD, respectively $(6,7,37)$. Twenty-nine participants $(24.2 \%)$ did not complete the DICES trial. Dropping out appeared to be random across the DICES study sample as baseline characteristics were similar between participants who did and did not underwent outcome assessment (Supplemental Table S7.2). We believe that the dropout rates are acceptable, as the DICES sample consisted of severely dyspneic individuals with COPD, who had muscle weakness and multiple coexisting morbidities. Moreover, our drop-out rate is comparable to drop out rates in peer-reviewed COPD strength training trials (range: $11 \%-38 \%(6)$. The generalizability of our findings is limited owing to the strict inclusion and exclusion criteria. This approach, however, is in line with the philosophy of personalized medicine, in which healthcare is tailored to the individual patient or subgroups of patients (38).

\section{Conclusions}

The present study shows that HF-NMES is equally effective as strength training in severely dyspneic individuals with COPD and quadriceps weakness in partially reversing quadriceps muscle dysfunction. HF-NMES may be a good alternative for strength training in this specific patient population. LF-NMES is not effective in improving muscle strength. HF-NMES, LF-NMES and strength training are effective training modalities in improving exercise performance, lower limb fat-free mass and health status in severely dyspneic individuals with COPD and quadriceps weakness. 


\section{REFERENCES}

1. Maltais F, Decramer M, Barreiro E, et al. An Official American Thoracic Society and European Respiratory Society Statement; Update on Limb Muscle Dysfunction in COPD: 2013 Update. Am J Respir Crit Care Med. 2013; Under review.

2. Spruit MA, Singh SJ, Garvey C, et al. An official american thoracic society/european respiratory society statement: key concepts and advances in pulmonary rehabilitation. Am J Respir Crit Care Med. 2013;188(8):e13-64.

3. Seymour JM, Spruit MA, Hopkinson NS, et al. The prevalence of quadriceps weakness in COPD and the relationship with disease severity. Eur Respir J. 2010;36(1):81-8.

4. Sillen MJ, Janssen PP, Akkermans MA, et al. The metabolic response during resistance training and neuromuscular electrical stimulation (NMES) in patients with COPD, a pilot study. Respir Med. 2008;102(5):786-9.

5. Sillen MJ, Wouters EF, Franssen FM, et al. Oxygen uptake, ventilation, and symptoms during lowfrequency versus high-frequency NMES in COPD: a pilot study. Lung. 2011;189(1):21-6.

6. O'Shea SD, Taylor NF, Paratz JD. Progressive resistance exercise improves muscle strength and may improve elements of performance of daily activities for people with COPD: a systematic review. Chest. 2009;136(5):1269-83.

7. Sillen MJ, Speksnijder CM, Eterman RM, et al. Effects of neuromuscular electrical stimulation of muscles of ambulation in patients with chronic heart failure or COPD: a systematic review of the Englishlanguage literature. Chest. 2009;136(1):44-61.

8. Nuhr MJ, Pette D, Berger R, et al. Beneficial effects of chronic low-frequency stimulation of thigh muscles in patients with advanced chronic heart failure. Eur Heart J. 2004;25(2):136-43.

9. Nuhr M, Crevenna R, Gohlsch B, et al. Functional and biochemical properties of chronically stimulated human skeletal muscle. Eur J Appl Physiol. 2003;89(2):202-8.

10. Sbruzzi G, Schaan BD, Pimentel GL, et al. Effects of low frequency functional electrical stimulation with 15 and $50 \mathrm{~Hz}$ on muscle strength in heart failure patients. Disability and rehabilitation. 2011;33(6): 486-93.

11. Spruit MA, Vanderhoven-Augustin I, Janssen PP, et al. Integration of pulmonary rehabilitation in COPD. Lancet. 2008;371(9606):12-3.

12. Borges $\mathrm{O}$. Isometric and isokinetic knee extension and flexion torque in men and women aged 20-70. Scand J Rehabil Med. 1989;21(1):45-53.

13. Sillen MJ, Franssen FM, Delbressine JM, et al. Heterogeneity in clinical characteristics and comorbidities in dyspneic individuals with COPD GOLD D: findings of the DICES trial. Respir Med. 2013;107(8):1186-94.

14. Revill SI, Robinson JO, Rosen $\mathrm{M}$, et al. The reliability of a linear analogue for evaluating pain. Anaesthesia. 1976;31(9):1191-8.

15. Spruit MA, Gosselink R, Troosters $T$, et al. Resistance versus endurance training in patients with COPD and peripheral muscle weakness. Eur Respir J. 2002;19(6):1072-8.

16. Mathur S, Makrides L, Hernandez P. Test-retest reliability of isometric and isokinetic torque in patients with chronic obstructive pulmonary disease. Physiother Can. 2004;56:94-101.

17. Hernandes NA, Wouters EF, Meijer K, et al. Reproducibility of 6-minute walking test in patients with COPD. Eur Respir J. 2011;38(2):261-7.

18. Hul van 't A, Gosselink R, Kwakkel G. Constant-load cycle endurance performance: test-retest reliability and validity in patients with COPD. J Cardiopulm Rehabil. 2003;23(2):143-50.

19. Zigmond AS, Snaith RP. The hospital anxiety and depression scale. Acta Psychiatr Scand. 1983; 67(6):361-70.

20. Jones PW, Quirk FH, Baveystock CM. The St George's Respiratory Questionnaire. Respir Med. 1991;85 Suppl B:25-31; discussion 3-7.

21. Annegarn J, Meijer K, Passos VL, et al. Problematic activities of daily life are weakly associated with clinical characteristics in COPD. J Am Med Dir Assoc. 2012;13(3):284-90.

22. Sewell L, Singh SJ. The Canadian Occupational Performance Measure: is it a reliable measure in clients with chronic obstructive pulmonary disease? Br J Occup Ther. 2001;64:305-10. 
23. Graat-Verboom L, Smeenk FW, van den Borne BE, et al. Progression of osteoporosis in patients with COPD: a 3-year follow up study. Respir Med. 2012;106(6):861-70.

24. Spruit MA, Pennings HJ, Janssen PP, et al. Extra-pulmonary features in COPD patients entering rehabilitation after stratification for MRC dyspnea grade. Respir Med. 2007;101(12):2454-63.

25. Vanfleteren LE, Spruit MA, Groenen M, et al. Clusters of comorbidities based on validated objective measurements and systemic inflammation in patients with chronic obstructive pulmonary disease. Am J Respir Crit Care Med. 2013;187(7):728-35.

26. O'Shea SD, Taylor NF, Paratz JD. A predominantly home-based progressive resistance exercise program increases knee extensor strength in the short-term in people with chronic obstructive pulmonary disease: a randomised controlled trial. Aust J Physiother. 2007;53(4):229-37.

27. Bickel CS, Gregory CM, Dean JC. Motor unit recruitment during neuromuscular electrical stimulation: a critical appraisal. Eur J Appl Physiol. 2011;111(10):2399-407.

28. Henneman E, Somjen G, Carpenter DO. Functional Significance of Cell Size in Spinal Motoneurons. J Neurophysiol. 1965;28:560-80.

29. Gosker HR, Engelen MP, van Mameren $\mathrm{H}$, et al. Muscle fiber type IIX atrophy is involved in the loss of fat-free mass in chronic obstructive pulmonary disease. Am J Clin Nutr. 2002;76(1):113-9.

30. Maffiuletti NA. Physiological and methodological considerations for the use of neuromuscular electrical stimulation. Eur J Appl Physiol. 2010;110(2):223-34.

31. Theriault R, Boulay MR, Theriault G, et al. Electrical stimulation-induced changes in performance and fiber type proportion of human knee extensor muscles. Eur J Appl Physiol Occup Physiol. 1996; 74(4):311-7.

32. Gregory CM, Dixon W, Bickel CS. Impact of varying pulse frequency and duration on muscle torque production and fatigue. Muscle Nerve. 2007;35(4):504-9.

33. Collins DF. Central contributions to contractions evoked by tetanic neuromuscular electrical stimulation. Exerc Sport Sci Rev. 2007;35(3):102-9.

34. Bergquist AJ, Clair JM, Lagerquist $O$, et al. Neuromuscular electrical stimulation: implications of the electrically evoked sensory volley. Eur J Appl Physiol. 2011;111(10):2409-26.

35. Holland $A E$, Hill CJ, Rasekaba $T$, et al. Updating the minimal important difference for six-minute walk distance in patients with chronic obstructive pulmonary disease. Arch Phys Med Rehabil. 2010;91(2):221-5.

36. Schulz KF, Grimes DA. Blinding in randomised trials: hiding who got what. Lancet. 2002;359(9307): 696-700.

37. Simpson K, Killian K, McCartney N, et al. Randomised controlled trial of weightlifting exercise in patients with chronic airflow limitation. Thorax. 1992;47(2):70-5.

38. WIKIPEDIA. http://en.wikipedia.org/wiki/Personalized_medicine. 2013 [13 August 2013]. 


\section{SUPPLEMENTAL MATERIAL}

\section{METHODS}

\section{Participants}

Individuals with COPD referred for an inpatient interdisciplinary pulmonary rehabilitation program at $\mathrm{CIRO}+$, center of expertise for chronic organ failure in Horn (the Netherlands) were screened for eligibility (1). Inclusion criteria were: (i) primary diagnosis of COPD (2); (ii) baseline modified MRC dyspnea grade 3 ("I stop for breath after walking 100 yards or after a few minutes on the level") or 4 ("I am too breathless to leave the house or breathless when dressing or undressing") (3); and (iii) quadriceps weakness (peak torque $\leq 80 \%$ predicted) (4). Exclusion criteria were: (i) neuromuscular diseases; (ii) joint disorders in hip, leg and/or knee; (iii) metal implants in hip, leg and/or knee; (iv) cardiac pacemaker or internal cardiac defibrillator; and/or (v) outpatient pulmonary rehabilitation program.

\section{Design AND PROCEDURES}

A prospective, single-blind, randomized controlled trial was set up according to the Consolidated Standards of Reporting Trials (CONSORT) (5). The DICES (Dyspneic Individuals with COPD: Electrical stimulation or Strength training) trial protocol was approved by the Medical Ethical Committee of the Maastricht University Medical Centre+ (MEC 09-3-072) and conformed to the principles outlined in the World Medical Association declaration of Helsinki which is revised in Seoul (6). Details of the DICES trial were registered at http://www.trialregister.nl (NTR2322) before first subject enrolment. All participants gave written informed consent to participate. Some baseline findings of the DICES trial have been published (7).

\section{INTERVENTIONS}

The DICES trial was part of a regular eight-week inpatient pulmonary rehabilitation program, including also non-exercising components like occupational therapy, exacerbation management strategies, relaxation therapy, educational sessions, and psychosocial counseling (8). The interdisciplinary treatment was comparable amongst groups. None of the participants underwent treadmill walking or stationary ergometry cycling.

Lower-limb muscle training existed of one of the following interventions: HF-NMES; LFNMES; or strength training. The interventions took place in group sessions, twice per day, 5 times per week for 8 weeks. All sessions were supervised by a physiotherapist. 
Symptom scores for dyspnea, fatigue, and muscle pain were assessed before and directly after each session (9).

\section{NMES PROTOCOLS}

NMES involves the application of an electrical current through electrodes placed on the skin over the targeted muscles, thereby depolarizing motor neurons and, in turn, inducing skeletal muscle contractions $(10,11))$. Quadriceps and calf muscles of both legs were stimulated electrically with a portable battery-operated electrical stimulator (Tensmed S84, Enraf-Nonius, Rotterdam, the Netherlands) (Supplemental Figure S7.1). The output characteristics of the device have been checked on an oscilloscope. A total of eight carbon-rubber electrodes in moistened sponges were placed on the target muscles (four electrodes on each leg): two pairs of $8 \times 12 \mathrm{~cm}$ on the quadriceps muscles and two pairs of $4 \times 6 \mathrm{~cm}$ on the calf muscles. The electrodes on the quadriceps muscles were placed transversally $5-10 \mathrm{~cm}$ distal to the inguinal fold and $4-8 \mathrm{~cm}$ proximal to the patella. The electrodes on the calf muscles were placed longitudinally on the belly of the gastrocnemii muscles. Both NMES protocols used a symmetrical biphasic square pulse with pulse duration of $400 \mu \mathrm{s}$. The contraction time was 6 seconds with 8 seconds relaxation excluding 1 second ramp-up and 1 second ramp-down. Thus, the total cycle length was 16 seconds. After a continuous warm-up of 3 minutes at $5 \mathrm{~Hz}$, intensity was adjusted to individual toleration during each session lasting 18 minutes. The frequencies used were $75 \mathrm{~Hz}$ (HF-NMES) or $15 \mathrm{~Hz}$ (LF-NMES) (12).

\section{StRENGTH TRAINING}

Strength training involves exercises that cause muscles to work or hold against an externally applied force or weight (13). Strength training consisted of bilateral leg extension and bilateral leg press exercises (Technogym SpA, Gambettola, Italy) $(14,15)$. The $1 R M$ was determined during the initial assessment to target the training load. Both exercises started at $70 \%$ of 1 -repetition maximum (1RM), 4 sets of 8 repetitions per exercise with at least 2 minutes of recovery between each set. The training load was set to increase with $5 \%$ every two weeks (15).

\section{OUtCOMES}

\section{Primary outcome}

The primary outcome parameter was the change in isokinetic quadriceps muscle function (i.e. peak muscle strength and muscle endurance), using a Biodex (Biodex System 4 Pro, Biodex Medical Systems, Inc., New York, USA) (16). The reliability of this method has been demonstrated previously in patients with COPD (16). To avoid learning effects, the measurement was performed twice at the initial assessment. Best values were used for further analyses. During quadriceps muscle function testing, 
participants were seated upright on the chair of the dynamometer with support of the back and an angle of $90^{\circ}$ of flexion in the hip joint. The participants were secured with straps. The lever arm was attached to the distal part of the tibia and its axis of rotation was aligned with the anatomical axis of the knee joint. Subjects were instructed to keep their hands on their thighs during testing and were asked to perform maximum strength. The participants performed thirty sequential volitional maximal contractions at an angular velocity of $90^{\circ}$ per second. They were strongly encouraged during this isokinetic test. Peak quadriceps muscle strength was defined as the highest peak torque (Newton-meter, $\mathrm{Nm}$ ) and quadriceps muscle endurance as the total amount of delivered energy (Joules, J) in this series of thirty contractions (17).

\section{Secondary outcomes}

Functional exercise performance was measured with the 6-minute walk test (6MWT), including a practice walk at initial assessment (18). The best value was used for further analyses. Moreover, the constant work-rate cycling endurance test (CWRT, expressed in seconds) was performed at $75 \%$ of the measured peak cycling work rate, which has a high reliability in individuals with COPD (19). Symptoms scores for exercise-induced dyspnea and fatigue were assessed before and after these exercise tests.

Symptoms of anxiety and depression were assessed using the Hospital Anxiety Depression Scale (HADS), with scores ranging from 0 (optimal) to 21 points (worst) (20). Disease-specific health status was measured using the St. George's Respiratory Questionnaire (SGRQ) (21).

The Canadian Occupational Performance Measure (COPM), a semi-structured interview performed by an occupational therapist, was used to assess problematic activities of daily life (ADLs) (21) on a 10-point scale, and has been shown to be reliable in individuals with COPD (23).

Whole-body dual-energy $x$-ray absorptiometry scan (DEXA scan) was used to assess body mass index and fat-free mass index (24).

Modified MRC dyspnea scale was used to assess shortness of breath (3). In the modified MRC dyspnea scale patients with COPD have to grade their self-perceived dyspnoea by using pre-defined statements.

\section{SAMPLE SIZE}

The DICES trial was powered to detect a significant difference between the muscle training modality groups of $9.2 \mathrm{~kg}$ on average (25). Based on standard deviations of $14.6 \mathrm{~kg}$ in the intervention group and $13.1 \mathrm{~kg}$ in the control group, a significance level of $5 \%$ and a power of $80 \%$, the number of patients in each intervention group needed to be 36. Adjusting for drop out and withdrawals from the trial, the minimum number of patients to be included in each group was set to be 40 . 


\section{RANDOMization}

The randomization schedule was generated by the computer for participants with and without the use of long-term oxygen therapy; and with or without a hospitalization for a COPD exacerbation < 3 months of enrolment. MAS maintained the randomization schedule centrally, and was not involved in the assessment and treatment of the participants. The sequence was concealed.

\section{BLINDING}

Outcome assessors were blinded for treatment allocation. The investigators supervising the interventions (MJHS, AWV) were blinded for the initial results, and were not involved in the initial or outcome assessments. Participants were instructed to not divulge their group allocation. Participants randomly assigned to one of the NMES groups, were blinded for stimulation frequency.

\section{COMORBIDITIES}

The following comorbidities were objectified, as described before (7):

\section{Body composition abnormalities}

Body mass index (BMI, defined as body weight divided by squared height) and fat-free mass index (FFMI), defined as fat free mass divided by squared height) were determined, and classified as obesity $\left(\mathrm{BMI}>30 \mathrm{~kg} / \mathrm{m}^{2}\right)$, underweight $\left(\mathrm{BMI}<21 \mathrm{~kg} / \mathrm{m}^{2}\right)$, and/or muscle wasting (FFMI $<14.62 \mathrm{~kg} / \mathrm{m}^{2}$ in women and $\mathrm{FFMI}<17.05 \mathrm{~kg} / \mathrm{m}^{2}$ in men) (26). In addition, bone mineral density (BMD of the hip, lumbar spine and whole body region, expressed as T-scores) were determined using dual-energy $x$-ray absorptiometry (24). If the lowest of the three T-scores was <-2.5, the subject was defined as osteoporotic (27).

\section{Symptoms of anxiety and depression}

Symptoms of anxiety and depression were measured using the Hospital Anxiety and Depression Scale (HADS) (20). Scores can range from 0 (optimal) to 21 points (worst). A score of 10 points or more was defined as increased symptoms of anxiety and/or depression $(20,28)$.

\section{Hyperglycemia, anemia, dyslipidemia and systemic inflammation}

Routinely, a post-absorptive venous blood sample was collected from the patients in the fasted state to analyse glucose, hemoglobin, triglycerides, high density lipoprotein (HDL) and creatinine.

A fasting glucose level $>5.6 \mathrm{mmol} / \mathrm{l}$ was defined as hyperglycemia (28); anemia was defined as a hemoglobin level $<13 \mathrm{~g} / \mathrm{dl}(8.1 \mathrm{mmol} / \mathrm{l}$, men) or $<12 \mathrm{~g} / \mathrm{dl}$ (7.5 mmol/l, 
women) (30); dyslipidemia was defined as a triglyceride level above $1.7 \mathrm{mmol} / \mathrm{l}$ or a HDL cholesterol level below $1.03 \mathrm{mmol} / \mathrm{l}$ (men) or below $1.29 \mathrm{mmol} / \mathrm{I}$ (women) (31).

\section{Renal impairment}

Renal function was established by the estimated glomerular filtration rate (eGFR), using the Cockroft-Gault formula (32). Chronic kidney disease was defined as eGFR <60 $\mathrm{ml} / \mathrm{min}$, corresponding with stage 3 chronic kidney disease according to the National Kidney Foundation Kidney Disease Outcome Quality Initiative (NKF KDOQI) guidelines (33).

\section{Cardiovascular abnormalities}

Peripheral blood pressure was measured three times with interval of 5 minutes, after 15 minutes of supine rest in early morning time. Mean values were calculated. Hypertension grade 1 or higher was based on cut-off values of $>140 \mathrm{~mm} \mathrm{Hg}$ for systolic blood pressure and $>90 \mathrm{~mm} \mathrm{Hg}$ for diastolic blood pressure (34).

A resting ECG was obtained and the Cardiac Infarction Injury Score (CIIS) was scored by a cardiologist (NHMKU-L) blinded for medical history and outcome measures. CIIS is an ECG classification system that was developed as a diagnostic tool to determine the presence of myocardial infarctions. It is based on the power of certain electrocardiographic characteristics to discriminate between myocardial infarction patients and healthy individuals. These characteristics are weighted and combined into a single score (35). Myocardial infarction was defined as a CIIS $\geq 20$ (35).

\section{StATISTICAL ANALYSIS}

Analyses were performed using SPSS for Windows, Version 17.0.1 (SPSS, Inc., Chicago, II, USA). Descriptive statistics were presented as means with standard error of the means or numbers with percentages unless otherwise stated. All patients who had their outcome measures assessed were included in the analysis, regardless of the number of sessions they successfully completed. No imputations were made for missing data. Differences within groups were analyzed using paired T-tests or Wilcoxon signed rank test. Groups were compared using an analysis of variance (one-way ANOVA), $\chi^{2}$ test, Fisher's exact test or Kruskal-Wallis one-way analysis of variance, as appropriate. The Bonferroni T-test was used as Post-Hoc test. Correlation analyses were done using Pearson's or Spearman's correlations. The level of significance was set at $\leq 0.05$. 


\section{RESULTS}

\section{Course of the 8-week NMES or strength training}

The quadriceps muscle current intensity increased from $34 \pm 2 \mathrm{~mA}$ (in week 1) to $71 \pm 4 \mathrm{~mA}$ (in week 8) in the HF-NMES group ( $p<0.001$ ); and from $41 \pm 3 \mathrm{~mA}$ to $69 \pm 5 \mathrm{~mA}$ in the LF-NMES group $(p<0.001)$. The calf muscle current intensity increased also during the intervention, but at a lower level (HF-NMES: $26 \pm 1$ to $56 \pm 5 \mathrm{~mA}$; LF-NMES: $34 \pm 2$ to $54 \pm 5 \mathrm{~mA}$; both $\mathrm{p}<0.001)$. The leg extension training load increased from $15 \pm 1$ to $27 \pm 2 \mathrm{~kg}$; and the leg press training load from $38 \pm 4$ to $75 \pm 7 \mathrm{~kg}$ (both $p<0.001$ ). The change in current intensity or training load did not differ between patients with or without exacerbations (all $p>0.07$ ).

Table S7.1 Numbers of patients using various categories of medications.

\begin{tabular}{|c|c|c|}
\hline \multicolumn{2}{|c|}{ Medication } & \multirow{2}{*}{$\begin{array}{l}\mathbf{N} \\
62\end{array}$} \\
\hline 1 & SABA Short acting $\beta 2$-agonists & \\
\hline 2 & SAMA Short-acting anticholinergics (SAAC) & 13 \\
\hline 3 & SABA + SAMA Short-acting combinations (COMBI) & 47 \\
\hline 4 & LABA Long-acting $\beta 2$-agonists & 26 \\
\hline 5 & LAMA Long-acting anticholinergics & 95 \\
\hline 6 & ICS Inhaled corticosteroids alone & 26 \\
\hline 7 & ICS + LABA Inhaled corticosteroids in combination with LABA & 86 \\
\hline 8 & THEOLAIR & 21 \\
\hline 9 & ORAL CORTICOSTEROIDS & 58 \\
\hline 10 & ANTI-LEUKOTRIENES & 3 \\
\hline 11 & ANTIHISTAMINICUM & 8 \\
\hline 12 & NASAL CORTICOSTEROIDS & 1 \\
\hline 13 & ACE OR ARB & 32 \\
\hline 14 & BETA BLOCKERS & 17 \\
\hline 15 & CALCIUM BLOCKERS & 24 \\
\hline 16 & ANTI ARRYTHMICA & 8 \\
\hline 17 & NITRATES & 15 \\
\hline 18 & DIURETICS & 41 \\
\hline 19 & ANTILIPAEMICA & 39 \\
\hline 20 & ANTIAGGREGATES & 36 \\
\hline 21 & COUMARINES & 14 \\
\hline 22 & ORAL ANTIDIABETICA / INSULIN & 11 \\
\hline 23 & CALCIUM SUPPLETION and/or VITAMIN D & 34 \\
\hline 24 & BISFOSFONATES & 39 \\
\hline 25 & ANTIDEPRESSIVES & 24 \\
\hline 26 & ANXIOLYTICS and SLEEP MEDICATION & 39 \\
\hline $27 a$ & PARACETAMOL & 14 \\
\hline $27 b$ & NSAIDs & 12 \\
\hline $27 c$ & MORPHINE & 7 \\
\hline $27 d$ & CODEINE & 10 \\
\hline $27 e$ & OTHER PAINKILLERS & 0 \\
\hline 28 & PPI/ANTACIDA & 73 \\
\hline 29 & ANTIBIOTICS & 21 \\
\hline 30 & ACETYLCYSTEIN & 32 \\
\hline 31 & OTHER MEDICATION & 52 \\
\hline
\end{tabular}


Table S7.2 Characteristics of analyzed group and drop-outs.

\begin{tabular}{|c|c|c|c|c|}
\hline & & $\begin{array}{l}\text { Analyzed group } \\
\qquad n=91\end{array}$ & $\begin{array}{c}\text { Drop out } \\
n=29\end{array}$ & P-value \\
\hline Gender & Male/Female & $44 / 47$ & $18 / 11$ & 0.200 \\
\hline Age & years & $64.3 \pm 0.8$ & $66.7 \pm 1.8$ & 0.163 \\
\hline $\mathrm{FEV}_{1}$ & liters & $0.82 \pm 0.03$ & $0.95 \pm 0.08$ & 0.089 \\
\hline $\mathrm{FEV}_{1}$ & $\%$ predicted & $33 \pm 1$ & $36 \pm 3$ & 0.315 \\
\hline $\mathrm{FEV}_{1} / \mathrm{VC} \max$ & $\%$ & $32 \pm 1$ & $31 \pm 2$ & 0.721 \\
\hline $\mathrm{DL}_{\mathrm{CO}}$ & $\%$ & $41 \pm 2$ & $41 \pm 3$ & 0.960 \\
\hline RV & $\%$ & $197 \pm 6$ & $203 \pm 11$ & 0.641 \\
\hline $\mathrm{PaO}_{2}$ & $\mathrm{kPa}$ & $9.6 \pm 0.2$ & $10.0 \pm 0.3$ & 0.257 \\
\hline $\mathrm{PaCO}_{2}$ & $\mathrm{kPa}$ & $5.7 \pm 0.1$ & $5.6 \pm 0.2$ & 0.913 \\
\hline $\mathrm{SaO}_{2}$ & $\%$ & $95.2 \pm 0.2$ & $95.5 \pm 0.5$ & 0.845 \\
\hline Peak load & watts & $44 \pm 1$ & $45 \pm 2$ & 0.773 \\
\hline Peak load & $\%$ predicted & $42 \pm 3$ & $37 \pm 3$ & 0.367 \\
\hline Peak $\mathrm{VO}_{2}$ & $\mathrm{ml} / \mathrm{min}$ & $824 \pm 25$ & $811 \pm 38$ & 0.787 \\
\hline Peak VE & liters & $34 \pm 1$ & $34 \pm 2$ & 0.837 \\
\hline Cycle time & seconds & $194 \pm 12$ & $182 \pm 16$ & 0.593 \\
\hline 6MWD & meters & $320 \pm 10$ & $323 \pm 16$ & 0.879 \\
\hline 6MWD & $\%$ predicted & $52 \pm 2$ & $52 \pm 2$ & 0.983 \\
\hline Bodyweight & $\mathrm{kg}$ & $69.8 \pm 1.6$ & $67.8 \pm 2.5$ & 0.517 \\
\hline BMI & $\mathrm{kg} / \mathrm{m}^{2}$ & $25.1 \pm 0.5$ & $23.9 \pm 0.8$ & 0.274 \\
\hline FFMI & $\mathrm{kg} / \mathrm{m}^{2}$ & $16.6 \pm 0.2$ & $16.2 \pm 0.3$ & 0.350 \\
\hline Peak torque & $\mathrm{Nm}$ & $76.3 \pm 2.9$ & $77.8 \pm 4.6$ & 0.792 \\
\hline Peak torque & $\%$ predicted & $55 \pm 2$ & $54 \pm 3$ & 0.810 \\
\hline Total work & joules & $1172 \pm 52$ & $1193 \pm 85$ & 0.837 \\
\hline HADS anxiety & points & $8.8 \pm 0.5$ & $9.3 \pm 0.9$ & 0.624 \\
\hline HADS depression & points & $8.6 \pm 0.4$ & $7.4 \pm 0.7$ & 0.168 \\
\hline SGRQ total score & points & $65.0 \pm 1.3$ & $59.4 \pm 3.6$ & 0.081 \\
\hline
\end{tabular}

Values expressed as mean \pm SEM. $F E V_{1}=$ forced expiratory volume in one second; $V C$ max=maximum vital capacity; $\mathrm{DL}_{\mathrm{CO}}=$ diffusion capacity of the lung for carbon monoxide; $\mathrm{RV}=$ residual volume; $\mathrm{PaO}_{2}=$ resting arterial oxygen tension; $\mathrm{PaCO}_{2}=$ resting arterial carbon dioxide tension; $\mathrm{SaO}_{2}=$ resting arterial oxygen tension; peak $\mathrm{VO}_{2}=$ peak oxygen uptake; peak $\mathrm{VE}=$ peak minute ventilation; $6 \mathrm{MWD}=6$-minute walk distance; $\mathrm{BMI}=$ body mass index; $\mathrm{FFMl}=$ fat-free mass index; $\mathrm{Nm}=$ newtonmeter; $\mathrm{kPa}=\mathrm{kilopascal;} \mathrm{ml} / \mathrm{min}=$ milliliter per minute; $\mathrm{kg} / \mathrm{m}^{2}=$ kilogram per squared meter. 
Table S7.3 General characteristics.

\begin{tabular}{|c|c|c|c|c|c|}
\hline & $\begin{array}{c}\text { Total group } \\
n=120\end{array}$ & $\begin{array}{c}\text { HF-NMES } \\
n=41\end{array}$ & $\begin{array}{c}\text { LF-NMES } \\
n=39\end{array}$ & $\begin{array}{l}\text { Strength training } \\
\mathrm{n}=40\end{array}$ & P-value \\
\hline $\operatorname{Sex}(M / F)$ & $62 / 58$ & $24 / 17$ & $19 / 20$ & $19 / 21$ & 0.555 \\
\hline Age (years) & $64.8 \pm 0.8$ & $64.4 \pm 1.3$ & $66.2 \pm 1.3$ & $64.0 \pm 1.3$ & 0.440 \\
\hline \multicolumn{6}{|l|}{ Pulmonary function } \\
\hline $\mathrm{FEV}_{1}$ (liters) & $0.85 \pm 0.03$ & $0.87 \pm 0.04$ & $0.87 \pm 0.07$ & $0.80 \pm 0.05$ & 0.578 \\
\hline $\mathrm{FEV}_{1}(\%$ predicted) & $33 \pm 1$ & $33 \pm 2$ & $35 \pm 2$ & $33 \pm 2$ & 0.645 \\
\hline $\mathrm{FEV}_{1} / \mathrm{VC} \max (\%)$ & $32 \pm 1$ & $31 \pm 1$ & $31 \pm 2$ & $33 \pm 2$ & 0.545 \\
\hline $\mathrm{DL}_{\mathrm{co}}$ (\% predicted) & $41 \pm 1$ & $39 \pm 2$ & $43 \pm 2$ & $42 \pm 3$ & 0.558 \\
\hline RV (\% predicted) & $198 \pm 5$ & $197 \pm 9$ & $194 \pm 10$ & $206 \pm 9$ & 0.590 \\
\hline \multicolumn{6}{|l|}{ Arterial blood gases } \\
\hline $\mathrm{PaO}_{2}(\mathrm{kPa})$ & $9.7 \pm 0.1$ & $9.9 \pm 0.3$ & $9.7 \pm 0.3$ & $9.5 \pm 0.2$ & 0.852 \\
\hline $\mathrm{PaCO}_{2}(\mathrm{kPa})$ & $5.7 \pm 0.1$ & $5.6 \pm 0.2$ & $5.5 \pm 0.2$ & $5.8 \pm 0.2$ & 0.664 \\
\hline $\mathrm{SaO}_{2}(\%)$ & $95.2 \pm 0.2$ & $95.6 \pm 0.3$ & $95.1 \pm 0.4$ & $95.1 \pm 0.4$ & 0.848 \\
\hline LTOT (\%) & 51 & 56 & 54 & 43 & 0.429 \\
\hline $\begin{array}{l}\text { GOLD classification } \\
(\mathrm{I} / \mathrm{II} / \mathrm{III} / \mathrm{IV})\end{array}$ & $0 / 12 / 36 / 72$ & $0 / 5 / 12 / 24$ & $0 / 2 / 14 / 23$ & $0 / 5 / 10 / 25$ & 0.942 \\
\hline GOLD classification (new) & $0 / 3 / 0 / 117$ & $0 / 2 / 0 / 39$ & $0 / 0 / 0 / 39$ & $0 / 1 / 0 / 39$ & 0.380 \\
\hline \multicolumn{6}{|l|}{ (A/B/C/D) } \\
\hline BMI $\left(\mathrm{kg} / \mathrm{m}^{2}\right)$ & $24.8 \pm 0.5$ & $24.1 \pm 0.8$ & $25.5 \pm 0.8$ & $24.9 \pm 0.8$ & 0.441 \\
\hline FFMI $\left(\mathrm{kg} / \mathrm{m}^{2}\right)$ & $16.5 \pm 0.2$ & $16.3 \pm 0.3$ & $16.6 \pm 0.3$ & $16.6 \pm 0.4$ & 0.688 \\
\hline
\end{tabular}

Values expressed as mean \pm SEM, percentages or numbers. HF-NMES=High-frequency transcutaneous neuromuscular electrical stimulation; LF-NMES=Low-frequency transcutaneous neuromuscular electrical stimulation; $\mathrm{M}=$ males; $\mathrm{F}=$ females; $\mathrm{FEV}_{1}=$ forced expiratory volume in one second; $\mathrm{VC}$ max=maximum vital capacity; $\mathrm{DL}_{\mathrm{CO}}=$ diffusion capacity of the lung for carbon monoxide; $\mathrm{RV}=$ residual volume; $\mathrm{PaO}_{2}=$ resting arterial oxygen tension; $\mathrm{PaCO}_{2}=$ resting arterial carbon dioxide tension; $\mathrm{SaO}_{2}=$ resting arterial oxygen tension; $\mathrm{kPa}=$ kilopascal; LTOT=long-term oxygen therapy; GOLD=Global Initiative for chronic Obstructive Lung Disease; $\mathrm{BMI}=$ body mass index; $\mathrm{FFMI}=$ fat free mass index; $\mathrm{kg} / \mathrm{m}^{2}=\mathrm{kilogram}$ per square meter. 
Table S7.4 Baseline lower-limb muscle function, exercise performance, HADS and SGRQ.

\begin{tabular}{|c|c|c|c|c|c|}
\hline & Total group & HF-NMES & LF-NMES & $\begin{array}{c}\text { Strength } \\
\text { training }\end{array}$ & P-value \\
\hline $\begin{array}{l}\text { Isokinetic quadriceps muscle } \\
\text { function }\end{array}$ & $n=120$ & $n=41$ & $n=39$ & $\mathrm{n}=40$ & \\
\hline Peak torque (Nm) & $76.2 \pm 2.4$ & $78.7 \pm 4.4$ & $76.1 \pm 4.1$ & $73.4 \pm 4.1$ & 0.682 \\
\hline Peak torque (\% predicted) & $54 \pm 1$ & $54 \pm 3$ & $55 \pm 2$ & $53 \pm 3$ & 0.812 \\
\hline Total work (joules) & $1175 \pm 44$ & $1189 \pm 87$ & $1164 \pm 67$ & $1175 \pm 76$ & 0.975 \\
\hline 6-minute walk test & $n=120$ & $n=41$ & $\mathrm{n}=39$ & $n=40$ & \\
\hline 6MWD (meters) & $322 \pm 8$ & $311 \pm 16$ & $315 \pm 14$ & $337 \pm 14$ & 0.412 \\
\hline 6MWD (\% predicted) & $52 \pm 1$ & $48 \pm 3$ & $52 \pm 3$ & $54 \pm 3$ & 0.204 \\
\hline Dyspnea, end (points) & $6.4 \pm 0.2$ & $6.7 \pm 0.4$ & $6.5 \pm 0.3$ & $5.8 \pm 0.3$ & 0.126 \\
\hline Fatigue, end (points) & $4.9 \pm 0.2$ & $5.2 \pm 0.4$ & $5.4 \pm 0.5$ & $4.0 \pm 0.4$ & 0.048 \\
\hline Saturation, end (\%) & $86.6 \pm 0.6$ & $87.3 \pm 1.0$ & $86.5 \pm 1.1$ & $86.1 \pm 1.0$ & 0.687 \\
\hline Cardiopulmonary exercise test & $n=104$ & $\mathrm{n}=35$ & $n=33$ & $n=36$ & \\
\hline Peak load (watts) & $44 \pm 1$ & $45 \pm 2$ & $45 \pm 2$ & $44 \pm 2$ & 0.984 \\
\hline Peak load (\% predicted) & $40 \pm 2$ & $33 \pm 3$ & $44 \pm 3$ & $44 \pm 5$ & 0.083 \\
\hline Peak $\mathrm{VO}_{2}$ (ml/min) & $820 \pm 21$ & $831 \pm 37$ & $829 \pm 43$ & $806 \pm 30$ & 0.858 \\
\hline Peak $\mathrm{VO}_{2}(\%$ predicted) & $58 \pm 5$ & $46 \pm 5$ & $57 \pm 6$ & $68 \pm 11$ & 0.179 \\
\hline Peak VE (liters) & $34 \pm 5$ & $33 \pm 2$ & $34 \pm 2$ & $33 \pm 2$ & 0.993 \\
\hline Peak VE (\%MVV) & $94 \pm 4$ & $91 \pm 6$ & $93 \pm 6$ & $97 \pm 7$ & 0.790 \\
\hline Peak HR (bpm) & $114 \pm 1$ & $114 \pm 2$ & $110 \pm 3$ & $117 \pm 3$ & 0.139 \\
\hline Peak HR (\% predicted) & $75 \pm 1$ & $74 \pm 1$ & $75 \pm 3$ & $75 \pm 1$ & 0.830 \\
\hline Dyspnoea, end (points) & $7.3 \pm 0.2$ & $7.1 \pm 0.3$ & $7.4 \pm 0.3$ & $7.3 \pm 0.3$ & 0.794 \\
\hline Fatigue, end (points) & $5.6 \pm 0.3$ & $5.6 \pm 0.4$ & $5.8 \pm 0.5$ & $5.3 \pm 0.4$ & 0.718 \\
\hline Saturation, end (\%) & $91.3 \pm 0.4$ & $91.3 \pm 0.8$ & $91.7 \pm 0.7$ & $91.0 \pm 0.6$ & 0.808 \\
\hline$\Delta \mathrm{tSaO}_{2}(\%)$ & $-2.9 \pm 0.3$ & $-3.0 \pm 0.6$ & $-2.8 \pm 0.6$ & $-2.9 \pm 0.5$ & 0.901 \\
\hline $\begin{array}{l}\text { Constant work-rate cycling } \\
\text { endurance test }\end{array}$ & $n=96$ & $n=33$ & $n=30$ & $n=33$ & \\
\hline Cycle time (seconds) & $191 \pm 10$ & $199 \pm 20$ & $188 \pm 15$ & $185 \pm 14$ & 0.836 \\
\hline Dyspnea, end (points) & $7.1 \pm 0.2$ & $7.1 \pm 0.3$ & $7.2 \pm 0.4$ & $7.0 \pm 0.3$ & 0.900 \\
\hline Fatigue, end (points) & $6.2 \pm 0.2$ & $6.3 \pm 0.4$ & $6.0 \pm 0.4$ & $6.2 \pm 0.4$ & 0.853 \\
\hline Saturation, end (\%) & $90.0 \pm 0.4$ & $90.2 \pm 0.8$ & $91.1 \pm 0.7$ & $88.8 \pm 0.6$ & 0.096 \\
\hline Hospital Anxiety and & $\mathrm{n}=112$ & $n=39$ & $n=37$ & $n=36$ & \\
\hline \multicolumn{6}{|l|}{ Depression Scale } \\
\hline Anxiety (points) & $8.9 \pm 0.4$ & $7.3 \pm 0.7$ & $9.7 \pm 0.7$ & $9.8 \pm 0.6$ & 0.018 \\
\hline Depression (points) & $8.4 \pm 0.4$ & $8.1 \pm 0.5$ & $8.0 \pm 0.7$ & $9.1 \pm 0.7$ & 0.436 \\
\hline St. George's Respiratory & $n=109$ & $n=38$ & $n=36$ & $n=35$ & \\
\hline \multicolumn{6}{|l|}{ Questionnaire } \\
\hline Symptoms (points) & $66.3 \pm 1.6$ & $66.9 \pm 2.5$ & $67.0 \pm 2.9$ & $65.0 \pm 3.1$ & 0.850 \\
\hline Activity (points) & $81.7 \pm 1.6$ & $84.4 \pm 2.2$ & $80.9 \pm 3.0$ & $79.6 \pm 2.9$ & 0.431 \\
\hline Impact (points) & $53.1 \pm 1.7$ & $50.8 \pm 2.4$ & $52.8 \pm 3.4$ & $55.9 \pm 3.0$ & 0.462 \\
\hline Total score (points) & $63.9 \pm 1.3$ & $63.6 \pm 1.7$ & $63.7 \pm 2.6$ & $64.6 \pm 2.3$ & 0.932 \\
\hline
\end{tabular}

Values expressed as mean \pm SEM. Cycle tests have not been performed by all subjects with as major reasons unstable blood gases or severe disabled condition. The major reason for not performing questionnaires are technical problems. HF-NMES=High-frequency transcutaneous neuromuscular electrical stimulation; LFNMES=Low-frequency transcutaneous neuromuscular electrical stimulation; FFM=fat free mass; 6MWD=6minute walk distance; $\mathrm{VO}_{2}=$ oxygen uptake; $\mathrm{tSaO}_{2}=$ transcutaneous oxygen saturation; $\mathrm{Nm}=$ newton meter; $\mathrm{ml} / \mathrm{min}=$ millilitres per minute; $\% \mathrm{MVV}=$ percentage maximal voluntary ventilation; bpm=beats per minute. 
Table S7.5 Health status.

\begin{tabular}{lcccccccccc}
\hline & & \multicolumn{3}{c}{ HF-NMES } & \multicolumn{3}{c}{ LF-NMES } & \multicolumn{3}{c}{ Strength training } \\
& & Baseline & End & P-value & Baseline & End & P-value & Baseline & End & P-value \\
\hline SGRQ & & & $\mathrm{n}=31$ & & & $\mathrm{n}=29$ & & & $\mathrm{n}=28$ & \\
Symptoms & points & $66.8 \pm 3.0$ & $56.4 \pm 3.2$ & 0.012 & $68.6 \pm 2.7$ & $62.6 \pm 2.8$ & 0.028 & $65.0 \pm 3.1$ & $54.2 \pm 4.3$ & 0.019 \\
Activity & points & $84.4 \pm 2.4$ & $76.0 \pm 3.0$ & 0.049 & $83.5 \pm 2.9$ & $75.9 \pm 3.7$ & 0.092 & $82.6 \pm 2.2$ & $73.1 \pm 4.1$ & 0.016 \\
Impact & points & $50.7 \pm 2.8$ & $38.3 \pm 2.7$ & $<0.001$ & $55.2 \pm 3.6$ & $41.2 \pm 3.5$ & 0.001 & $56.3 \pm 3.0$ & $42.3 \pm 3.1$ & 0.001 \\
Total score & points & $63.4 \pm 2.0$ & $52.7 \pm 2.0$ & $<0.001$ & $66.0 \pm 2.6$ & $55.6 \pm 2.6$ & 0.002 & $65.7 \pm 2.1$ & $53.6 \pm 2.7$ & $<0.001$ \\
\hline
\end{tabular}

Values expressed as mean \pm SEM. HF-NMES=high-frequency transcutaneous neuromuscular electrical stimulation; LFNMES=low-frequency transcutaneous neuromuscular electrical stimulation; SGRQ=St. George's Respiratory Questionnaire.

Table S7.6 Canadian Occupational Performance Measure.

\begin{tabular}{|c|c|c|c|c|c|c|c|c|c|c|}
\hline & & \multicolumn{3}{|c|}{$\begin{array}{c}\text { HF-NMES } \\
n=33\end{array}$} & \multicolumn{3}{|c|}{$\begin{array}{c}\text { LF-NMES } \\
n=29\end{array}$} & \multicolumn{3}{|c|}{$\begin{array}{c}\text { Strength training } \\
n=29\end{array}$} \\
\hline & & Baseline & End & P-value & Baseline & End & P-value & Baseline & End & P-value \\
\hline \multicolumn{11}{|l|}{ Domain } \\
\hline \multirow[t]{2}{*}{ Self-care } & $\mathrm{P}$ & $4.2 \pm 0.3$ & $6.8 \pm 0.2$ & $<0.001$ & $3.8 \pm 0.3$ & $6.3 \pm 0.4$ & $<0.001$ & $4.1 \pm 0.3$ & $6.4 \pm 0.3$ & $<0.001$ \\
\hline & $S$ & $3.4 \pm 0.3$ & $6.7 \pm 0.3$ & $<0.001$ & $3.3 \pm 0.4$ & $6.4 \pm 0.4$ & $<0.001$ & $2.8 \pm 0.3$ & $6.1 \pm 0.5$ & $<0.001$ \\
\hline \multirow[t]{2}{*}{ Mobility } & $\mathrm{P}$ & $3.4 \pm 0.2$ & $6.2 \pm 0.3$ & $<0.001$ & $3.6 \pm 0.3$ & $6.2 \pm 0.3$ & $<0.001$ & $3.3 \pm 0.2$ & $6.0 \pm 0.3$ & $<0.001$ \\
\hline & $\mathrm{S}$ & $2.6 \pm 0.2$ & $6.2 \pm 0.4$ & $<0.001$ & $3.4 \pm 0.3$ & $6.3 \pm 0.4$ & $<0.001$ & $2.9 \pm 0.3$ & $6.0 \pm 0.4$ & $<0.001$ \\
\hline \multirow[t]{2}{*}{ Productivity } & $\mathrm{P}$ & $3.8 \pm 0.3$ & $6.2 \pm 0.4$ & $<0.001$ & $3.3 \pm 0.3$ & $5.7 \pm 0.4$ & $<0.001$ & $3.4 \pm 0.3$ & $6.0 \pm 0.4$ & $<0.001$ \\
\hline & $S$ & $2.9 \pm 0.3$ & $6.2 \pm 0.4$ & $<0.001$ & $3.2 \pm 0.4$ & $5.8 \pm 0.4$ & $<0.001$ & $3.2 \pm 0.3$ & $5.8 \pm 0.4$ & $<0.001$ \\
\hline \multirow[t]{2}{*}{ Leisure } & $P$ & $2.4 \pm 0.4$ & $5.8 \pm 0.5$ & $<0.001$ & $4.1 \pm 0.5$ & $5.8 \pm 0.7$ & 0.005 & $4.9 \pm 0.7$ & $6.3 \pm 0.6$ & 0.040 \\
\hline & $\mathrm{S}$ & $2.1 \pm 0.3$ & $6.1 \pm 0.4$ & $<0.001$ & $3.6 \pm 0.5$ & $6.0 \pm 0.8$ & 0.002 & $4.1 \pm 0.7$ & $5.4 \pm 1.0$ & 0.028 \\
\hline \multirow[t]{2}{*}{ Total } & $\mathrm{P}$ & $3.5 \pm 0.1$ & $6.3 \pm 0.2$ & $<0.001$ & $3.7 \pm 0.2$ & $6.0 \pm 0.2$ & $<0.001$ & $3.6 \pm 0.2$ & $6.1 \pm 0.2$ & $<0.001$ \\
\hline & $\mathrm{S}$ & $2.8 \pm 0.2$ & $6.3 \pm 0.2$ & $<0.001$ & $3.4 \pm 0.2$ & $6.1 \pm 0.2$ & $<0.001$ & $3.0 \pm 0.2$ & $5.9 \pm 0.2$ & $<0.001$ \\
\hline
\end{tabular}

Values expressed as mean $\pm \mathrm{SEM}$. HF-NMES=high-frequency transcutaneous neuromuscular electrical stimulation; LFNMES=low-frequency transcutaneous neuromuscular electrical stimulation; $\mathrm{P}=$ performance (points); $\mathrm{S}=$ satisfaction (points).

Table S7.7 Changes in COPM performance and satisfaction scores.

\begin{tabular}{|c|c|c|c|c|c|}
\hline & & $\begin{array}{c}\text { HF-NMES } \\
n=33\end{array}$ & $\begin{array}{c}\text { LF-NMES } \\
n=29\end{array}$ & $\begin{array}{l}\text { Strength training } \\
\mathrm{n}=29\end{array}$ & P-value \\
\hline \multicolumn{6}{|l|}{ Domain } \\
\hline \multirow[t]{2}{*}{ Self care } & $\mathrm{P}$ & $2.6 \pm 0.3$ & $2.5 \pm 0.4$ & $2.4 \pm 0.4$ & 0.876 \\
\hline & $S$ & $3.3 \pm 0.4$ & $3.1 \pm 0.5$ & $3.4 \pm 0.5$ & 0.939 \\
\hline \multirow[t]{2}{*}{ Mobility } & $P$ & $2.8 \pm 0.3$ & $2.8 \pm 0.4$ & $2.5 \pm 0.3$ & 0.852 \\
\hline & $\mathrm{S}$ & $3.6 \pm 0.4$ & $3.0 \pm 0.4$ & $2.8 \pm 0.4$ & 0.359 \\
\hline \multirow[t]{2}{*}{ Productivity } & $\mathrm{P}$ & $2.3 \pm 0.5$ & $2.5 \pm 0.4$ & $2.6 \pm 0.4$ & 0.429 \\
\hline & $S$ & $3.1 \pm 0.6$ & $2.9 \pm 0.5$ & $2.8 \pm 0.5$ & 0.600 \\
\hline \multirow{2}{*}{ Leisure } & $\mathrm{P}$ & $3.1 \pm 0.7$ & $2.6 \pm 0.5$ & $1.9 \pm 0.7$ & 0.280 \\
\hline & $\mathrm{S}$ & $3.7 \pm 0.3$ & $3.3 \pm 0.6$ & $2.1 \pm 0.8$ & 0.256 \\
\hline \multirow[t]{2}{*}{ Total } & $\mathrm{P}$ & $2.7 \pm 0.2$ & $2.5 \pm 0.2$ & $2.3 \pm 0.2$ & 0.609 \\
\hline & $\mathrm{S}$ & $3.5 \pm 0.2$ & $2.9 \pm 0.3$ & $2.8 \pm 0.3$ & 0.155 \\
\hline
\end{tabular}

Values expressed as mean \pm SEM. HF-NMES=high-frequency transcutaneous neuromuscular electrical stimulation; LF-NMES=low-frequency transcutaneous neuromuscular electrical stimulation; $\mathrm{P}=$ performance (points); S=satisfaction (points). 
Figure S7.1 Transcutaneous neuromuscular electrical stimulation of a man with COPD GOLD IV. Written consent was obtained for the use of this photograph.

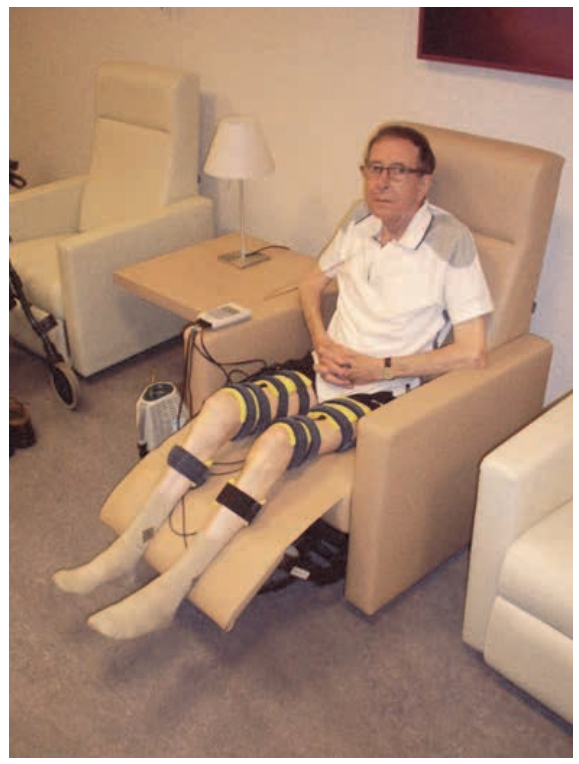

Figure S7.2 Comorbidities.

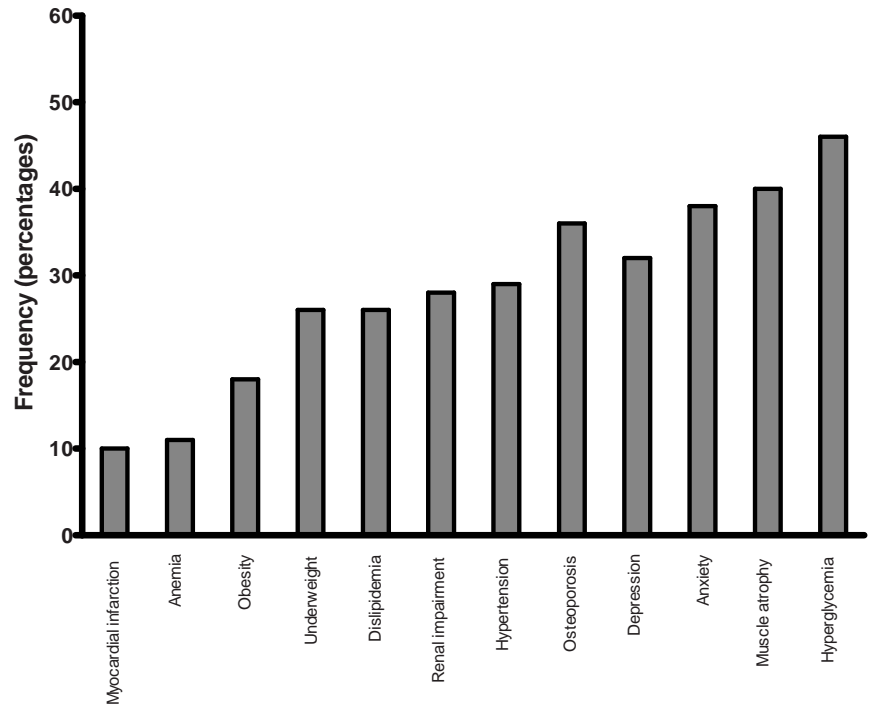


Figure S7.3 End dyspnea scores (A. HF-NMES; B. LF-NMES; C. Strength training), end fatigue scores (D. HF-NMES; E. LF-NMES; F. Strength training) and end muscle pain scores (G. HF-NMES; H. LF-NMES; I. Strength training) directly after the interventions.

\section{A}

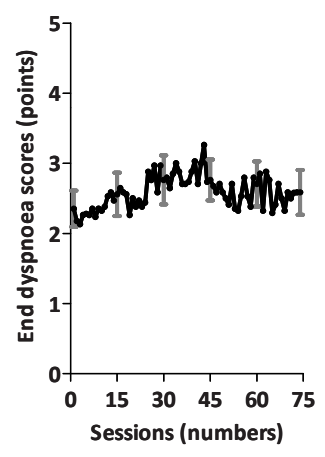

D

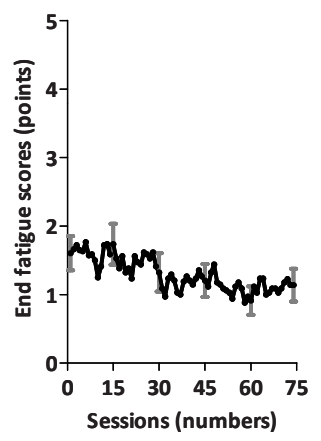

G

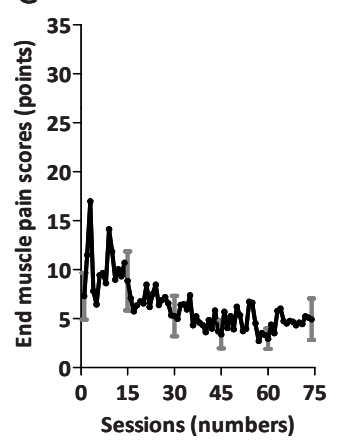

B

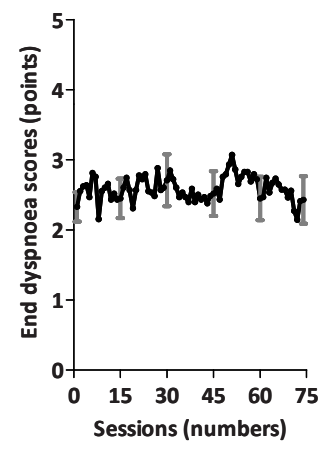

$\mathrm{E}$

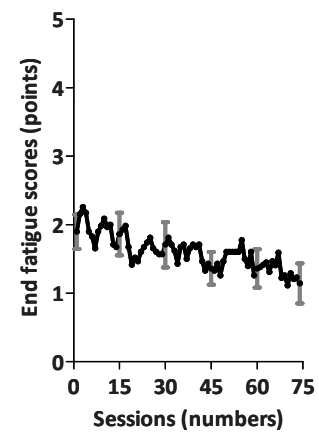

$\mathrm{H}$

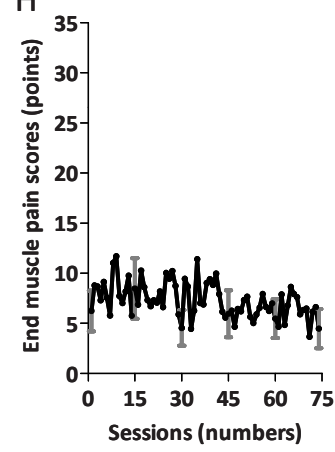

C

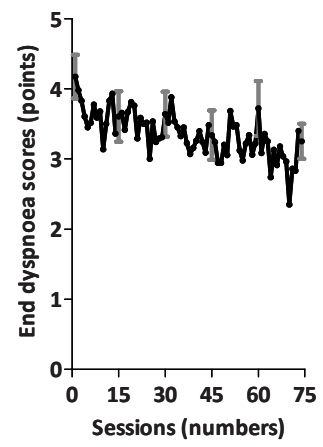

$\mathrm{F}$

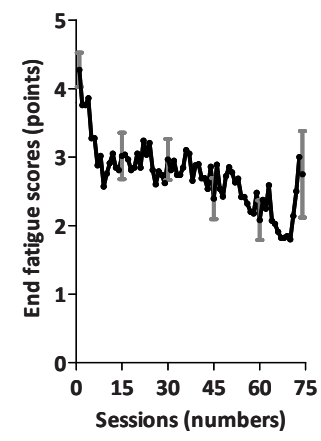

I

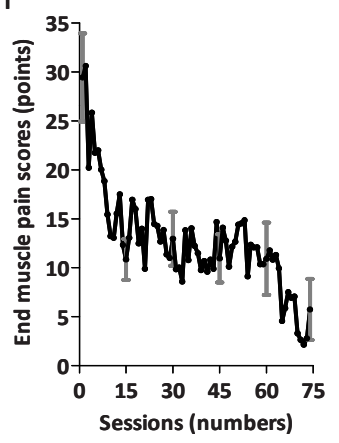




\section{REFERENCES}

1. Spruit MA, Vanderhoven-Augustin I, Janssen PP, et al. Integration of pulmonary rehabilitation in COPD. Lancet. 2008;371(9606):12-3.

2. Rabe KF, Hurd S, Anzueto A, et al. Global strategy for the diagnosis, management, and prevention of chronic obstructive pulmonary disease: GOLD executive summary. Am J Respir Crit Care Med. 2007;176(6):532-55.

3. Mahler DA, Wells CK. Evaluation of clinical methods for rating dyspnea. Chest. 1988;93(3):580-6.

4. Borges $\mathrm{O}$. Isometric and isokinetic knee extension and flexion torque in men and women aged 20-70. Scand J Rehabil Med. 1989;21(1):45-53.

5. Moher D, Hopewell S, Schulz KF, et al. CONSORT 2010 explanation and elaboration: updated guidelines for reporting parallel group randomised trials. BMJ. 2010;340:c869.

6. Wegmann H. The "new" Declaration of Helsinki. Jouirnal of International Biotechnology Law. 2009;6(4):173-6.

7. Sillen MJ, Franssen FM, Delbressine JM, et al. Heterogeneity in clinical characteristics and comorbidities in dyspneic individuals with COPD GOLD D: findings of the DICES trial. Respir Med. 2013;107(8):1186-94.

8. Spruit MA, Singh SJ, Garvey C, et al. An official american thoracic society/european respiratory society statement: key concepts and advances in pulmonary rehabilitation. Am J Respir Crit Care Med. 2013;188(8):e13-64.

9. Revill SI, Robinson JO, Rosen $\mathrm{M}$, et al. The reliability of a linear analogue for evaluating pain. Anaesthesia. 1976;31(9):1191-8.

10. Vivodtzev I, Lacasse Y, Maltais F. Neuromuscular electrical stimulation of the lower limbs in patients with chronic obstructive pulmonary disease. J Cardiopulm Rehabil Prev. 2008;28(2):79-91.

11. Vanderthommen M, Duchateau J. Electrical stimulation as a modality to improve performance of the neuromuscular system. Exerc Sport Sci Rev. 2007;35(4):180-5.

12. Sillen MJ, Wouters EF, Franssen FM, et al. Oxygen uptake, ventilation, and symptoms during lowfrequency versus high-frequency NMES in COPD: a pilot study. Lung. 2011;189(1):21-6.

13. Feigenbaum MS, Pollock ML. Strength training: rationale for current guidelines for adult fitness programs. The Physician and sportsmedicine. 1997;25(2):44-63.

14. Ratamess NA, Alvar BA, Evetoch TK, et al. Progression Models in Resistance Training for Healthy Adults. Medicine \& Science in Sports \& Exercise. 2009:687-708.

15. Spruit MA, Gosselink R, Troosters $T$, et al. Resistance versus endurance training in patients with COPD and peripheral muscle weakness. Eur Respir J. 2002;19(6):1072-8.

16. Mathur S, Makrides L, Hernandez P. Test-retest reliability of isometric and isokinetic torque in patients with chronic obstructive pulmonary disease. Physiother Can. 2004;56:94-101.

17. Franssen FM, Broekhuizen R, Janssen PP, et al. Limb muscle dysfunction in COPD: effects of muscle wasting and exercise training. Med Sci Sports Exerc. 2005;37(1):2-9.

18. Hernandes NA, Wouters EF, Meijer K, et al. Reproducibility of 6-minute walking test in patients with COPD. Eur Respir J. 2011;38(2):261-7.

19. Hul van 't A, Gosselink R, Kwakkel G. Constant-load cycle endurance performance: test-retest reliability and validity in patients with COPD. J Cardiopulm Rehabil. 2003;23(2):143-50.

20. Zigmond AS, Snaith RP. The hospital anxiety and depression scale. Acta Psychiatr Scand. 1983;67(6):361-70.

21. Jones PW, Quirk FH, Baveystock CM. The St George's Respiratory Questionnaire. Respir Med. 1991;85 Suppl B:25-31; discussion 3-7.

22. Annegarn J, Meijer K, Passos VL, et al. Problematic activities of daily life are weakly associated with clinical characteristics in COPD. J Am Med Dir Assoc. 2012;13(3):284-90.

23. Sewell L, Singh SJ. The Canadian Occupational Performance Measure: is it a reliable measure in clients with chronic obstructive pulmonary disease? Br J Occup Ther. 2001;64:305-10.

24. Graat-Verboom L, Smeenk FW, van den Borne BE, et al. Progression of osteoporosis in patients with COPD: a 3-year follow up study. Respir Med. 2012;106(6):861-70. 
25. O'Shea SD, Taylor NF, Paratz JD. A predominantly home-based progressive resistance exercise program increases knee extensor strength in the short-term in people with chronic obstructive pulmonary disease: a randomised controlled trial. Aust J Physiother. 2007;53(4):229-37.

26. Vestbo J, Prescott E, Almdal T, et al. Body mass, fat-free body mass, and prognosis in patients with chronic obstructive pulmonary disease from a random population sample: findings from the Copenhagen City Heart Study. Am J Respir Crit Care Med. 2006;173(1):79-83.

27. Graat-Verboom L, Wouters EF, Smeenk FW, et al. Current status of research on osteoporosis in COPD: a systematic review. Eur Respir J. 2009;34(1):209-18.

28. Janssen DJ, Spruit MA, Uszko-Lencer NH, et al. Symptoms, comorbidities, and health care in advanced chronic obstructive pulmonary disease or chronic heart failure. Journal of palliative medicine. 2011;14(6):735-43.

29. Diagnosis and classification of diabetes mellitus. Diabetes care. 2010;33 Suppl 1:S62-9.

30. Nutritional anaemias. Report of a WHO scientific group. World Health Organization technical report series. 1968;405:5-37. Epub 1968/01/01.

31. Alberti KG, Zimmet $P$, Shaw J. The metabolic syndrome--a new worldwide definition. Lancet. 2005;366(9491):1059-62.

32. Cockcroft DW, Gault MH. Prediction of creatinine clearance from serum creatinine. Nephron. 1976;16(1):31-41.

33. KDOQI Clinical Practice Guidelines and Clinical Practice Recommendations for Diabetes and Chronic Kidney Disease. American journal of kidney diseases : the official journal of the National Kidney Foundation. 2007;49(2 Suppl 2):S12-154.

34. 1999 World Health Organization-International Society of Hypertension Guidelines for the Management of Hypertension. Guidelines Subcommittee. Journal of hypertension. 1999;17(2):151-83.

35. Rautaharju PM, Warren JW, Jain U, et al. Cardiac infarction injury score: an electrocardiographic coding scheme for ischemic heart disease. Circulation. 1981;64(2):249-56. 



\section{CHAPTER 8}

Metabolic load during strength training or NMES in individuals with COPD: results from the DICES trial

Maurice JH Sillen, Frits ME Franssen, Anouk W Vaes, Jeannet ML Delbressine, Emiel FM Wouters, Martijn A Spruit

Submitted 


\section{ABSTRACT}

\section{Introduction}

Strength training and neuromuscular electrical stimulation (NMES) are effective training modalities for improving muscle function, exercise performance and health status in individuals with chronic obstructive pulmonary disease (COPD). The aim of the present study was to analyze the metabolic load of these training modalities at baseline, halfway, and at the end of an eight-week interdisciplinary pulmonary rehabilitation program in a subgroup of individuals with COPD participating in the DICES (Dyspneic Individuals with COPD: Electrical stimulation or Strength training) trial.

\section{Methods}

Of 24 individuals with COPD (FEV 1 : 34 $\pm 2 \%$ predicted, men: 58\%, age: 66 (61-68) years), intervention-related peak oxygen uptake $\left(\mathrm{VO}_{2}\right)$, peak minute ventilation (VE), transcutaneous oxygen saturation $\left(\mathrm{SpO}_{2}\right)$ and peak heart rate were assessed at three moments during their pulmonary rehabilitation program during a session of HF-NMES (75 Hertz), LF-NMES (15 Hertz) or strength training.

\section{Results}

Intervention-related peak $\mathrm{VO}_{2}$ did not change over time during HF-NMES, LF-NMES or strength training. Intervention-related peak VE did not change over time during strength training or LF-NMES and increased slightly, but significantly over time during HF-NMES. Peak $\mathrm{VO}_{2}$ and VE were significantly higher during strength training compared to HF-NMES or LF-NMES. Oxygen saturation significantly decreased after the first measurements during HF-NMES and strength training group to baseline, while no significant changes in oxygen saturation were observed during the other measurements. Heart rate significantly increased compared to baseline in all groups at all moments and was significantly higher after strength training compared to HF-NMES or LF-NMES. Median end scores (points) for dyspnea, fatigue and muscle pain ranged from 1 to 3, from 0.5 to 2 and from 0 to 6 after HF-NMES, from 2 to 3, from 2 to 5 and from 0 to 9 after LF-NMES and from 2 to 5 , from 1.5 to 4 and from 0 to 28 after strength training respectively.

\section{Conclusions}

To conclude, the metabolic load and symptom scores remain acceptable low over time with increasing training loads during HF-NMES, LF-NMES or strength training. 


\section{INTRODUCTION}

Individuals with chronic obstructive pulmonary disease (COPD) may suffer from lowerlimb muscle weakness and poor exercise capacity, in particular those with severe to very severe dyspnea (1-3). This is most probably due to reductions in weight-bearing daily physical activities (4). Therefore, an exercise-based pulmonary rehabilitation program may be beneficial (5). Severely dyspneic individuals with COPD (i.e., modified MRC dyspnea grade 3 or 4), however, are less likely to complete a pulmonary rehabilitation program (6). This may be due to exercise-induced dyspnea, particularly during whole-body endurance training (7). Therefore, strength training (8) or transcutaneous neuromuscular electrical stimulation (NMES) $(9,10)$ may be preferential alternative rehabilitative modalities for severely dyspneic individuals with COPD $(11,12)$. These interventions are safe and effective in severely dyspneic individuals with COPD and quadriceps muscle weakness at baseline (13). Indeed, lower-limb muscle function, functional exercise performance, problematic activities of daily life, mood status, and health status improved significantly following eight weeks of strength training, high-frequency (HF, 75 Hertz) NMES, or low-frequency (LF, 15 Hertz) NMES (13).

A major advantage of strength training and NMES is the relatively low metabolic load (e.g., the intervention-related peak oxygen uptake $\left(\mathrm{VO}_{2}\right)$ and ventilation (VE)), accompanied with relatively low dyspnea symptom scores $(14,15)$. The metabolic load during multiple successive sessions of strength training has been reported once in 11 individuals with COPD (7). The leg press strengthening exercise increased significantly during a 12-week pulmonary rehabilitation program ( $+43 \%$ of baseline training load), accompanied by a significant increase in metabolic load over time $(+23 \%$ of baseline intervention-related peak $\mathrm{VO}_{2} ;+18 \%$ of baseline intervention-related peak VE) (7). The metabolic load during a session of high-frequency (HF) or low-frequency (LF) NMES has only been measured cross-sectionally $(14,15)$. Whether and to what extent the metabolic load will remain stable over time while the NMES pulse amplitude is expected to increase (16) remains currently unknown in individuals with COPD. Moreover, the actual course of NMES pulse amplitude has never been described in individuals with COPD. This, however, will provide a better insight in the feasibility and efficacy of these types of local muscle training.

The aim of the present study was to analyze the metabolic load of the different local muscle training modalities at baseline, half-way, and at the end of the eight-week program in a subgroup of individuals with COPD who participated in the DICES (Dyspneic Individuals with COPD: Electrical stimulation or Strength training) trial. A priori, we hypothesized that individuals with COPD are able to increase the strength 
training load or NMES pulse amplitude (irrespective of stimulation frequency), while the metabolic load will remain stable compared to baseline.

\section{METHODS}

\section{Participants}

In the DICES trial, individuals with COPD with MMRC dyspnea grade 3 or 4 (2), and quadriceps weakness (17) were randomly assigned to lower-limb HF-NMES (75 Hertz), lower-limb LF-NMES (15 Hertz), or lower-limb strength training (leg extension strengthening exercise, and leg press strengthening exercise $(18,19))$. These interventions took place in group sessions, twice per day, 5 times per week for 8 weeks. All sessions were supervised by a physiotherapist. The interdisciplinary treatment was identical for all participants, and treadmill walking or stationary ergometry cycling was not applied during the trial. Symptom scores for dyspnea, fatigue, and muscle pain were assessed before and after each session (20).

The Medical Ethical Committee of the Maastricht University Medical Centre+ (MEC 093-072) approved this trial, which conformed to the principles outlined in the World Medical Association declaration of Helsinki which was revised in Seoul (21). Details of the trial were registered at http://www.trialregister.nl (NTR2322) before first subject enrolment. All patients gave written informed consent to participate in the study and a subgroup additionally gave written informed consent to undergo the measurements of the metabolic load. Some of the baseline findings, and the efficacy data of the DICES trial have been published before $(13,22)$.

\section{NMES PROTOCOLS}

Quadriceps muscles and calf muscles of both legs were stimulated electrically with a portable battery-operated electrical stimulator (Tensmed S84, Enraf-Nonius, Rotterdam, the Netherlands). The output characteristics of the device have been checked on an oscilloscope. A total of eight carbon-rubber electrodes in moistened sponges were placed on the target muscles (four electrodes on each leg): two pairs of $8 \times 12 \mathrm{~cm}$ on the quadriceps muscles and two pairs of $4 \times 6 \mathrm{~cm}$ on the calf muscles. The electrodes on the quadriceps muscles were placed transversally $5-10 \mathrm{~cm}$ distal to the inguinal fold and $4-8 \mathrm{~cm}$ proximal to the patella. The electrodes on the calf muscles were placed longitudinally on the belly of the gastrocnemeii muscles. Both NMES protocols used a symmetrical biphasic square pulse with pulse duration of $400 \mu \mathrm{s}$. The contraction time was 6 seconds with 8 seconds relaxation excluding 1 second ramp-up and 1 second ramp-down. Thus, the total cycle length was 16 seconds. After a continuous warm-up of 3 minutes at 5 Hertz, pulse amplitude was adjusted to 
individual toleration during each session lasting 18 minutes. The frequencies used were 75 Hertz (HF-NMES) or 15 Hertz (LF-NMES) (15). Participants were blinded for the NMES frequency.

\section{MusCle StRENGTH TRAINING}

Muscle strength training consisted of bilateral leg extension and bilateral leg press exercises (Technogym SpA, Gambettola, Italy) $(18,19)$. The one-repetition maximum (1RM) was determined during the initial assessment to target the training load. 1RM was defined as that load which could be lifted just one time. Both exercises started at $70 \%$ of 1RM, 4 sets of 8 repetitions per exercise with at least 2 minutes of recovery between each set. The training load was set to increase with $5 \%$ each two weeks (19).

\section{OUtCOMES}

Outcome assessors were blinded for treatment allocation. The investigators supervising the interventions (MJHS, AWV) were blinded for the initial results, and were not involved in the outcome assessment. Participants were instructed to not divulge their group allocation. Participants, who were randomly assigned to one of the NMES groups, were blinded for the type of stimulation frequency.

\section{QUADRICEPS MUSCLE FUNCTION}

Quadriceps muscle function (i.e., peak muscle strength and muscle endurance), using a Biodex (Biodex System 4 Pro, Biodex Medical Systems, Inc., New York, USA). Quadriceps peak muscle strength (Newton-meter, $\mathrm{Nm}$ ) and quadriceps muscle endurance (Joules, J) were measured isokinetically. The participants performed thirty volitional maximal contractions at an angular velocity of $90^{\circ}$ per second.

\section{FUNCTIONAL EXERCISE PERFORMANCE}

Functional exercise performance was measured with the 6-min walk test, including a practice walk at initial assessment (23). The best 6-min walk distance (6MWD) was used for further analyses. The constant work-rate cycling endurance test (CWRT, expressed in seconds) was performed at $75 \%$ of the pre-determined peak cycling rate, which has a high reliability in individuals with COPD (24). Symptom scores for exercise-induced dyspnea and fatigue were assessed before and after these exercise tests.

\section{Metabolic load}

During both NMES sessions continuous on-line calculations of breath-by-breath oxygen uptake $\left(\mathrm{VO}_{2}\right)$ and minute ventilation (VE) were obtained using the Oxycon mobile, a portable metabolic system (Carefusion the Netherlands, Houten, the Netherlands). 
After calibration the face mask (Combitox, Dräger Safety, Lübeck, Germany) was carefully adjusted to the patient's face and checked for air leaks. Data were processed in the PC-software (JLAB version 5.20b, Carefusion the Netherlands, Houten, the Netherlands) and were collected breath by breath. The metabolic load was measured during sessions of HF-NMES, LF-NMES, or strength training in the first week, the fourth week, and in the last week of the trial. This methodology has been used before in individuals with COPD $(14,15)$.

\section{StATistical ANALYSIS}

Analyses were performed using SPSS for Windows, Version 17.0.1 (SPSS, Inc., Chicago, II, USA). Descriptive statistics were presented as median and interquartile range unless otherwise stated. Differences within groups were analyzed using Wilcoxon signed rank test. Groups were compared using Mann-Whitney $U$ test or Kruskal-Wallis one-way analysis of variance. All tests were two-sided using a significance level of $5 \%$.

\section{RESULTS}

\section{BASELINE CHARACTERISTICS}

Of the 120 individuals who participated in the DICES trial, 61 individuals (51\%) were ineligible for the additional metabolic data collection due to the use of long-term oxygen therapy. Moreover, 26 individuals (22\%) did not consent to this extra set of tests and 3 individuals (2\%) who gave informed consent withdrew before start. At baseline, from 30 individuals (25\%) intervention-related peak $\mathrm{VO}_{2}, \mathrm{VE}$, heart rate and oxygen saturation were obtained. From 24 individuals the metabolic load was measured during a session of HF-NMES $(n=9)$, LF-NMES $(n=7)$, or strength training $(n=8)$ in the first week, the fourth week, and in the last week of the trial (Figure 8.1).

The 24 individuals of this pre-specified sub-analysis had severe to very severe COPD, a poor diffusing capacity for carbon monoxide and a poor functional and peak exercise performance (Table 8.1). Besides age and the relative peak ventilation during the cardiopulmonary exercise test, baseline characteristics did not significantly differ between intervention groups (Table 8.1$)$. Mean FEV $(34 \pm 2 \%$ versus $31 \pm 1 \%$ pred), $\mathrm{PaCO}_{2}(5.2 \pm 0.1 \mathrm{kPa}$ versus $5.8 \pm 0.1 \mathrm{kPa})$, fat-free mass index $\left(17.2 \pm 0.4 \mathrm{~kg} / \mathrm{m}^{2}\right.$ versus $\left.16.3 \pm 0.2 \mathrm{~kg} / \mathrm{m}^{2}\right)$ and total work $(1389 \pm 95 \mathrm{~J}$ versus $1122 \pm 49 \mathrm{~J})$ were significantly higher in the metabolic load group compared to the remaining group, other baseline characteristics (age, DLco, residual volume, $\mathrm{PaO}_{2}, \mathrm{SO}_{2}$, peak and functional exercise performance, body mass index, peak torque, HADS anxiety and depression and health status) were not significantly different between both groups (Supplemental Table S8.1). 
Figure 8.1 Flow diagram of measurements metabolic load.

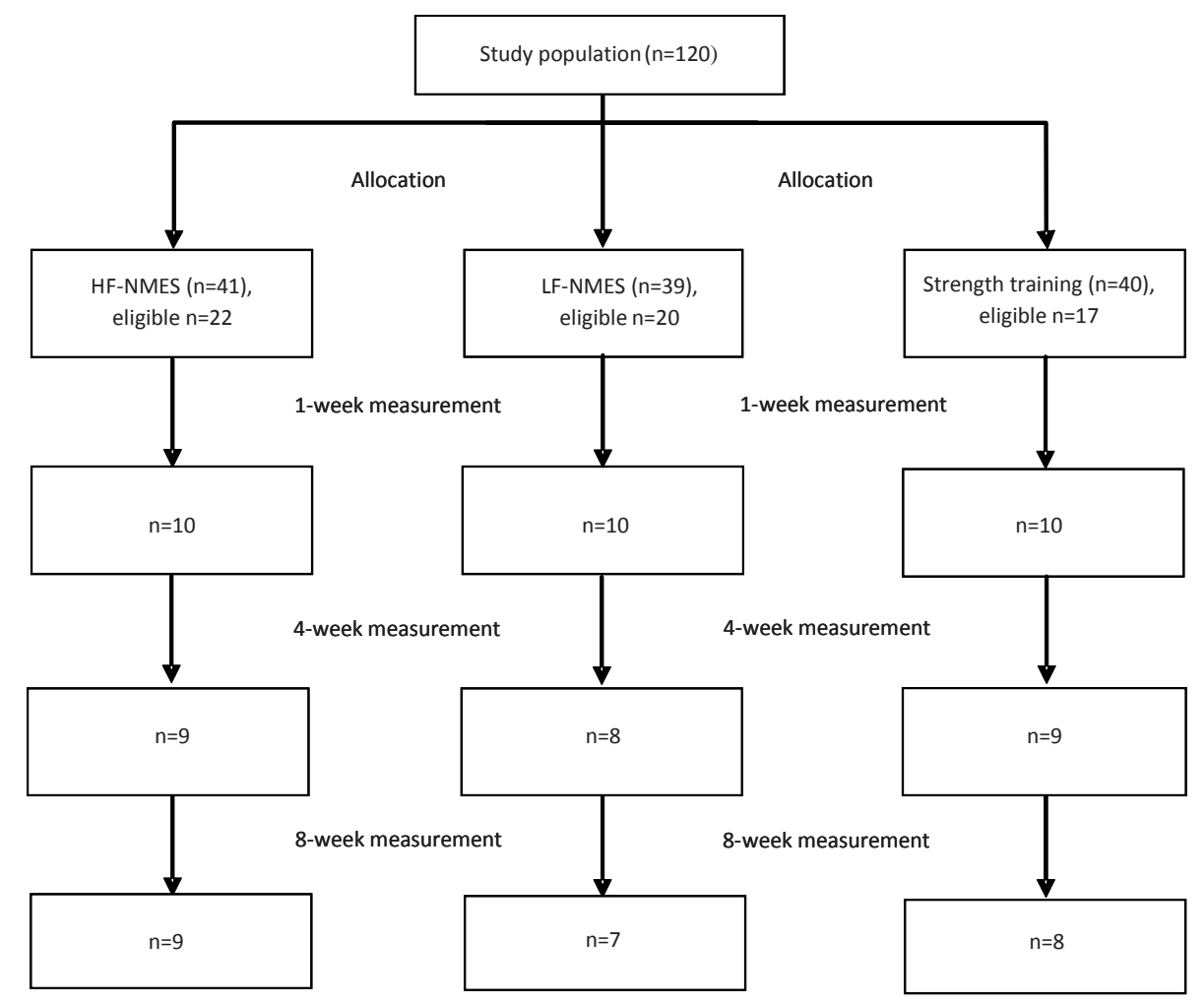

\section{EFFICACY OF INTERVENTIONS}

\section{Quadriceps muscle strength}

Isokinetic quadriceps peak torque increased significantly following HF-NMES (12.6 Nm (3.0-17.5 Nm); $p=0.021)$, but not following LF-NMES (4.2 Nm (-5.2-5 Nm); $p=0.866)$ or strength training $(5.7 \mathrm{Nm}(-9-22 \mathrm{Nm}) ; \mathrm{p}=0.263)$ (Figure 8.2). There were no significant between-group differences in changes.

\section{Quadriceps muscle endurance}

Isokinetic total work increased significantly following HF-NMES (292 J (156-501 J); $\mathrm{p}<0.01)$, but not following LF-NMES (12 J $(-73-178 \mathrm{~J}) ; \mathrm{p}=0.499)$ or strength training (157 J (-185-456 J); $p=0.263$ ) (Figure 8.2). The improvement following HF-NMES was significantly higher compared to LF-NMES ( $p=0.005)$ (Figure 8.2). 
Table 8.1 General characteristics.

\begin{tabular}{|c|c|c|c|c|c|}
\hline & $\begin{array}{c}\text { Total group } \\
n=24\end{array}$ & $\begin{array}{c}\text { HF-NMES } \\
n=9\end{array}$ & $\begin{array}{c}\text { LF-NMES } \\
n=7\end{array}$ & $\begin{array}{l}\text { Strength training } \\
\mathrm{n}=8\end{array}$ & P-value \\
\hline $\operatorname{Sex}(M / F)$ & $14 / 10$ & $6 / 3$ & $4 / 173$ & $4 / 4$ & 0.791 \\
\hline Age (years) & $66(61-68)$ & $64(59-67)$ & $62(59-67)$ & $71(66-77)$ & 0.012 \\
\hline \multicolumn{6}{|l|}{ Pulmonary function } \\
\hline $\mathrm{FEV}_{1}$ (liters) & $0.98(0.78-1.05)$ & $1.01(0.84-1.19)$ & $0.74(0.60-0.84)$ & $1.00(0.96-1.10)$ & 0.010 \\
\hline $\mathrm{FEV}_{1}(\%$ predicted) & $35(29-51)$ & $34(23-51)$ & $30(20-38)$ & $46(43-60)$ & 0.064 \\
\hline $\mathrm{FEV}_{1} / \mathrm{VC} \max (\%)$ & $32(24-43)$ & $31(24-41)$ & $25(24-29)$ & $42(33-52)$ & 0.057 \\
\hline $\mathrm{DL}_{\mathrm{CO}}(\%)$ & $42(36-55)$ & $41(31-55)$ & $42(34-61)$ & $46(40-56)$ & 0.802 \\
\hline RV (\%) & $177(136-235)$ & $190(137-243)$ & $208(172-260)$ & $146(128-178)$ & 0.081 \\
\hline \multicolumn{6}{|l|}{ Arterial blood gases } \\
\hline $\mathrm{PaO}_{2}(\mathrm{kPa})$ & $9.8(8.8-10.8)$ & $9.0(8.5-10.9)$ & $9.6(8.5-9.9)$ & $10.3(9.4-11.2)$ & 0.284 \\
\hline $\mathrm{PaCO}_{2}(\mathrm{kPa})$ & $4.9(4.7-5.5)$ & $5.4(4.8-5.7)$ & $5.0(4.7-5.6)$ & $4.8(4.6-5.1)$ & 0.159 \\
\hline $\mathrm{SpO}_{2}(\%)$ & $96(95-97)$ & 95 (95-97) & 95 (94-97) & $97(96-98)$ & 0.166 \\
\hline $\begin{array}{l}\text { GOLD classification } \\
(\mathrm{I} / \mathrm{II} / \mathrm{III} / \mathrm{IV})\end{array}$ & $0 / 7 / 10 / 7$ & $0 / 3 / 3 / 3$ & $0 / 0 / 4 / 3$ & $0 / 4 / 3 / 1$ & 0.025 \\
\hline $\begin{array}{l}\text { GOLD classification } \\
\text { (new) }\end{array}$ & $0 / 3 / 0 / 21$ & $0 / 2 / 0 / 7$ & $0 / 0 / 0 / 7$ & $0 / 1 / 0 / 7$ & 0.427 \\
\hline \multicolumn{6}{|l|}{$(A / B / C / D)$} \\
\hline BMI $\left(\mathrm{kg} / \mathrm{m}^{2}\right)$ & $25.4(22.2-29.8)$ & 25.4 (21.9-26.9) & $24.6(23.5-30.3)$ & $26.9(21.5-31.1)$ & 0.834 \\
\hline FFMI $\left(\mathrm{kg} / \mathrm{m}^{2}\right)$ & $17.0(15.8-18.5)$ & $16.9(15.7-17.5)$ & 16.5 (15.7-18.8) & $17.4(15.8-19.1)$ & 0.680 \\
\hline \multicolumn{6}{|c|}{ Cardiopulmonary exercise test } \\
\hline Peak load (watts) & $47(35-57)$ & $51(35-57)$ & $46(41-62)$ & $44(30-58)$ & 0.867 \\
\hline Peak load (\% predicted) & $33(23-47)$ & $26(23-39)$ & $38(22-81)$ & $39(23-63)$ & 0.481 \\
\hline Peak $\mathrm{VO}_{2}(\mathrm{ml} / \mathrm{min})$ & $841(710-987)$ & 751 (697-919) & 817 (734-1087) & 904 (699-1076) & 0.473 \\
\hline Peak $\mathrm{VO}_{2}$ (\% predicted) & $48(33-73)$ & $31(27-64)$ & $53(40-90)$ & $52(40-115)$ & 0.142 \\
\hline Peak VE (liters) & $33(29-39)$ & $38(28-41)$ & $32(25-35)$ & $35(29-44)$ & 0.553 \\
\hline Peak VE (\% MVV) & $89(78-107)$ & $84(72-98)$ & $103(95-119)$ & $81(68-101)$ & 0.040 \\
\hline Peak HR (bpm) & $104(98-124)$ & $103(98-122)$ & $126(104-131)$ & $102(90-109)$ & 0.080 \\
\hline Peak HR (\% predicted) & $71(64-75)$ & $66(64-73)$ & $75(68-83)$ & $70(63-72)$ & 0.186 \\
\hline Dyspnea, end (points) & $7(5-8)$ & $7(5-7.5)$ & $7(7-9)$ & $8(5-9.5)$ & 0.526 \\
\hline Fatigue, end (points) & $5(3-7)$ & $5(3.5-7.5)$ & $5(3-7)$ & $4(3-7)$ & 0.861 \\
\hline $\mathrm{SpO}_{2}$, end (\%) & $91(89-95)$ & $93(91-98)$ & $90(89-91)$ & $94(89-96)$ & 0.132 \\
\hline
\end{tabular}

Values expressed as median (interquartile range) or numbers. HF-NMES=High-frequency transcutaneous neuromuscular electrical stimulation; LF-NMES=Low-frequency transcutaneous neuromuscular electrical stimulation; $\mathrm{M}=$ males; $\mathrm{F}=$ females; $\mathrm{FEV}_{1}=$ forced expiratory volume in one second; $\mathrm{VC}$ max=maximum vital capacity; $\mathrm{DL}_{\mathrm{CO}}=$ diffusion capacity of the lung for carbon monoxide; $\mathrm{RV}=$ residual volume; $\mathrm{PaO}_{2}=$ resting arterial oxygen tension; $\mathrm{PaCO}_{2}=$ resting arterial carbon dioxide tension; $\mathrm{kPa}=$ kilopascal; $\mathrm{LTOT}=$ long-term oxygen therapy; GOLD=Global Initiative on Obstructive Lung Diseases; BMI=body mass index; FFMI=fat free mass index; $\mathrm{kg} / \mathrm{m}^{2}=$ kilogram per square meter; $\mathrm{VO}_{2}=$ oxygen uptake; $\mathrm{SpO}_{2}=$ transcutaneous oxygen saturation; $\mathrm{ml} / \mathrm{min}=$ milliliters per minute; $\% \mathrm{MVV}=$ percentage maximal voluntary ventilation; bpm=beats per minute. 
Figure 8.2 Individual changes in quadriceps muscle strength and endurance from baseline to end.

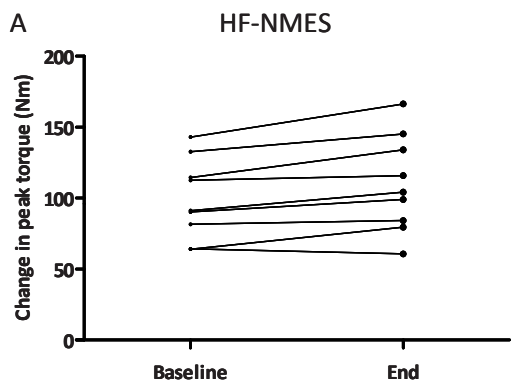

$\mathrm{C}$

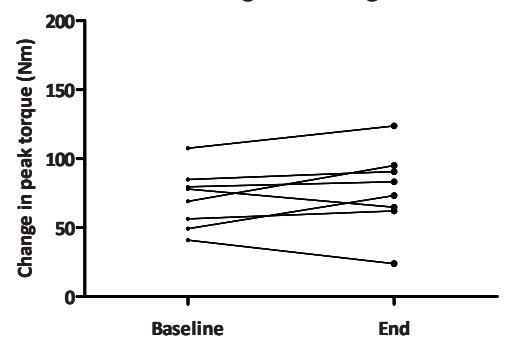

E

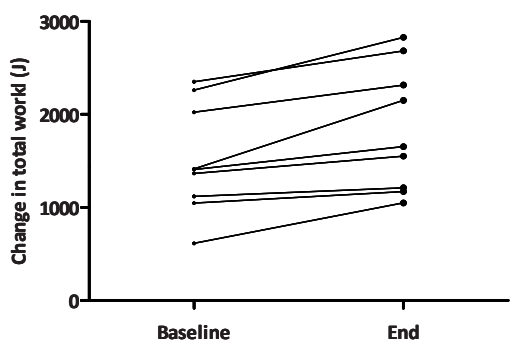

g

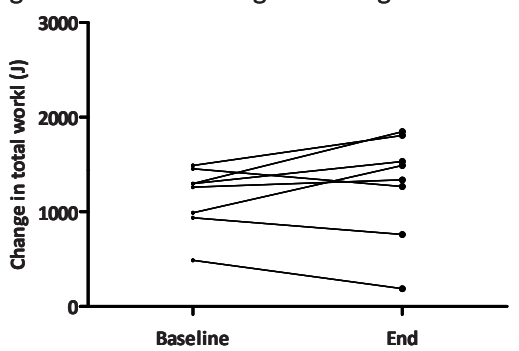

B LF-NMES

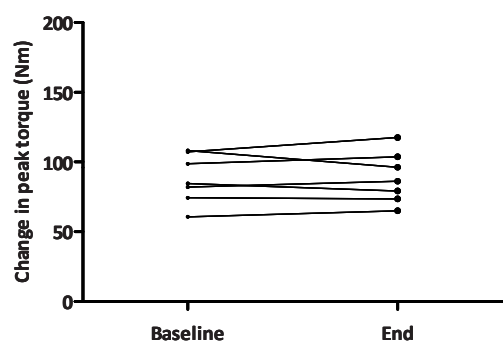

$\mathrm{F}$

LF-NMES

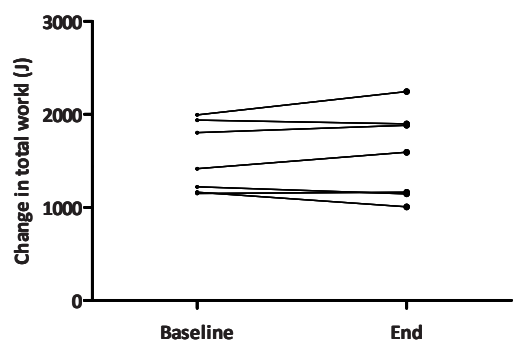

HF-NMES=high-frequency transcutaneous neuromuscular electrical stimulation; LF-NMES=low-frequency transcutaneous neuromuscular electrical stimulation (Changes in quadriceps peak torque: $2 \mathrm{~A}, 2 \mathrm{~B}$ and $2 \mathrm{C}$; changes in quadriceps muscle endurance: $2 \mathrm{E}, 2 \mathrm{~F}$ and $2 \mathrm{G}$ ). 


\section{Six-minute walk distance}

6MWD improved significantly following HF-NMES (75 m (29-138 m); $p=0.008)$ or strength training $(57 \mathrm{~m}(32-85 \mathrm{~m}) ; \mathrm{p}=0.034)$, but not following LF-NMES $(27 \mathrm{~m}$ $(-34-65 \mathrm{~m}) ; p=0.310)$. There were no significant differences in changes between groups.

\section{Constant work-rate test}

Endurance time during the constant work-rate cycling test improved significantly following HF-NMES (92 s (49-275 s); $p=0.066)$, LF-NMES (83 s (-7-223 s); $p=0.091$ ) or strength training (37 s (1-98 s); $p=0.043)$. There were no significant differences in changes between groups.

\section{COURSE OF HF-NMES, LF-NMES OR STRENGTH TRAINING}

In the HF-NMES group, pulse amplitude ranged from 12 to $40 \mathrm{~mA}$ in week one to 34 to $71 \mathrm{~mA}$ in week eight ( $p=0.008$ ); and in the LF-NMES group from 25 to $50 \mathrm{~mA}$ to 35 to 98 $\mathrm{mA}(\mathrm{p}=0.063$, Figure 8.3). Median end dyspnea scores, end fatigue scores and end muscle pain scores ranged from 1 to 3 points, from 0.5 to 2 points and from 0 to 6 points in the HF-NMES group and from 2 to 3 points, from 2 to 5 points and from 0 to 9 points in the LF-NMES group respectively. The load during leg extension strengthening exercise ranged from 2.5 to $20 \mathrm{~kg}$ in week one to 12.5 to $27.5 \mathrm{~kg}$ in week eight; and from 5 to $70 \mathrm{~kg}$ to 35 to $90 \mathrm{~kg}$ for the leg press strengthening exercise (both $\mathrm{p}<0.02$, Figure 8.3). In addition, median end dyspnea scores, end fatigue scores and end muscle pain scores ranged from 2 to 5 points, from 1.5 to 4 points and from 0 to 28 points in the strength training group.

\section{Metabolic load dURING HF-NMES, LF-NMES OR STRENGTH TRAINING}

Intervention-related peak $\mathrm{VO}_{2}$ did not change over time all three interventions (Figure 8.4A). Intervention-related peak VE did not change over time in the strength training of LF-NMES group ( $p>0.171)$. In the HF-NMES group, the intervention-related VE increased slightly, but significantly over time $(\mathrm{p}=0.012$, Figure $8.4 \mathrm{~B})$. At all measurement points, intervention-related peak $\mathrm{VO}_{2}$ and $\mathrm{VE}$ were significantly higher during strength training sessions compared to the HF-NMES or LF-NMES sessions (all $p<0.05$ ). There were no differences between HF-NMES and LF-NMES ( $p>0.28$ ). Oxygen saturation (lowest values) was significant lower at the end of the fist measurements in the HF-NMES group and the strength training group compared with baseline (both $p<0.03$ ), during the other measurements no significant changes in oxygen saturation were observed (all $p>0.05$ ) (Table 8.2). There were no significant between-group differences in changes in oxygen saturation (all $p>0.05$ ). Heart rate was significantly higher at the end compared to baseline in all groups in all measurements $(p<0.05)$ (Table 8.2).During all 
measurements, changes in heart rate were significantly higher after strength training compared to HF-NMES or LF-NMES $(p<0.04)$.

Figure 8.3 Course in pulse amplitude and training load during the rehabilitation program.

A

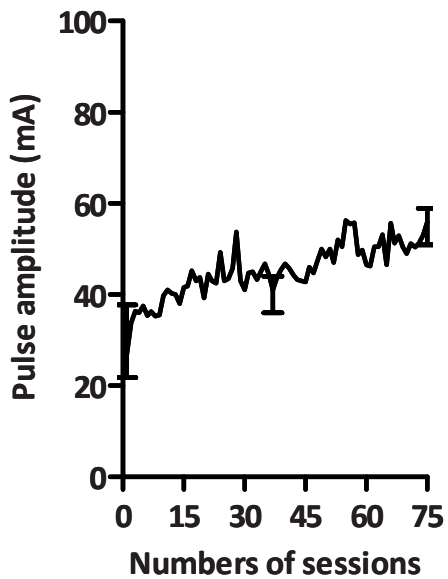

C

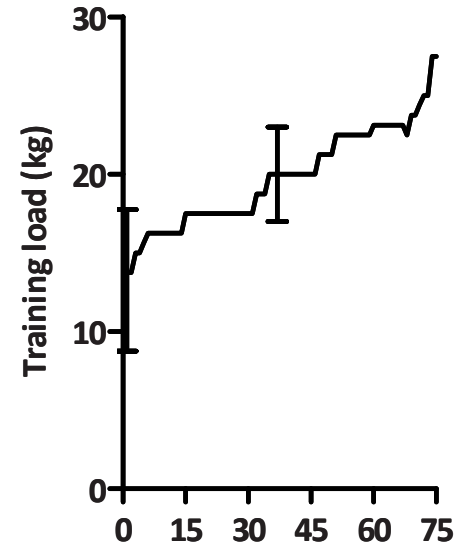

Numbers of sessions
B LF-NMES

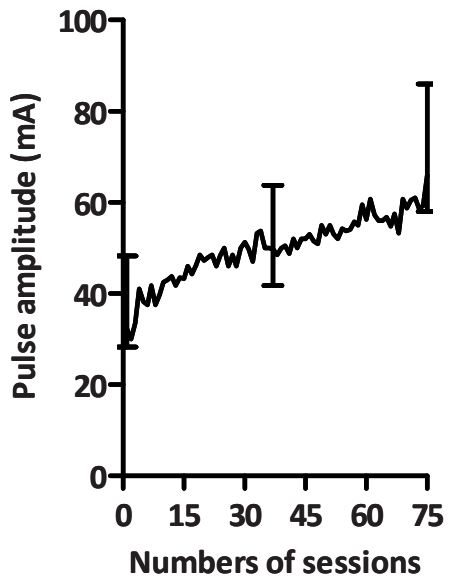

D

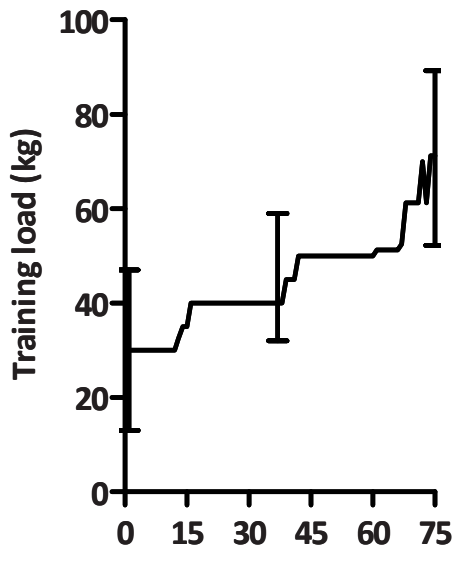

Numbers of sessions

HF-NMES=high-frequency transcutaneous neuromuscular electrical stimulation; LF-NMES=low-frequency transcutaneous neuromuscular electrical stimulation. 
Figure 8.4 Intervention-related $\mathrm{VO}_{2}$ and VE during HF-NMES, LF-NMES or strength training.

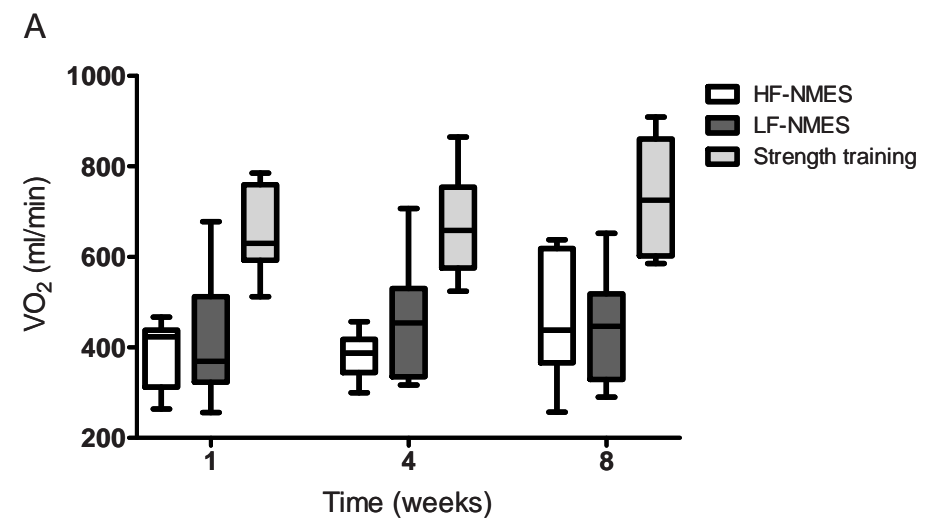

B

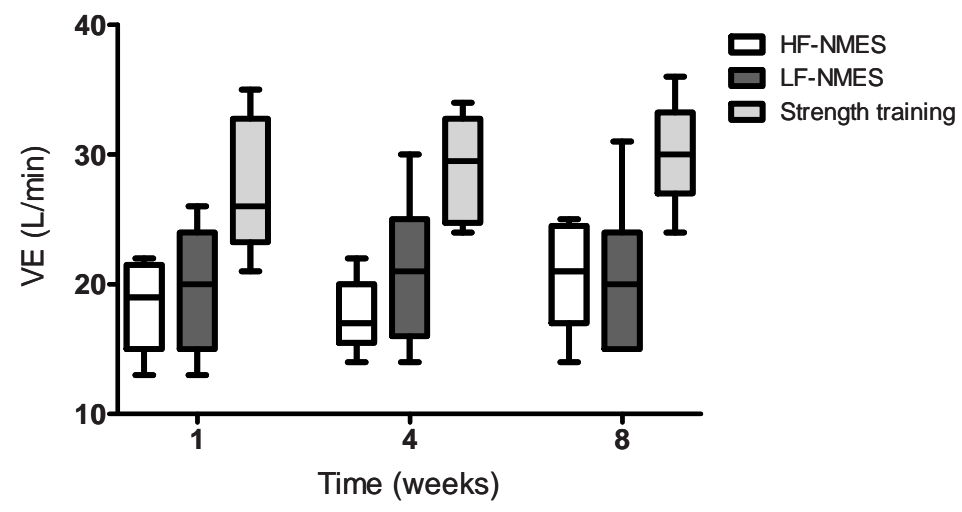

HF-NMES=high-frequency transcutaneous neuromuscular electrical stimulation; LF-NMES=low-frequency transcutaneous neuromuscular electrical stimulation; $\mathrm{VO}_{2}=$ oxygen uptake; $\mathrm{V}_{\mathrm{E}}=$ ventilation. 


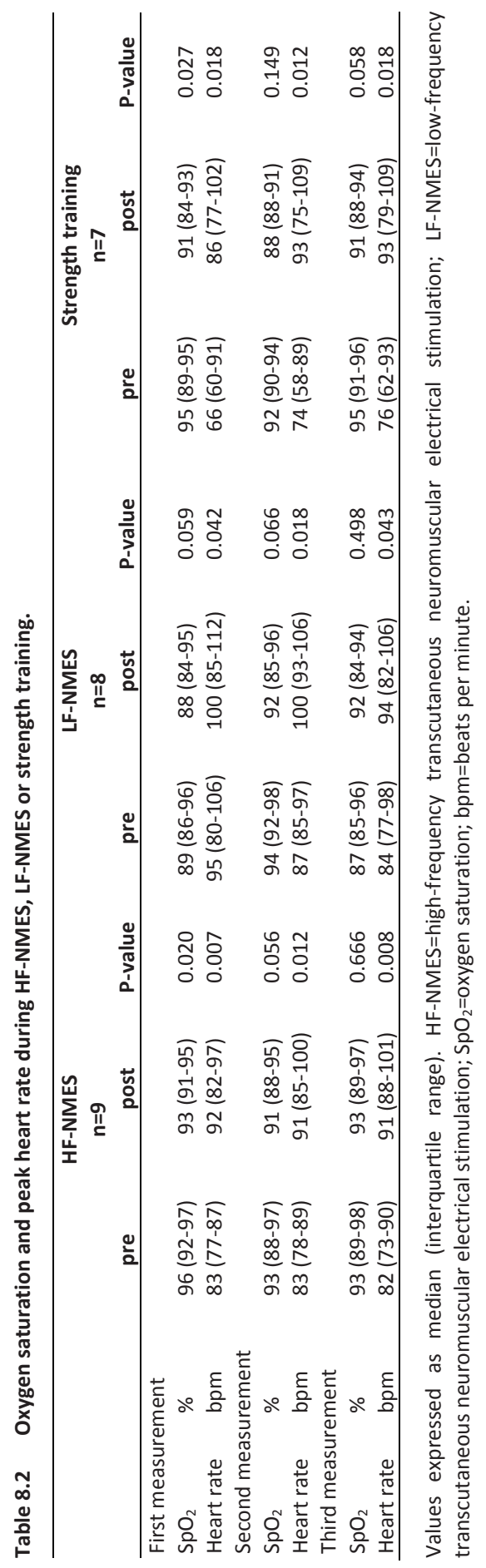




\section{DISCUSSION}

This is the first study to investigate the metabolic load of various local muscle training modalities in severely dyspneic COPD patients with quadriceps muscle weakness during the course of an inpatient pulmonary rehabilitation program. It showed that the metabolic load measured during successive sessions of strength training, HF-NMES, or LF-NMES remained generally stable, while the NMES pulse amplitude or the strength training load increased significantly during the eight-week intervention period.

The median oxygen uptake during the baseline session of HF-NMES, LF-NMES or strength training ranged between 30 and $99 \%$ of the peak aerobic capacity measured during the cardiopulmonary exercise test, and was comparable with previous studies $(7,14,15)$. At all measurement points, intervention-related peak $\mathrm{VO}_{2}$ and $\mathrm{VE}$ were significantly lower during NMES (LF and HF) compared with strength training. This is in line with previous studies measuring the metabolic load in patients with COPD during NMES or strength training $(14,15)$. However, these results are in contrast with the findings of a study in healthy male recreational athletes comparing one session of voluntary contractions with one session of HF-NMES $(75 \mathrm{~Hz})$ (25). Theurel and colleagues found that the average oxygen consumption and ventilation were significantly higher during HF-NMES compared with voluntary exercise (25). Besides the study design and subjects, an important difference with the present study is the training load (25). Theurel and colleagues used an average training load in strength training of $46 \%$ of the maximal voluntary contraction instead of $60-70 \%$ of the onerepetition maximum which is used in our study and what is recommended by the American College of Sports Medicine (8).

The present study shows no changes over time in the metabolic load which is not in line with the study of Probst and colleagues (7). Probst and colleagues showed a significant increase in oxygen uptake and ventilation during a $12 \mathrm{wk}$ program of leg press exercises (7). This could be attributable to a greater increase in the training load. However, the change in training load of leg press exercises was comparable to the present study.

The median increase in pulse amplitude during the study was $24 \mathrm{~mA}$ in HF-NMES and $37 \mathrm{~mA}$ in LF-NMES. The course in pulse amplitude is comparable with previous studies in severely disabled patients with COPD which respond to $\operatorname{NMES}(16,26)$. The low metabolic load accompanied with acceptable low dyspnea and fatigue scores probably explains the applicability of these interventions in severely disabled and dyspneic patients, even during acute COPD exacerbations (26-28). This is also probably the reason that NMES can easily be applied in bed-bound individuals with chronic hypercapnic respiratory failure due to COPD who are receiving mechanical ventilation (29) or critically ill patients in the intensive care unit (30).

Because of the constant metabolic load in combination with stable symptom scores over time, it seems reasonable to hypothesize that the improvements in muscle 
function are at least partially due to intramuscular changes. Previously, it has been shown that type I and Ila fibers increased following LF-NMES $(31,32)$ or HF-NMES $(33,34)$. Strength training generally results in increased levels of glycolytic enzymes (35) and an increase in percentage and size of type II fibers (36-39).

Obviously, this study has some limitations. First, the inclusion criteria of the DICES trial limits the external validity of the present findings. Only COPD patients with an mMRC score of 3 and 4 in combination with muscle weakness were included. Secondly, the small sample size and selected patient characteristics for participation in the measurements with the Oxycon mobile may be an important reason for detecting no significant improvements in peak muscle strength in the strength training group and quadriceps muscle endurance in the LF-NMES group and the strength training group. Moreover, this small sample size may limit the external validity and broad applicability of these findings. Only patients without long-term oxygen therapy were eligible to participate due to the methodology used (7).

To conclude, the metabolic load and symptom scores for dyspnea, fatigue and muscle pain remain acceptable low over time with increasing training loads during HF-NMES, LF-NMES or strength training. For this reason, these interventions are recommended in severely dyspneic patients with COPD for improving their muscle function and exercise performance. 


\section{REFERENCES}

1. Seymour JM, Spruit MA, Hopkinson NS, Natanek SA, Man WD, Jackson A, et al. The prevalence of quadriceps weakness in COPD and the relationship with disease severity. Eur Respir J. 2010;36(1):81-8.

2. Spruit MA, Pennings HJ, Janssen PP, Does JD, Scroyen S, Akkermans MA, et al. Extra-pulmonary features in COPD patients entering rehabilitation after stratification for MRC dyspnea grade. Respiratory medicine. 2007;101(12):2454-63.

3. Spruit MA, Watkins ML, Edwards LD, Vestbo J, Calverley PM, Pinto-Plata V, et al. Determinants of poor 6-min walking distance in patients with COPD: the ECLIPSE cohort. Respir Med. 2010;104(6):849-57.

4. Waschki B, Spruit MA, Watz H, Albert PS, Shrikrishna D, Groenen M, et al. Physical activity monitoring in COPD: compliance and associations with clinical characteristics in a multicenter study. Respir Med. 2012;106(4):522-30.

5. Spruit MA, Singh SJ, Garvey C, Zuwallack R, Nici L, Rochester C, et al. An official american thoracic society/european respiratory society statement: key concepts and advances in pulmonary rehabilitation. Am J Respir Crit Care Med. 2013;188(8):e13-64.

6. Hogg L, Garrod R, Thornton H, McDonnell L, Bellas H, White P. Effectiveness, attendance, and completion of an integrated, system-wide pulmonary rehabilitation service for COPD: prospective observational study. Copd. 2012;9(5):546-54.

7. Probst VS, Troosters T, Pitta F, Decramer M, Gosselink R. Cardiopulmonary stress during exercise training in patients with COPD. Eur Respir J. 2006;27(6):1110-8.

8. American College of Sports Medicine position stand. Progression models in resistance training for healthy adults. Med Sci Sports Exerc. 2009;41(3):687-708.

9. Maffiuletti NA. Physiological and methodological considerations for the use of neuromuscular electrical stimulation. Eur J Appl Physiol. 2010;110(2):223-34

10. Vanderthommen M, Duchateau J. Electrical stimulation as a modality to improve performance of the neuromuscular system. Exerc Sport Sci Rev. 2007;35(4):180-5.

11. Spruit MA, Wouters EF. New modalities of pulmonary rehabilitation in patients with chronic obstructive pulmonary disease. Sports Med. 2007;37(6):501-18.

12. Sillen MJ, Speksnijder CM, Eterman RM, Janssen PP, Wagers SS, Wouters EF, et al. Effects of neuromuscular electrical stimulation of muscles of ambulation in patients with chronic heart failure or COPD: a systematic review of the English-language literature. Chest. 2009;136(1):44-61.

13. Sillen MJ, Franssen FM, Delbressine JM, Vaes AW, Wouters EF, Spruit MA. Efficacy of lower-limb muscle training modalities in severely dyspnoeic individuals with chronic obstructive pulmonary disease and quadriceps muscle weakness: results from the DICES trial. Thorax. 2013; in press.

14. Sillen MJ, Janssen PP, Akkermans MA, Wouters EF, Spruit MA. The metabolic response during resistance training and neuromuscular electrical stimulation (NMES) in patients with COPD, a pilot study. Respir Med. 2008;102(5):786-9.

15. Sillen MJ, Wouters EF, Franssen FM, Meijer K, Stakenborg KH, Spruit MA. Oxygen uptake, ventilation, and symptoms during low-frequency versus high-frequency NMES in COPD: a pilot study. Lung. 2011;189(1):21-6.

16. Vivodtzev I, Debigare R, Gagnon P, Mainguy V, Saey D, Dube A, et al. Functional and muscular effects of neuromuscular electrical stimulation in patients with severe COPD: a randomized clinical trial. Chest. 2012;141(3):716-25.

17. Borges $\mathrm{O}$. Isometric and isokinetic knee extension and flexion torque in men and women aged 20-70. Scand J Rehabil Med. 1989;21(1):45-53.

18. Ratamess NA, Alvar BA, Evetoch TK, Housh TJ, Kibler WB, Kraemer WJ, et al. Progression Models in Resistance Training for Healthy Adults. Medicine \& Science in Sports \& Exercise. 2009:687-708.

19. Spruit MA, Gosselink R, Troosters T, De Paepe K, Decramer M. Resistance versus endurance training in patients with COPD and peripheral muscle weakness. The European respiratory journal : official journal of the European Society for Clinical Respiratory Physiology. 2002;19(6):1072-8.

20. Revill SI, Robinson JO, Rosen M, Hogg MI. The reliability of a linear analogue for evaluating pain. Anaesthesia. 1976;31(9):1191-8. Epub 1976/11/01. 
21. Wegmann H. The "new" Declaration of Helsinki. Journal of International Biotechnology Law. 2009;6(4):173-6.

22. Sillen MJ, Franssen FM, Delbressine JM, Uszko-Lencer NH, Vanfleteren LE, Rutten EP, et al. Heterogeneity in clinical characteristics and co-morbidities in dyspneic individuals with COPD GOLD D: findings of the DICES trial. Respir Med. 2013;107(8):1186-94.

23. Hernandes NA, Wouters EF, Meijer K, Annegarn J, Pitta F, Spruit MA. Reproducibility of 6-minute walking test in patients with COPD. Eur Respir J. 2011;38(2):261-7. Epub 2010/12/24.

24. Hul van 't A, Gosselink R, Kwakkel G. Constant-load cycle endurance performance: test-retest reliability and validity in patients with COPD. J Cardiopulm Rehabil. 2003;23(2):143-50.

25. Theurel J, Lepers R, Pardon L, Maffiuletti NA. Differences in cardiorespiratory and neuromuscular responses between voluntary and stimulated contractions of the quadriceps femoris muscle. Respiratory physiology \& neurobiology. 2007;157(2-3):341-7.

26. Giavedoni S, Deans A, McCaughey P, Drost E, MacNee W, Rabinovich RA. Neuromuscular electrical stimulation prevents muscle function deterioration in exacerbated COPD: a pilot study. Respir Med. 2012;106(10):1429-34

27. Abdellaoui A, Prefaut C, Gouzi F, Couillard A, Coisy-Quivy M, Hugon G, et al. Skeletal muscle effects of electrostimulation after COPD exacerbation: a pilot study. Eur Respir J. 2011;38(4):781-8.

28. Troosters T, Probst VS, Crul T, Pitta F, Gayan-Ramirez G, Decramer M, et al. Resistance training prevents deterioration in quadriceps muscle function during acute exacerbations of chronic obstructive pulmonary disease. Am J Respir Crit Care Med. 2010;181(10):1072-7.

29. Zanotti E, Felicetti G, Maini M, Fracchia C. Peripheral muscle strength training in bed-bound patients with COPD receiving mechanical ventilation: effect of electrical stimulation. Chest. 2003;124(1):292-6.

30. Maffiuletti NA, Roig M, Karatzanos E, Nanas S. Neuromuscular electrical stimulation for preventing skeletal-muscle weakness and wasting in critically ill patients: a systematic review. BMC medicine. 2013;11:137.

31. Nuhr M, Crevenna R, Gohlsch B, Bittner C, Pleiner J, Wiesinger G, et al. Functional and biochemical properties of chronically stimulated human skeletal muscle. Eur J Appl Physiol. 2003;89(2):202-8.

32. Theriault R, Boulay MR, Theriault G, Simoneau JA. Electrical stimulation-induced changes in performance and fiber type proportion of human knee extensor muscles. Eur J Appl Physiol Occup Physiol. 1996;74(4):311-7.

33. Gondin J, Brocca L, Bellinzona E, D'Antona G, Maffiuletti NA, Miotti D, et al. Neuromuscular electrical stimulation training induces atypical adaptations of the human skeletal muscle phenotype: a functional and proteomic analysis. J Appl Physiol. 2011;110(2):433-50.

34. Perez M, Lucia A, Rivero JL, Serrano AL, Calbet JA, Delgado MA, et al. Effects of transcutaneous shortterm electrical stimulation on $\mathrm{M}$. vastus lateralis characteristics of healthy young men. Pflugers Arch. 2002;443(5-6):866-74

35. Tesch PA, Thorsson A, Essen-Gustavsson B. Enzyme activities of FT and ST muscle fibers in heavyresistance trained athletes. J Appl Physiol. 1989;67(1):83-7.

36. Kryger Al, Andersen JL. Resistance training in the oldest old: consequences for muscle strength, fiber types, fiber size, and MHC isoforms. Scand J Med Sci Sports. 2007;17(4):422-30.

37. Andersen JL, Aagaard P. Myosin heavy chain IIX overshoot in human skeletal muscle. Muscle Nerve. 2000;23(7):1095-104.

38. Farup J, Kjolhede T, Sorensen H, Dalgas U, Moller AB, Vestergaard PF, et al. Muscle morphological and strength adaptations to endurance vs. resistance training. J Strength Cond Res. 2012;26(2):398-407.

39. Verdijk LB, Gleeson BG, Jonkers RA, Meijer K, Savelberg HH, Dendale P, et al. Skeletal muscle hypertrophy following resistance training is accompanied by a fiber type-specific increase in satellite cell content in elderly men. J Gerontol A Biol Sci Med Sci. 2009;64(3):332-9. 


\section{SUPPLEMENTAL TABLE}

Table S8.1 Characteristics of metabolic group and remaining group.

\begin{tabular}{|c|c|c|c|c|}
\hline & & $\begin{array}{c}\text { Metabolic load group } \\
n=24\end{array}$ & $\begin{array}{c}\text { Remaining group } \\
\mathrm{n}=96\end{array}$ & P-value \\
\hline Gender & Male/Female & $14 / 10$ & $48 / 48$ & 0.467 \\
\hline Age & years & $65.3 \pm 1.5$ & $64.7 \pm 0.9$ & 0.765 \\
\hline $\mathrm{FEV}_{1}$ & liters & $0.95 \pm 0.05$ & $0.82 \pm 0.04$ & 0.104 \\
\hline $\mathrm{FEV}_{1}$ & $\%$ predicted & $38 \pm 3$ & $32 \pm 1$ & 0.048 \\
\hline $\mathrm{FEV}_{1} / \mathrm{VC} \max$ & $\%$ & $34 \pm 2$ & $31 \pm 1$ & 0.288 \\
\hline $\mathrm{DL}_{\mathrm{CO}}$ & $\%$ & $46 \pm 3$ & $40 \pm 2$ & 0.102 \\
\hline RV & $\%$ & $186 \pm 12$ & $202 \pm 6$ & 0.207 \\
\hline $\mathrm{PaO}_{2}$ & $\mathrm{kPa}$ & $9.7 \pm 0.2$ & $9.7 \pm 0.2$ & 0.932 \\
\hline $\mathrm{PaCO}_{2}$ & $\mathrm{kPa}$ & $5.2 \pm 0.1$ & $5.8 \pm 0.1$ & 0.001 \\
\hline $\mathrm{SpO}_{2}$ & $\%$ & $95.9 \pm 0.4$ & $95.51 \pm 0.3$ & 0.181 \\
\hline Peak load & watts & $46 \pm 3$ & $44 \pm 1$ & 0.511 \\
\hline Peak load & $\%$ predicted & $42 \pm 6$ & $40 \pm 2$ & 0.714 \\
\hline Bodyweight & kg & $74 \pm 3$ & $68 \pm 2$ & 0.105 \\
\hline $\mathrm{BMI}$ & $\mathrm{kg} / \mathrm{m}^{2}$ & $26.1 \pm 1.0$ & $24.5 \pm 0.5$ & 0.171 \\
\hline FFMI & $\mathrm{kg} / \mathrm{m}^{2}$ & $17.2 \pm 0.4$ & $16.3 \pm 0.2$ & 0.037 \\
\hline Peak torque & $\mathrm{Nm}$ & $86.6 \pm 5.2$ & $73.6 \pm 2.7$ & 0.030 \\
\hline Peak torque & $\%$ predicted & $59 \pm 3$ & $53 \pm 2$ & 0.102 \\
\hline Total work & Joules & $1389 \pm 95$ & $1122 \pm 49$ & 0.015 \\
\hline 1RM leg extension & $\mathrm{kg}$ & $35 \pm 3$ & $30 \pm 1$ & 0.043 \\
\hline 1RM leg press & $\mathrm{kg}$ & $78 \pm 8$ & $66 \pm 3$ & 0.134 \\
\hline
\end{tabular}

Values expressed as mean $\pm \mathrm{SEM}$. $\mathrm{FEV}_{1}=$ forced expiratory volume in one second; $\mathrm{VC}$ max=maximum vital capacity; $\mathrm{DL}_{\mathrm{CO}}=$ diffusion capacity of the lung for carbon monoxide; $\mathrm{RV}=$ residual volume; $\mathrm{PaO}_{2}=$ resting arterial oxygen tension; $\mathrm{PaCO}_{2}=$ resting arterial carbon dioxide tension; $\mathrm{SpO}_{2}=$ transcutaneous oxygen saturation; $\mathrm{BMI}=$ body mass index; $\mathrm{FFMI}=$ fat-free mass index; $1 \mathrm{RM}=$ one-repetition maximum; $\mathrm{Nm}=$ newtonmeter; $\mathrm{kPa}=$ kilopascal; $\mathrm{ml} / \mathrm{min}=$ milliliter per minute; $\mathrm{kg} / \mathrm{m}^{2}=\mathrm{kilogram}$ per squared meter. 


\section{CHAPTER 9}

General discussion 
Chapter 9 


\section{INTRODUCTION}

Although defined by the presence of chronic airflow limitation, extra-pulmonary manifestations are recognized as important contributors to morbidity and mortality in patients with chronic obstructive pulmonary disease (COPD) (1,2). Skeletal muscle dysfunction has been identified as a clinical feature beyond the lungs which is related to impairment and disability in these patients (3). Peripheral muscle strength is correlated with daily physical activity levels which are low compared to healthy controls (4). Pulmonary rehabilitation is an important non-pharmacological treatment for patients with COPD, resulting in reduction in symptom burden, increased exercise performance and enhanced participation in everyday activities (5). Exercise-based rehabilitation programs typically consist of whole-body endurance or interval training (6). Neuromuscular electrical stimulation (NMES) is a relative new intervention in pulmonary rehabilitation to counteract muscle weakness and improve exercise performance $(7,8)$ in patients with high symptom burden and severe muscle weakness. The central aim of this thesis was to investigate the safety, efficacy and metabolic and structural effects of NMES in dyspneic individuals with COPD. In this chapter changes in skeletal muscle and exercise capacity following traditional skeletal muscle training and NMES of the lower extremities will be discussed. Also, the metabolic load during these interventions will be considered. Finally, future directions and recommendations for future research will be described.

\section{EFFECTS OF TRADITIONAL SKELETAL MUSCLE TRAINING IN COPD}

Lower-limb skeletal muscle training in patients with COPD mostly consists of endurance training, interval training, strength training and/or NMES (7). Endurance training, the most commonly applied exercise modality in pulmonary rehabilitation programs, aims to condition the lower-limb muscles and improve cardio-respiratory fitness (9). Interval training is an alternative to standard endurance training in these patients (6). This training is a modification of endurance training where high-intensity exercise is interspersed regularly with periods of lower intensity exercise (5). Both training modalities significantly improved exercise capacity and health status in patients with COPD without significant differences between both modalities (6). Because of the severity of exercise-induced dyspnea it may be difficult to achieve the target training intensity or training time for some patients (10). This may result in unintended rests (11) and suboptimal outcomes. For these patients, strength training is an alternative training modality. Compared to endurance training, strength training results in less dyspnea and a lower cardiopulmonary demand during exercise (12). Lower oxygen consumption and minute ventilation have been shown during strength training 
compared with endurance training (cycling, walking and stair climbing) in patients with COPD. Strength training is exercise that causes muscles to work or hold against an applied force or weight (13). O'Shea and colleagues systematically reviewed the effects of strength training in individuals with COPD, including 18 controlled clinical trials, with appreciable increases in muscle strength (14). Movements reflecting daily tasks, such as stair-climbing and sit-to-stand, improved significantly following strength training compared with no intervention or aerobic training (14). Another advantage of strength training compared to endurance training is that it led to similar $(15)$ or larger $(16,17)$ improvements in skeletal muscle strength in patients with COPD. Despite improvements in muscle strength after progressive muscle strength training, inconclusive evidence was found for changes in skeletal muscle structure (14).

\section{NMES AS AN ALTERNATIVE TRAINING MODALITY}

Next to strengthening exercises, NMES is a feasible training modality associated with decreased perception of dyspnea (18). NMES can be applied as a complement intervention to voluntary exercise training in disabled patients or can be used as an intervention in patients who are bedbound and/or unable to perform volitional exercises $(19,20)$. To gain insight into the currently available literature on the effects following NMES, two systematic reviews were performed as part of the current thesis using the databases Medline/PubMed, EMBASE, Cochrane Central Register of Controlled Trials, CINAHL and The Physical Therapy Evidence Database (PEDro) (chapters 2 and 3). In chapter 2, the effects of NMES in patients with COPD or congestive heart failure were systematically reviewed. Most of the COPD studies reported significant improvements in muscle strength, exercise capacity and/or health status. The improvements following NMES were clinically relevant. The mean difference in change in the incremental shuttle walk test distance between NMES and sham stimulation exceeded the minimal clinically important difference of 47 meters and the mean difference in change in the chronic respiratory dyspnea questionnaire domain "dyspnea" of 1.2 points per question also exceeded the minimal clinically important difference of 0.5 points per question. These findings have recently been confirmed in a meta-analysis studying NMES in adults with advanced disease and muscle weakness (22). In adults with progressive diseases such as COPD, chronic heart failure and cancer NMES appeared to be an effective means of improving muscle weakness (21).

To date, various studies focussed on the effects of NMES on gains in lower-limb muscle performance, activation of motor units and/or muscle energetics (8,22-24). However, the effects of NMES on intramuscular changes had not been systematically reviewed at the start of the current project. NMES can be distinguished in high-frequency NMES (HF-NMES, $\geq 50$ Hertz) and low-frequency NMES (LF-NMES, $\leq 20$ Hertz) $(8,22,25-27)$. The 
systematic review on metabolic and structural changes in lower-limb skeletal muscle following NMES (chapter 3 ) reported significant increases in oxidative enzyme activity following LF-NMES (28-33) while muscle size increased significantly in 6 of the 11 included HF-NMES studies.

However, NMES protocols varied tremendously among the trials in abovementioned systematic reviews (chapters 2 and 3). Pulse duration ranged between 200 and $700 \mu \mathrm{s}$ and the duration of the NMES programs varied between 10 minutes to 8 hours/day, 1 to 2 sessions/day, 3 to 7 days/week for 4 to 10 weeks. Furthermore, the external validity of the findings of the reviewed trials were also limited because of the variability in study participants. Therefore, these reviews recommended that future trials are needed to determine the optimal settings of NMES, such as stimulation frequency (HF-NMES or LF-NMES) and should not only include healthy persons but also persons who are unable to perform or complete volitional exercise training programs. Also, both reviews suggested that larger well-designed trials determining the optimal parameters for a NMES program are needed to improve our understanding of NMES.

\section{NMES VERSUS STRENGTH TRAINING IN PATIENTS WITH COPD}

In chapter 7, the effects of strength training and NMES were head-to-head compared in a randomized controlled trial (RCT) in severely dyspneic COPD patients with muscle weakness referred for pulmonary rehabilitation. Endurance training was in the RCT not compared with strength training and/or NMES, because it may be difficult to achieve the target training intensity or training time during endurance training for some patients due to exercise-induced dyspnea (13). Quadriceps muscle strength increased following HF-NMES or strength training, but not following LF-NMES (chapter 7). Quadriceps muscle endurance, 6-min walk distance, endurance cycling time, mMRC dyspnea grade, symptoms of depression, problematic activities of daily living and health status improved compared to baseline following all three training modalities (HF-NMES, LF-NMES and strength training) without differences between groups (chapter 7). HF-NMES was as effective as strength training in individuals with COPD and disabling dyspnea (chapter 7).

In all training modalities, exercise capacity improved significantly compared to baseline without significant between-group differences. Because of the significant increases in oxidative enzyme activity following LF-NMES (28-33) (chapter 3), it was reasonable to hypothesize that exercise performance and muscle endurance would improve to a greater extent following LF-NMES compared to HF-NMES or strength training in this trial (chapter 7). However, improvements in exercise performance, measured with the 6-min walk test and cycle time during the constant work rate test, did not significantly 
differ between groups. Improvements in exercise performance in LF-NMES may be attributable to changes in muscle fiber type composition and an increased oxidative enzyme activity. Indeed, in healthy volunteers and patients with chronic heart failure citrate synthase, a marker enzyme of the tricarboxylic acid cycle, increased following LF-NMES $(15 \mathrm{~Hz})$ (31,34). Moreover, LF-NMES resulted in endurance-like metabolic alterations, such as an increased ATP utilization (35) and an enhanced glucose uptake (36). The increased exercise performance in HF-NMES can probably be explained by the non-selective recruitment of both type I and type IIx fibers during HF-NMES (37) and partially by recruitment through central pathways producing contractions according to the size principle activating the slower before the faster motor units (38). Strength training had already been shown to increase exercise capacity, with no significant differences compared to endurance training (15).

It would have been plausible to hypothesize that gains in muscle strength in the RCT were related to an increased muscle mass. However, fat-free mass did not change, while muscle function significantly improved in the current study (chapter 7). Thus, improvements in muscle function and exercise performance following HF-NMES or strength training are probably due to intramuscular adaptations (39). Improvements in muscle function without muscle hypertrophy following HF-NMES may be attributed to an increased fiber proportion associating with metabolic and morphological adaptations involving an increase in both the oxidative capacity and capillarization of fast-twitch fibers (40) and following strength training in increased levels of glycolytic enzymes (39). Neural adaptations may also contribute to an improved muscle function without muscle hypertrophy following HF-NMES or strength training (41-43). Alterations in synergistic activation and reductions in antagonist activation are neural factors that have been identified as changing during the early stages of strength training which could contribute to maximal force generation (44). Neural adaptations to strength training can also mediate increases in muscle strength and these include changes in motor unit activation, motoneuron excitability and spinal reflex mechanisms (43). The impact of NMES is dependent on the training duration. It is well known that long training duration is needed to induce muscle hypertrophy (45).

The systematic reviews (chapters 2 and 3) suggested that larger well-designed trials determining the optimal parameters for a NMES program are needed in order to improve our understanding of NMES. Nevertheless, there is a sort of agreement on some NMES parameters (8). In the RCT (chapter 7) a pulse duration of $400 \mu$ s was used because pulse durations of $300-400 \mu$ s are generally recommended for stimulating large muscles, such as the quadriceps or calf muscles (46). It is crucial to motivate the subject to tolerate the highest current intensity, although there is a wide variability in the course of NMES intensity. This largely depends on the subject's tolerance of the current, the skin blood flow and the thickness of the subcutaneous fat layer $(8,47)$. In 
the current thesis, carbon-rubber electrodes in moistened sponges were used instead of self-adhesive electrodes to minimize skin nociceptive sensations and to evoke the greatest torque by applying the highest current through the lowest impedance (48).

In the trial described in chapter 7 of this thesis, muscle strength significantly increased in the HF-NMES group and the strength training group, while exercise performance and health status significantly increased in all intervention groups. Several studies indicate that higher intensities in NMES are associated with better improvements following NMES training (49-52). Moreover, two HF-NMES studies in severely disabled patients with COPD showed clear relations between the change in muscle strength and the change in the intensity of NMES $(53,54)$. On the other hand, Lloyd and colleagues showed a considerable inter-individual variation in response to NMES exercise, which may relate more to the characteristics of the subject than to NMES parameters (55). Indeed, Lieber and Kelly suggested that NMES effectiveness depends on intrinsic anatomical properties, such as individual motor nerve branching, which determines the response of the muscle to the application of electrical current over the skin (48). Furthermore, the findings of chapter 6 emphasize that COPD is a heterogeneous disease with a variable clinical presentation, even in individuals with very severe COPD and a comparable degree of dyspnea, classified as GOLD group D (56). Significant gender differences were found for exercise performance, lower-limb muscle function and various comorbidities. Although the inclusion criteria of this trial limit the external validity of these findings (chapter 6), previous studies had already shown a large heterogeneity in clinical outcomes in less severe patients with COPD after stratification dyspnea grade or GOLD stage $(57,58)$.

Lower-limb muscle dysfunction in patients with COPD, a major extra-pulmonary manifestation, is the consequence of complex interactions between local and systemic factors (59). Some of these factors can be partially reversed by muscle training, such as muscle fiber atrophy, capillary density and early lactate release (60). However, other factors, such as hypoxemia and systemic inflammation, persist after training and are most probably of systemic nature, including i.e. comorbidities, exacerbations and gas exchange abnormalities (60). Thus, the response to training is probably the consequence of inter-individual variation between persons with COPD, including anatomical and pathophysiological factors.

\section{METABOLIC LOAD DURING NMES OR STRENGTH TRAINING}

Chapters 4, 5 and 8 of this thesis focused on the metabolic load of the various local skeletal muscle training modalities in patients with COPD. Peak oxygen uptake and ventilation in the RCT (chapter 8 ) were comparable to the pilot studies (chapters 4 and 5 ) and did not change over time. Because of the constant metabolic load in 
combination with stable symptom scores for dyspnea, fatigue and muscle pain over time it seems reasonable to hypothesize that the improvements in muscle function are at least partially due to intramuscular changes. A major limitation of chapters 4 and 5 is that the metabolic load was only measured during one single session of NMES or strength training. Other limitations of these measurements are the small sample sizes and that selected patient characteristics may limit the external validity and broad applicability of the findings. Only patients without long-term oxygen therapy were eligible to participate due to the methodology used (12). The low metabolic load explains the applicability of these interventions in severely dyspneic patients, even during acute COPD exacerbations $(53,61,62)$.

\section{LIMITATIONS AND FUTURE DIRECTIONS}

In the RCT studying the efficacy of different NMES modalities or strength training in severely dyspneic individuals with COPD (chapter 7), outcome assessors were blinded for group allocation to avoid detection bias. Individuals randomly assigned to HF-NMES or LF-NMES were also blinded for the stimulation frequency applied. However, the RCT did not include a usual care or NMES sham control group. Previously, NMES and strength training had been shown to improve muscle strength, exercise performance and quality of life to a greater extent than a control group or sham NMES $(14,63,64)$. The interventions were part of an 8-week interdisciplinary pulmonary rehabilitation program, so it remains unclear whether and to what extent the other rehabilitative interventions may have contributed to the improvements during the trial. Nonetheless, determining factors that could explain the improved effects and the wide variability in training response are still missing. Although muscle biopsies are lacking, it seems reasonable to hypothesize that the improvements in muscle function and exercise performance were at least partially due to intramuscular changes. Another limitation is that a per protocol instead of intention-to-treat analysis was conducted considering the $25 \%$ drop out rate. However, the drop-out rate was random across the sample, did not differ between groups and was comparable with published strength training trials (range of drop-out rates from 11 to 38\%) (14). Moreover, the 29 individuals that did not complete the trial had comparable baseline values compared to the subjects that did complete the trial. In the current analyses, all participants who had their outcome measures assessed were included in the analysis, regardless of the number of sessions they successfully completed. No imputations were made for missing data.

The usage of NMES has several limitations. One limitation is that NMES imposes a continuous contractile activity to the same population of superficial muscle fibers, those with the axonal branches in proximity to the stimulating electrodes (65). Then again, if pulse amplitude is progressively increased throughout the NMES session (66), new fibers located at a greater distance from the electrode could be depolarized, while 
the superficial fibers maintain their contractile activity (67). It has also been suggested that as stimulations are delivered at a fixed joint angle (68), the effects of NMES are considered to be poorly related to functional activities of daily living (69) which is in contrary with the RCT showing improvements in problematic activities of daily life in all training groups (chapter 7). Another limitation of NMES is related to the long-term effectiveness following discontinuation. Only few studies have reported the effects of NMES after follow-up. However, these studies are limited to patients with neurological disorders such as cerebral palsy $(71)$ or hemiplegia $(72,73)$ in which upper limb muscles were electrically stimulated. The main conclusion related to the long-term effects of these studies is that NMES may not be a long term intervention for muscle re-education or restoration of movement (74). Moreover, NMES is a passive intervention in which subjects are seated in a comfortable chair, but on the other hand it is recommended to stimulate subjects to a more active lifestyle. So, it is essential to add self-management strategies and ways to translate gains in exercise capacity into increased physical activity in these subjects (5). Advantages of NMES are that it is safe, generally welltolerated, relatively cheap, it is applicable in very disabled patients and subjects can self-administer NMES in the home environment $(21,64)$.

Despite the methodological considerations, some conclusions can be drawn. First, HFNMES, LF-NMES and strength training are feasible, safe and effective training modalities in severely dyspneic individuals with COPD, even in a population characterized by comorbidities and exacerbations. Secondly, LF-NMES seems to be a training modality resulting in changes in oxidative enzyme activity. Finally, the metabolic load remains relatively low during HF-NMES, LF-NMES and strength training with increasing training loads over time.

Till present, NMES has been used for the preservation or recovery of muscle mass and function during prolonged periods of disuse or immobilization in orthopedic patient populations (patients with anterior cruciate ligament reconstruction or bone fracture), or for the improvement of muscle function in different healthy study subjects $(19,52,70-73)$. NMES has been shown to be a successful intervention in different patient populations, such as patients with chronic heart failure or COPD (chapter 2), orthopedic patients (74), neurological patients $(75)$ or critically ill patients $(76,77)$. It depends on the severity of dyspnea, status of the muscle and the therapeutic goals which intervention is designated. To our opinion, the usage of NMES is the preferred training modality for all people and patients who are bed-bound and/or are unable to perform regular training or rehabilitation programs, for example patients in the intensive care unit or patients undergoing major surgery, dialysis or chemotherapy. Compared to strength training, HF-NMES may be the preferred intervention if patients are partially or not able to perform volitional exercises because of dyspnea, fatigue or other symptoms. LF-NMES is only a preferred training modality in patients with a preserved peripheral muscle strength and if the main goal is to increase exercise capacity. If patients are able to improve their muscle function volitionally, muscle 
strength training is the designated intervention. Large well-designed trials are needed to study the feasibility and effects of NMES in abovementioned patient populations. It may also be worthwhile to study other large lower extremity muscle groups, for example gluteus medius and minimus muscles (78), as to date in the majority of the studies mostly the quadriceps femoris muscles have been electrically stimulated. Finally, metabolic and structural changes following NMES should also be studied to gain insight into the physiological mechanisms following NMES. 


\section{REFERENCES}

1. Patel AR, Hurst JR. Extrapulmonary comorbidities in chronic obstructive pulmonary disease: state of the art. Expert review of respiratory medicine. 2011;5(5):647-62.

2. Vanfleteren LE, Spruit MA, Groenen M, Gaffron S, van Empel VP, Bruijnzeel PL, et al. Clusters of comorbidities based on validated objective measurements and systemic inflammation in patients with chronic obstructive pulmonary disease. Am J Respir Crit Care Med. 2013;187(7):728-35.

3. Skeletal muscle dysfunction in chronic obstructive pulmonary disease. A statement of the American Thoracic Society and European Respiratory Society. Am J Respir Crit Care Med. 1999;159(4 Pt 2):S1-40.

4. Pitta F, Troosters T, Spruit MA, Probst VS, Decramer M, Gosselink R. Characteristics of physical activities in daily life in chronic obstructive pulmonary disease. Am J Respir Crit Care Med. 2005;171(9):972-7.

5. Spruit MA, Singh SJ, Garvey C, Zuwallack R, Nici L, Rochester C, et al. An official american thoracic society/european respiratory society statement: key concepts and advances in pulmonary rehabilitation. Am J Respir Crit Care Med. 2013;188(8):e13-64.

6. Beauchamp MK, Nonoyama M, Goldstein RS, Hill K, Dolmage TE, Mathur S, et al. Interval versus continuous training in individuals with chronic obstructive pulmonary disease--a systematic review. Thorax. 2010;65(2):157-64.

7. Spruit MA, Wouters EF. New modalities of pulmonary rehabilitation in patients with chronic obstructive pulmonary disease. Sports Med. 2007;37(6):501-18.

8. Vanderthommen M, Duchateau J. Electrical stimulation as a modality to improve performance of the neuromuscular system. Exerc Sport Sci Rev. 2007;35(4):180-5.

9. Lacasse Y, Goldstein R, Lasserson TJ, Martin S. Pulmonary rehabilitation for chronic obstructive pulmonary disease. Cochrane Database Syst Rev. 2006(4):CD003793.

10. Maltais F, LeBlanc P, Jobin J, Berube C, Bruneau J, Carrier L, et al. Intensity of training and physiologic adaptation in patients with chronic obstructive pulmonary disease. Am J Respir Crit Care Med. 1997;155(2):555-61.

11. Puhan MA, Busching G, Schunemann HJ, VanOort E, Zaugg C, Frey M. Interval versus continuous highintensity exercise in chronic obstructive pulmonary disease: a randomized trial. Ann Intern Med. 2006;145(11):816-25.

12. Probst VS, Troosters T, Pitta F, Decramer M, Gosselink R. Cardiopulmonary stress during exercise training in patients with COPD. Eur Respir J. 2006;27(6):1110-8.

13. American College of Sports Medicine Position Stand. Exercise and physical activity for older adults. Med Sci Sports Exerc. 1998;30(6):992-1008.

14. O'Shea SD, Taylor NF, Paratz JD. Progressive resistance exercise improves muscle strength and may improve elements of performance of daily activities for people with COPD: a systematic review. Chest. 2009;136(5):1269-83.

15. Spruit MA, Gosselink R, Troosters T, De Paepe K, Decramer M. Resistance versus endurance training in patients with COPD and peripheral muscle weakness. Eur Respir J. 2002;19(6):1072-8.

16. Normandin EA, McCusker C, Connors M, Vale F, Gerardi D, ZuWallack RL. An evaluation of two approaches to exercise conditioning in pulmonary rehabilitation. Chest. 2002;121(4):1085-91.

17. Ortega F, Toral J, Cejudo P, Villagomez R, Sanchez H, Castillo J, et al. Comparison of effects of strength and endurance training in patients with chronic obstructive pulmonary disease. Am J Respir Crit Care Med. 2002;166(5):669-74.

18. Bausewein C, Booth S, Gysels M, Higginson I. Non-pharmacological interventions for breathlessness in advanced stages of malignant and non-malignant diseases. Cochrane Database Syst Rev. 2008(2):CD005623.

19. Maffiuletti NA. Physiological and methodological considerations for the use of neuromuscular electrical stimulation. Eur J Appl Physiol. 2010;110(2):223-34.

20. Hainaut K, Duchateau J. Neuromuscular electrical stimulation and voluntary exercise. Sports Med. 1992;14(2):100-13.

21. Maddocks M, Gao W, Higginson IJ, Wilcock A. Neuromuscular electrical stimulation for muscle weakness in adults with advanced disease. Cochrane Database Syst Rev. 2013;1:CD009419. 
22. Maffiuletti NA. Physiological and methodological considerations for the use of neuromuscular electrical stimulation. Eur J Appl Physiol. 2010.

23. Gregory CM, Bickel CS. Recruitment patterns in human skeletal muscle during electrical stimulation. Phys Ther. 2005;85(4):358-64.

24. Gondin J, Cozzone PJ, Bendahan D. Is high-frequency neuromuscular electrical stimulation a suitable tool for muscle performance improvement in both healthy humans and athletes? Eur J Appl Physiol. 2011;111(10):2473-87.

25. Chan Kwan Kit-Lan P. Contemporary trends in electrical stimulation: "The frequency-specificity theory". Hong-Kong Physiother J. 1991/1992;13:23-7.

26. Vivodtzev I, Lacasse $Y$, Maltais F. Neuromuscular electrical stimulation of the lower limbs in patients with chronic obstructive pulmonary disease. J Cardiopulm Rehabil Prev. 2008;28(2):79-91.

27. Grimby L, Hannerz J. Firing rate and recruitment order of toe extensor motor units in different modes of voluntary conraction. J Physiol. 1977;264(3):865-79.

28. Gauthier JM, Theriault R, Theriault G, Gelinas $Y$, Simoneau JA. Electrical stimulation-induced changes in skeletal muscle enzymes of men and women. Med Sci Sports Exerc. 1992;24(11):1252-6.

29. Theriault R, Boulay MR, Theriault G, Simoneau JA. Electrical stimulation-induced changes in performance and fiber type proportion of human knee extensor muscles. Eur J Appl Physiol Occup Physiol. 1996;74(4):311-7.

30. Theriault R, Theriault G, Simoneau JA. Human skeletal muscle adaptation in response to chronic lowfrequency electrical stimulation. J Appl Physiol. 1994;77(4):1885-9.

31. Nuhr M, Crevenna R, Gohlsch B, Bittner C, Pleiner J, Wiesinger G, et al. Functional and biochemical properties of chronically stimulated human skeletal muscle. Eur J Appl Physiol. 2003;89(2):202-8.

32. Gondin J, Brocca L, Bellinzona E, D'Antona G, Maffiuletti NA, Miotti D, et al. Neuromuscular electrical stimulation training induces atypical adaptations of the human skeletal muscle phenotype: a functional and proteomic analysis. J Appl Physiol. 2011;110(2):433-50.

33. Quittan M, Wiesinger GF, Sturm B, Puig S, Mayr W, Sochor A, et al. Improvement of thigh muscles by neuromuscular electrical stimulation in patients with refractory heart failure: a single-blind, randomized, controlled trial. Am J Phys Med Rehabil. 2001;80(3):206-14; quiz 15-6, 24.

34. Nuhr MJ, Pette D, Berger R, Quittan M, Crevenna R, Huelsman M, et al. Beneficial effects of chronic low-frequency stimulation of thigh muscles in patients with advanced chronic heart failure. Eur Heart J. 2004;25(2):136-43.

35. Bergstrom M, Hultman E. Energy cost and fatigue during intermittent electrical stimulation of human skeletal muscle. J Appl Physiol. 1988;65(4):1500-5.

36. Hamada T, Sasaki H, Hayashi T, Moritani T, Nakao K. Enhancement of whole body glucose uptake during and after human skeletal muscle low-frequency electrical stimulation. J Appl Physiol. 2003;94(6): 2107-12.

37. Bickel CS, Gregory CM, Dean JC. Motor unit recruitment during neuromuscular electrical stimulation: a critical appraisal. Eur J Appl Physiol. 2011;111(10):2399-407.

38. Bergquist AJ, Clair JM, Lagerquist O, Mang CS, Okuma Y, Collins DF. Neuromuscular electrical stimulation: implications of the electrically evoked sensory volley. Eur J Appl Physiol. 2011;111(10):2409-26.

39. Tesch PA, Thorsson A, Essen-Gustavsson B. Enzyme activities of FT and ST muscle fibers in heavyresistance trained athletes. J Appl Physiol. 1989;67(1):83-7.

40. Perez M, Lucia A, Rivero JL, Serrano AL, Calbet JA, Delgado MA, et al. Effects of transcutaneous shortterm electrical stimulation on $\mathrm{M}$. vastus lateralis characteristics of healthy young men. Pflugers Arch. 2002;443(5-6):866-74.

41. Hortobagyi T, Maffiuletti NA. Neural adaptations to electrical stimulation strength training. Eur J Appl Physiol. 2011;111(10):2439-49.

42. Aagaard P, Simonsen EB, Andersen JL, Magnusson P, Dyhre-Poulsen P. Neural adaptation to resistance training: changes in evoked V-wave and H-reflex responses. J Appl Physiol. 2002;92(6):2309-18.

43. Carroll TJ, Riek S, Carson RG. Neural adaptations to resistance training: implications for movement control. Sports Med. 2001;31(12):829-40.

44. Griffin L, Cafarelli E. Resistance training: cortical, spinal, and motor unit adaptations. Can J Appl Physiol. 2005;30(3):328-40. 
45. American College of Sports Medicine position stand. Progression models in resistance training for healthy adults. Med Sci Sports Exerc. 2009;41(3):687-708.

46. Bowman BR, Baker LL. Effects of waveform parameters on comfort during transcutaneous neuromuscular electrical stimulation. Ann Biomed Eng. 1985;13(1):59-74.

47. Petrofsky JS, Suh HJ, Gunda S, Prowse M, Batt J. Interrelationships between body fat and skin blood flow and the current required for electrical stimulation of human muscle. Medical engineering \& physics. 2008;30(7):931-6.

48. Lieber RL, Kelly MJ. Factors influencing quadriceps femoris muscle torque using transcutaneous neuromuscular electrical stimulation. Phys Ther. 1991;71(10):715-21; discussion 22-3.

49. Lai HS, De Domenico G, Strauss GR. The effect of different electro-motor stimulation training intensities on strength improvement. Aust J Physiother. 1988;34:151-64.

50. Selkowitz DM. Improvement in isometric strength of the quadriceps femoris muscle after training with electrical stimulation. Phys Ther. 1985;65(2):186-96.

51. Snyder-Mackler L, Delitto A, Stralka SW, Bailey SL. Use of electrical stimulation to enhance recovery of quadriceps femoris muscle force production in patients following anterior cruciate ligament reconstruction. Phys Ther. 1994;74(10):901-7.

52. Filipovic A, Kleinoder H, Dormann U, Mester J. Electromyostimulation--a systematic review of the influence of training regimens and stimulation parameters on effectiveness in electromyostimulation training of selected strength parameters. J Strength Cond Res. 2011;25(11):3218-38.

53. Giavedoni S, Deans A, McCaughey P, Drost E, MacNee W, Rabinovich RA. Neuromuscular electrical stimulation prevents muscle function deterioration in exacerbated COPD: a pilot study. Respir Med. 2012;106(10):1429-34.

54. Vivodtzev I, Debigare R, Gagnon P, Mainguy V, Saey D, Dube A, et al. Functional and muscular effects of neuromuscular electrical stimulation in patients with severe COPD: a randomized clinical trial. Chest. 2012;141(3):716-25.

55. Lloyd T, De Domenico G, Strauss GR, Singer K. A review of the use of electro-motor stimulation in human muscles. Aust J Physiother 1986;32:18-30.

56. Vestbo J, Hurd SS, Agusti AG, Jones PW, Vogelmeier C, Anzueto A, et al. Global strategy for the diagnosis, management, and prevention of chronic obstructive pulmonary disease: GOLD executive summary. Am J Respir Crit Care Med. 2013;187(4):347-65.

57. Agusti A, Calverley PM, Celli B, Coxson HO, Edwards LD, Lomas DA, et al. Characterisation of COPD heterogeneity in the ECLIPSE cohort. Respiratory research. 2010;11:122.

58. Spruit MA, Pennings HJ, Janssen PP, Does JD, Scroyen S, Akkermans MA, et al. Extra-pulmonary features in COPD patients entering rehabilitation after stratification for MRC dyspnea grade. Respir Med. 2007;101(12):2454-63.

59. Man WD, Kemp P, Moxham J, Polkey MI. Skeletal muscle dysfunction in COPD: clinical and laboratory observations. Clin Sci (Lond). 2009;117(7):251-64.

60. Gea J, Agusti A, Roca J. Pathophysiology of Muscle Dysfunction in Copd. J Appl Physiol. 2013.

61. Abdellaoui A, Prefaut C, Gouzi F, Couillard A, Coisy-Quivy M, Hugon G, et al. Skeletal muscle effects of electrostimulation after COPD exacerbation: a pilot study. Eur Respir J. 2011;38(4):781-8.

62. Troosters T, Probst VS, Crul T, Pitta F, Gayan-Ramirez G, Decramer M, et al. Resistance training prevents deterioration in quadriceps muscle function during acute exacerbations of chronic obstructive pulmonary disease. Am J Respir Crit Care Med. 2010;181(10):1072-7.

63. Bourjeily-Habr G, Rochester CL, Palermo F, Snyder P, Mohsenin V. Randomised controlled trial of transcutaneous electrical muscle stimulation of the lower extremities in patients with chronic obstructive pulmonary disease. Thorax. 2002;57(12):1045-9.

64. Neder JA, Sword D, Ward SA, Mackay E, Cochrane LM, Clark CJ. Home based neuromuscular electrical stimulation as a new rehabilitative strategy for severely disabled patients with chronic obstructive pulmonary disease (COPD). Thorax. 2002;57(4):333-7.

65. Vanderthommen M, Depresseux JC, Dauchat L, Degueldre C, Croisier JL, Crielaard JM. Spatial distribution of blood flow in electrically stimulated human muscle: a positron emission tomography study. Muscle Nerve. 2000;23(4):482-9. 
66. Theurel J, Lepers R, Pardon L, Maffiuletti NA. Differences in cardiorespiratory and neuromuscular responses between voluntary and stimulated contractions of the quadriceps femoris muscle. Respiratory physiology \& neurobiology. 2007;157(2-3):341-7.

67. Zory R, Boerio D, Jubeau M, Maffiuletti NA. Central and peripheral fatigue of the knee extensor muscles induced by electromyostimulation. Int J Sports Med. 2005;26(10):847-53.

68. Lieber RL, Kelly MJ. Torque history of electrically stimulated human quadriceps: implications for stimulation therapy. Journal of orthopaedic research : official publication of the Orthopaedic Research Society. 1993;11(1):131-41.

69. Enoka RM. Activation order of motor axons in electrically evoked contractions. Muscle Nerve. 2002;25(6):763-4.

70. Gibson JN, Smith K, Rennie MJ. Prevention of disuse muscle atrophy by means of electrical stimulation: maintenance of protein synthesis. Lancet. 1988;2(8614):767-70.

71. Snyder-Mackler L, Delitto A, Bailey SL, Stralka SW. Strength of the quadriceps femoris muscle and functional recovery after reconstruction of the anterior cruciate ligament. A prospective, randomized clinical trial of electrical stimulation. J Bone Joint Surg Am. 1995;77(8):1166-73.

72. Caggiano E, Emrey T, Shirley S, Craik RL. Effects of electrical stimulation or voluntary contraction for strengthening the quadriceps femoris muscles in an aged male population. J Orthop Sports Phys Ther. 1994;20(1):22-8.

73. Babault N, Cometti G, Bernardin M, Pousson M, Chatard JC. Effects of electromyostimulation training on muscle strength and power of elite rugby players. J Strength Cond Res. 2007;21(2):431-7.

74. Kim KM, Croy T, Hertel J, Saliba S. Effects of neuromuscular electrical stimulation after anterior cruciate ligament reconstruction on quadriceps strength, function, and patient-oriented outcomes: a systematic review. J Orthop Sports Phys Ther. 2010;40(7):383-91.

75. Chae J, Sheffler L, Knutson J. Neuromuscular electrical stimulation for motor restoration in hemiplegia. Topics in stroke rehabilitation. 2008;15(5):412-26.

76. Gerovasili V, Stefanidis K, Vitzilaios K, Karatzanos E, Politis P, Koroneos A, et al. Electrical muscle stimulation preserves the muscle mass of critically ill patients: a randomized study. Crit Care. 2009;13(5):R161.

77. Maffiuletti NA, Roig M, Karatzanos E, Nanas S. Neuromuscular electrical stimulation for preventing skeletal-muscle weakness and wasting in critically ill patients: a systematic review. BMC medicine. 2013;11:137.

78. Bhave A, Mont M, Tennis S, Nickey M, Starr R, Etienne G. Functional problems and treatment solutions after total hip and knee joint arthroplasty. J Bone Joint Surg Am. 2005;87 Suppl 2:9-21. 
Summary 


\section{SUMMARY}

Chronic obstructive pulmonary disease (COPD) is characterized by a persistent airflow limitation that is usually progressive and associated with an enhanced inflammatory response in the airways and lungs. Chronic symptoms of progressive dyspnea, cough and sputum production are clinical characterizations of this disease. However, COPD is nowadays considered more than a respiratory disease. Extra-pulmonary manifestations and comorbidities, such as skeletal muscle dysfunction, cardiovascular and psychological conditions are commonly observed in patients with COPD. Skeletal muscle dysfunction is directly related to exercise intolerance, increased health care utilization and mortality in patients with COPD. Pulmonary rehabilitation is the most successful strategy to maximize muscle function, minimize symptom burden, promote autonomy and increase participating in everyday activities in these patients. Unfortunately, dyspneic patients with COPD are not always able to perform high intensity endurance training due to exercise-induced dyspnea and fatigue. Strength training and transcutaneous neuromuscular electrical stimulation (NMES) may be effective alternative training modalities for improving muscle function. A distinction can be made between high-frequency (HF) and low-frequency (LF) NMES. This thesis focusses on the effects of NMES and strength training (distinguished in HF-NMES and LF-NMES) in patients with severe COPD and high symptom burden.

In chapter 2, the current literature was systematically reviewed addressing the effects of NMES applied to the lower-limbs in patients with congestive heart failure (CHF) or COPD. 14 randomized controlled trials were identified ( 9 trials examined NMES in CHF patients, and 5 in COPD patients). Many of the studies reported significant improvements in muscle strength, exercise capacity, and/or health status. From the viewpoint of this systematic review, NMES looks promising as a tool for exercise trainingin patients with CHF and COPD.

The purpose of chapter $\mathbf{3}$ was to systematically review changes in enzyme activity, muscle fiber type composition and muscle fiber size in human lower-limb skeletal muscles following NMES. 18 trials were collected and reviewed in detail, 8 trials studied changes in enzyme activities, 7 trials studied changes in muscle fiber type composition and 14 trials studied changes in muscle fiber size following NMES. Most of the LF-NMES studies reported significant increases in oxidative enzyme activity, while the results concerning changes in muscle fiber composition and muscle size were conflicting. HF-NMES significantly increased muscle size in 55\% of the studies. However, considering the small number of trials included, the large heterogeneity in stimulation parameters and differences in study populations, it was difficult to draw definitive conclusions about the effects of stimulation frequencies on muscular changes.

Because high intensity endurance training is often limited in dyspneic patients with COPD, the aim of chapters $\mathbf{4}$ and $\mathbf{5}$ was to study the metabolic load (expressed as 
oxygen consumption and ventilation) and symptoms of dyspnea and fatigue during strength training and NMES. In chapter 4 the metabolic load during a strength training session was compared to a HF-NMES session in 13 patients with COPD. Both training modalities resulted in a low metabolic load and symptoms with higher values during strength training compared with HF-NMES. In chapter $\mathbf{5}$ the metabolic load and symptoms of dyspnea and fatigue were comparable and tolerable during one session of HF-NMES (75 Hertz) and one session of LF-NMES (15 Hertz) in 17 patients with COPD. Strength training, HF-NMES and LF-NMES may be suitable training modalities to be used in severely dyspneic patients with lower-limb muscle dysfunction regarding the low metabolic load and symptom scores. A prospective randomized controlled trial (RCT) was designed to study effects of HF-NMES, LF-NMES or strength training in dyspneic COPD patients with lower-limb muscle weakness.

Chapter 6 presented an overview of the clinical heterogeneity, including gender differences, in a well-defined subgroup of individuals with COPD GOLD D. A broad range of values were found for diffusion capacity, exercise capacity, quadriceps muscle function and health status in 117 patients with a Medical Research Council (MRC) dyspnea grade 4/5 and COPD GOLD D. Problematic activities of daily life as well as objectified comorbidities also varied to a great extent. Moreover, significant gender differences were found in exercise performance, lower-limb muscle function and various comorbidities.

Chapter 7 studied prospectively the effects of HF-NMES (75 Hertz), LF-NMES (15 Hertz) or strength training incorporated in an interdisciplinary pulmonary rehabilitation programme in severely dyspneic individuals with COPD and quadriceps muscle weakness at baseline. 120 individuals with COPD were randomized to HF-NMES, LF-NMES or strength training. Quadriceps muscle strength increased following HFNMES or strength training, but not following LF-NMES. Quadriceps muscle endurance, 6-min walk distance, endurance cycling time, MRC dyspnea grade, symptoms of depression, problematic activities of daily living and health status improved compared to baseline following all three training modalities without differences between groups.

In Chapter 8 the metabolic load was assessed during these interventions at 3 time points; in the first week, the fourth week and eight week of the pulmonary rehabilitation program. Peak oxygen uptake and peak ventilation remained relatively low during HF-NMES, LF-NMES or strength training with increasing training loads over time.

In Chapter 9 the changes in skeletal muscle strength and exercise capacity following HF-NMES, LF-NMES and strength training, including the metabolic load during NMES and strength training, were put into perspective and future directions were discussed. In conclusion, HF-NMES, LF-NMES and strength training are feasible, safe and effective training modalities in severely dyspneic individuals with COPD, even in a population characterized by comorbidities and exacerbations. Increases in quadriceps muscle strength and muscle endurance are higher following HF-NMES compared to LF-NMES. 
HF-NMES is as effective as strength training in patients with severe COPD and disabling dyspnea. Finally, ventilatory responses are relatively low during HF-NMES, LF-NMES or strength training. 

Samenvatting 


\section{CHRONIC OBSTRUCTIVE PULMONARY DISEASE}

Chronic obstructive pulmonary disease (COPD) betekent in het Nederlands "chronische obstructieve longziekten" en is een verzamelnaam voor longemfyseem en chronische bronchitis. Deze ziekte wordt gekarakteriseerd door een blijvende progressieve luchtwegvernauwing en gaat gepaard met ontstekingsreacties in de luchtwegen en de longen. Deze ontstekingsreacties ontstaan door langdurige blootstelling aan sigarettenrook of andere schadelijke gassen. Chronische symptomen van progressieve kortademigheid, hoesten en slijmproductie zijn klinische kenmerken van de ziekte. COPD is echter meer dan een aandoening van de luchtwegen. De afgelopen decennia is duidelijk geworden dat het een systemische aandoening is met verschijnselen buiten de longen, zoals verminderd functioneren van de skeletspieren. Ook andere chronische aandoeningen zoals hart-vaatziekten en symptomen van angst en depressie komen frequent voor bij patiënten met COPD. Volgens huidige schattingen heeft ongeveer $10 \%$ van de volwassen wereldbevolking een matig tot ernstige vorm van COPD. De verwachting is dat in 2030 deze aandoening de vierde meest voorkomende doodsoorzaak wereldwijd zal zijn.

\section{SPIERZWAKTE EN SPIERTRAINING}

Zwakte van de skeletspieren komt veel voor bij patiënten met COPD en draagt bij aan een afgenomen inspanningsvermogen, toegenomen gebruik van gezondheidszorgvoorzieningen en sterfte. Hoewel veel factoren bijdragen aan deze spierzwakte, speelt een gebrek aan lichamelijke activiteit van patiënten met COPD een belangrijke rol. Longrevalidatie is dan ook de meest succesvolle strategie om spierfunctie te verbeteren, symptomen te minimaliseren, zelfstandigheid te bevorderen en het functioneren tijdens dagelijkse activiteiten te laten toenemen bij patiënten met COPD. Patiënten met een hoge mate van kortademigheid zijn echter niet altijd in staat om hoog-intensieve duurtraining uit te voeren vanwege de hiermee gepaard gaande kortademigheid en vermoeidheid. Spierkrachttraining en transcutane neuromusculaire elektrostimulatie (NMES) kunnen effectieve alternatieve behandelmodaliteiten zijn om de spierfunctie te verbeteren. Er kan een onderscheid worden gemaakt tussen hoogfrequente (HF) en laagfrequente (LF) NMES. De focus van dit proefschrift is het onderzoeken van de effecten van NMES (onderverdeeld in HF-NMES en LF-NMES) en spierkrachttraining bij patiënten met COPD. 


\section{OVERZICHT VAN DIT PROEFSCHRIFT}

In hoofdstuk 2 werd de huidige literatuur systematisch bestudeerd aangaande effecten van NMES van de beenspieren bij patiënten met chronisch hartfalen (CHF) of COPD. 14 studies werden beoordeeld ( 9 studies onderzochten NMES bij patiënten met CHF patiënten en 5 bij patiënten met COPD). Veel studies rapporteerden significante verbeteringen in spierkracht, inspanningsvermogen en/of kwaliteit van leven. Deze systematische literatuurstudie liet zien dat NMES een geschikte revalidatie-interventie is bij patiënten met CHF of COPD.

Het doel van hoofdstuk 3 was om veranderingen in enzymactiviteit, veranderingen in spiervezeltypering en spiervezelgrootte in menselijke beenspieren na NMES systematisch te onderzoeken. 18 studies werden verzameld en in detail beoordeeld. Acht studies onderzochten veranderingen in enzymactiviteit, 7 studies onderzochten veranderingen in spiervezeltypering en 14 studies onderzochten veranderingen in spiervezelgrootte na NMES. De meeste LF-NMES studies rapporteerden significante veranderingen in oxidatieve enzymactiviteit, terwijl de resultaten betreffende veranderingen in spiervezeltypering en spiervezelgrootte conflicterend waren. De spiervezelgrootte nam in 55\% van de HF-NMES studies significant toe na. Echter, het was moeilijk om definitieve conclusies te trekken over de effecten van stimulatiefrequenties op veranderingen in de spieren vanwege het geringe aantal studies, de grote heterogeniteit in stimulatieparameters en verschillen in onderzoekspopulaties.

Omdat hoog-intensieve duurtraining vaak gelimiteerd is bij COPD patiënten vanwege een hoge mate van kortademigheid, was het doel van de hoofdstukken $\mathbf{4}$ en $\mathbf{5}$ om de metabole belasting (uitgedrukt in zuurstofconsumptie en ventilatie) en symptomen van kortademigheid en vermoeidheid te bestuderen gedurende spierkrachttraining en NMES. In hoofdstuk 4 werd de metabole belasting vergeleken gedurende één krachttrainingssessie met één sessie HF-NMES bij 13 patiënten met COPD. Beide trainingsmodaliteiten resulteerden in een acceptabel lage metabole belasting en symptomen. Hogere waardes werden gevonden tijdens spierkrachttraining dan tijdens HF-NMES. In hoofdstuk $\mathbf{5}$ waren de metabole belasting en symptomen vergelijkbaar tussen één sessie HF-NMES (75 Hertz) en één sessie LF-NMES (15 Hertz) bij 17 patiënten met COPD. HF-NMES, LF-NMES en spierkrachttraining lijken geschikte trainingsvormen om toe te passen bij ernstig kortademige patiënten met slecht functionerende beenspieren vanwege de lage metabole belasting en symptomen. Een prospectieve gerandomiseerde effectstudie (RCT) werd ontworpen om de effecten van HF-NMES, LF-NMES en spierkrachttraining te bestuderen bij COPD patiënten die gekenmerkt worden door een hoge mate van kortademigheid en zwakte van de beenspieren.

Hoofdstuk 6 gaf een overzicht van de klinische heterogeniteit, inclusief geslachtsverschillen, in een duidelijk beschreven subgroep van mensen met zeer ernstig 
COPD en een hoge mate van kortademigheid. Een grote spreiding werd gevonden voor diffusiecapaciteit, inspanningsvermogen, quadriceps spierfunctie en gezondheidstoestand bij 117 patiënten met COPD GOLD D. Zowel in problematische activiteiten van het dagelijks leven als in geobjectiveerde comorbiditeiten bestond eveneens een grote variatie. Bovendien werden significante geslachtsverschillen gevonden voor inspanningsvermogen, functioneren van de beenspieren en het vóórkomen van bijkomende aandoeningen.

Hoofdstuk 7 bestudeerde de effecten van HF-NMES (75 Hertz), LF-NMES (15 Hertz) of spierkrachttraining bij ernstig kortademige personen met COPD met spierzwakte van de bovenbenen bij aanvang van het onderzoek. 120 mensen met COPD werden door het lot toegewezen aan één van bovenstaande interventies. De kracht van de bovenbeenspieren nam toe na HF-NMES of spierkrachttraining, maar niet na LF-NMES. Het uithoudingsvermogen van de bovenbeenspieren, 6-minuten loopafstand, fietsduurvermogen, mate van kortademigheid, symptomen van depressie, problematische activiteiten van het dagelijks leven en de gezondheidstoestand verbeterden bij de personen in alle groepen vergeleken bij aanvang van het onderzoek met geen verschillen tussen de groepen.

In hoofdstuk 8 werd de metabole belasting tijdens de diverse vormen van lokale spiertraining 3 keer gemeten gedurende de studie; in de eerste week, vierde week en de achtste week van het revalidatieprogramma. De piek zuurstofopname en piek ventilatie bleven relatief laag gedurende HF-NMES, LF-NMES of spierkrachttraining met toenemende trainingsbelasting gedurende het revalidatieprogramma.

In hoofdstuk 9 werden de veranderingen in skeletspierkracht en veranderingen in inspanningsvermogen na HF-NMES, LF-NMES of spierkrachttraining bediscussieerd, inclusief de metabole belasting. Tevens werden toekomstige therapeutische mogelijkheden en wetenschappelijke vraagstellingen besproken.

\section{CONCLUSIES}

HF-NMES, LF-NMES of spierkrachttraining zijn toepasbare, veilige en effectieve trainingsvormen bij ernstig kortademige personen met COPD en spierzwakte verwezen voor longrevalidatie. Toename van de kracht en het uithoudingsvermogen van de bovenbeenspieren zijn hoger na HF-NMES in vergelijking met LF-NMES. HF-NMES is net zo effectief als spierkrachttraining bij COPD patiënten met een hoge mate van kortademigheid. Tot slot kan geconcludeerd worden dat de belasting van deze vormen van spiertraining voor het ademhalingssysteem van deze patiënten gering is. 

Dankwoord 
In dit laatste hoofdstuk wil ik graag alle mensen bedanken die hebben bijgedragen aan het proefschrift en me gesteund hebben tijdens het promotietraject, wat soms voelde als een hordenloop met de afstand van een marathon. Zonder deze mensen was de totstandkoming van dit proefschrift onmogelijk geweest.

Ten eerste een woord van dank aan alle patiënten met COPD die, ondanks hun hoge mate van kortademigheid en vele fysieke beperkingen, belangeloos hebben deelgenomen aan de verschillende onderzoeken. Zonder jullie medewerking en motivatie konden de beschreven studies in dit proefschrift niet uitgevoerd worden.

Mijn speciale dank gaat uit naar het promotieteam, bestaande uit mijn promotor Prof. Dr. Emiel Wouters en mijn copromotoren Dr. Martijn Spruit en Dr. Frits Franssen.

Prof. Wouters, ik wil u hartelijk danken voor de mogelijkheden die u mij de afgelopen jaren heeft geboden. In eerste instantie gaf $u$ mij de mogelijkheid om de masteropleiding Fysiotherapiewetenschap te volgen en vervolgens gaf u mij de mogelijkheid om me voltijds te kunnen richten op het promotieonderzoek. Ik heb veel bewondering voor uw expertise en toewijding ten aanzien van het COPD onderzoek waarmee uw wereldwijd bekend bent. Ondanks uw enorm drukke bestaan vond u tijd om me waardevol en kritisch te begeleiden.

Beste Martijn, ik ben je enorm dankbaar dat je mijn copromotor wilde zijn. Je was een geweldige begeleider, reeds voor ik gestart was met het promotietraject. Alle stukken die ik je stuurde werden zeer snel van kritisch en zinvol commentaar voorzien. Je bent een enorme inspiratiebron en ik heb veel respect voor jouw status binnen het internationale COPD onderzoek. Ik hoop nog lang met je te kunnen samenwerken!

Beste Frits, Ik ben jou ook ontzettend dankbaar voor alle opbouwende commentaren en begeleiding die je hebt gegeven. Je bent pas in een later stadium officieel toegevoegd aan het promotieteam, maar dit was een logische stap omdat je reeds een aantal jaren betrokken was bij mijn promotieonderzoek en coauteur was van verscheidene artikelen. Je bent een uitstekend voorbeeld hoe de je de klinische praktijk combineert met wetenschappelijk onderzoek.

Voor de kritische beoordeling en goedkeuring van het manuscript bedank ik de leden van de beoordelingscommissie, bestaande uit voorzitter Prof. Dr. H. Brunner-La Rocca, Prof. Dr. R. Gosselink, Prof. Dr. M. Hesselink, Prof. Dr. R. Smeets en Prof. Dr. M. Vanderthommen. 
Daarnaast wil ik Ingrid Augustin, lid van de Raad van Bestuur van CIRO, bedanken voor de geboden mogelijkheden en faciliteiten om mijn promotieonderzoek binnen CIRO uit te voeren en de kansen die ik heb gekregen om me verder te ontwikkelen.

Graag wil ik ook Paul Janssen bedanken. Ofschoon Paul niet direct betrokken is geweest bij de totstandkoming van het proefschrift, is hij tijdens mijn eerste jaren in CIRO een enorme stimulans en een bron van inspiratie geweest.

Natuurlijk wil ik de biometristen Martijn Cuijpers, Martyna Renckens en Jeannet Delbressine bedanken voor hun onmisbare assistentie. Hartelijk dank voor de metingen en de verzameling van de gegevens!

Fysiotherapeute en onderzoeker Anouk Vaes wil ik bedanken dat ze back-up wilde zijn tijdens de gerandomiseerde effectstudie naar de effecten van NMES. Anouk, ik wens je heel veel succes in de afronding van je eigen promotieonderzoek.

De fysiotherapeuten Sonja, Sigrid, Maud, Janneke, Chris, Leon en Maurice en de bewegingsagogen Jerôme, Tom, Chantal, Remco, Arjan, Silvia en Tatjana wil ik hartelijk bedanken voor hun ondersteuning van mijn onderzoek en de begeleiding van de patiënten.

De biometristen Marco en Koen bedank ik voor de instructie en hulp met de mobiele oxycon.

De leden van de onderzoeksgroep, Erica, Daisy, Lowie, Sarah, Dionne B, Miriam, Fiona, Carmen, Coby, Dionne S, Nienke, Wai-Yan, Riny, Annie, Vasilis en Rafael bedank ik voor de feedback, steun en gezelligheid tijdens de congressen.

Verder wil ik alle overige collega's van CIRO bedanken die betrokken zijn geweest bij de planning, diagnostiek en de revalidatie van de betrokken patiënten, voor de fijne samenwerking en de interesse in dit onderzoek.

Dank aan mijn paranimfen Ruud Beurskens en Roland Simons. Beste Ruud en Roland, ik geniet enorm tijdens het mountainbiken op de zondagochtend met jullie door de Swalmense bossen. Het is altijd een combinatie van fysieke inspanningen en gezellige gesprekken met veel humor. Ik hoop dat we dit nog lang zullen volhouden.

Kevin Derks wil ik bedanken voor alle tijd en energie in het ontwerpen van de cover van het proefschrift!

Bedankt aan Peter van Cruchten voor het kritisch nalezen van het proefschrift. 
Ik wil mijn ouders en schoonouders hartelijk bedanken voor de steun en interesse die ze in mijn werk en het onderzoek tonen. Daarnaast wil ik jullie bedanken dat jullie altijd klaarstaan voor mij en mijn gezin.

Tot slot wil ik natuurlijk Marit, Martijn en Marlou bedanken. Lieve Marit, de afgelopen jaren waren niet altijd even gemakkelijk vanwege de combinatie van het (promotie)onderzoek en twee opgroeiende kinderen. Ik wil je bedanken voor alle tijd en vrijheid die je me gunde om aan het onderzoek te besteden. Lieve Martijn en Marlou, jullie zijn fantastische kinderen en lieten mij geregeld vaktermen in lekentaal uitleggen. Het zit er op, de finish is bereikt! 

Curriculum vitae 


\section{CURRICULUM VITAE}

Maurice Sillen werd geboren op 29 november 1967 in Swalmen. In 1987 behaalde hij zijn HAVO diploma aan het Bisschoppelijk College Broekhin in Roermond en begon vervolgens aan de studie Fysiotherapie aan de Hogeschool in Heerlen. Na het behalen van zijn diploma heeft hij achtereenvolgens gewerkt in het Sint Maartens Gasthuis in Venlo, twee particuliere praktijken voor Fysiotherapie in Duitsland, Stichting Proteion en momenteel in CIRO. Van 2004 tot 2007 is hij lid geweest van de ondernemingsraad CIRO, waarvan het laatste jaar interim-voorzitter. Van 2006 tot 2009 heeft hij de masteropleiding Fysiotherapiewetenschap gevolgd aan de Universiteit in Utrecht. Aansluitend werd het promotieonderzoek gestart in CIRO Horn onder begeleiding van Prof. dr. E.F.M. Wouters, Dr. M.A. Spruit en Dr. F.M.E. Franssen. Daarnaast geeft hij sinds 2010 gastcolleges aan de hogescholen voor Fysiotherapie in Amsterdam, Heerlen en Enschede en hij is betrokken als extern adviseur bij afstudeerscripties aan de hogeschool in Eindhoven. Tevens is hij lid van de NPI expertgroep COPD voor fysiotherapeuten en lid van de revalidatiegroep van de European Respiratory Society. In 1994 is Maurice getrouwd met Marit en ze hebben twee kinderen, Martijn (1998) en Marlou (2001). Hobby's van Maurice zijn hardlopen en mountainbiken. 

List of publications 


\section{ORIGINAL RESEARCH ARTICLES AND REVIEWS}

Maurice J.H. Sillen, Frits M.E. Franssen, Jeannet M.L. Delbressine, Anouk W. Vaes, Emiel F.M. Wouters, Martijn A. Spruit. Metabolic load during strength training or NMES in individuals with COPD: results from the DICES trial. Submitted

Maurice J.H. Sillen, Frits M.E. Franssen, Jeannet M.L. Delbressine, Anouk W. Vaes, Emiel F.M. Wouters, Martijn A. Spruit. Efficacy of lower-limb muscle training modalities in severely dyspnoeic individuals with chronic obstructive pulmonary disease: results from the DICES trial. Thorax, 2014 in press

Maurice J.H. Sillen, Frits M.E. Franssen, Harry R. Gosker, Emiel F.M. Wouters, Martijn A. Spruit. Metabolic and structural changes in lower-limb skeletal muscle following neuromuscular electrical stimulation: a systematic review. PLoS One. 2013 Sept 8(9):e69391.

Martijn A. Spruit, Maurice J.H. Sillen, Miriam T. Groenen, Emiel F.M. Wouters, Frits M.E. Franssen. New normative values for handgrip strength: results from the UK Biobank. Journal of the American Medical Directors Association. 2013 Aug 16.

Maurice J.H. Sillen, Frits M.E. Franssen, Jeannet M.L. Delbressine, Nicole H.M.K. UszkoLencer, Lowie E.G.W. Vanfleteren, Erica P.A. Rutten, Emiel F.M. Wouters, Martijn A. Spruit. Heterogeneity in clinical characteristics and co-morbidities in dyspneic individuals with COPD GOLD D: Findings of the DICES trial. Respiratory Medicine. 2013 107(8):1186-94.

Maurice J.H. Sillen, Jan H. Vercoulen, Alex J. van 't Hul, Peter H.C. Klijn, Emiel F.M. Wouters, Dirk van Ranst, Jeannette B. Peters, Anton R.J. van Keimpema, Frits M.E. Franssen, Henk J.A.M. Otten, Johan Molema, Jerôme J. Jansen, Martijn A. Spruit. Inaccuracy of estimating peak work rate from six-minute walk distance in patients with COPD. COPD. 2012 Jun;9(3):281-8.

Marco A. Akkermans, Maurice J.H. Sillen, Emiel F.M. Wouters, Martijn A. Spruit. Validation of the oxycon mobile metabolic system in healthy subjects. Journal of Sports Science and Medicine. 2012 March; 11(1):182-3.

Maurice J. H. Sillen, Emiel F. M. Wouters, Frits M. E. Franssen, Kenneth Meijer, Koen H. P. Stakenborg, Martijn A. Spruit. Oxygen uptake, ventilation, and symptoms during lowfrequency versus high-frequency NMES in COPD: a pilot study. Lung. 2011 Feb;189(1):21-6. 
Maurice J. H. Sillen, Caroline M. Speksnijder, Rose-Miek A. Eterman, Paul P. Janssen, Scott S. Wagers, Emiel F. M. Wouters, Nicole H. M. K. Uszko-Lencer, Martijn A. Spruit. Effects of neuromuscular electrical stimulation of muscles of ambulation in patients with chronic heart failure or COPD: a systematic review of the English-language literature. Chest. 2009 Jul;136(1):44-61.

Maurice J. H. Sillen, Emiel F. M. Wouters, Frits M.E. Franssen, Martijn A. Spruit. Resistance training and neuromuscular electrical stimulation during acute exacerbations of chronic obstructive pulmonary disease. International Journal of Respiratory Care. 2009 May; 5(1): 14-6.

Maurice J.H. Sillen, Paul P. Janssen, Marco A. Akkermans, Emiel F.M. Wouters, Martijn A. Spruit. The metabolic response during resistance training and neuromuscular electrical stimulation (NMES) in patients with COPD, a pilot study. Respiratory Medicine. 2008 May;102(5):786-9.

\section{BOOK CHAPTER}

Maurice J.H. Sillen, Anouk W. Vaes, Frits M.E. Franssen, Emiel F.M. Wouters, Martijn A. Spruit. Trainingsstrategieën in revalidatieprogramma's bij patiënten met COPD. In Jaarboek Fysiotherapie Kinesitherapie. 2011:123-33. 

